\title{
Dephasing and Phase-Coherence in disordered mesoscopic Conductors
}

\author{
Dissertation \\ zur Erlangung des Doktorgrades der \\ Mathematisch-Naturwissenschaftlichen Fakultäten \\ der Georg-August-Universität zu Göttingen
}

vorgelegt von

Axel Völker

aus Georgsmarienhütte

Göttingen 2000 
D 7

Referent: Prof. Dr. P. Kopietz

Korreferent: Prof. Dr. R. Kree

Tag der mündlichen Prüfung: 27. April 2000 


\section{Danksagung}

Ich danke Peter Kopietz für die Anregung zu dieser Arbeit sowie deren sehr engagierte Betreuung. Die enge Zusammenarbeit mit ihm sowie seine unkomplizierte und motivierende Art haben für ein stets hervorragendes Arbeitsklima gesorgt. Besonders bedanken möchte ich mich für die Ermöglichung der Gastaufenthaltes an der University of California Los Angeles. Herrn Prof. Dr. K. Schönhammer danke ich für Diskussionen und Anregungen, sowie Herrn Prof. Dr. R. Kree für die Übernahme des Korreferats. Jürgen Holm danke ich für geduldige Hilfe bei allen Problemen mit der Rechenanlage und seine hervorragende "Master-Slave Implementation für PVM". Finanziell wurde diese Arbeit durch den Sonderforschungsbereich 345 'Festkörper weit weg vom Gleichgewicht' unterstützt.

In besonders angenehmer Erinnerung wird mir die gemeinsame Büro- und Freizeit mit Christian F. Wöhler bleiben. Zur guten Stimmung am Institut haben auch Sören Addicks, Nias Donlagic, Tom Busche, Lorenz Bartosch, Tobias Stauber, Thomas Östreich und Volker Meden entscheidend beigetragen. Letzteren beiden danke ich zudem für viele lehrreiche fachliche Diskussionen. Meinen Freunden in Osnabrück und Göttingen danke ich dafür, daß sie durch die gemeinsame Zeit für den nötigen Ausgleich zur Physik und somit für neue Motivation gesorgt haben. Besondere Dank gilt meinen Eltern Hans und Almut Völker ohne deren Vertrauen und Unterstützung dies Arbeit unmöglich gewesen wäre. 


\section{Contents}

1 Introduction $\quad 6$

2 Dephasing in disordered conductors 8

2.1 Basic concepts and definitions . . . . . . . . . . . . 8

2.2 Connection between dephasing and weak localization . . . . . . . . . 10

2.3 Weak localization in the presence of external dephasing fields . . . . . 18

2.4 The dephasing time to first order in the screened interaction . . . . . 27

3 Eikonal approach to dephasing in disordered conductors 36

3.1 lntroduction . . . . . . . . . . . . . . . . . . . . . 36

3.2 The Cooperon in a fluctuating scalar potential: Eikonal expansion . . 37

3.3 Dephasing due to Nyquist noise in arbitrary dimensions . . . . . . . . . 40

3.3.1 The Debye-Waller factor . . . . . . . . . . . . . . . . . . . . 44

3.3.2 The dephasing rate in infinite systems . . . . . . . . . . . . . 46

3.3.3 The dephasing rate in finite systems . . . . . . . . . . . . . 53

3.3.4 How first order perturbation theory is reproduced from the Eikonal approximation . . . . . . . . . . . . . 55

3.4 Dephasing due to external electric fields $\ldots \ldots \ldots$. . . . . . . . 57

3.4.1 Uniform electric field . . . . . . . . . . . . . . . . . . 59

3.4.2 Dephasing due to longitudinal electric fields with finite wave-

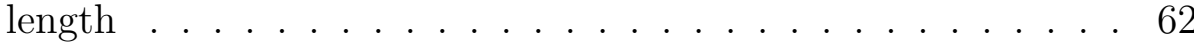

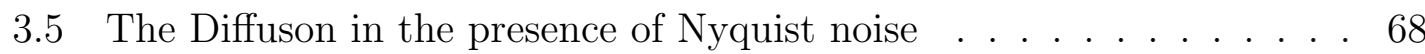

3.5.1 Infinite systems . . . . . . . . . . . . . . . . . . 71

3.5.2 Finite systems . . . . . . . . . . . . . . . . . . . 75 
$\begin{array}{lll}4 & \text { Non-linear response in mesoscopic metal rings } & 77\end{array}$

4.1 Introduction . . . . . . . . . . . . . . . . . . 77

4.2 The quadratic response function: What is wrong with the Green's function approach? . . . . . . . . . . . . . . . . 80

4.3 Adiabatic switching on . . . . . . . . . . . . . . . . . 85

4.3 .1 Comparison with sudden switching on . . . . . . . . 87

4.4 Numerical results . . . . . . . . . . . . . . . . . . . . . . . . . 89

4.4 .1 The static limit of the response function . . . . . . . . . . . . 94

4.4 .2 The response function for finite frequencies $\varepsilon>0$. . . . . . . 98

4.4 .3 Influence of the boundary conditions in transverse directions . 102

4.4 .4 The linear in time contribution to the current . . . . . . . . . 105

4.5 Conclusions . . . . . . . . . . . . . . . . . 107

5 Persistent currents and electron-electron interactions 110

5.1 Introduction . . . . . . . . . . . . . . . . . . . . . . . . . 110

$5.2 \quad$ First order RPA corrections to the persistent current . . . . . . . . . 112

5.3 The long wave-length contribution to the Fock current . . . . . . . . 115

$\begin{array}{lll}6 & \text { Summary } & 121\end{array}$

A How higher order response functions can be calculated within the $\begin{array}{ll}\text { Matsubara technique } & 123\end{array}$

A.1 The real time response function . . . . . . . . . . . . . . . . . . . . 123

A.2 The Matsubara response function . . . . . . . . . . . . . . . . . 127

\begin{tabular}{ll}
\hline B Fluctuation-dissipation theorem & 131
\end{tabular} 


\section{Chapter 1}

\section{Introduction}

Due to the technical progress in low temperature and nano-scale physics there is by now a large number of experiments which clearly reveal quantum mechanical interference effects in the electronic properties of metals and semiconductors. Weak localization, persistent currents, and universal conductance fluctuations are well known examples where the quantum nature of the electrons becomes important for their dynamics even in samples that are many orders of magnitude lager than atomic scales. At higher temperatures such systems can usually be described by the semiclassical Drude-Sommerfeld picture which only treats the electrons statistics quantum mechanical but assumes a classical motion. Since at lower temperatures the above mentioned effects occur as corrections to the classical results, there must be some temperature dependent parameter that measures the importance of interference effects in solid state physics. For this purpose the phase-coherence length $L_{\varphi}$ is introduced. In a phenomenological picture $L_{\varphi}$ is interpreted as the distance an electron typically moves, before all information about its initial phase is lost. Closely related to $L_{\varphi}$ is the phase coherence time $\tau_{\varphi}$ which is just the average time an electron needs to travel the distance $L_{\varphi}$. The length scale $L_{\varphi}$ defines a new class of physical systems, so called mesoscopic systems. 'Mesoscopic' denotes an intermediate size which is much lager than atomic dimensions but smaller than 'macroscopic'. Such a sample is large enough to be described by the statistical concepts of condensed matter physics, but its typical extension $L$ is smaller than $L_{\varphi}$. Electrons can propagate coherently through the entire probe and thus become sensitive to the geometry of the sample and the precise distribution of impurities. E.g. the persistent current in mesoscopic metal rings manifests the strong dependence on boundary conditions while universal conductance fluctuations demonstrate the sensitivity to the disorder configuration. Whether a system is mesoscopic or not strongly depends, like $L_{\varphi}$ itself, on the temperature. In metals one experimentally finds phase coherence lengths of up to $L_{\varphi} \sim 10 \mu \mathrm{m}$ at temperatures $T \approx 10 \mathrm{mK}$. Due to the efforts to continuously decrease the size of semiconductor devices in computer technology, mesoscopic physics becomes of increasing practical importance. 
This work is organized as follows: Chapter 2 gives an introduction to the basic concepts of dephasing in disordered metallic systems. The close connection between $\tau_{\varphi}$ and the weak localization correction to the conductivity is outlined and the theoretical tools to derive the dephasing time are provided. To emphasize the difficulties which arise in a perturbative calculation of $\tau_{\varphi}$, some well known results of first order perturbation theory in the screened electron-electron interaction are rederived.

In chapter 3 a novel approach to calculate the dephasing time in disordered conductors is presented. The applied method has, compared to earlier calculations, the advantage that it does not require to introduce any cutoffs and is thus free of ambiguities. It is applied to study the influence of electron-electron interactions in a semiclassical limit as well as dephasing due to external fields. For the first case a single formula is given that allows to calculate the frequency as well as the temperature dependence of $\tau_{\varphi}$ for macroscopic and mesoscopic systems in arbitrary dimensions. Regarding external fields, the influence of a finite wave-length $\mathbf{q}_{0}$ is investigated and a new effect is found. The finding is in agreement with so far unexplained experimental data. Last but not least the Diffuson propagator is examined in the presence of dephasing fields. Chapter 3 is an extension of the work published in Ref. [1] and includes details of the calculations as well as previously unpublished results.

The first part of chapter 4 is a detailed version of Ref. [2] while in the second part new numerical results are presented. This chapter is devoted to non-equilibrium effects in mesoscopic metal rings. The response to a time dependent external field is explicitly studied and consequences of the discrete energy spectrum in finite systems are discussed. It is shown, that the standard Green's function approach can not be applied in this case. All findings were extensively tested numerically for a tightbinding model. Also the influence of the underlying statistical ensemble (canonical or grand canonical), which is known to be important in equilibrium, is investigated.

Since there is a close relation between non-linear response and electron-electron interactions, the latter are reconsidered in chapter 5. The experimentally observed persistent current is still not satisfactory understood. A common believe is, that the Coulomb interaction of the electrons is responsible for the discrepancy between theory and experiment. Theoretical attempts to include this effects to first order in the screened interaction could yet not solve this problem. Furthermore there was still one regime which had not been investigated previously since it is difficult to access within the standard diagrammatic approach. This is the long wave-length Fock contribution to the current. A simple way to circumvent the apparent difficulties is presented and this gap can be closed. In compact form the findings of chapter 5 have been published in Ref. [3]. 


\section{Chapter 2}

\section{Dephasing in disordered conductors}

\subsection{Basic concepts and definitions}

There are by now many review articles and books giving extensive introductions to the fundamental concept of phase-coherence and dephasing in many-particle systems [4, 5, 7, 6]. Therefore here only a brief summary will be given. Throughout this work the Planck constant $\hbar$ and the Boltzmann constant $k_{B}$ are set equal to unity; $\hbar=1$, $k_{B}=1$. In the following, the dimension $d$ will always be the effective dimension regarding diffusion. Two cases must be distinguished: True $d$-dimensional samples characterized by the $d$-dimensional density of states $\nu_{d}$ and a volume $\mathcal{V}=L^{d}$, and quasi $d$-dimensional systems, where the density of states is still the three dimensional one. In the latter case the volume is assumed to be of the form $\mathcal{V}=L^{d} a^{3-d}$ with $L$ much longer than the mean free path $l$, while $a$ is of the order of $l$. Diffusion can only take place on distances much longer than $l$ and thus the probe is $d$-dimensional from the diffusive point of view. Unless explicitly stated differently, all following calculations are valid for true as well as for quasi $d$-dimensional systems, if in the latter case the convention $\nu_{d}=\nu_{3} a^{3-d}$ is used. The average level spacing at the Fermi energy is connected to the $d$-dimensional density of states via $\Delta=1 /\left(\nu_{d} L^{d}\right)$.

The phase-coherence length is introduced in a quasiparticle picture and describes the average distance an electron travels before all information about its initial phase is lost [5, 8, 9]. A pedagogical review about dephasing and quantum transport in disordered systems has recently been given by Zwerger [4]. The investigated systems are disordered metals where $l$ is much longer than the Fermi wave-length. Expressed through the Fermi momentum $k_{F}$ this means $k_{F} l \gg 1$. Such samples are usually well described by the semiclassical Drude-Sommerfeld model [4, 10] where it is assumed 
that the electrons move classically between instantaneous collisions. Following Zwerger the probability amplitude for an electron to propagate from a point $\mathbf{r}$ to $\mathbf{r}^{\prime}$ at fixed energy $\varepsilon$ is in the semiclassical limit given by

$$
K\left(\mathbf{r} \rightarrow \mathbf{r}^{\prime}, \varepsilon\right) \approx \sum_{\alpha} A_{\alpha} e^{i S_{\alpha}}
$$

The sum extends over all classical paths $\alpha$ with the corresponding classical actions $S_{\alpha}$. Gaussian fluctuations around the trajectories $\alpha$ are integrated out, leading to the prefactors $A_{\alpha}$. The probability for an electron to propagate from $\mathbf{r}$ to $\mathbf{r}^{\prime}$ is given by the absolute square of $K$. Thus the probability for a particle to return to its starting point is

$$
|K(\mathbf{r} \rightarrow \mathbf{r}, \varepsilon)|^{2} \approx \sum_{\alpha, \beta} A_{\alpha} A_{\beta}^{*} e^{i S_{\alpha}-i S_{\beta}}
$$

and the sum now runs over all closed trajectories. Obviously for $\alpha=\beta$ the phase factors cancel. The system is said to be invariant under 'time-reversal', if a sudden reversion of the electrons momentum would cause the particle to traverses backwards along the trajectory it came from. E.g. in the presence of an external magnetic field this is not the case. Now if time-reversal symmetry is given, there is another contribution with zero phase-difference, namely if the trajectory $\beta$ is the time-reversed of $\alpha$, i.e. $\beta$ is just $\alpha$ traversed in opposite direction. This will be denoted by $\beta=\tilde{\alpha}$. Since the phase-difference between two different paths $\alpha$ and $\beta$ will generally be large, the sum over different trajectories will average out. Only the two classes of trajectories with equal phases, i.e. $\alpha=\beta$ and $\beta=\tilde{\alpha}$ will yield non-vanishing contributions after summing over all possible classical paths. In a purely classical description the term with $\beta=\tilde{\alpha}$ would be absent and thus the quantum mechanical return probability is twice the classical probability. In any realistic many particle system there will be processes which destroy the time-reversal invariance. Inelastic electron-electron or electron-phonon scattering and interactions with external electromagnetic fields are the most important examples. These effects suppress the coherence between closed trajectories $\alpha$ and their time-reversed counterpart $\tilde{\alpha}$. This suppression will increase with increasing circumference $L_{\alpha}$ of $\alpha$. At a certain value of $L_{\alpha}$, there will be no more interference between $\alpha$ and $\tilde{\alpha}$ and this value is called the phase-coherence length $L_{\varphi}$ of the system. One may alternatively think of a ring-geometry and define $L_{\varphi}$ as the maximum circumference of the ring, for which quantum effects due to the coherent propagation of the electrons around the ring can be observed. The phase coherence time is correspondingly defined as the typical time the particle needs to travel the distance $L_{\varphi}$. In strongly disordered systems the particles motion is diffusive and $\tau_{\varphi}$ is connected to $L_{\varphi}$ via

$$
\frac{1}{\tau_{\varphi}}=\frac{D}{L_{\varphi}^{2}}
$$


Here $D=v_{F}^{2} \tau_{e l} / d$ is the classical diffusion coefficient in $d$ dimensions for systems with Fermi-velocity $v_{F}$ and elastic mean free time of flight $\tau_{e l}$. From the fact that also elastic interactions, e.g. with magnetic fields, can affect the phase coherence time it is clear, that $1 / \tau_{\varphi}$ is not the same as the inelastic quasiparticle level-broadening $\gamma_{q p}$. At low temperatures $\gamma_{q p}$ is governed by the inelastic electron-electron scattering rate $1 / \tau_{e e}$. If the elastic and inelastic mechanisms are independent, $1 / \tau_{\varphi}$ is just the sum of both relaxation rates, $1 / \tau_{\varphi}=\gamma_{q p}+\gamma_{d e p h}$, where $\gamma_{d e p h}$ contains all elastic dephasing contributions.

The phase coherence length allows a new distinction of solid state samples. In macroscopic systems $L_{\varphi}$ is much smaller than the extension of the probe in any direction. Such systems are insensitive to changes in the boundary conditions. In contrast, so called mesoscopic systems are characterized by the condition that their typical extension $L$ is smaller than $L_{\varphi}$. In such probes coherence effects on the electronic transport in metals become extremely important. The probably most natural systems for studying such quantum effects are thin, quasi one-dimensional rings with circumference $L<L_{\varphi}$ which are pierced by a Aharonov-Bohm flux $\Phi$. By now there are lots of experiments and even more theoretical works dealing with such samples. Nevertheless, most of their fundamental features, like e.g. persistent currents, are yet not fully understood. In mesoscopic systems another important energy scale is introduced, the so called Thouless energy $E_{c}=D / L^{2}$. In units of $\hbar=1$ this is the energy scale associated with the average time a particle needs to diffuse through the sample of length $L$. The Thouless energy thus gives a measure for the sensitivity to changes in the boundary conditions of the system. In the Aharonov-Bohm ring geometry such changes can be realized through the external flux $\Phi$. The condition $L_{\varphi}>L$ for the system to be mesoscopic can now be written as $1 / \tau_{\varphi}<E_{c}$. Together with the average level-spacing at the Fermi energy, $\Delta$, there are thus three energy scales which define a finite system: $1 / \tau_{\varphi}, E_{c}$ and $\Delta$. In a diffusive metallic system their values must be much smaller than the elastic momentum relaxation rate $1 / \tau_{e l}$ which itself must satisfy $1 / \tau_{e l} \ll \varepsilon_{F}$, where $\varepsilon_{F}$ is the Fermi energy. Two different physical situations have to be distinguished when dealing with mesoscopic systems: First the 'continuous' spectrum case with $1 / \tau_{\varphi}>\Delta$, where the dephasing rate exceeds the average level spacing, and second the 'discrete' spectrum limit with $1 / \tau_{\varphi}<\Delta$. It will turn out, that the behavior of the probe can be completely different in either case.

\subsection{Connection between dephasing and weak lo- calization}

The phase coherence time was introduced in the previous section using a singleor, more generally, quasiparticle picture, which is useful to give a physical intuition 
for the quantities $\tau_{\varphi}$ and $L_{\varphi}$. In an interacting many-particle system it is however difficult to think of the phase of a single electron as a meaningful object and definitely such a thing can not be measured directly. Therefore one has to look for an observable quantity, which can directly be related to $\tau_{\varphi}$ in the quasiparticle picture. Such an observable can be found in the theory of weak localization. It was already mentioned that without any dephasing the quantum mechanical return probability is twice as large as the classical one, due to "coherent backscattering". This leads to an enhancement of the resistivity above the classical Drude result and is known as "weak localization". Now all processes that break the time-reversal invariance and therefore lead to a finite dephasing time tend to suppress the return probability and decreases the resistivity towards its classical value. By measuring the weak localization correction to the conductivity one can hence gain information about $\tau_{\varphi}$.

To be more specific, consider a system of non-interacting electrons in an transversal external electric driving-field $\mathbf{E}_{d r i}$ which is derived from a vector-potential $\mathbf{A}_{d r i}$ as

$$
\mathbf{E}_{d r i}(\mathbf{r}, t)=-\frac{1}{c} \frac{\partial \mathbf{A}_{d r i}(\mathbf{r}, t)}{\partial t}
$$

The corresponding single particle Hamiltonian is

$$
\hat{H}(t)=\sum_{j}\left\{\frac{1}{2 m}\left[\hat{\mathbf{P}}_{j}+\frac{e}{c} \mathbf{A}_{d r i}\left(\hat{\mathbf{R}}_{j}, t\right)\right]^{2}+U\left(\hat{\mathbf{R}}_{j}\right)\right\} .
$$

Here $\hat{\mathbf{P}}_{j}=-i \nabla_{\mathbf{R}_{j}}$ and $\hat{\mathbf{R}}_{j}$ are the canonical momentum operator and the position operator of the $j$-th particle. The sum in Eq.(2.5) extends over all electrons in the system. $U\left(\hat{\mathbf{R}}_{j}\right)$ is the static disorder potential, $-e$ the electron charge, $m$ its mass and $c$ the velocity of light. The standard way to treat disorder is to assume a delta-correlated distribution with zero mean. Denoting averaging over disorder by an over-bar, this means

$$
\overline{U(\mathbf{r})}=0 \quad ; \quad \overline{U(\mathbf{r}) U\left(\mathbf{r}^{\prime}\right)}=\tilde{\gamma} \mathcal{V} \delta\left(\mathbf{r}-\mathbf{r}^{\prime}\right) .
$$

$\mathcal{V}$ is the volume of the system and the factor $\tilde{\gamma}$ can in first order Born-approximation be related to the average level-spacing at the Fermi energy and the elastic mean free time of flight [11,28]:

$$
\tilde{\gamma}=\frac{\Delta}{2 \pi \tau_{e l}}
$$

The Hamiltonian can be rewritten as the sum of a time-independent contribution $\hat{H}_{0}$ and a time-dependent perturbation $\hat{H}_{1}(t)$, i.e. $\hat{H}(t)=\hat{H}_{0}+\hat{H}_{1}(t)$ with

$$
\hat{H}_{0}=\sum_{j}\left\{\frac{\hat{\mathbf{P}}_{j}^{2}}{2 m}+U\left(\hat{\mathbf{R}}_{j}\right)\right\},
$$




$$
\hat{H}_{1}(t)=\frac{e}{2 m c} \sum_{j}\left[\hat{\mathbf{P}}_{j} \mathbf{A}_{d r i}\left(\hat{\mathbf{R}}_{j}, t\right)+\mathbf{A}_{d r i}\left(\hat{\mathbf{R}}_{j}, t\right) \hat{\mathbf{P}}_{j}\right]+\frac{e^{2}}{2 m c^{2}} \sum_{j} \mathbf{A}_{d r i}^{2}\left(\hat{\mathbf{R}}_{j}, t\right) .
$$

In the following it will be more convenient to work in second quantization. The creation- and annihilation operators for a particle in momentum-state $\mathbf{q}$ are denoted by $\hat{\Psi}_{\mathbf{q}}^{\dagger}$ and $\hat{\Psi}_{\mathbf{q}}$. Correspondingly $\hat{\Psi}^{\dagger}(\mathbf{r})=\frac{1}{\sqrt{\mathcal{V}}} \sum_{\mathbf{q}} e^{-i \mathbf{q} \cdot \mathbf{x}} \hat{\Psi}_{\mathbf{q}}^{\dagger}$ and $\hat{\Psi}(\mathbf{r})=\frac{1}{\sqrt{\mathcal{V}}} \sum_{\mathbf{q}} e^{i \mathbf{q} \cdot \mathbf{x}} \hat{\Psi}_{\mathbf{q}}$ create or annihilate electrons in position-state $\mathbf{r}$. In this representation the above equations read

$$
\hat{H}_{0}=\sum_{\mathbf{q}} \frac{\mathbf{q}^{2}}{2 m} \hat{\Psi}_{\mathbf{q}}^{\dagger} \hat{\Psi}_{\mathbf{q}}+\int d \mathbf{r} U(\mathbf{r}) \hat{\Psi}^{\dagger}(\mathbf{r}) \hat{\Psi}(\mathbf{r})
$$

and

$$
\hat{H}_{1}(t)=-\frac{1}{c} \int d \mathbf{r} \mathbf{A}_{d r i}(\mathbf{r}, t) \hat{\mathbf{j}}_{p a r a}(\mathbf{x})+\frac{e^{2}}{2 m c^{2}} \int d \mathbf{r} \mathbf{A}_{d r i}^{2}(\mathbf{r}, t) \hat{\Psi}^{\dagger}(\mathbf{r}) \hat{\Psi}(\mathbf{r}) .
$$

In linear order the vector-potential $\mathbf{A}_{d r i}$ couples to the paramagnetic current operator

$$
\hat{\mathbf{j}}_{\text {para }}(\mathbf{r})=\frac{(-e)}{2 m}\left[-i \hat{\Psi}^{\dagger}(\mathbf{r}) \nabla \hat{\Psi}(\mathbf{r})+\left(i \nabla \hat{\Psi}^{\dagger}(\mathbf{r})\right) \hat{\Psi}(\mathbf{r})\right] .
$$

The total current operator is the sum of the paramagnetic and the diamagnetic part, $\hat{\mathbf{j}}(\mathbf{r}, t)=\hat{\mathbf{j}}_{\text {para }}(\mathbf{r})+\hat{\mathbf{j}}_{\text {dia }}(\mathbf{r}, t)$, with

$$
\hat{\mathbf{j}}_{d i a}(\mathbf{r}, t)=-\frac{e^{2}}{m c} \mathbf{A}_{d r i}(\mathbf{r}, t) \hat{\Psi}^{\dagger}(\mathbf{r}) \hat{\Psi}(\mathbf{r}) .
$$

All the above operators are in the Schrödinger representation. The current $\mathbf{j}(\mathbf{r}, t)=$ $\left\langle\hat{\mathbf{j}}_{H}(\mathbf{r}, t)\right\rangle$ is just the expectation-value $\langle\cdots\rangle=\left(1 / Z_{0}\right) \operatorname{Sp}\left[\exp \left(-\beta \hat{H}_{0}\right) \cdots\right]$ of the current-operator in the Heisenberg representation (indicated by $H$ ) over the initial thermal state of the system. Here the definitions $\beta:=1 / T$ and $Z_{0}=\operatorname{Sp}\left[\exp \left(-\beta \hat{H}_{0}\right)\right]$ were introduced. For explicit calculations it is often more convenient to work in the momentum-frequency representation. From Eqs.(2.11) and (2.12) one gets

$$
\begin{gathered}
\hat{H}_{1}(t)=\frac{e}{2 m c \mathcal{V}} \sum_{\mathbf{q}, \mathbf{q}^{\prime}} \mathbf{A}_{d r i}\left(\mathbf{q}-\mathbf{q}^{\prime}, t\right) \cdot\left[\mathbf{q}+\mathbf{q}^{\prime}\right] \hat{\Psi}_{\mathbf{q}}^{\dagger} \hat{\Psi}_{\mathbf{q}^{\prime}}+\mathcal{O}\left(A_{d r i}^{2}\right), \\
\mathbf{j}_{\text {para }}(\mathbf{Q}, t)=-\frac{e}{2 m} \sum_{\mathbf{k}}(\mathbf{Q}+2 \mathbf{k})\left\langle\hat{\Psi}_{\mathbf{Q}+\mathbf{k}}^{\dagger} \hat{\Psi}_{\mathbf{k}}\right\rangle .
\end{gathered}
$$

$\mathbf{A}_{d r i}(\mathbf{q}, \omega)=\int d \mathbf{r} d t e^{-i(\mathbf{q} \cdot \mathbf{r}-\omega t)} \mathbf{A}_{d r i}(\mathbf{r}, t)$ and $\mathbf{j}(\mathbf{Q}, \omega)=\int d \mathbf{r} d t e^{-i(\mathbf{Q} \cdot \mathbf{r}-\omega t)} \mathbf{j}(\mathbf{r}, t)$ are the Fourier transforms of the vector potential and current density. The conductivity tensor is defined as

$$
j_{i}(\mathbf{r}, t)=\sum_{j} \int d \mathbf{r}^{\prime} d t^{\prime} \sigma_{i, j}\left(\mathbf{r}, \mathbf{r}^{\prime}, t-t^{\prime}\right) E_{d r i, j}\left(\mathbf{r}^{\prime}, t^{\prime}\right) .
$$


Here $i$ and $j$ denote the Cartesian components of the vectors. It is assumed, that after averaging over disorder the system is spatially homogeneous and isotropic so that the conductivity tensor is symmetric and of the form $\bar{\sigma}_{i, j}\left(\mathbf{r}, \mathbf{r}^{\prime}, t-t^{\prime}\right)=$ $\delta_{i, j} \bar{\sigma}\left(\mathbf{r}-\mathbf{r}^{\prime}, t-t^{\prime}\right)$. Since Eq.(2.16) now becomes a convolution, it is convenient to go over to the wave-vector and frequency representation:

$$
\mathbf{j}(\mathbf{Q}, \omega)=\bar{\sigma}(\mathbf{Q}, \omega) \mathbf{E}_{d r i}(\mathbf{Q}, \omega),
$$

with $\mathbf{E}_{d r i}(\mathbf{Q}, \omega)=\frac{i \omega}{c} \mathbf{A}_{d r i}(\mathbf{Q}, \omega)$. To obtain the weak localization correction to the conductivity for the system described by the Hamiltonian (2.5) the current $\mathbf{j}$ has to be calculated to linear order in the external field $\mathbf{E}_{d r i}$. This is most conveniently done by using either the Keldysh or the Matsubara technique (see Appendix A). Here only a spatially constant field with $\mathbf{Q}=0$ will be considered and to simplify the notation this variable is suppressed in the following, $\mathbf{j}(\omega):=\mathbf{j}(\mathbf{Q}=0, \omega)$. (Note that $\mathbf{j}(\omega)$ is $\mathcal{V} \times$ the spatially averaged current density.) Using the notation of App.A, the time dependent perturbation is to first order in $\mathbf{A}_{d r i}$ given by

$$
V_{\mathbf{q}, \mathbf{q}^{\prime}}(\omega)=\frac{e}{2 m c \mathcal{V}} \mathbf{A}_{d r i}\left(\mathbf{q}-\mathbf{q}^{\prime}, \omega\right) \cdot\left[\mathbf{q}+\mathbf{q}^{\prime}\right]
$$

(Compare with Eq.(2.14)). From the first order contribution

$$
\mathbf{j}_{\text {para }}(\omega)=-\frac{e}{m} \sum_{\mathbf{k}} \mathbf{k} g^{(1)}(\omega)
$$

with $g^{(1)}(\omega)$ given in Eq. (A.15) one obtains

$$
\begin{gathered}
\mathbf{j}_{\text {para }}(\omega)=-\frac{e^{2}}{2 m^{2} \mathcal{V}_{c}} \sum_{\mathbf{k}} \mathbf{k} \frac{1}{2 i} \sum_{\mathbf{q}, \mathbf{q}^{\prime}}\left(\mathbf{A}_{d r i}\left(\mathbf{q}^{\prime}-\mathbf{q}, \omega\right) \cdot\left[\mathbf{q}+\mathbf{q}^{\prime}\right]\right) \times \\
\int_{-\infty}^{\infty} \frac{d \varepsilon}{2 \pi} \operatorname{Sp}\left[\underline{\sigma}_{1} \underline{G}_{\mathbf{k}, \mathbf{q}}^{0}(\varepsilon+\omega) \underline{G}_{\mathbf{q}^{\prime}, \mathbf{k}}^{0}(\varepsilon)\right] .
\end{gathered}
$$

To obtain the average conductivity Eq. $(2.20)$ has to be averaged over disorder. This procedure restores translational invariance, so that after averaging only terms with $\mathbf{q}=\mathbf{q}^{\prime}$ survive. Expressing the vector potential through the electric field yields the following equation for the paramagnetic part of the conductivity tensor:

$$
\left(\bar{\sigma}_{\text {para }}\right)_{i, j}(\omega)=\frac{e^{2}}{2 \omega m^{2} \mathcal{V}} \sum_{\mathbf{k}, \mathbf{q}} k_{i} q_{j} \int_{-\infty}^{\infty} \frac{d \varepsilon}{2 \pi} \operatorname{Sp}\left[\underline{\sigma}_{1} \overline{G_{\mathbf{k}, \mathbf{q}}^{0}(\varepsilon+\omega) \underline{G}_{\mathbf{q}, \mathbf{k}}^{0}(\varepsilon)}\right] .
$$

Using $\bar{\sigma}_{i, j}(\omega)=\delta_{i, j} \bar{\sigma}(\omega)$ one can express $\bar{\sigma}(\omega)$ as $\bar{\sigma}(\omega)=1 / d \sum_{i=1}^{d} \bar{\sigma}_{i i}$ and thus

$$
\bar{\sigma}_{\text {para }}(\omega)=\frac{e^{2}}{2 \omega d m^{2} \mathcal{V}} \sum_{\mathbf{k}, \mathbf{q}} \mathbf{k} \cdot \mathbf{q} \int_{-\infty}^{\infty} \frac{d \varepsilon}{2 \pi} \operatorname{Sp}\left[\underline{\sigma}_{1} \overline{G_{\mathbf{k}, \mathbf{q}}^{0}(\varepsilon+\omega) \underline{G}_{\mathbf{q}, \mathbf{k}}^{0}(\varepsilon)}\right] .
$$


Now evaluating the trace making use of Eq.(A.10) and adding the diamagnetic contribution finally yields

$$
\begin{array}{r}
\bar{\sigma}(\omega)=\frac{e^{2}}{2 \omega d m^{2} \mathcal{V}} \sum_{\mathbf{k}, \mathbf{q}} \mathbf{k} \cdot \mathbf{q} \int_{-\infty}^{\infty} \frac{d \varepsilon}{2 \pi}\left\{h(\varepsilon) \overline{\mathcal{G}_{\mathbf{k}, \mathbf{q}}^{0, R}(\omega+\varepsilon)\left[\mathcal{G}_{\mathbf{q}, \mathbf{k}}^{0, R}(\varepsilon)-\mathcal{G}_{\mathbf{q}, \mathbf{k}}^{0, A}(\varepsilon)\right]}\right. \\
+h(\omega+\varepsilon) \overline{\left.\left[\mathcal{G}_{\mathbf{k}, \mathbf{q}}^{0, R}(\omega+\varepsilon)-\mathcal{G}_{\mathbf{k}, \mathbf{q}}^{0, A}(\omega+\varepsilon)\right] \mathcal{G}_{\mathbf{q}, \mathbf{k}}^{0, A}(\varepsilon)\right\}}-\frac{e^{2} n}{i \omega m}
\end{array}
$$

Here $n=\overline{\left\langle\hat{\Psi}^{\dagger}(\mathbf{r}) \hat{\Psi}(\mathbf{r})\right\rangle}=N / \mathcal{V}$ is the average particle density. The notation is chosen as in Appendix A, i.e. $h(\varepsilon)=\tanh (\varepsilon \beta / 2)$. Disorder averaging is performed using standard diagrammatic perturbation theory [11, 12]. The diamagnetic part which diverges as $1 / \omega$ is canceled by the contribution from the $\mathcal{G}^{0, A} \mathcal{G}^{0, A}$ and $\mathcal{G}^{0, R} \mathcal{G}^{0, R}$ terms [12]. Calculating the single-particle Green's function in Born approximation yields [11]

$$
\overline{\mathcal{G}_{\mathbf{k}, \mathbf{p}}^{0, R / A}(\omega)}=: \delta_{\mathbf{k}, \mathbf{p}}{\overline{\mathcal{G}^{0}}}_{\mathbf{k}}^{R / A}(\omega)=\delta_{\mathbf{k}, \mathbf{p}} \frac{1}{\omega-\xi_{k} \pm \frac{i}{2 \tau_{e l}}},
$$

where $\xi_{k}=\mathbf{k}^{2} / 2 m-\mu$ and $\mu$ is the chemical potential. Simply factorising the averages in Eq.(2.23) and using the above equation reproduces the well known Drude result for the conductivity [12]. The quantum mechanical weak localization correction arises from correlations between retarded and advanced functions. The reason that there exist a large contribution from such disorder-correlation is of course the possible coherence between a trajectory and its time-reversed counterpart. Diagrammatically this is the famous Cooperon or weak localization (WL) correction to the conductivity shown in Fig.2.1. In $d \leq 2$ this diagram gives the leading correction to the classical Drude result. It is evaluated using the standard Feynman rules which yield

$$
\begin{gathered}
\bar{\sigma}_{W L}(\omega)=\frac{e^{2}}{2 \omega d m^{2} \mathcal{V}} \sum_{\mathbf{k}, \mathbf{q}} \mathbf{k} \cdot \mathbf{q} \int_{-\infty}^{\infty} \frac{d \varepsilon}{2 \pi}[h(\omega+\varepsilon)-h(\varepsilon)]{\overline{\mathcal{G}^{0}}}_{\mathbf{k}}^{R}(\omega+\varepsilon){\overline{\mathcal{G}^{0}}}_{\mathbf{q}}^{R}(\omega+\varepsilon) \times \\
{\overline{\mathcal{G}^{0}}}_{\mathbf{k}}^{A}(\varepsilon) \overline{\mathcal{G}}_{\mathbf{q}}^{A}(\varepsilon) \mathrm{C}_{n i}(\mathbf{k}+\mathbf{q} ; \omega) .
\end{gathered}
$$

Since in disordered metals the electron motion is diffusive, the dominant contribution to the conductivity comes from the range with momenta $|\mathbf{k}| \ll 1 / l$, where $l$ is the elastic mean free path, and frequencies $|\omega| \ll 1 / \tau_{e l}$. In this limit the ladder diagrams defining the non-interacting Cooperon in Fig.2.1(b) can be evaluated, resulting in 13

$$
\mathrm{C}_{n i}(\mathbf{k} ; \omega)=\frac{\Delta}{2 \pi \tau_{e l}^{2}} \frac{1}{D \mathbf{k}^{2}-i \omega} .
$$

Substituting Eq.(2.26) into Eq.(2.25) the $\mathbf{k}+\mathbf{q}=\mathbf{0}$ mode obviously gives a divergent WL-correction in the limit $\omega \rightarrow 0$. To cure this singularity for free particles, nonperturbative methods have to be employed. Performing a supersymmetric $\sigma$-model 
a)

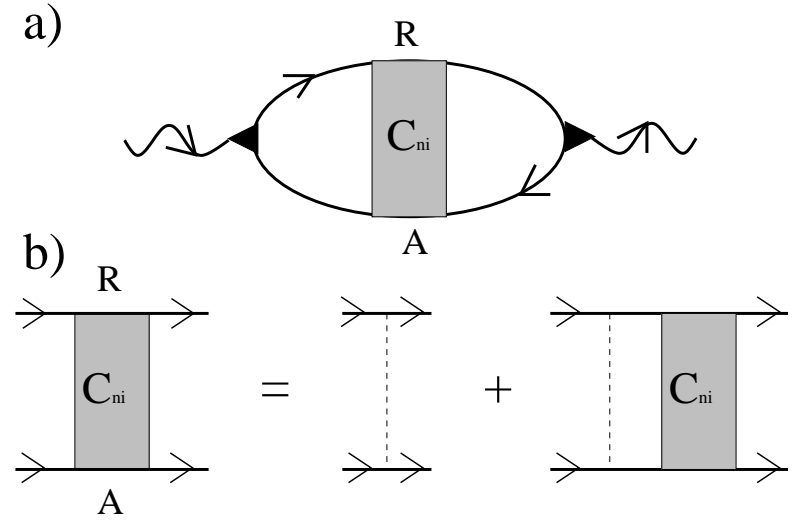

Figure 2.1: Diagrammatic representation of the weak localization correction to the conductivity. a: Conductivity bubble with a single Cooperon (shaded box). Directed lines symbolize averaged Green's functions as defined in Eq.(2.24) and the wiggled lines ending in a filled triangle represent current-vertices. $b$ : Bethe-Salpeter equation for the non-interacting Cooperon $\mathrm{C}_{n i}$. The thin dashed lines denote disorder scattering.

calculation Efetov has shown [14], that a cutoff $\Delta / \pi$ has to be introduced in the denominator of the Cooperon. Since the average level spacing $\Delta$ scales to zero for $\mathcal{V} \rightarrow \infty$ this is a finite size effect. In the following we will thus use the expression

$$
\mathrm{C}_{n i}(\mathbf{k} ; \omega)=\frac{\Delta}{2 \pi \tau_{e l}^{2}} \frac{1}{D \mathbf{k}^{2}-i \omega+\frac{\Delta}{\pi}}
$$

instead of Eq.(2.26) for the non-interacting Cooperon. The Cooperon gives a dominant contribution for small momenta $|\mathbf{k}+\mathbf{q}|<1 / l$ and frequencies $|\omega| \ll 1 / \tau_{e l}$. Since the averaged Green's functions are dominated by momenta of the order $k_{F} \gg 1 / l$, one can approximate $\mathbf{q} \simeq-\mathbf{k}$ in their arguments and in the scalar product in Eq.(2.25). For frequencies $|\omega| \ll 1 / \tau_{e l}$ the frequency dependence of the Green's functions can also be neglected compared to the cutoff $1 / \tau_{e l}$. This yields

$$
\bar{\sigma}_{W L}(\omega)=-\frac{e^{2}}{2 \omega d m^{2} \mathcal{V}} \sum_{\mathbf{q}}|\mathbf{q}|^{2} \int_{-\infty}^{\infty} \frac{d \varepsilon}{2 \pi}[h(\omega+\varepsilon)-h(\varepsilon)]\left|\overline{\mathcal{G}}_{\mathbf{q}}^{R}(0)\right|^{4} \sum_{\mathbf{k}} \mathrm{C}_{n i}(\mathbf{k} ; \omega) .
$$

Now since $\overline{\mathcal{G}}_{\mathbf{q}}(0)$ is dominated by momenta $|\mathbf{q}| \simeq k_{F}$ the approximation $|\mathbf{q}|^{2} \simeq k_{F}^{2}$ is made. The $\mathbf{q}$ sum is evaluated assuming a constant, disorder independent density of states $\nu_{d}(\varepsilon)$ in the vicinity of the Fermi energy,

$$
\overline{\nu_{d}(\varepsilon)}=\frac{1}{L^{d}} \sum_{\alpha} \overline{\delta\left(\varepsilon-\varepsilon_{\alpha}+\mu\right)} \approx \overline{\nu_{d}(0)} \approx \frac{1}{L^{d}} \sum_{\mathbf{q}} \delta\left(\xi_{\mathbf{q}}\right) .
$$


$\varepsilon_{\alpha}$ are the exact eigenvalues for a given realization of disorder. In the last approximation $\overline{\nu_{d}(0)}=: \nu_{d}$ is replaced by its value in the absence of disorder. $\nu_{d}=1 /\left(L^{d} \Delta\right)$ is the average density of states at the Fermi energy. 1 One can then rewrite the q-sum Eq.(2.28) as

$$
\sum_{\mathbf{q}}\left|\overline{\mathcal{G}}_{\mathbf{q}}^{R}(0)\right|^{4}=\int_{-\infty}^{\infty} d \xi \sum_{\mathbf{q}} \delta\left(\xi_{\mathbf{q}}-\xi\right) \frac{1}{\left(\xi^{2}+\frac{1}{\left(2 \tau_{e l}\right)^{2}}\right)^{2}} \approx \frac{1}{\Delta} \int_{-\infty}^{\infty} d \xi \frac{1}{\left(\xi^{2}+\frac{1}{\left(2 \tau_{e l}\right)^{2}}\right)^{2}}
$$

Solving the $\varepsilon$ and $\xi$ integral in Eq.(2.28) and (2.30) respectively finally yields

$$
\bar{\sigma}_{W L}(\omega)=-\frac{\sigma_{d}}{\pi \nu_{d}} \frac{1}{L^{d}} \sum_{|\mathbf{k}|<1 / l} \frac{2 \pi \tau_{e l}^{2}}{\Delta} \mathrm{C}_{n i}(\mathbf{k} ; \omega) .
$$

$\sigma_{d}$ is the classical Drude conductivity in $d$ dimensions, which is related to the density of states via the Einstein relation $\sigma_{d}=e^{2} \nu_{d} D$. In Eq.(2.31) the factor $1 / \mathcal{V}$ is replaced by $1 / L^{d}$, since in quasi $d$-dimensional samples with $\mathcal{V}=a^{3-a} L^{d}$ the ballistic transverse modes with $|\mathbf{k}|>1 / l$ have to be integrated out, yielding a factor $a^{3-d}$. The Cooperon is sometimes defined without the prefactor $\Delta /\left(2 \pi \tau_{e l}^{2}\right)$ since it cancels in the expression for $\bar{\sigma}_{W L}$. However, evaluating the ladder diagrams of Fig.2.1(b) leads to this factor and for diagrammatic calculations it is therefore easier to use the Cooperon as defined in Eq.(2.27). This form of the Cooperon is commonly used in mesoscopic physics. In the limit $\mathcal{V} \rightarrow 0$ the average level spacing vanishes, so that for macroscopic systems the definition (2.26) is usually multiplied by a factor $\mathcal{V}$, replacing $\Delta$ by $1 / \nu_{d}$. Which definition to use is a matter of convenience and the rescaled form will also be introduced later on. In the limit $\mathcal{V} \rightarrow \infty$ (i.e. $\Delta \rightarrow 0$ ) where the $\mathbf{k}$-sum is replaced by an integral according to $1 / L^{d} \sum_{\mathbf{k}} \rightarrow \int d \mathbf{k} /(2 \pi)^{d}$, the WL-correction is still divergent in $d \leq 2$. Physical arguments are used to handle this problem. In realistic systems the electrons are not completely free particles. There will always be interactions which lead to dephasing. All processes that break the time-reversal invariance suppress coherent backscattering and thus WL. On a phenomenological level this can be modeled by simply introducing an additional dephasing cutoff $1 / \tau_{\varphi}$ into the Cooperon propagator. This is just the inverse dephasing time that was already introduced in the semiclassical path-integral description. In other words, one assumes, that in an interacting system the Cooperon has the form

$$
\mathrm{C}(\mathbf{k} ; \omega)=\frac{\Delta}{2 \pi \tau_{e l}^{2}} \frac{1}{D \mathbf{k}^{2}-i \omega+\frac{1}{\tau_{\varphi}}} .
$$

\footnotetext{
${ }^{1}$ According to the Fermi liquid theory only quasiparticles with energy close to the Fermi energy contribute to the electronic transport and thus only a small part of the spectrum where $\nu_{d}(\varepsilon)$ is approximately constant should contribute to $\bar{\sigma}_{W L}(\omega)$. However, in a free electron gas the levels are strongly correlated over the whole spectrum [16] and the truncation can only be justified by physical arguments.
} 
The WL-correction for such systems is obtained by simply replacing $\mathrm{C}_{n i}$ in Eq.(2.31) by C. Transforming back to the time-variable $t$,

$$
\bar{\sigma}_{W L}(t)=-\Theta(t) \frac{\sigma_{d}}{\pi \nu_{d}} \frac{1}{L^{d}} \sum_{|\mathbf{k}|<1 / l} e^{-\left(D \mathbf{k}^{2}+\frac{1}{\tau_{\varphi}}\right) t},
$$

one sees, that $\tau_{\varphi}$ is the time-scale that cuts off the time dependence of the WLcorrection. This is equivalent to the semiclassical argument, that diffusive paths which are longer than $L_{\varphi}=\sqrt{D \tau_{\varphi}}$ do not contribute to coherent backscattering. Since an external magnetic field $\mathbf{B}$ directly affects the electrons phase, the WLcorrection is also very sensitive to such fields. Detailed derivations of $\bar{\sigma}_{W L}(\mathbf{B})$ can be found in the literature [17, 5, 4]. Experimentally the DC $(\omega=0)$ conductivity is measured as a function of $\mathbf{B}$ and fit to the theoretical curve for $\bar{\sigma}_{W L}(\mathbf{B})$ by adjusting the value of $\tau_{\varphi}[18,19,45]$. The obtained agreement between experiment and theory is striking and the phase coherence time can be obtained with a high accuracy. From such a measurement it is however a priory not clear, which effects led to the observed dephasing. Under realistic conditions there are various phenomena that contribute to $\tau_{\varphi}$. The most important ones are electron-electron and electron-phonon interactions, magnetic impurities (i.e. isolated ions with permanent magnetic moment), and external electromagnetic radiation. To calculate the dephasing time one has to include dephasing effects on a microscopic level, e.g. add an external fluctuating field to the Hamiltonian (2.5). One then calculates the weak localization correction and extracts $\tau_{\varphi}$ from comparison with Eq.(2.33). In the following dephasing due to electron-electron interactions with small energy transfer and due to external time dependent electric fields will be investigated. Making use of the fluctuationdissipation theorem, both effects can be treated on equal footing [20]. To proceed further one thus needs an equation for the WL-correction in the presence of external dephasing fields. A detailed derivation is presented in the next section. 


\subsection{Weak localization in the presence of external dephasing fields}

In this section a well known equation first obtained by Altshuler et al. $[8]$ for the weak localization correction in the presence of dephasing fields will be rederived. Since two versions of this equation, which differ by a factor of two, appear in the literature [15] a detailed derivation is presented heret. The physical system considered here is a disordered metallic conductor in the presence of an external driving field $\mathbf{E}_{d r i}(\mathbf{r}, t)=$ $-(1 / c) \partial_{t} \mathbf{A}_{d r i}(\mathbf{r}, t)$ and an additional fluctuating longitudinal electric field $\mathbf{E}$ which is assumed to be so weak, that it leaves the electrons trajectories unchanged but only affects their phases. Introducing a scalar potential $V(\mathbf{r}, t):=(-e) \phi(\mathbf{r}, t)$ which is related to $\mathbf{E}$ via $\mathbf{E}(\mathbf{r}, t)=-\nabla_{\mathbf{r}} \phi(\mathbf{r}, t)$, the Hamiltonian of the system takes the form

$$
\hat{H}(t)=\sum_{i}\left\{\frac{1}{2 m}\left[\hat{\mathbf{P}}_{i}+\frac{e}{c} \mathbf{A}_{d r i}\left(\hat{\mathbf{R}}_{i}, t\right)\right]^{2}+U\left(\hat{\mathbf{R}}_{i}\right)+V\left(\hat{\mathbf{R}}_{i}, t\right)\right\} .
$$

In order to calculate the response to the driving field, the part containing $\mathbf{A}_{d r i}$ is separated off. This is just the term $\hat{H}_{1}(t)$ given in Eq.(2.11). The remaining part is then the sum of the time-independent part $\hat{H}_{0}$ (see Eq.(2.10) $)$ and the timedependent field $\hat{V}(t)$,

$$
\begin{aligned}
& \hat{H}_{0, V}(t)=\hat{H}_{0}+\hat{V}(t) \\
&=\sum_{\mathbf{q}} \frac{\mathbf{q}^{2}}{2 m} \hat{\Psi}_{\mathbf{q}}^{\dagger} \hat{\Psi}_{\mathbf{q}}+\int d \mathbf{r}[U(\mathbf{r})+V(\mathbf{r}, t)] \hat{\Psi}^{\dagger}(\mathbf{r}) \hat{\Psi}(\mathbf{r}), \\
& \hat{H}_{1}(t)=\frac{e}{2 m c \mathcal{V}} \sum_{\mathbf{q}, \mathbf{q}^{\prime}} \mathbf{A}_{d r i}\left(\mathbf{q}-\mathbf{q}^{\prime}, \omega\right) \cdot\left[\mathbf{q}+\mathbf{q}^{\prime}\right] \hat{\Psi}_{\mathbf{q}}^{\dagger} \hat{\Psi}_{\mathbf{q}^{\prime}}+\mathcal{O}\left(A_{d r i}^{2}\right),
\end{aligned}
$$

and the total Hamiltonian is

$$
\hat{H}(t)=\hat{H}_{0, V}(t)+\hat{H}_{1}(t) .
$$

Note that now $H_{1}$ and $H_{0, V}$ are time-dependent. To derive an expression for the current in the presence of fluctuating external fields it is convenient to use the Keldysh technique. Using Eq.(A.3) for the paramagnetic current density and Eq.(2.14) for the time-dependent perturbation $\hat{H}_{1}(t)$ it is straightforward to obtain the current to linear order in $\mathbf{A}_{d r i}$ from the general expression Eq.(A.15). One gets

$$
\begin{gathered}
\delta \mathbf{j}_{\text {para }}(t)=\frac{i}{c \mathcal{V}}\left(\frac{e}{2 m}\right)^{2} \sum_{\mathbf{q}, \mathbf{p}, \mathbf{p}^{\prime}} \int_{-\infty}^{\infty} d t_{1} \mathbf{q}\left[\mathbf{A}_{d r i}\left(\mathbf{p}-\mathbf{p}^{\prime}, t_{1}\right) \cdot\left(\mathbf{p}+\mathbf{p}^{\prime}\right)\right]\left[\mathcal{G}_{\mathbf{q}, \mathbf{p}}^{R}\left(t, t_{1}\right) \mathcal{G}_{\mathbf{p}^{\prime}, \mathbf{q}}^{K}\left(t_{1}, t\right)\right. \\
\left.+\mathcal{G}_{\mathbf{q}, \mathbf{p}}^{K}\left(t, t_{1}\right) \mathcal{G}_{\mathbf{p}^{\prime}, \mathbf{q}}^{A}\left(t_{1}, t\right)\right] .
\end{gathered}
$$

\footnotetext{
${ }^{2}$ In a footnote Eiler [15] pointed out that there is a factor of two missing in the equation for the Cooperon in an external field derived by Altshuler, Aronov and Khmelnitskii [8]. As will be shown Eiler's criticism is indeed correct. Unfortunately, Eiler's paper has not received much attention.
} 


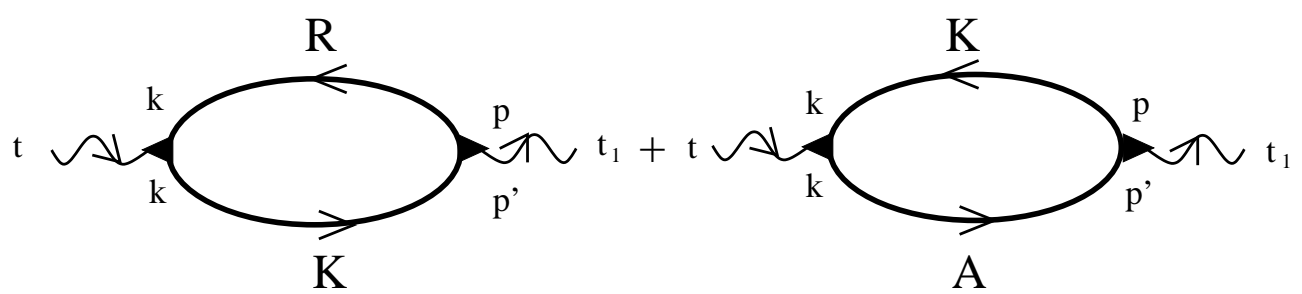

Figure 2.2: Diagrammatic representation of Eq.(2.38). The thick lines are the full Green's functions for the Hamiltonian $H_{0, V}(t)$. They include disorder and the timedependent field $V(\mathbf{r}, t)$. The wiggled lines with filled triangles are current-vertices.

Now $\mathcal{G}^{R / A}$ are the full Green's functions according to the time-dependent Hamiltonian $\hat{H}_{0, V}(t)$. A diagrammatic representation of this equation is shown in Fig.2.2. From the Dyson equation (A.9) for the Green's function in matrix form one obtains the corresponding equations for the components:

$$
\mathcal{G}_{\mathbf{k}, \mathbf{k}^{\prime}}^{R / A}\left(t, t^{\prime}\right)=\mathcal{G}_{\mathbf{k}, \mathbf{k}^{\prime}}^{0, R / A}\left(t, t^{\prime}\right)+\sum_{\mathbf{q}, \mathbf{q}^{\prime}} \int_{-\infty}^{\infty} d t_{1} \mathcal{G}_{\mathbf{k}, \mathbf{q}}^{0, R / A}\left(t, t_{1}\right) V_{\mathbf{q}, \mathbf{q}^{\prime}}\left(t_{1}\right) \mathcal{G}_{\mathbf{q}^{\prime}, \mathbf{k}^{\prime}}^{R / A}\left(t_{1}, t^{\prime}\right)
$$

and

$$
\begin{aligned}
\mathcal{G}_{\mathbf{k}, \mathbf{k}^{\prime}}^{K}\left(t, t^{\prime}\right)=\mathcal{G}_{\mathbf{k}, \mathbf{k}^{\prime}}^{0, K}\left(t, t^{\prime}\right)+\sum_{\mathbf{q}, \mathbf{q}^{\prime}} \int_{-\infty}^{\infty} & d t_{1}\left[\mathcal{G}_{\mathbf{k}, \mathbf{q}}^{0, R}\left(t, t_{1}\right) V_{\mathbf{q}, \mathbf{q}^{\prime}}\left(t_{1}\right) \mathcal{G}_{\mathbf{q}^{\prime}, \mathbf{k}^{\prime}}^{K}\left(t_{1}, t^{\prime}\right)\right. \\
& \left.+\mathcal{G}_{\mathbf{k}, \mathbf{q}}^{0, K}\left(t, t_{1}\right) V_{\mathbf{q}, \mathbf{q}^{\prime}}\left(t_{1}\right) \mathcal{G}_{\mathbf{q}^{\prime}, \mathbf{k}^{\prime}}^{A}\left(t_{1}, t^{\prime}\right)\right]
\end{aligned}
$$

The $\mathcal{G}^{0}$ are the Green's functions for a system described by the time-independent Hamiltonian $\hat{H}_{0}$. They do not contain the fluctuating external field $V(\mathbf{r}, t)$ but still the static disorder potential $U(\mathbf{r})$. These equations can now be used to calculate the current in the presence of an external field. As an example Fig.2.3 shows all diagrams that are obtained from the first diagram in Fig.2.2 if $\mathcal{G}^{R}$ is expanded to first, and $\mathcal{G}^{K}$ to second order in the field. The equilibrium function $\mathcal{G}^{0, K}$ is related to the corresponding retarded and advanced function via Eq.(A.10). Translating the diagrams into frequency-space is easily done, using the standard Feynman rules. If the external field $V$ is time-independent, frequency is conserved at each fieldvertex. As a result, in this case all electron-lines on one side of the conductivity bubble (where the upper side belongs to $\mathcal{G}^{R}$ and the lower one to $G^{K}$ ) have the same frequency. Using Eq. (A.10) one sees, that now only the terms $\mathcal{G}^{0, R} \mathcal{G}^{0, R} \mathcal{G}^{0, R}$ and $\mathcal{G}^{0, A} \mathcal{G}^{0, A} \mathcal{G}^{0, A}$ contribute to the expansion of $\mathcal{G}^{K}$. All other terms cancel. This must of course be the case, since in equilibrium Eq. A.10) can directly be applied to $\mathcal{G}^{K}$. The situation under investigation is a time-dependent field, which affects the electrons phase, but is too weak to drive them out of equilibrium, i.e. it leaves the distribution function unchanged. In this case it is justified to retain the analytic equilibrium 

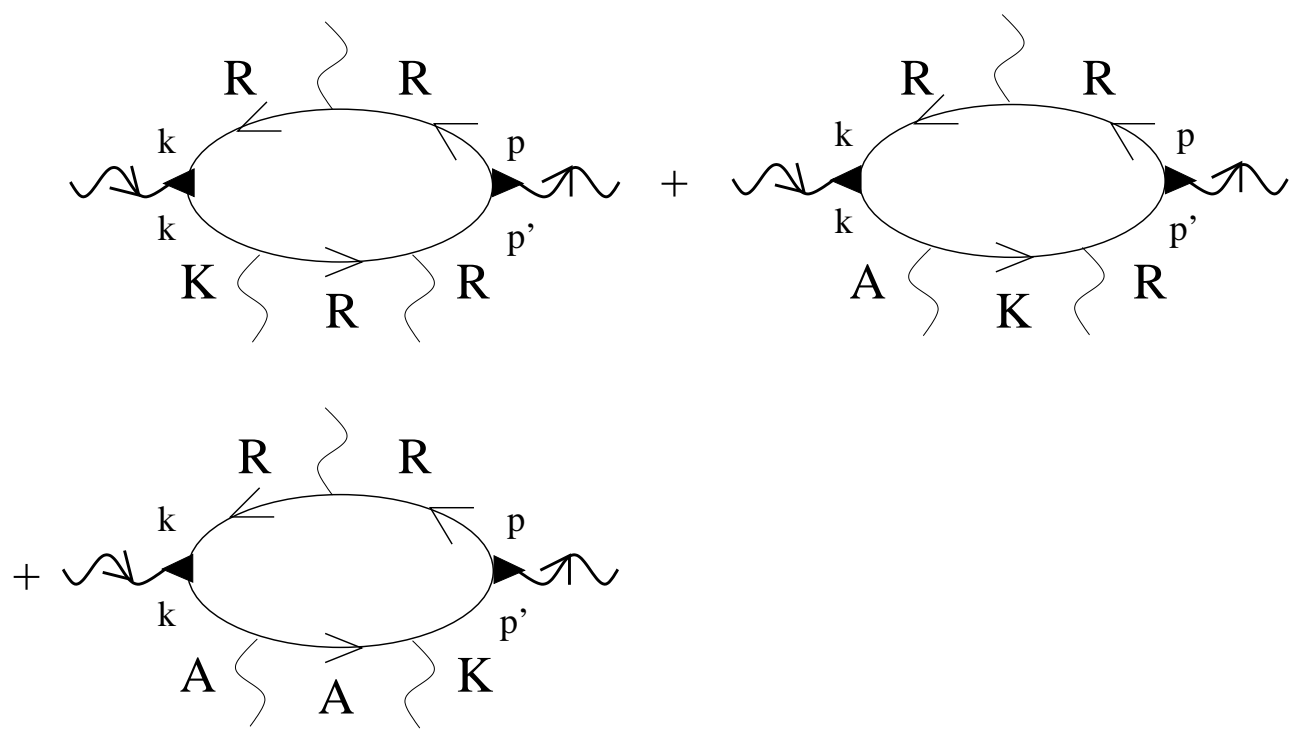

Figure 2.3: Diagrams obtained by expanding $\mathcal{G}^{R}$ to first, and $G^{K}$ to second order in the potential $V$. The thin lines are the equilibrium Green's functions $\mathcal{G}^{0}$ for the Hamiltonian $H_{0}$. Thin wiggled lines represent the field $V(t)$.

structure consisting of only retarded or only advanced functions on each side of the bubble. Without the external field $V(t)$ the weak localization correction is given by the single Cooperon diagram shown in Fig.2.1. To take dephasing effects that are not caused by $V(t)$ into account, e.g. electron-phonon interactions or magnetic impurities, a cutoff $\Gamma_{0}$ is introduced on a phenomenological level and $\mathrm{C}_{n i}$ is replaced by $\mathrm{C}_{0}$, with

$$
\mathrm{C}_{0}(\mathbf{Q} ; \varepsilon)=\frac{\Delta}{2 \pi \tau_{e l}^{2}} \frac{1}{D \mathbf{Q}^{2}-i \varepsilon+\Gamma_{0}}
$$

Since the disorder is static, the Cooperon $\mathrm{C}_{0}$ conserves the frequency on each electron line. In the presence of a fluctuating field, this will no longer be the case. The Bethe-Salpeter equation for the Cooperon has to be generalized, to include interactions with $V(t)$. Diagrammatically this is done by attaching interaction lines to the electron propagators in Fig.2.1(b). This leads to the generalized Bethe-Salpeter equation for the Cooperon which is shown in Fig.2.4. Here the interaction is only included to first order in each electron propagator. Still infinite orders in the interaction are summed up due to the self consistency contained in the diagrammatic equation 2.4. The corresponding integral-equation is 


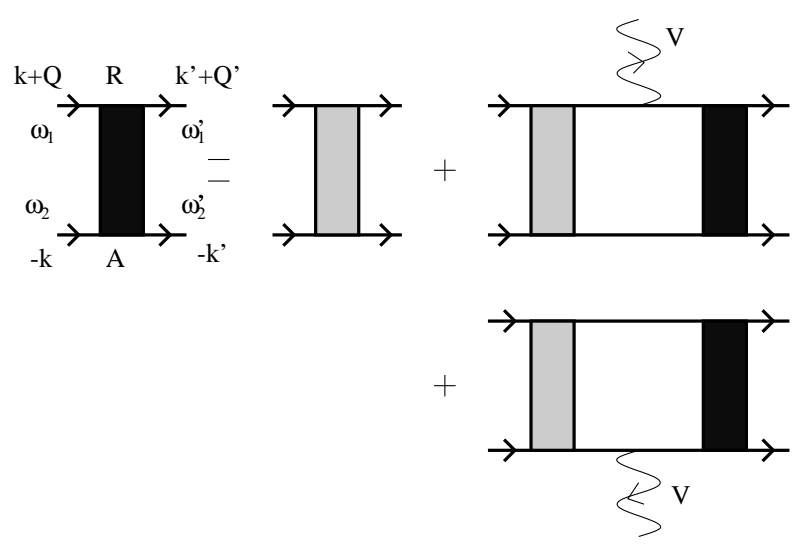

Figure 2.4: Diagrammatic representation of the Cooperon in an external field. The dark boxes represent the full Cooperon $\tilde{\mathrm{C}}$ and the shaded boxes are the Cooperon without interactions, $\mathrm{C}_{0}$. Wiggled lines indicate the external potential $V_{p}(\tilde{\omega})$. The upper electron-line is retarded, and the lower advanced.

$$
\begin{aligned}
& \tilde{\mathrm{C}}\left(\mathbf{Q}, \mathbf{Q}^{\prime} ; \omega_{1}, \omega_{1}^{\prime}, \omega_{2}, \omega_{2}^{\prime}\right)=\mathcal{V}(2 \pi)^{2} \delta_{\mathbf{Q}, \mathbf{Q}^{\prime}} \delta\left(\omega_{1}-\omega_{1}^{\prime}\right) \delta\left(\omega_{2}-\omega_{2}^{\prime}\right) \mathbf{C}_{0}\left(\mathbf{Q} ; \omega_{1}-\omega_{2}\right) \\
& +\frac{1}{\mathcal{V}} \sum_{\mathbf{q}, \mathbf{p}} \int_{-\infty}^{\infty} \frac{d \tilde{\omega}}{2 \pi}\left[\mathrm{C}_{0}\left(\mathbf{Q} ; \omega_{1}-\omega_{2}\right){\overline{\mathcal{G}^{0}}}_{\mathbf{q}+\mathbf{Q}}^{R}\left(\omega_{1}\right) V_{\mathbf{p}}(\tilde{\omega}) \overline{\mathcal{G}}_{\mathbf{q}+\mathbf{Q}-\mathbf{p}}^{R}\left(\omega_{1}-\tilde{\omega}\right) \overline{\mathcal{G}}_{\mathbf{q}}^{A}\left(\omega_{2}\right) \times\right. \\
& \tilde{\mathbf{C}}\left(\mathbf{Q}-\mathbf{p}, \mathbf{Q}^{\prime} ; \omega_{1}-\tilde{\omega}, \omega_{1}^{\prime}, \omega_{2}, \omega_{2}^{\prime}\right)+\mathrm{C}_{0}\left(\mathbf{Q} ; \omega_{1}-\omega_{2}\right) \overline{\mathcal{G}}_{\mathbf{q}+\mathbf{Q}}^{R}\left(\omega_{1}\right) V_{\mathbf{p}}(\tilde{\omega}) \times \\
& \left.{\overline{\mathcal{G}^{0}}}_{-\mathbf{q}}^{A}\left(\omega_{2}\right) \overline{\mathcal{G}}_{-\mathbf{q}-\mathbf{p}}^{A}\left(\omega_{2}-\tilde{\omega}\right) \tilde{\mathrm{C}}\left(\mathbf{Q}-\mathbf{p}, \mathbf{Q}^{\prime} ; \omega_{1}, \omega_{1}^{\prime}, \omega_{2}-\tilde{\omega}, \omega_{2}^{\prime}\right)\right] \text {. }
\end{aligned}
$$

Here $\mathcal{V}$ is the volume which for quasi $d$-dimensional systems is assumed to be of the form $\mathcal{V}=L^{d} a^{3-d}$ and in true $d$-dimensions reduces to $\mathcal{V}=L^{d}$. To treat both situations on equal footing, a factor $a^{2} \delta_{k_{2}, 0} \delta_{k_{3}, 0}$ for the quasi one-dimensional case and $a \delta_{k_{3}, 0}$ for quasi two dimensions is implicitly assumed with each momentum $\mathbf{k}$ appearing in the argument of a Cooperon $\left(k_{i}\right.$ are the Cartesian components of $\mathbf{k}$ which represents either $\mathbf{Q}, \mathbf{Q}^{\prime}$ or $\mathbf{Q}-\mathbf{p}$ ). This just states that there is no diffusion in the transverse channels and $d$ is the effective dimension regarding diffusion. The impurity-averaged Green's functions are given by

$$
{\overline{\mathcal{G}^{0}}}_{\mathbf{k}}^{R / A}(\omega)=\frac{1}{\omega-\left(\frac{\mathbf{k}^{2}}{2 m}-\mu\right) \pm \frac{i}{2 \tau_{e l}}}
$$

The Cooperon is dominated by frequencies much smaller than $1 / \tau_{e l}$ and momenta smaller than $1 / l$. Therefore the frequency-dependence of the Green's functions can be neglected and one can set $\mathbf{Q} \approx 0, \mathbf{Q}-\mathbf{p} \approx 0$ in the argument of Green's functions. Within these approximations the sum over momenta $\mathbf{q}$ can be evaluated. Again the 
approximations in Eq.(2.29) and (2.30) are used:

$$
\sum_{\mathbf{q}}\left[{\overline{\mathcal{G}^{0}}}_{\mathbf{q}}^{R}(0)\right]^{2}{\overline{\mathcal{G}^{0}}}_{\mathbf{q}}^{A}(0) \approx \frac{1}{\Delta} \int_{-\infty}^{\infty} d \xi \frac{1}{\left(-\xi+\frac{i}{2 \tau_{e l}}\right)^{2}\left(-\xi-\frac{i}{2 \tau_{e l}}\right)}=-\frac{2 \pi i \tau_{e l}^{2}}{\Delta}
$$

The q-sum in the second term in braces in Eq.(2.42) is just the complex conjugate of Eq.(2.44). Keeping in mind, that the diffusive approximation is only valid on distances much lager than $l$, the ballistic modes of the $\mathbf{p}$-sum appearing in the quasi $d$-dimensional case can also be integrated out. This merely replaces the three dimensional potential $V_{\mathbf{p}}$ by an effective $d$-dimensional one and the factor $1 / \mathcal{V}$ by $1 / L^{d}$. Since there is now no explicit dependence on transverse modes anymore, the implicit dependence due to the factors $a^{3-d} \delta_{k_{i}, 0}$ can also be dropped and all momenta are treated as truely $d$-dimensional in the following. From Eq.(2.42) one obtains

$$
\begin{aligned}
& \tilde{\mathrm{C}}\left(\mathbf{Q}, \mathbf{Q}^{\prime} ; \omega_{1}, \omega_{1}^{\prime}, \omega_{2}, \omega_{2}^{\prime}\right)=L^{d}(2 \pi)^{2} \delta_{\mathbf{Q}, \mathbf{Q}^{\prime}} \delta\left(\omega_{1}-\omega_{1}^{\prime}\right) \delta\left(\omega_{2}-\omega_{2}^{\prime}\right) \mathbf{C}_{0}\left(\mathbf{Q} ; \omega_{1}-\omega_{2}\right) \\
& -\frac{1}{L^{d}} \frac{2 \pi i \tau_{e l}^{2}}{\Delta} \sum_{\mathbf{p}}^{\prime} \int_{-\infty}^{\infty} \frac{d \tilde{\omega}}{2 \pi}\left[\mathbf{C}_{0}\left(\mathbf{Q} ; \omega_{1}-\omega_{2}\right) V_{\mathbf{p}}(\tilde{\omega}) \tilde{\mathbf{C}}\left(\mathbf{Q}-\mathbf{p}, \mathbf{Q}^{\prime} ; \omega_{1}-\tilde{\omega}, \omega_{1}^{\prime}, \omega_{2}, \omega_{2}^{\prime}\right)\right. \\
& \left.\quad-\mathrm{C}_{0}\left(\mathbf{Q} ; \omega_{1}-\omega_{2}\right) V_{\mathbf{p}}(\tilde{\omega}) \tilde{\mathbf{C}}\left(\mathbf{Q}-\mathbf{p}, \mathbf{Q}^{\prime} ; \omega_{1}, \omega_{1}^{\prime}, \omega_{2}-\tilde{\omega}, \omega_{2}^{\prime}\right)\right]
\end{aligned}
$$

$\mathbf{Q}, \mathbf{Q}^{\prime}$ and $\mathbf{p}$ are now $d$-dimensional vectors. The prime on the $\mathbf{p}$-sum indicates, that all transverse modes are integrated out and the summation is restricted to diffusive modes. $\Delta$ is the average level-spacing at the Fermi energy, connected to the $d$-dimensional density of states $\nu_{d}$ by $\Delta=1 /\left(\nu_{d} L^{d}\right)$. In the quasi $d$-dimensional case $\nu_{d}$ is related to the three dimensional density of states via $\nu_{d}=\nu_{3} a^{3-d}$. Since $\mathrm{C}_{0}$ depends only on a single frequency, the full Cooperon $\tilde{\mathrm{C}}$ can depend only on the difference between the incoming frequencies (see Fig.2.4),

$$
\varepsilon:=\omega_{1}-\omega_{2},
$$

the difference between the outgoing frequencies,

$$
\varepsilon^{\prime}:=\omega_{1}^{\prime}-\omega_{2}^{\prime},
$$

and the total frequency transfered to the field,

$$
\omega:=\omega_{1}+\omega_{2}-\left(\omega_{1}^{\prime}+\omega_{2}^{\prime}\right)
$$

Physically this just states that the Cooperon is unaffected if all frequencies are shifted by the same amount. Using this fact and defining

$$
\tilde{\mathrm{C}}\left(\mathbf{Q}, \mathbf{Q}^{\prime} ; \omega_{1}, \omega_{1}^{\prime}, \omega_{2}, \omega_{2}^{\prime}\right)=: \mathbf{C}\left(\mathbf{Q}, \mathbf{Q}^{\prime} ; \omega_{1}-\omega_{2}, \omega_{1}^{\prime}-\omega_{2}^{\prime}, \omega_{1}+\omega_{2}-\left(\omega_{1}^{\prime}+\omega_{2}^{\prime}\right)\right)
$$


Eq.(2.45) becomes

$$
\begin{array}{r}
\mathrm{C}\left(\mathbf{Q}, \mathbf{Q}^{\prime} ; \varepsilon, \varepsilon^{\prime}, \omega\right)= \\
-\frac{1}{L^{d}} \frac{2 \pi i \tau_{e l}^{2}}{\Delta} \sum_{\mathbf{p}}^{\prime} \int_{-\infty}^{\infty} \frac{d \tilde{\omega}}{2 \pi}\left[\mathrm{C}_{0}(\mathbf{Q} ; \varepsilon) V_{\mathbf{p}}(\tilde{\omega}) \mathrm{C}\left(\mathbf{Q}-\mathbf{p}, \mathbf{Q}^{\prime} ; \varepsilon-\tilde{\mathbf{Q}, \mathbf{Q}^{\prime}}, \varepsilon^{\prime}, \omega-\tilde{\omega}\right)\right. \\
\left.-\mathrm{C}_{0}(\mathbf{Q} ; \varepsilon) V_{\mathbf{p}}(\tilde{\omega}) \mathrm{C}\left(\mathbf{Q}-\mathbf{p}, \mathbf{Q}^{\prime} ; \varepsilon+\tilde{\omega}, \varepsilon^{\prime}, \omega-\tilde{\omega}\right)\right] .
\end{array}
$$

Note that $\omega_{1}-\omega_{1}^{\prime}=(1 / 2)\left(\omega+\varepsilon-\varepsilon^{\prime}\right)$ and $\omega_{2}-\omega_{2}^{\prime}=(1 / 2)\left(\omega-\varepsilon+\varepsilon^{\prime}\right)$ which are just the arguments of the $\delta$-functions. Now the explicit form of $\mathrm{C}_{0}$ given in Eq.(2.41) is inserted and the whole Eq. (2.50) is multiplied by $D \mathrm{Q}^{2}-i \varepsilon+\Gamma_{0}$. Fourier transforming the resulting expression to space-time coordinates according to

$$
\mathrm{C}\left(\mathbf{r}, \mathbf{r}^{\prime} ; t, t^{\prime}, t_{0}\right)=\frac{1}{L^{2 d}} \sum_{\mathbf{Q}, \mathbf{Q}^{\prime}} \int_{-\infty}^{\infty} \frac{d \varepsilon d \varepsilon^{\prime} d \omega}{(2 \pi)^{3}} \mathrm{C}\left(\mathbf{Q}, \mathbf{Q}^{\prime} ; \varepsilon, \varepsilon^{\prime}, \omega\right) e^{i\left(\mathbf{Q} \cdot \mathbf{r}-\mathbf{Q}^{\prime} \cdot \mathbf{r}^{\prime}\right)} e^{-i\left(t \varepsilon-t^{\prime} \varepsilon^{\prime}+t_{0} \omega\right)},
$$

finally leads to the partial-differential equation for the Cooperon in an longitudinal external field:

$$
\begin{array}{r}
\left(D \hat{\mathbf{P}}_{\mathbf{r}}^{2}+\partial_{t}+\Gamma_{0}\right) \mathrm{C}\left(\mathbf{r}, \mathbf{r}^{\prime} ; t, t^{\prime}, t_{0}\right)=2 \frac{\Delta}{2 \pi \tau_{e l}^{2}} \delta\left(\mathbf{r}-\mathbf{r}^{\prime}\right) \delta\left(t-t^{\prime}\right) \\
-i\left[V\left(\mathbf{r}, t_{0}+t\right)-V\left(\mathbf{r}, t_{0}-t\right)\right] \mathrm{C}\left(\mathbf{r}, \mathbf{r}^{\prime} ; t, t^{\prime}, t_{0}\right),
\end{array}
$$

where $\hat{\mathbf{P}}_{\mathbf{r}}$ is the momentum operator. It is important to note the factor 2 from the transformation of the $\delta$-functions,

$$
\begin{aligned}
& \frac{1}{2 \pi} \int_{-\infty}^{\infty} d \varepsilon d \varepsilon^{\prime} d \omega \delta\left(\frac{\omega+\varepsilon-\varepsilon^{\prime}}{2}\right) \delta\left(\frac{\omega-\varepsilon+\varepsilon^{\prime}}{2}\right) e^{-i\left(t \varepsilon-t^{\prime} \varepsilon^{\prime}+t_{0} \omega\right)} \\
= & \frac{2}{2 \pi} \int_{-\infty}^{\infty} d \varepsilon d \varepsilon^{\prime} d \omega \delta\left(\frac{\omega+\varepsilon}{2}-\varepsilon^{\prime}\right) \delta\left(\frac{\omega-\varepsilon}{2}+\varepsilon^{\prime}\right) e^{-i\left(t \varepsilon-2 t^{\prime} \varepsilon^{\prime}+t_{0} \omega\right)} \\
= & \frac{2}{2 \pi} \int_{-\infty}^{\infty} d \varepsilon e^{-i\left(t-t^{\prime}\right) \varepsilon}=2 \delta\left(t-t^{\prime}\right) .
\end{aligned}
$$

Due to this factor the Cooperon without external field $V$ is connected to the Fouriertransformed of $\mathrm{C}_{0}(\mathbf{Q}, \varepsilon)$ by

$$
\mathrm{C}_{0}\left(\mathbf{r}, \mathbf{r}^{\prime} ; t, t^{\prime}, t_{0}\right)=2 \mathrm{C}_{0}\left(\mathbf{r}-\mathbf{r}^{\prime} ; t-t^{\prime}\right),
$$

where $\mathrm{C}_{0}\left(\mathbf{r}, \mathbf{r}^{\prime} ; t, t^{\prime}, t_{0}\right)$ is obtained from Eq.(2.52) by setting $V=0$ and

$$
\mathrm{C}_{0}(\mathbf{r} ; t)=\frac{1}{L} \sum_{\mathbf{Q}} \int_{-\infty}^{\infty} \frac{d \varepsilon}{2 \pi} \mathrm{C}_{0}(\mathbf{Q} ; \varepsilon) e^{i(\mathbf{Q} \cdot \mathbf{r}-t \varepsilon)}
$$


Note that the Cooperon with four time-variables,

$\tilde{\mathrm{C}}\left(\mathbf{r}, \mathbf{r}^{\prime} ; t_{1}, t_{1}^{\prime}, t_{2}, t_{2}^{\prime}\right)=\int_{-\infty}^{\infty} \frac{d \omega_{1} d \omega_{1}^{\prime} d \omega_{2} d \omega_{2}^{\prime}}{(2 \pi)^{4}} \tilde{\mathrm{C}}\left(\mathbf{r}, \mathbf{r}^{\prime} ; \omega_{1}, \omega_{1}^{\prime}, \omega_{2}, \omega_{2}^{\prime}\right) e^{-i\left(\omega_{1} t_{1}-\omega_{1}^{\prime} t_{1}^{\prime}+\omega_{2} t_{2}-\omega_{2}^{\prime} t_{2}^{\prime}\right)}$,

is now given by

$$
\tilde{\mathrm{C}}\left(\mathbf{r}, \mathbf{r}^{\prime} ; t_{1}, t_{1}^{\prime}, t_{2}, t_{2}^{\prime}\right)=\frac{1}{2} \delta\left(t_{1}+t_{2}-t_{1}^{\prime}-t_{2}^{\prime}\right) \mathrm{C}\left(\mathbf{r}, \mathbf{r}^{\prime} ; \frac{t_{1}-t_{2}}{2}, \frac{t_{1}^{\prime}-t_{2}^{\prime}}{2}, \frac{t_{1}+t_{2}}{2}\right) \text {. }
$$

It is convenient to introduce the rescaled Cooperon

$$
\mathcal{C}\left(\mathbf{r}, \mathbf{r}^{\prime} ; t, t^{\prime}, t_{0}\right):=\frac{2 \pi \tau_{e l}^{2}}{2 \Delta} \mathrm{C}\left(\mathbf{r}, \mathbf{r}^{\prime} ; t, t^{\prime}, t_{0}\right)
$$

Eq. (2.52) now reads

$$
\begin{gathered}
\left(D \hat{\mathbf{P}}_{\mathbf{r}}^{2}+\partial_{t}+\Gamma_{0}\right) \mathcal{C}\left(\mathbf{r}, \mathbf{r}^{\prime} ; t, t^{\prime}, t_{0}\right)=\delta\left(\mathbf{r}-\mathbf{r}^{\prime}\right) \delta\left(t-t^{\prime}\right) \\
-i\left[V\left(\mathbf{r}, t_{0}+t\right)-V\left(\mathbf{r}, t_{0}-t\right)\right] \mathcal{C}\left(\mathbf{r}, \mathbf{r}^{\prime} ; t, t^{\prime}, t_{0}\right)
\end{gathered}
$$

and the Cooperon without external field is normalized so that

$$
\mathcal{C}_{0}\left(\mathbf{r}, \mathbf{r}^{\prime} ; t, t^{\prime}, t_{0}\right)=\frac{1}{L^{d}} \sum_{\mathbf{Q}} \int_{-\infty}^{\infty} \frac{d \varepsilon}{2 \pi} \mathcal{C}_{0}(\mathbf{Q} ; \varepsilon) e^{i\left[\mathbf{Q}\left(\mathbf{r}-\mathbf{r}^{\prime}\right)-\varepsilon\left(t-t^{\prime}\right)\right]}=\mathcal{C}_{0}\left(\mathbf{r}-\mathbf{r}^{\prime} ; t-t^{\prime}\right),
$$

with

$$
\mathcal{C}_{0}(\mathbf{Q} ; \varepsilon)=\frac{1}{D \mathbf{Q}^{2}-i \varepsilon+\Gamma_{0}}
$$

In order to get rid of the factor 2 appearing in Eq.(2.54) the Cooperon without external field $\mathrm{C}_{0}$ had to be rescaled in a different way than $\mathrm{C}$ in Eq.(2.57), i.e. $\mathcal{C}_{0}=$ $\left(2 \pi \tau_{e l}^{2} / \Delta\right) \mathrm{C}_{0}$. It is emphasized that with the above normalization of the Cooperon (which seems to be quite natural) the time argument of the potentials in Eq.(2.58) is $t_{0} \pm t$. Note that in Ref. [8] the Cooperon has been normalized differently, and the times $t_{0} \pm t$ in the arguments of the potentials of Eq.(2.58) are replaced by $t_{0} \pm t / 2$. Although this apparent printing error in Ref. [8] has been pointed out by Eiler [15] many years ago, it continues to appear in the recent literature [23, 24, 25].

Eq.(2.58) for the Cooperon can now be used to calculate the weak localization correction to the conductivity, or the current respectively, in the presence of a fluctuating external field. The diagrammatic representation for the WL-correction to the current is shown in Fig.2.5. Translating these diagrams to an analyti- 


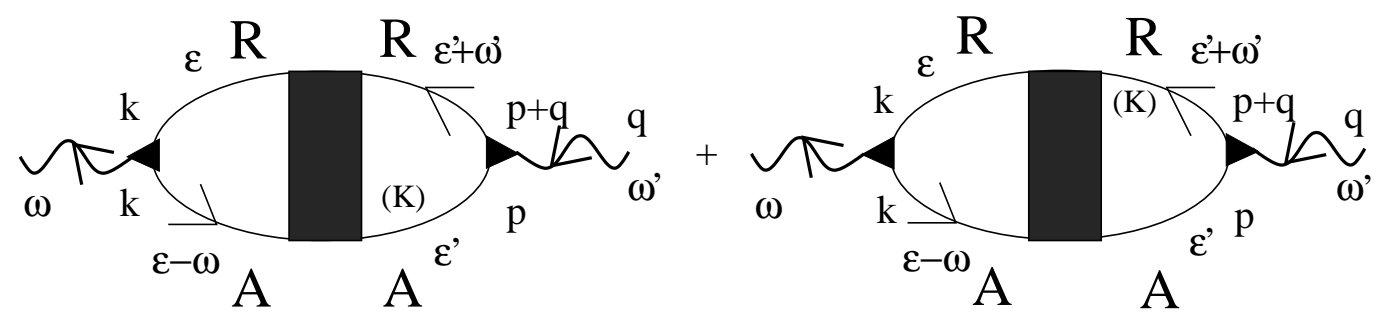

Figure 2.5: The weak localization correction to the current in the presence of a fluctuating external field $V(t)$. The dark box is the interacting Cooperon as diagrammatically defined in Fig.2.4. The Green's functions labeled by $(K)$ were obtained from the Keldysh component using Eq.(A.10).

cal expression yields for the spatially averaged (up to a factor $1 / \mathcal{V}$ ) component $\mathbf{j}_{W L}(\omega):=\mathbf{j}_{W L}(\mathbf{Q}=\mathbf{0}, \omega)$

$$
\begin{aligned}
\delta \mathbf{j}_{W L}(\omega)= & \frac{i}{c \mathcal{V}^{2}}\left(\frac{e}{2 m}\right)^{2} \sum_{\mathbf{k}, \mathbf{p}, \mathbf{q}} \int_{-\infty}^{\infty} \frac{d \varepsilon d \varepsilon^{\prime} d \omega^{\prime}}{(2 \pi)^{3}}\left[h\left(\varepsilon^{\prime}+\omega^{\prime}\right)-h\left(\varepsilon^{\prime}\right)\right]\left\{\mathbf{k}\left[2 \mathbf{A}_{d r i}\left(\mathbf{q}, \omega^{\prime}\right) \cdot \mathbf{p}\right] \times\right. \\
& \overline{\mathcal{G}}_{\mathbf{k}}^{R}(\varepsilon) \overline{\mathcal{G}}_{\mathbf{k}}^{A}(\varepsilon-\omega) \overline{\mathcal{G}}_{\mathbf{p}+\mathbf{q}}^{R}\left(\varepsilon^{\prime}+\omega^{\prime}\right) \overline{\mathcal{G}}^{A}{ }_{\mathbf{p}}^{A}\left(\varepsilon^{\prime}\right) \times \\
& \left.\mathrm{C}\left(\mathbf{k}+\mathbf{p}+\mathbf{q}, \mathbf{k}+\mathbf{p} ; \varepsilon^{\prime}+\omega^{\prime}-\varepsilon+\omega, \varepsilon-\varepsilon^{\prime}, \omega^{\prime}-\omega\right)\right\},
\end{aligned}
$$

with $h(\varepsilon):=\tanh (\beta \varepsilon / 2)$. The Cooperon is dominated by frequencies much smaller than the elastic cutoff $1 / \tau_{e l}$ in the Green's functions and the frequency dependence of the later can thus be neglected. Similarly the total momenta $\mathbf{k}+\mathbf{p}+\mathbf{q}$ and $\mathbf{k}+\mathbf{p}$ in Eq.(2.61) are restricted to values smaller than $1 / l$. Since the large contributions to the Green's functions come from momenta in the vicinity of the Fermi momentum one can set $\mathbf{k} \simeq-(\mathbf{p}+\mathbf{q}), \mathbf{k} \simeq-\mathbf{p}$ in their arguments. For an spatially homogeneous driving field $\mathbf{A}_{d r i}(\mathbf{Q}=0, \omega)=: \mathbf{A}_{d r i}(\omega)$ Eq.(2.61) now reduces to

$$
\begin{aligned}
\delta \mathbf{j}_{W L}(\omega)= & -\frac{2}{\mathcal{V}^{2}}\left(\frac{e}{2 m}\right)^{2} \sum_{\mathbf{k}, \mathbf{p}} \int_{-\infty}^{\infty} \frac{d \varepsilon d \varepsilon^{\prime} d \omega^{\prime}}{(2 \pi)^{3} \omega^{\prime}}\left[h\left(\varepsilon^{\prime}+\omega^{\prime}\right)-h\left(\varepsilon^{\prime}\right)\right]\left\{\mathbf{k}\left[\mathbf{E}_{d r i}\left(\omega^{\prime}\right) \cdot \mathbf{k}\right] \times\right. \\
& \left.\left(\overline{\mathcal{G}}_{\mathbf{k}}^{R} \overline{\mathcal{G}}_{\mathbf{k}}^{A}\right)^{2} \mathrm{C}\left(\mathbf{p}, \mathbf{p} ; \omega+\omega^{\prime}-\varepsilon, \varepsilon, \omega^{\prime}-\omega\right)\right\} .
\end{aligned}
$$

The vector potential $\mathbf{A}_{d r i}$ was expressed through the electric field and $\overline{\mathcal{G}}_{\mathbf{k}}^{R / A}$ are abbreviations for ${\overline{\mathcal{G}^{0}}}_{\mathbf{k}}^{R / A}(\omega=0)$. Integration- and summation-variables where changed according to $\mathbf{k}+\mathbf{p} \rightarrow \mathbf{p}$ and $\varepsilon-\varepsilon^{\prime} \rightarrow \varepsilon$. Since only the spatially averaged current is considered and a spatially homogeneous (on average) system is presumed, $\mathbf{j}$ must be parallel to $\mathbf{E}_{d r i}$. Eq.(2.62) can then be written as

$$
\begin{aligned}
\delta \mathbf{j}_{W L}(\omega)= & -\frac{2}{\mathcal{V}^{2} d}\left(\frac{e}{2 m}\right)^{2} \sum_{\mathbf{k}, \mathbf{p}} \int_{-\infty}^{\infty} \frac{d \varepsilon d \varepsilon^{\prime} d \omega^{\prime}}{(2 \pi)^{3} \omega^{\prime}}\left[h\left(\varepsilon^{\prime}+\omega^{\prime}\right)-h\left(\varepsilon^{\prime}\right)\right]\left\{|\mathbf{k}|^{2} \mathbf{E}_{d r i}\left(\omega^{\prime}\right) \times\right. \\
& \left.\left(\overline{\mathcal{G}}_{\mathbf{k}}^{R} \overline{\mathcal{G}}_{\mathbf{k}}^{A}\right)^{2} \mathrm{C}\left(\mathbf{p}, \mathbf{p} ; \omega+\omega^{\prime}-\varepsilon, \varepsilon, \omega^{\prime}-\omega\right)\right\}
\end{aligned}
$$


The Green's functions yield dominant contributions for $|\mathbf{k}| \simeq k_{F}$ and therefore $|\mathbf{k}|^{2}$ can be replaced by $k_{F}^{2}$. Assuming a constant average level-spacing $\Delta$ in the vicinity of the Fermi energy, the k-sum can now be carried out:

$$
\sum_{\mathbf{k}} k_{F}^{2}\left({\overline{\mathcal{G}^{0}}}_{\mathbf{k}}^{R}{\overline{\mathcal{G}^{0}}}_{\mathbf{k}}^{A}\right)^{2} \simeq k_{F}^{2} \frac{4 \pi \tau_{e l}^{3}}{\Delta} .
$$

The $\varepsilon^{\prime}$-integration can be done as well,

$$
\int_{-\infty}^{\infty} d \varepsilon^{\prime}\left[h\left(\varepsilon^{\prime}+\omega^{\prime}\right)-h\left(\varepsilon^{\prime}\right)\right]=2 \omega^{\prime}
$$

Inserting these results into Eq.(2.63) yields

$$
\begin{array}{r}
\delta \mathbf{j}_{W L}(\omega)=-\frac{8 k_{F}^{2} \tau_{e l}^{3}}{\Delta L^{2 d} d}\left(\frac{e}{2 m}\right)^{2} \sum_{\mathbf{p}}^{\prime} \int_{-\infty}^{\infty} \frac{d \varepsilon d \omega^{\prime}}{(2 \pi)^{2}} \mathbf{E}_{d r i}\left(\omega^{\prime}\right) \times \\
\mathrm{C}\left(\mathbf{p}, \mathbf{p} ; \omega+\omega^{\prime}-\varepsilon, \varepsilon, \omega^{\prime}-\omega\right) .
\end{array}
$$

Transverse modes have again been integrated out, and the summation is restricted to the diffusive regime. Since the system is translational invariant after averaging over disorder, the Cooperon depends on the difference of its space-variables; $\mathrm{C}\left(\mathbf{r}, \mathbf{r}^{\prime}\right)=$ $\mathrm{C}\left(\mathbf{r}-\mathbf{r}^{\prime}\right)$; or equivalently in momentum space $\mathrm{C}\left(\mathbf{p}, \mathbf{p}^{\prime}\right)=L^{d} \delta_{\mathbf{p}, \mathbf{p}^{\prime}} \mathrm{C}(\mathbf{p})$. Fouriertransforming to space and time variables finally results in

$$
\delta \mathbf{j}_{W L}(\omega)=-\frac{2 e^{2} D}{\pi} \frac{\pi \tau_{e l}^{2}}{\Delta} \int_{-\infty}^{\infty} d t d t^{\prime} \mathbf{E}_{d r i}\left(t^{\prime}\right) \mathrm{C}\left(\mathbf{r}, \mathbf{r} ; t,-t,-t-t^{\prime}\right) e^{i \omega\left(2 t+t^{\prime}\right)} .
$$

Using the Einstein relation $\sigma_{d}=e^{2} \nu_{d} D$, the rescaled Cooperon $\mathcal{C}$ defined in Eq.(2.57) and substituting $2 t \rightarrow t$ one arrives at

$$
\delta \mathbf{j}_{W L}(\omega)=-\frac{\sigma_{d}}{\pi \nu_{d}} \int_{-\infty}^{\infty} d t d t^{\prime} \mathbf{E}_{d r i}\left(t^{\prime}\right) \mathcal{C}\left(\mathbf{r}, \mathbf{r} ; \frac{t}{2},-\frac{t}{2},-\left(\frac{t}{2}+t^{\prime}\right)\right) e^{i \omega\left(t+t^{\prime}\right)}
$$

This expression will be used to derive the dephasing time due to electron-electron interactions with small energy transfer in a non-perturbative way. To clarify the connection to standard perturbation theory in the two-particle Coulomb interaction and the role of the fluctuation-dissipation theorem, a calculation of $\tau_{\varphi}$ to first order in the Random-Phase-Approximation is given in the next section. This method strongly depends on the cutoff $\Gamma_{0}$ which makes the result a priori uncontrolled in the physically interesting limit $\Gamma_{0} \rightarrow 0$. 


\subsection{The dephasing time to first order in the screened interaction}

The system under consideration is an ensemble of interacting, spinless electrons in a static disorder potential and a spatially constant external driving field $\mathbf{E}_{d r i}(t)=$ $-(1 / c) \partial_{t} \mathbf{A}_{d r i}(t)$. The Hamiltonian describing this system is

$$
\hat{H}=\hat{H}_{0}+\hat{H}_{1}(t)+\hat{H}_{2},
$$

where $\hat{H}_{0}$ and $\hat{H}_{1}$ are given in Eq.(2.10) and Eq.(2.14). The interaction is described by

$$
\hat{H}_{2}=\frac{1}{2} \int d \mathbf{r} d \mathbf{r}^{\prime} f\left(\mathbf{r}-\mathbf{r}^{\prime}\right) \hat{\Psi}^{\dagger}(\mathbf{r}) \hat{\Psi}^{\dagger}\left(\mathbf{r}^{\prime}\right) \hat{\Psi}\left(\mathbf{r}^{\prime}\right) \hat{\Psi}(\mathbf{r}) .
$$

$f(\mathbf{r})$ is the bare Coulomb potential

$$
f(\mathbf{r})=\frac{e^{2}}{|\mathbf{r}|} .
$$

To derive interaction corrections to the conductivity, it is convenient to use the equilibrium Matsubara technique. This can be done, since conductivity is a linear response function and the fluctuation-dissipation theorem gives the connection between the imaginary- and real-time quantities. In Appendix A it is shown, that for non-interacting electrons an equivalent relation exists for response functions of arbitrary order. Here the notations of App. A will be used. The imaginary time paramagnetic current is defined in Eq. A.24). The auxiliary field will, relating to the potential $\mathbf{A}_{d r i}$, now be named $\tilde{\mathbf{A}}_{d r i}$ and it satisfies the boundary condition $\tilde{\mathbf{A}}_{d r i}(\tau)=\tilde{\mathbf{A}}_{d r i}(\tau-n \beta)$ for any integer $n$. The time-evolution operator $\hat{U}$ describes the evolution due to $\tilde{\mathbf{A}}_{d r i}(\tau)$ and the interaction $\hat{H}_{2}$ :

$$
\hat{U}(\beta, 0)=\hat{T} \exp \left[-\int_{0}^{\beta} d \tau^{\prime}\left[\hat{H}_{1, D}\left(\tau^{\prime}\right)+\hat{H}_{2, D}\left(\tau^{\prime}\right)\right]\right] .
$$

The first order (in the auxiliary field) contribution to the spatially averaged current $\mathbf{j}(\tau)=\mathbf{j}(\mathbf{Q}=\mathbf{0}, \tau)$ is

$$
\delta \mathbf{j}_{\text {para }}(\tau)=\frac{e^{2}}{2 m^{2} \mathcal{V} c d} \int_{0}^{\beta} d \tau^{\prime} \tilde{\mathbf{A}}_{d r i}\left(\tau^{\prime}\right) \sum_{\mathbf{k}, \mathbf{q}} \mathbf{q} \cdot \mathbf{k}\left\langle\hat{T} \tilde{U}(\beta, 0) \hat{\Psi}_{\mathbf{k}}^{\dagger}\left(\tau^{\prime}\right) \hat{\Psi}_{\mathbf{k}}\left(\tau^{\prime}\right) \hat{\Psi}_{\mathbf{q}}^{\dagger}(\tau) \hat{\Psi}_{\mathbf{q}}(\tau)\right\rangle_{0}^{c o n}
$$

The reduced time-evolution operator $\tilde{U}$ describes the evolution due to the electronelectron interaction,

$$
\tilde{U}(\beta, 0)=\hat{T} \exp \left[-\int_{0}^{\beta} d \tau^{\prime} \hat{H}_{2, D}\left(\tau^{\prime}\right)\right]
$$


When calculating the correlator in Eq.(2.73), only totally connected diagrams must be kept, as indicated by the index con. The expectation value $\langle\cdots\rangle_{0}=$ $\left(1 / Z_{0}\right) \operatorname{Sp}\left[e^{-\beta \hat{H}_{0}} \cdots\right]$ is taken with respect to the non-interacting Hamiltonian $\hat{H}_{0}$ and the system is assumed to be spatially homogeneous on average. The perturbation theory in the interaction is done using the standard diagrammatic technique [11]. To take screening of the bare Coulomb interaction into account, the expansion is done in the Random-Phase-Approximation potential $f_{\mathrm{RPA}}(\mathbf{q}, i \omega)$ instead of the (Fourier transformed of the) bare interaction $f_{\mathbf{q}}$. Diagrammatically the RPA-interaction is depicted in Fig.2.6. In the disordered system considered here, the electronic motion is diffusive. Consequently the density-density correlation function has a diffusion pole, which in the diagrammatic language is produced by the Diffuson $\mathrm{D}_{0}$ in the polarization-bubble. The Dyson equation for the Diffuson is shown in Fig.2.6(b). It is obtained from the Cooperon by reversing one electron line. Since particle-number conservation always requires a diffusive pole in the density-density correlation function, independent of the interactions in the system, no cutoff similar to $1 / \tau_{\varphi}$ enters the Diffuson [13]. $\mathrm{D}_{0}$, as it is introduced here, describes a classical diffusion process and therefore a quantum mechanical quantity like the dephasing time $\tau_{\varphi}$ does not appear. (In different context, e.g. in calculating Universal Conductance Fluctuations, 'Diffusons' appear as formal tools in diagrammatic perturbation theory and are not directly related to a physical diffusion process. Such 'Diffusons' are indeed affected by dephasing effects as will be discussed in Sec.(3.5)). In the model of disorder considered here, the current-current correlation function that defines the WL-correction to the spatially averaged $(\mathbf{Q}=\mathbf{0}$ mode) conductivity is, for symmetry reasons, not renormalized by Diffusons. This is due to the $\delta$-potential approximation for the disorder scattering centers which only give rise to $s$-wave scattering. In this case the momentum relaxation time $\tau_{e l}$ is equal to the transport time entering the Drude conductivity. For extended scatterers, where contributions from higher angular momenta become important, Diffuson corrections are needed in the current-current correlation function to obtain the correct transport time [21]. In the Matsubara formulation one obtains the following expressions for the disorder-averaged Green's function (see Eq.(2.43)) and the Diffuson $\mathrm{D}_{0}$ :

$$
\begin{gathered}
\overline{\mathcal{G}}_{\mathbf{k}}(i \omega)=\frac{1}{i \omega-\left(\frac{\mathbf{k}^{2}}{2 m}-\mu\right)+\frac{i}{2 \tau_{e l}} \operatorname{sgn}(\omega)}, \\
\mathrm{D}_{0}(\mathbf{Q}, i \omega)=\frac{\Delta}{2 \pi \tau_{e l}^{2}} \frac{1}{D \mathbf{Q}^{2}+|\omega|} .
\end{gathered}
$$

Analytically the diagrams in Fig.2.6 can be summed in a geometric series, yielding

$$
f_{\mathbf{q}}^{\mathrm{RPA}}(i \omega)=\frac{f_{\mathbf{q}}}{1-f_{\mathbf{q}} \overline{\Pi_{0}}(\mathbf{q}, i \omega)}=: \frac{f_{\mathbf{q}}}{\epsilon(\mathbf{q}, i \omega)}
$$




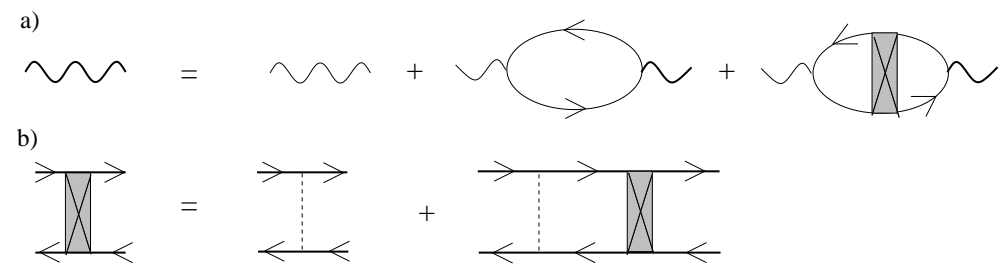

Figure 2.6: Diagrammatic definition of the effective RPA interaction. Thick wiggled lines represent $f_{\mathrm{RPA}}(\mathbf{q}, i \omega)$ while thin wiggled ones stand for the bare interaction $f_{\mathbf{q}}$. The electron-lines here are the disorder averaged Green's functions $\overline{\mathcal{G}^{0}}$ defined in Eq.(2.75). The crossed, shaded box is the Diffuson $D_{0}$. Disorder scattering is symbolized by dashed lines.

where the dielectric function $\epsilon(\mathbf{q}, i \omega)$ was introduced. The irreducible polarizationfunction $\overline{\Pi_{0}}(\mathbf{q}, i \omega)$ is given by the sum of the two polarization-bubbles in Fig.2.6. In the low frequency $\left(\omega \ll 1 / \tau_{e l}\right)$, long wavelength $(|\mathbf{q}|<1 / l)$ limit one obtains

$$
\overline{\Pi_{0}}(\mathbf{q}, i \omega)=-\nu_{d} \frac{D \mathbf{q}^{2}}{D \mathbf{q}^{2}+|\omega|} .
$$

$f_{\mathbf{q}}$ is the Fourier-transformed of the bare interaction $f(\mathbf{r})$ and depends on the dimension of the system. In $d=3$ it is given by

$$
f_{\mathbf{q}}=\frac{4 \pi e^{2}}{\mathbf{q}^{2}} \text { for } d=3
$$

In lower dimensions one gets [20]

$$
\begin{gathered}
f_{\mathbf{q}}=\frac{2 \pi e^{2}}{|\mathbf{q}|} \text { for } d=2, \\
f_{\mathbf{q}}=e^{2} \ln \left(\frac{1}{\mathbf{q}^{2} a^{2}}\right) \text { for } d=1,
\end{gathered}
$$

where $a$ is the samples transverse extension and the one dimensional result is valid for $q<1 / a$. From Eq.(2.78) one obtains for the dielectric function in the diffusive regime

$$
\epsilon(\mathbf{q}, \omega)=1+f_{\mathbf{q}} \nu_{d} \frac{D \mathbf{q}^{2}}{D \mathbf{q}^{2}-i \omega}
$$

Figure 2.7 shows all diagrams that are obtained by expanding the current in Eq.(2.73) to first order in $f^{\mathrm{RPA}}$. Diagrams $\left.a\right)-c$ ) are Fock-type and $\left.d\right)-f$ ) are Hartree-type contributions. Even though $f^{\text {RPA }}$ is a disorder-averaged quantity, the electron-lines $\mathcal{G}_{0}$ are still non-averaged propagators, depending on the special realization of the random potential $U$. The non-trivial problem now is to perform the 

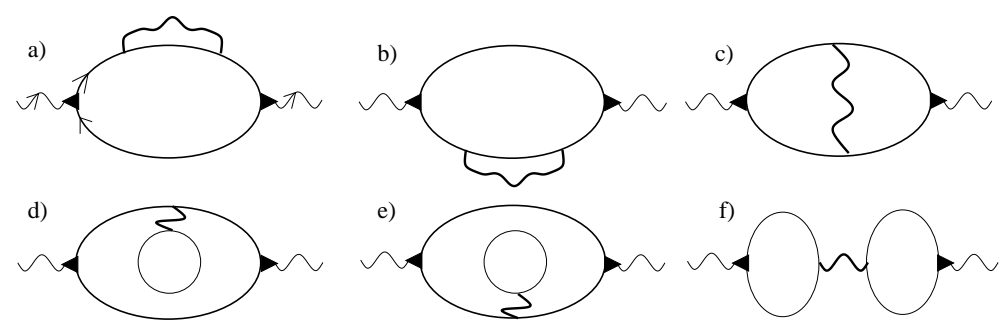

Figure 2.7: All diagrams for the current to first order in $f^{\mathrm{RPA}}$. Thin wiggled lines denote current-vertices and thick ones the effective interaction $f^{\mathrm{RPA}}$. The electronlines are, in contrast to Fig.2.0, the non-averaged Green's functions $\mathcal{G}_{0}$ that still contain the disorder exactly.

disorder average of the diagrams in Fig.2.7. Explicit calculations have first been done by Altshuler et al. in Ref. [20]. In the following only the main results will be briefly summarized as far as they are needed in the context of this work. The main focus is to show the connection between dephasing due to Nyquist noise, as discussed in section (3.3) and the diagrammatic perturbation theory in the screened interaction. Regarding the Hartree (or direct) contributions, it was found in Ref. [20], that after averaging all diagrams of type $d$ ) and $e$ ) that have disorder-lines (e.g. Cooperons) connecting the upper and lower electron-line of the bubble, cancel with the disorder average of diagram $f$ ). In other words, only diagrams where both electron propagators are averaged separately give non-vanishing Hartree contributions. This means, that the direct interaction has no effect on the correlations between time-reversed trajectories (or the upper and lower electron-line in the diagrammatic language) and can therefore not contribute to dephasing. The components entering the Hartree diagrams are the averaged non-interacting single particle Green's function $\overline{\mathcal{G}^{0}}$ and the disorder average of the single-bubble diagram shown in Fig.2.8. The same diagrams

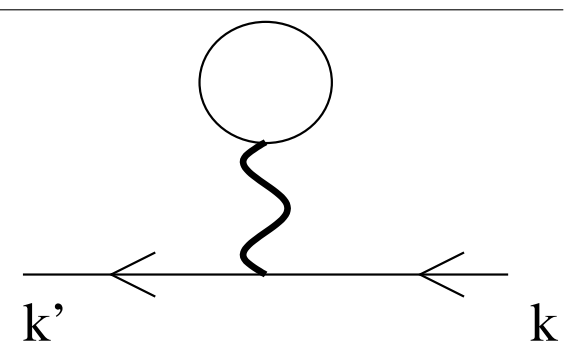

Figure 2.8: Electron-propagator that enters the disorder averaged Hartree contribution to the current. Averaging is indicated by the over-bar.

occur in the calculation of the average one-electron density of states

$$
\nu_{d}(\omega)=-\frac{2}{\pi \mathcal{V}} \sum_{\mathbf{q}} \operatorname{Im} \overline{\mathcal{G}}_{\mathbf{q}}(i \omega)_{i \omega \rightarrow \omega+i 0} .
$$


$\overline{\mathcal{G}}$ is the disorder averaged total Green's function including electron-electron interactions. The conductivity is related to the density of states at the Fermi energy $\left(\nu_{d}:=\nu_{d}\left(\varepsilon_{F}\right)\right)$ via the Einstein relation $\sigma_{d}=e^{2} \nu_{d} D$. The only effect of the Hartree interaction on the conductivity thus comes form a renormalization of $\nu_{d}$. In conclusion the diagrams $d)-f$ ) of Fig.2.7 do not contribute to dephasing. They can therefore be neglected in the following. From Eq.(2.73) and the diagrams $a)-d$ ) one easily finds the Fock contribution to the conductivity:

$$
\begin{aligned}
& \sigma_{\text {para }}^{\text {Fock }}(\varepsilon)=\frac{e^{2}}{i \varepsilon m^{2} \mathcal{V}^{2} d} \sum_{\mathbf{k}, \mathbf{q}} \sum_{\mathbf{p}, \mathbf{p}^{\prime}} \mathbf{k} \cdot \mathbf{q} \frac{1}{\beta^{2}}\left\{\sum_{\omega_{1}, \omega_{2}}[\right. \\
& f_{\mathbf{p}-\mathbf{p}^{\prime}}^{\mathrm{RPA}}\left(i \omega_{2}-i \omega_{1}\right) \mathcal{G}_{\mathbf{q}, \mathbf{p}}\left(i \tilde{\varepsilon}+i \omega_{2}\right) \mathcal{G}_{\mathbf{k}, \mathbf{p}^{\prime}}\left(i \omega_{1}\right) \mathcal{G}_{\mathbf{p}^{\prime}, \mathbf{q}}\left(i \omega_{2}\right) \mathcal{G}_{\mathbf{p}, \mathbf{k}}\left(i \tilde{\varepsilon}+i \omega_{1}\right) \\
& +f_{\mathbf{p}-\mathbf{p}^{\prime}}^{\mathrm{RPA}}\left(i \omega_{1}-i \omega_{2}\right) \mathcal{G}_{\mathbf{q}, \mathbf{p}}\left(i \omega_{1}\right) \mathcal{G}_{\mathbf{p}, \mathbf{p}^{\prime}}\left(i \omega_{2}\right) \mathcal{G}_{\mathbf{p}^{\prime}, \mathbf{k}}\left(i \omega_{1}\right) \mathcal{G}_{\mathbf{k}, \mathbf{q}}\left(i \omega_{1}-i \tilde{\varepsilon}\right) \\
& \left.\left.+f_{\mathbf{p}-\mathbf{p}^{\prime}}^{\mathrm{RPA}}\left(i \omega_{1}-i \omega_{2}\right) \mathcal{G}_{\mathbf{q}, \mathbf{p}}\left(i \omega_{1}\right) \mathcal{G}_{\mathbf{p}, \mathbf{p}^{\prime}}\left(i \omega_{2}\right) \mathcal{G}_{\mathbf{p}^{\prime}, \mathbf{k}}\left(i \omega_{1}\right) \mathcal{G}_{\mathbf{k}, \mathbf{q}}\left(i \omega_{1}+i \tilde{\varepsilon}\right)\right]\right\}_{i \tilde{\varepsilon} \rightarrow \varepsilon+i 0} .
\end{aligned}
$$

$i \tilde{\varepsilon}$ is a Bosonic Matsubara frequency, all other ones are Fermionic. The Matsubara Green's function $\mathcal{G}(i \omega)$ coincides with the analytic continuation of the retarded function $\mathcal{G}^{R}(i \omega)$ for positive $\omega$ and with $\mathcal{G}^{A}(i \omega)$ for $\omega<0$ (see Eqs. A.35), (A.36) which are also valid for $\mathcal{G}(i \omega))$. Thus the frequency sums in Eq.(2.84) produce various different products of retarded and advanced functions which have to be averaged over disorder separately. This very technical procedure, which affords much experience in diagrammatic perturbation theory, is described in detail by Aleiner et al. in Ref. [32]. Also a physical interpretation of the different terms occuring is given in that article. However, if one is only interested in pure dephasing effects, the problem greatly simplifies. In this case only interactions with small frequencytransfer $\varepsilon \sim 1 / \tau_{\varphi}$, which are so weak that the classical motion of the electrons remains unchanged, are considered. Such nearly elastic scattering thus only effects the electrons phase and is therefore called a "pure" dephasing effect here. Of course inelastic scattering with energy-transfer $\varepsilon \gg 1 / \tau_{\varphi}$, besides its impact on the density of states and the electron dynamics, also destroys the phase-information. This separation into 'dephasing' and 'interaction' contributions is analogous to what is done in deriving Eq.(2.67) for the current in the presence of a fluctuating external field. There also only dephasing effects are explicitly treated, and the cutoff $\Gamma_{0}$ is introduced to take account of electron-electron interactions with large energy transfer as well as all other possible sources of dephasing. It is therefore assumed, that the field-vertices do not change the analytic structure of the Green's functions. After each interaction retarded propagators remain retarded and advanced ones stay advanced. In order to describe such dephasing effects due to the intrinsic electron 
electron interaction, thus only the following terms have to be kept:

$$
\begin{aligned}
& \sigma_{\text {para }}^{\text {deph }}(\varepsilon)=\frac{e^{2}}{i \varepsilon m^{2} \mathcal{V}^{2} d} \sum_{\mathbf{k}, \mathbf{q}} \sum_{\mathbf{p}, \mathbf{p}^{\prime}} \mathbf{k} \cdot \mathbf{q} \frac{1}{\beta^{2}}[ \\
& \sum_{\left(-\tilde{\varepsilon}<\omega_{1}<0\right)} \sum_{\left(-\tilde{\varepsilon}<\omega_{2}<0\right)} f_{\mathbf{p}-\mathbf{p}^{\prime}}^{\mathrm{RPA}}\left(i \omega_{2}-i \omega_{1}\right) \mathcal{G}_{\mathbf{q}, \mathbf{p}}\left(i \tilde{\varepsilon}+i \omega_{2}\right) \mathcal{G}_{\mathbf{k}, \mathbf{p}^{\prime}}\left(i \omega_{1}\right) \mathcal{G}_{\mathbf{p}^{\prime}, \mathbf{q}}\left(i \omega_{2}\right) \mathcal{G}_{\mathbf{p}, \mathbf{k}}\left(i \tilde{\varepsilon}+i \omega_{1}\right) \\
& +\sum_{\left(0<\omega_{1}<\tilde{\varepsilon}\right)} \sum_{\left(0<\omega_{2}<\tilde{\varepsilon}\right)} f_{\mathbf{p}-\mathbf{p}^{\prime}}^{\mathrm{RPA}}\left(i \omega_{1}-i \omega_{2}\right) \mathcal{G}_{\mathbf{q}, \mathbf{p}}\left(i \omega_{1}\right) \mathcal{G}_{\mathbf{p}, \mathbf{p}^{\prime}}\left(i \omega_{2}\right) \mathcal{G}_{\mathbf{p}^{\prime}, \mathbf{k}}\left(i \omega_{1}\right) \mathcal{G}_{\mathbf{k}, \mathbf{q}}\left(i \omega_{1}-i \tilde{\varepsilon}\right) \\
& \left.+\sum_{\left(-\tilde{\varepsilon}<\omega_{1}<0\right)} \sum_{\left(-\tilde{\varepsilon}<\omega_{2}<0\right)} f_{\mathbf{p}-\mathbf{p}^{\prime}}^{\mathrm{RPA}}\left(i \omega_{1}-i \omega_{2}\right) \mathcal{G}_{\mathbf{q}, \mathbf{p}}\left(i \omega_{1}\right) \mathcal{G}_{\mathbf{p}, \mathbf{p}^{\prime}}\left(i \omega_{2}\right) \mathcal{G}_{\mathbf{p}^{\prime}, \mathbf{k}}\left(i \omega_{1}\right) \mathcal{G}_{\mathbf{k}, \mathbf{q}}\left(i \omega_{1}+i \tilde{\varepsilon}\right)\right]_{i \tilde{\varepsilon} \rightarrow \varepsilon+i 0}
\end{aligned}
$$

The frequencies are limited so that all electron-lines on one side of the bubble ("upper" or "lower") are either all retarded or all advanced. The analytic structure of the Green's functions does not change over the range of summation, but that of $f^{\mathrm{RPA}}$ does, depending on whether $\omega_{1}$ is smaller or greater than $\omega_{2}$. The frequency sums can be evaluated by contour-integration. After a straightforward calculation one obtains

$$
\begin{aligned}
& \sigma_{\text {para }}^{\text {deph }}(\varepsilon)=\frac{e^{2}}{i \varepsilon m^{2} \mathcal{V}^{2} d} \sum_{\mathbf{k}, \mathbf{q}} \sum_{\mathbf{p}, \mathbf{p}^{\prime}} \mathbf{k} \cdot \mathbf{q} \int_{-\infty}^{\infty} d \omega d \omega^{\prime}\left\{\left[f\left(\omega^{\prime}\right)-f\left(\omega^{\prime}-\varepsilon\right)\right] \times\right. \\
& {\left[f\left(\omega+\omega^{\prime}\right) f_{\mathbf{p}-\mathbf{p}^{\prime}}^{\mathrm{RPA}, A}(\omega)-f\left(\omega+\omega^{\prime}-\varepsilon\right) f_{\mathbf{p}-\mathbf{p}^{\prime}}^{\mathrm{RPA}, R}(\omega)\right]+n(\omega)\left[f\left(\omega^{\prime}\right)-f\left(\omega^{\prime}-\varepsilon\right)\right] \times } \\
& {\left.\left[f_{\mathbf{p}-\mathbf{p}^{\prime}}^{\mathrm{RPA}}(\omega)-f_{\mathbf{p}-\mathbf{p}^{\prime}}^{\mathrm{RPA}, R}(\omega)\right]\right\}\left\{\mathcal{G}_{\mathbf{q}, \mathbf{p}}^{R}\left(\omega+\omega^{\prime}\right) \mathcal{G}_{\mathbf{k}, \mathbf{p}^{\prime}}^{A}\left(\omega^{\prime}-\varepsilon\right) \mathcal{G}_{\mathbf{p}^{\prime}, \mathbf{q}}^{A}\left(\omega+\omega^{\prime}-\varepsilon\right) \mathcal{G}_{\mathbf{p}, \mathbf{k}}^{R}(\omega)\right.} \\
+ & \mathcal{G}_{\mathbf{q}, \mathbf{p}}^{R}\left(\omega^{\prime}\right) \mathcal{G}_{\mathbf{p}, \mathbf{p}^{\prime}}^{R}\left(\omega+\omega^{\prime}\right) \mathcal{G}_{\mathbf{p}^{\prime}, \mathbf{k}}^{R}\left(\omega^{\prime}\right) \mathcal{G}_{\mathbf{k}, \mathbf{q}}^{A}\left(\omega^{\prime}-\varepsilon\right) \\
+ & \left.\mathcal{G}_{\mathbf{q}, \mathbf{p}}^{A}\left(\omega^{\prime}-\varepsilon\right) \mathcal{G}_{\mathbf{p}, \mathbf{p}^{\prime}}^{A}\left(\omega+\omega^{\prime}-\varepsilon\right) \mathcal{G}_{\mathbf{p}^{\prime}, \mathbf{k}}^{A}\left(\omega^{\prime}-\varepsilon\right) \mathcal{G}_{\mathbf{k}, \mathbf{q}}^{R}\left(\omega^{\prime}\right)\right\} .
\end{aligned}
$$

The retarded and advanced RPA-propagators are obtained form the corresponding Matsubara expression by the usual continuations, $f^{\mathrm{RPA}, R / A}(\omega)=f^{\mathrm{RPA}}(i \tilde{\omega} \rightarrow$ $\omega \pm i 0)$. The complex function $f^{\mathrm{RPA}}(z)$ has a branch cut along the real axis. $n(\omega)=1 /(\exp (\beta \omega)-1)$ is the Bose function and $f(\omega)=1 /(\exp (\beta \omega)+1)$ the Fermi function. Eq. 2.86) now has to be averaged over disorder. The dominant contributions according to Ref. [32] are shown in Fig.2.9.

These crossed Cooperon diagrams generate a maximal number of Cooperon poles. Evaluating this diagrams gives the leading dephasing contribution to the weak lo- 

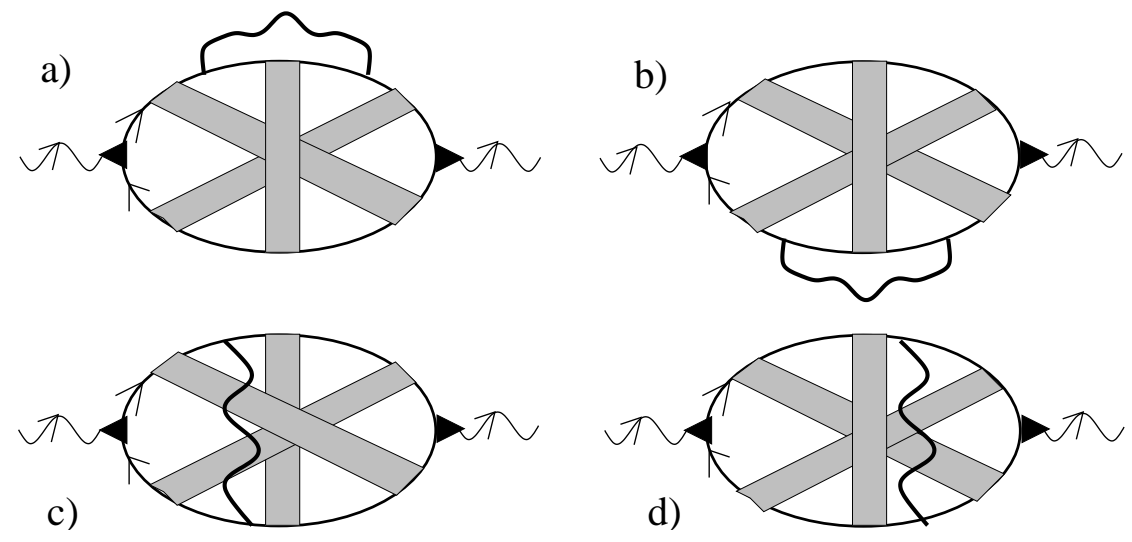

Figure 2.9: Diagrams contributing to $\sigma_{W L}$ in first order in the screened interaction. Shaded boxes represent the non-interacting Cooperon $\mathcal{C}_{0}$. Filled triangles indicate current-vertices and the RPA-propagator is symbolized by thick wiggled lines. The upper electron line is retarded, the lower advanced. Diagrams (c) and (d) are vertex corrections.

calization correction:

$$
\begin{aligned}
& \sigma_{\text {para }}^{\text {deph }}(\varepsilon)=\frac{e^{2} D}{2 \pi^{2} i \varepsilon \mathcal{V}^{2}} \sum_{\mathbf{k}, \mathbf{q}} \int_{-\infty}^{\infty} d \omega\left\{[\varepsilon+\omega][n(\varepsilon+\omega)-n(\omega)] f_{\mathbf{k}}^{\mathrm{RPA}, A}(\omega)\right. \\
& \left.-[\varepsilon-\omega][n(\omega-\varepsilon)-n(\omega)] f_{\mathbf{k}}^{\mathrm{RPA}, R}(\omega)\right\}\left\{2 \mathcal{C}_{0}(\mathbf{q}, \varepsilon+\omega) \mathcal{C}_{0}(\mathbf{q}, \varepsilon-\omega) \mathcal{C}_{0}(\mathbf{q}-\mathbf{k}, \varepsilon)\right. \\
& \left.-\mathcal{C}_{0}^{2}(\mathbf{q}, \varepsilon) \mathcal{C}_{0}(\mathbf{q}-\mathbf{k}, \varepsilon+\omega)-\mathcal{C}_{0}^{2}(\mathbf{q}, \varepsilon) \mathcal{C}_{0}(\mathbf{q}-\mathbf{k}, \varepsilon-\omega)\right\}
\end{aligned}
$$

This is the first order RPA correction to the WL-result due to electron-electron interactions with small frequency transfer. The Bose functions restrict the range of integration to frequencies $\omega \leq \max (T, \varepsilon)$. In this regime the effect of the Coulomb interaction can be described by a fluctuating random field, so called Nyquist noise, which is produced by the motion of the electrons in the system. This will be described in detail in the next chapter. The contribution to dephasing from the frequency regime $\omega \leq \max (T, \varepsilon)$ is therefore called Nyquist noise contribution and denoted by $1 / \tau_{n n}$. Since different dephasing mechanisms are assumed to be independent, the total dephasing rate is $1 / \tau_{\varphi}=\Gamma_{0}+1 / \tau_{n n}$ and $\Gamma_{0}$ contains all dephasing effects other than Nyquist noise. If $\Gamma_{0} \gg 1 / \tau_{n n}$, which can be achieved by applying an external magnetic field, Eq.(2.32) can be expanded in powers of $1 /\left(\Gamma_{0} \tau_{n n}\right)$. To first order this yields

$$
\sigma_{W L}(\varepsilon)=-\frac{\sigma_{d}}{\pi \nu_{d}} \frac{1}{\mathcal{V}} \sum_{|\mathbf{q}|<1 / l}\left[\mathcal{C}_{0}(\mathbf{q}, \varepsilon)-\frac{1}{\tau_{n n}} \mathcal{C}_{0}^{2}(\mathbf{q}, \varepsilon)\right]
$$

where $\mathcal{C}_{0}$ is defined in Eq.(2.60). To extract $1 / \tau_{n n}$ from Eq.(2.87), this equation is 
rewritten as

$$
\begin{aligned}
& \sigma_{\text {para }}^{\text {deph }}(\varepsilon)=\frac{e^{2} D}{2 \pi^{2} i \varepsilon \mathcal{V}^{2}} \sum_{\mathbf{q}} \mathcal{C}_{0}(\mathbf{q}, \varepsilon) \sum_{\mathbf{k}} \int_{-\infty}^{\infty} d \omega\left\{[\varepsilon+\omega][n(\varepsilon+\omega)-n(\omega)] f_{\mathbf{k}}^{\mathrm{RPA}, A}(\omega)\right. \\
& \left.-[\varepsilon-\omega][n(\omega-\varepsilon)-n(\omega)] f_{\mathbf{k}}^{\mathrm{RPA}, R}(\omega)\right\}\left\{2 \mathcal{C}_{0}(\mathbf{q}+\mathbf{k}, \varepsilon+\omega) \mathcal{C}_{0}(\mathbf{q}+\mathbf{k}, \varepsilon-\omega) \mathcal{C}_{0}^{-1}(\mathbf{q}, \varepsilon)\right. \\
& \left.-\mathcal{C}_{0}(\mathbf{q}-\mathbf{k}, \varepsilon+\omega)-\mathcal{C}_{0}(\mathbf{q}-\mathbf{k}, \varepsilon-\omega)\right\} .
\end{aligned}
$$

Using $\sigma_{d} / \nu_{d}=e^{2} D$ and comparing Eq.(2.88) and Eq.(2.89) one obtains the following expression for $1 / \tau_{n n}$ to first order RPA:

$$
\begin{aligned}
& \frac{1}{\tau_{n n}(\varepsilon)}=\lim _{\mathbf{q} \rightarrow \mathbf{0}} \frac{1}{2 \pi i \varepsilon \mathcal{V}} \sum_{\mathbf{k}} \int_{-\infty}^{\infty} d \omega\left\{[\varepsilon+\omega][n(\varepsilon+\omega)-n(\omega)] f_{\mathbf{k}}^{\mathrm{RPA}, A}(\omega)\right. \\
& \left.-[\varepsilon-\omega][n(\omega-\varepsilon)-n(\omega)] f_{\mathbf{k}}^{\mathrm{RPA}, R}(\omega)\right\}\left\{2 \mathcal{C}_{0}(\mathbf{q}+\mathbf{k}, \varepsilon+\omega) \mathcal{C}_{0}(\mathbf{q}+\mathbf{k}, \varepsilon-\omega) \mathcal{C}_{0}^{-1}(\mathbf{q}, \varepsilon)\right. \\
& \left.-\mathcal{C}_{0}(\mathbf{q}-\mathbf{k}, \varepsilon+\omega)-\mathcal{C}_{0}(\mathbf{q}-\mathbf{k}, \varepsilon-\omega)\right\} .
\end{aligned}
$$

Inserting the explicit form of $\mathcal{C}_{0}$, this equation can be reduced to

$$
\begin{aligned}
\frac{1}{\tau_{n n}(\varepsilon)}=-\frac{1}{\pi i \varepsilon \mathcal{V}} \sum_{\mathbf{k}} \int_{-\infty}^{\infty} & d \omega\left\{[\varepsilon+\omega][n(\varepsilon+\omega)-n(\omega)] f_{\mathbf{k}}^{\mathrm{RPA}, A}(\omega)\right. \\
& \left.-[\varepsilon-\omega][n(\omega-\varepsilon)-n(\omega)] f_{\mathbf{k}}^{\mathrm{RPA}, R}(\omega)\right\} \times \\
& \frac{D \mathbf{k}^{2}}{\left(D \mathbf{k}^{2}-i \varepsilon-i \omega+\Gamma_{0}\right)\left(D \mathbf{k}^{2}-i \varepsilon+i \omega+\Gamma_{0}\right)}
\end{aligned}
$$

Eq.(2.91) can be solved in the limiting cases $T \gg \varepsilon$ and $T \ll \varepsilon$. For $T \gg \varepsilon$ the function $n(\omega \pm \varepsilon)$ is expanded to first order in $\varepsilon$. The derivative of the Bose function is

$$
\frac{d n}{d \omega}=-\frac{\beta}{4 \sinh ^{2}\left(\frac{\beta \omega}{2}\right)} .
$$

To evaluate the $\omega$-integral, the approximation

$$
\frac{\beta}{\sinh ^{2}\left(\frac{\beta \omega}{2}\right)} \approx \frac{4}{\beta \omega^{2}} \Theta\left(|\omega|-\frac{1}{\beta}\right)
$$

is made. Here $\Theta(x)$ is the usual step function. This yields $(1 / \beta=T)$

$$
\frac{1}{\tau_{n n}(T)}=\frac{2 T}{\pi \mathcal{V}} \sum_{\mathbf{k}} \int_{-T}^{T} d \omega \frac{\operatorname{Im}\left[f_{\mathbf{k}}^{\mathrm{RPA}, A}(\omega)\right]}{\omega} \frac{D \mathbf{k}^{2}}{\left(D \mathbf{k}^{2}-i \omega+\Gamma_{0}\right)\left(D \mathbf{k}^{2}+i \omega+\Gamma_{0}\right)} .
$$

In metals, where the screening is strong and the electrons motion is diffusive the essential contribution comes from momenta $|\mathbf{k}| \sim \sqrt{\varepsilon / D}$ with $\varepsilon \ll 1 / \tau_{e l}$. One can then approximate

$$
f_{\mathbf{k}}^{\mathrm{RPA}, A}(\omega) \approx \frac{D \mathbf{k}^{2}+i \omega}{\nu_{d} D \mathbf{k}^{2}}
$$


and consequently

$$
\frac{1}{\tau_{n n}(T)}=\frac{2 T}{\pi \nu_{d} \mathcal{V}} \sum_{\mathbf{k}} \int_{-T}^{T} d \omega \frac{1}{\left(D \mathbf{k}^{2}-i \omega+\Gamma_{0}\right)\left(D \mathbf{k}^{2}+i \omega+\Gamma_{0}\right)}
$$

For macroscopic samples with continuous spectrum the above expression can be calculated by replacing $(1 / \mathcal{V}) \sum_{\mathbf{k}} \rightarrow \int d \mathbf{k} /(2 \pi)^{d}$. In the limit $T \gg \Gamma_{0}$ one obtains

$$
\frac{1}{\tau_{n n}(T)} \propto \begin{cases}\frac{T}{\nu_{1} \sqrt{D \Gamma_{0}}} & \text { for } d=1 \\ \frac{T}{2 \pi \nu_{2} D} \ln \left(\frac{T}{\Gamma_{0}}\right) & \text { for } d=2 \\ \frac{T^{3 / 2}}{\sqrt{2} \pi^{2} \nu_{3} D^{3 / 2}} & \text { for } d=3 .\end{cases}
$$

A priori these results are only valid if $\Gamma_{0} \gg 1 / \tau_{n n}$.

Eq.(2.91) can also be used to obtain the frequency-dependence of $1 / \tau_{n n}$ in the limit $\varepsilon \gg T$ and $\varepsilon \gg \Gamma_{0}$. In this case Eq.(2.91) reduces to

$$
\frac{1}{\tau_{n n}(\varepsilon)}=-\frac{2}{\pi i \varepsilon \mathcal{V}} \operatorname{Re} \sum_{\mathbf{k}} \int_{-\varepsilon}^{0} d \omega \frac{(\varepsilon+\omega) f_{\mathbf{k}}^{\mathrm{RPA}, A}(\omega) D \mathbf{k}^{2}}{\left(D \mathbf{k}^{2}-i \varepsilon-i \omega\right)\left(D \mathbf{k}^{2}-i \varepsilon+i \omega\right)} .
$$

Here the real part was taken, since only this quantity can be physically interpreted as a damping-time. Solving the integrals for macroscopic systems yields

$$
\frac{1}{\tau_{n n}(\varepsilon)} \propto \frac{1}{\nu_{d}}\left(\frac{\varepsilon}{D}\right)^{\frac{d}{2}}
$$

Now the condition $\Gamma_{0} \gg 1 / \tau_{n n}$ for the validity of the first order expansion is replaced by $\varepsilon \gg 1 / \tau_{n n}$. Using $1 / \nu_{d}=\Delta L^{d}, E_{c}=D / L^{2}$ and Eq.(2.99) this means $\varepsilon^{1-d / 2} \gg$ $\Delta / E_{c}^{d / 2}$. Since $E_{c} \gg \Delta$ this condition is always satisfied in $d=2$. For $d=1$ one obtains the restriction $\varepsilon \gg \Delta\left(\Delta / E_{c}\right)$ and in three dimensions $\varepsilon \ll E_{c}\left(E_{c} / \Delta\right)^{2}$. In the next chapter the above results will be reproduced by means of a non-perturbative Eikonal ansatz. 


\section{Chapter 3}

\section{Eikonal approach to dephasing in disordered conductors}

\subsection{Introduction}

Parts of the content of this chapter have already been published in Ref. [1]. In addition to details of the calculations new results for the the frequency dependence of $1 / \tau_{\varphi}$ are presented. Furthermore the method derived in [1] is applied to study the Diffuson in the presence of external fields.

In a seminal paper, Altshuler, Aronov and Khmelnitskii [22, 8] developed an elegant, semiclassical method for calculating the dephasing rate due to Nyquist noise $1 / \tau_{n n}$ in disordered conductors at low temperatures. Using the equivalence between electron-electron interactions with small energy transfers and fluctuating internal electromagnetic fields, Altshuler et al. [8] obtained $1 / \tau_{n n}$ by first solving the differential equation for the Cooperon in a fixed electromagnetic field, and then averaging the result over different realizations of this field. The probability distribution for this averaging is constrained by the fluctuation-dissipation theorem. A similar strategy has recently been used by several authors to study Coulomb interactions in disordered metals [7, 23, 24, 25]. In all these works the solution of the equation for the Cooperon in a given electromagnetic field is represented as a Feynman path integral. After averaging over the electromagnetic field the evaluation of the path integral can be mapped onto the problem of solving a single-particle Schrödinger equation for a quantum particle in a certain effective potential. Altshuler et al. also found an exact expression for the WL-correction in the presence of an spatially homogeneous, time-dependent electric field [22]. From this result the dephasing contribution $1 / \tau_{A C}$ due to such a field was derived. 
In this chapter it will be shown that there exists an alternative method of solving the differential equation for the Cooperon in an external field. The method is based on an Eikonal expansion, which has been developed many years ago to calculate the single-particle Green's function of quantum field theories in fixed background fields [26, 27, 28]. One advantage of the Eikonal method is that it seems to be technically less complicated than the method used in Ref. [8], and the results can directly be compared with diagrammatic perturbation theory (see Sec.2.4). Furthermore it is free of any divergences for small momenta and frequencies, so that no additional cutoffs have to be introduced.

The rest of this chapter is organized as follows: In Sec.3.2 the Eikonal method is introduced. It provides an iteration procedure which formally solves the differential equations for the Cooperon and the Diffuson exactly. In practice, this procedure has to be truncated to obtain approximate solutions which still contain infinite orders in the external potential. This is outlined in Sec.3.3 and 3.4 where results for the Cooperon in a fluctuating random potential (Nyquist noise) and in an externally applied microwave field are derived. The dephasing time is extracted form these results via the weak localization correction to the conductivity. Finally in Sec. 3.5 the Eikonal method is applied to study the Diffuson in an external field. The influence of Nyquist noise on the Diffuson has also recently been studied by means of the path-integral technique [24, 33].

\subsection{The Cooperon in a fluctuating scalar poten- tial: Eikonal expansion}

As shown in Sec.2.3, Eq.(2.58), in a classical fluctuating scalar potential $V(\mathbf{r}, t)$ the Cooperon satisfies

$$
\left[\partial_{t}+D \hat{\mathbf{P}}_{\mathbf{r}}^{2}+\Gamma_{0}+i\left[V\left(\mathbf{r}, t_{0}+t\right)-V\left(\mathbf{r}, t_{0}-t\right)\right]\right] \mathcal{C}\left(\mathbf{r}, \mathbf{r}^{\prime} ; t, t^{\prime}, t_{0}\right)=\delta\left(\mathbf{r}-\mathbf{r}^{\prime}\right) \delta\left(t-t^{\prime}\right)
$$

where $\hat{\mathbf{P}}_{\mathbf{r}}=-i \nabla_{\mathbf{r}}$ is the momentum operator, and the phenomenological dephasing rate $\Gamma_{0}$ is due to processes that are not explicitly treated in this work, such as inelastic electron-phonon scattering. The potential $V(\mathbf{r}, t)$ is related to the longitudinal electric field via

$$
\mathbf{E}(\mathbf{r}, t)=-\nabla_{\mathbf{r}} \phi(\mathbf{r}, t), V(\mathbf{r}, t)=(-e) \phi(\mathbf{r}, t),
$$

where $-e$ is the charge of the electron.

Formally Eq.(3.1) looks like the differential equation for the imaginary-time single-particle Green's function of an electron in a fluctuating external potential

$$
V_{t_{0}}(\mathbf{r}, t):=i\left[V\left(\mathbf{r}, t_{0}+t\right)-V\left(\mathbf{r}, t_{0}-t\right)\right] .
$$


The real time version of this problem has been discussed extensively in the quantum field theory literature [26, 27]. Now a slight modification of the eikonal method developed by E. S. Fradkin [27] (see also Ref. [28]) is used to solve Eq.(3.1) without going through a path integral representation [8, 23, 24]. As a first step, a mixed representation is introduced, defining

$$
\mathcal{C}\left(\mathbf{r}, \mathbf{r}^{\prime} ; t, t^{\prime}, t_{0}\right)=\int \frac{d \mathbf{k}}{(2 \pi)^{d}} \int \frac{d \omega}{2 \pi} e^{i\left[\mathbf{k} \cdot\left(\mathbf{r}-\mathbf{r}^{\prime}\right)-\omega\left(t-t^{\prime}\right)\right]} \mathcal{C}(\mathbf{k}, \omega ; \mathbf{r}, t) .
$$

It is understood that $\mathcal{C}(\mathbf{k}, \omega ; \mathbf{r}, t)$ depends parametrically on $t_{0}$. From Eq.(3.1) one then obtains

$$
\left[\partial_{t}-i \omega+D\left(\hat{\mathbf{P}}_{\mathbf{r}}+\mathbf{k}\right)^{2}+\Gamma_{0}+V_{t_{0}}(\mathbf{r}, t)\right] \mathcal{C}(\mathbf{k}, \omega ; \mathbf{r}, t)=1
$$

Now the ansatz

$$
\mathcal{C}(\mathbf{k}, \omega ; \mathbf{r}, t)=\int_{0}^{\infty} d \tau e^{-\tau\left(\Gamma_{0}+D \mathbf{k}^{2}-i \omega\right)} Y(\mathbf{k}, \tau ; \mathbf{r}, t)
$$

is made. It is easy to see that this ansatz solves Eq.(3.5) if the auxiliary function $Y(\mathbf{k}, \tau ; \mathbf{r}, t)$ satisfies

$$
\left[\partial_{\tau}+\partial_{t}+D\left(\hat{\mathbf{P}}_{\mathbf{r}}^{2}+2 \mathbf{k} \cdot \hat{\mathbf{P}}_{\mathbf{r}}\right)+V_{t_{0}}(\mathbf{r}, t)\right] Y(\mathbf{k}, \tau ; \mathbf{r}, t)=0
$$

with boundary condition $Y(\mathbf{k}, 0 ; \mathbf{r}, t)=1$. Setting now

$$
Y(\mathbf{k}, \tau ; \mathbf{r}, t)=e^{-F(\mathbf{k}, \tau ; \mathbf{r}, t)},
$$

one obtains the eikonal equation

$$
\begin{array}{r}
{\left[\partial_{\tau}+\partial_{t}+D\left(\hat{\mathbf{P}}_{\mathbf{r}}^{2}+2 \mathbf{k} \cdot \hat{\mathbf{P}}_{\mathbf{r}}\right)\right] F(\mathbf{k}, \tau ; \mathbf{r}, t)=} \\
V_{t_{0}}(\mathbf{r}, t)+D\left[\hat{\mathbf{P}}_{\mathbf{r}} F(\mathbf{k}, \tau ; \mathbf{r}, t)\right]^{2}
\end{array}
$$

with boundary condition

$$
F(\mathbf{k}, 0 ; \mathbf{r}, t)=0 \text {. }
$$

Following Ref. [27] the solution of Eq.(3.9) is expanded in powers of the potential $V_{t_{0}}$,

$$
F(\mathbf{k}, \tau ; \mathbf{r}, t)=\sum_{n=1}^{\infty} F_{n}(\mathbf{k}, \tau ; \mathbf{r}, t),
$$

where $F_{n}$ involves by definition $n$ powers of $V_{t_{0}}$. It is easy to show that

$$
\left[\partial_{\tau}+\partial_{t}+D\left(\hat{\mathbf{P}}_{\mathbf{r}}^{2}+2 \mathbf{k} \cdot \hat{\mathbf{P}}_{\mathbf{r}}\right)\right] F_{n}(\mathbf{k}, \tau ; \mathbf{r}, t)=V_{n}(\mathbf{k}, \tau ; \mathbf{r}, t)
$$


where $V_{1}(\mathbf{k}, \tau ; \mathbf{r}, t) \equiv V_{t_{0}}(\mathbf{r}, t)$, and for $n \geq 2$

$$
V_{n}(\mathbf{k}, \tau ; \mathbf{r}, t)=D \sum_{n^{\prime}=1}^{n-1}\left[\hat{\mathbf{P}}_{\mathbf{r}} F_{n^{\prime}}(\mathbf{k}, \tau ; \mathbf{r}, t)\right] \cdot\left[\hat{\mathbf{P}}_{\mathbf{r}} F_{n-n^{\prime}}(\mathbf{k}, \tau ; \mathbf{r}, t)\right]
$$

In particular,

$$
V_{2}(\mathbf{k}, \tau ; \mathbf{r}, t)=D\left[\hat{\mathbf{P}}_{\mathbf{r}} F_{1}(\mathbf{k}, \tau ; \mathbf{r}, t)\right]^{2} .
$$

The solution of Eq. (3.12) with the correct boundary condition is

$$
F_{n}(\mathbf{k}, \tau ; \mathbf{r}, t)=\int_{0}^{\tau} d \tau^{\prime} e^{-\left(\tau-\tau^{\prime}\right)\left[\partial_{t}+D\left(\hat{\mathbf{P}}_{\mathbf{r}}^{2}+2 \mathbf{k} \cdot \hat{\mathbf{P}}_{\mathbf{r}}\right)\right]} V_{n}\left(\mathbf{k}, \tau^{\prime} ; \mathbf{r}, t\right) .
$$

The first term is explicitly

$$
F_{1}(\mathbf{k}, \tau ; \mathbf{r}, t)=\int \frac{d \mathbf{q}}{(2 \pi)^{d}} \frac{d \omega}{2 \pi} e^{i(\mathbf{q} \cdot \mathbf{r}-\omega t)} V_{t_{0}}(\mathbf{q}, \omega) f_{1}(\mathbf{k}, \tau ; \mathbf{q}, \omega),
$$

where

$$
f_{1}(\mathbf{k}, \tau ; \mathbf{q}, \omega)=\frac{1-e^{-\left[D\left(\mathbf{q}^{2}+2 \mathbf{k} \cdot \mathbf{q}\right)-i \omega\right] \tau}}{D\left(\mathbf{q}^{2}+2 \mathbf{k} \cdot \mathbf{q}\right)-i \omega}
$$

and $V_{t_{0}}(\mathbf{q}, \omega)$ is the Fourier transform of the potential $V_{t_{0}}(\mathbf{r}, t)$. Note that according to Eq. (3.3)

$$
V_{t_{0}}(\mathbf{q}, \omega)=i\left[e^{-i \omega t_{0}} V(\mathbf{q}, \omega)-e^{i \omega t_{0}} V(\mathbf{q},-\omega)\right]
$$

where

$$
V(\mathbf{q}, \omega)=\int d \mathbf{r} \int_{-\infty}^{\infty} d t e^{-i(\mathbf{q} \cdot \mathbf{r}-\omega t)} V(\mathbf{r}, t)
$$

For the quadratic term one obtains

$$
\begin{aligned}
F_{2}(\mathbf{k}, \tau ; \mathbf{r}, t)= & \int \frac{d \mathbf{q}_{1}}{(2 \pi)^{d}} \frac{d \omega_{1}}{2 \pi} \int \frac{d \mathbf{q}_{2}}{(2 \pi)^{d}} \frac{d \omega_{2}}{2 \pi} e^{i\left[\left(\mathbf{q}_{1}+\mathbf{q}_{2}\right) \cdot \mathbf{r}-\left(\omega_{1}+\omega_{2}\right)\right] t} V_{t_{0}}\left(\mathbf{q}_{1}, \omega_{1}\right) V_{t_{0}}\left(\mathbf{q}_{2}, \omega_{2}\right) \\
& \times f_{2}\left(\mathbf{k}, \tau ; \mathbf{q}_{1}, \omega_{1}, \mathbf{q}_{2}, \omega_{2}\right),
\end{aligned}
$$

with

$$
\begin{aligned}
& f_{2}\left(\mathbf{k}, \tau ; \mathbf{q}_{1}, \omega_{1}, \mathbf{q}_{2}, \omega_{2}\right)=\left(D \mathbf{q}_{1} \cdot \mathbf{q}_{2}\right) \frac{e^{-\left[D\left(\left(\mathbf{q}_{1}+\mathbf{q}_{2}\right)^{2}+2 \mathbf{k} \cdot\left(\mathbf{q}_{1}+\mathbf{q}_{2}\right)\right)-i\left(\omega_{1}+\omega_{2}\right)\right] \tau}}{\left[D\left(\mathbf{q}_{1}^{2}+2 \mathbf{k} \cdot \mathbf{q}_{1}\right)-i \omega_{1}\right]\left[D\left(\mathbf{q}_{2}^{2}+2 \mathbf{k} \cdot \mathbf{q}_{2}\right)-i \omega_{2}\right]} \\
& \times\left\{\frac{e^{\left[D\left(\left(\mathbf{q}_{1}+\mathbf{q}_{2}\right)^{2}+2 \mathbf{k} \cdot\left(\mathbf{q}_{1}+\mathbf{q}_{2}\right)\right)-i\left(\omega_{1}+\omega_{2}\right)\right] \tau}-1}{D\left(\left(\mathbf{q}_{1}+\mathbf{q}_{2}\right)^{2}+2 \mathbf{k} \cdot\left(\mathbf{q}_{1}+\mathbf{q}_{2}\right)\right)-i\left(\omega_{1}+\omega_{2}\right)}+\frac{e^{2 D \mathbf{q}_{1} \cdot \mathbf{q}_{2} \tau}-1}{2 D \mathbf{q}_{1} \cdot \mathbf{q}_{2}}\right. \\
& \left.-\frac{e^{\left[D\left(\mathbf{q}_{1}^{2}+2 \mathbf{k} \cdot \mathbf{q}_{1}+2 \mathbf{q}_{1} \cdot \mathbf{q}_{2}\right)-i \omega_{1}\right] \tau}-1}{D\left(\mathbf{q}_{1}^{2}+2 \mathbf{k} \cdot \mathbf{q}_{1}+2 \mathbf{q}_{1} \cdot \mathbf{q}_{2}\right)-i \omega_{1}}-\frac{e^{\left[D\left(\mathbf{q}_{2}^{2}+2 \mathbf{k} \cdot \mathbf{q}_{2}+2 \mathbf{q}_{2} \cdot \mathbf{q}_{1}\right)-i \omega_{2}\right] \tau}-1}{D\left(\mathbf{q}_{2}^{2}+2 \mathbf{k} \cdot \mathbf{q}_{2}+2 \mathbf{q}_{2} \cdot \mathbf{q}_{1}\right)-i \omega_{2}}\right\}
\end{aligned}
$$


Substituting the solution of Eq.(3.9) into Eqs.(3.8),(3.6), the $\omega$-integration in Eq.(3.4) can be performed exactly, and gives rise to a factor of $\delta\left(\tau-t+t^{\prime}\right)$. The $\tau$-integration in Eq.(3.6) becomes then trivial and yields the step function $\Theta\left(t-t^{\prime}\right)$. Eventually one obtains the following expression for the solution of Eq.(3.1),

$$
\mathcal{C}\left(\mathbf{r}, \mathbf{r}^{\prime} ; t, t^{\prime}, t_{0}\right)=\int \frac{d \mathbf{k}}{(2 \pi)^{d}} e^{i \mathbf{k} \cdot\left(\mathbf{r}-\mathbf{r}^{\prime}\right)} \mathcal{C}_{0}\left(\mathbf{k}, t-t^{\prime}\right) e^{-F\left(\mathbf{k}, t-t^{\prime} ; \mathbf{r}, t\right)}
$$

where $\mathcal{C}_{0}(\mathbf{k}, t)$ is the non-interacting Cooperon in the momentum-time domain, i.e.

$$
\mathcal{C}_{0}(\mathbf{k}, t)=\Theta(t) e^{-\left(\Gamma_{0}+D \mathbf{k}^{2}\right) t}
$$

Eqs.(3.22),(3.23) together with Eqs.(3.9),(3.10) complete the formal solution of Eq.(3.1).

\subsection{Dephasing due to Nyquist noise in arbitrary dimensions}

To identify the dephasing rate $1 / \tau_{\varphi}$ in terms of a physical observable, one usually calculates the weak localization correction to the conductivity [32]. The corresponding correction to the current is given in Eq.(2.68). To obtain the conductivity, Eq.(2.68) is expressed in terms of the Fourier components of $\mathbf{E}_{d r i}(t)=\int \frac{d \varepsilon^{\prime}}{2 \pi} \mathbf{E}_{d r i}\left(\varepsilon^{\prime}\right) e^{-i \varepsilon^{\prime} t}$. This yields

$$
\delta \mathbf{j}_{W L}(\varepsilon)=\int \frac{d \varepsilon^{\prime}}{2 \pi} \delta \sigma_{W L}\left(\varepsilon, \varepsilon^{\prime}\right) \mathbf{E}_{d r i}\left(\varepsilon^{\prime}\right)
$$

with

$$
\delta \sigma_{W L}\left(\varepsilon, \varepsilon^{\prime}\right)=-\frac{\sigma_{d}}{\pi \nu_{d}} \int_{-\infty}^{\infty} d t e^{i \frac{\varepsilon+\varepsilon^{\prime}}{2} t} \int_{-\infty}^{\infty} d t_{0} e^{i\left(\varepsilon-\varepsilon^{\prime}\right) t_{0}} \mathcal{C}\left(\mathbf{r}, \mathbf{r} ; \frac{t}{2},-\frac{t}{2}, t_{0}\right) .
$$

$\delta \sigma_{W L}\left(\varepsilon, \varepsilon^{\prime}\right)$ is the generalized weak-localization correction to the conductivity in the presence of external dephasing fields. The dephasing time is defined as the time scale that cuts off the $t$-integration in Eq.(3.25). This will be discussed in detail when performing the explicit calculations below. So far the nature of the potential $V(\mathbf{r}, t)$ in Eq.(3.1) was not specified. If one assumes that it describes equilibrium fluctuations due to electron-electron interactions (Nyquist noise) [8], one may use Eq.(3.1) to calculate the dephasing rate in disordered metals due to electron-electron interactions with small momentum transfers. Such a calculation has first been performed in the pioneering paper by Altshuler, Aronov and Khmelnitskii [8]. The dephasing time in disordered systems has lately attracted great interest again, since new measurements indicate, that $1 / \tau_{\varphi}$ saturates at low temperatures [19]. In these 
experiments great care has been taken to rule out unwanted effects like magnetic impurities or heating and it has been proposed, that the observed saturation is due to intrinsic interaction effects [19]. This contradicts the conventional theory [8] which predicts, that $1 / \tau_{\varphi}$ vanishes as some power of temperature, depending on the dimensionality of the system under investigation. An attempt to explain the experimentally observed saturation due to electron-electron interactions [38] has been heavyly criticized [32, 43, 25]. It has been argued, that the measured effect is not of intrinsic nature but rather due to external microwave-radiation, which is unintentionally coupled to the system. New experiments, in which the effect of external electro-magnetic radiation is directly investigated [44] do not support this claim. Another attempt to explain the saturation in the framework of the "conventional" theory takes into account the possible dynamics of the impurities which are usually assumed to be static scattering centers [37]. Still there is no general agreement about the mechanism that is responsible for the experimentally obtained behavior of the dephasing time [39]. While in Ref. [38] the electron-electron interaction is treated quantum mechanically from the beginning, Ref. [8] deals only with the classical fluctuations of the background field. Quantum mechanics is brought in by introducing a high frequency cutoff to take into account the Pauli principle. The main statement of Ref. [38] is, that this cutoff procedure is not correct and the Pauli principle does not affect the dephasing time.

In this section both, the results of Ref. [8] and Ref. [38] which, within the Eikonal formalism only differ in the high frequency cutoff, will be rederived. Since in this work the external field is treated classically, this cutoff has to be introduced by hand into the calculations presented here and a proper justification of the chosen value can not be given within this framework. However, the aim of this section is not to enter the debate about zero temperature dephasing, but rather to point out important differences between the two results. Especially the role of vertex corrections becomes very clear in the Eikonal expansion. It turns out, that vertex corrections are essential to obtain the conventional result [8, 32, 43] but play no role for the zero temperature term [38]. Some consequences from this fact are outlined which indicate, that the restriction to first order perturbation theory in Ref. [43] is probably not responsible for the discrepancy to the non-perturbative calculation in Ref. [38]. Moreover, the dephasing rate for arbitrary dimension $d$, treating $d$ as a continuous parameter, will be calculated. In this way one sees that the logarithmic corrections in $d=2$ are accompanied with large numerical prefactors for $0<|d-2| \ll 1$, which diverge as $|d-2|^{-1}$ for $d \rightarrow 2$.

To obtain the Cooperon of the interacting system, one first solves Eq. (3.1) for a fixed realization of the field, and then averages the solution over all realizations of the field, with the correlator given by the fluctuation-dissipation theorem [8], which implies (see App.B)

$$
\left\langle V(\mathbf{q}, \omega) V\left(\mathbf{q}^{\prime}, \omega^{\prime}\right)\right\rangle_{n n}=(2 \pi)^{d+1} \delta\left(\mathbf{q}+\mathbf{q}^{\prime}\right) \delta\left(\omega+\omega^{\prime}\right) g(\mathbf{q}, \omega)
$$


where

$$
g(\mathbf{q}, \omega)=-\operatorname{coth}\left(\frac{\omega}{2 T}\right) \operatorname{Im}\left[\frac{f_{\mathbf{q}}}{\epsilon(\mathbf{q}, \omega)}\right] .
$$

$\langle\cdots\rangle_{n n}$ denotes averaging over the Nyquist noise probability distribution. Here $f_{\mathbf{q}}$ is the Fourier transform of the bare Coulomb interaction, and $\epsilon(\mathbf{q}, \omega)$ is the disorder averaged dielectric function of the system. In the diffusive regime it is given by Eq.(2.82). Recall, that in the derivation of Eq.(3.1) the electromagnetic field is treated as a classical field [25]. The fluctuation-dissipation theorem can thus not be applied for arbitrarily large frequencies $\omega$. The question arises, at which maximal frequency $\omega_{c}$ the $\omega$-integrations have to be cut off to be consistent with the classical approximation. According to Altshuler et al. [8] $\omega_{c}$ should be chosen as $\omega_{c}=\max \{T, \varepsilon\}$, where $T$ is the temperature and $\varepsilon$ is the frequency of an external measuring field $\mathbf{E}_{d r i}(\varepsilon)$, to explicitly take into account the Pauli principle. The intuitive argument behind this is the following: According to Fermi's golden rule of quantum mechanics, an external field of frequency $\omega$ can only cause transitions between states with energy difference $\pm \omega$. Now one is interested in transitions caused by the fluctuating field of the background electrons. If one assumes, that this field can cause transitions with energy $\omega>T$, then there must be some background particles with such energy. But since the whole system is in equilibrium at temperature $T$, the probability to find such particles is exponentially small. In non-linear order the time-dependent driving field $\mathbf{E}_{d r i}(\varepsilon)$ with $\varepsilon>0$ will in principle also contribute to dephasing [56,57]. The total dephasing time consists in this case of an 'external' contribution from $\mathbf{E}_{d r i}$, and an 'intrinsic' contribution from electron-electron interactions. Which effect dominates depends on the amplitude and frequency of the external field [57]. (This point will be discussed in Chapter 4, where the non-linear response of a mesoscopic metal ring to a time-dependent external flux is studied.) First only the intrinsic contribution due to Nyquist Noise is investigated. For $\varepsilon>T$ the external frequency replaces the temperature as the relevant energy scale. As already mentioned, the results of Golubev and Zaikin [38] contradict the above choice for $\omega_{c}$. The method used in Ref. [38] takes into account the full quantum mechanical character of the interaction, but is formally related to the derivation presented here in that sense, that the effect of the electron-electron interaction is mapped onto a fluctuating external field due to a Hubbard-Stratonovich transformation. It will be demonstrated further below, that the result of Ref. [38, 39] can be obtained from the Eikonal approximation, if one chooses $\omega_{c}$ as the inverse of the elastic lifetime $\tau_{e l}$, i.e. $\omega_{c}=1 / \tau_{e l}$, which is the frequency that limits the validity of the diffusive approximation. Within this approximation $g(\mathbf{q}, \omega)$ is given by (see Eq.(2.82))

$$
g(\mathbf{q}, \omega) \approx \operatorname{coth}\left(\frac{\omega}{2 T}\right) \frac{\omega}{\nu_{d} D \mathbf{q}^{2}} \text { for } \omega<\frac{1}{\tau_{e l}} \text { and }|\mathbf{q}|<\frac{1}{l} .
$$

Because the momentum integrals in the following analysis will be infrared and ultraviolet convergent, the frequency dependence in the denominator of $g(\mathbf{p}, \omega)$ was 
neglected and no further cutoffs for the $\mathbf{q}$-integrations are needed in this calculation. Note that Altshuler et al. [8] have to introduce some ad hoc (but physically motivated) ultraviolet cutoff for momentum integrations in order to calculate dephasing due to Nyquist noise in $d \geq 2$. The Eikonal approach is free of such ambiguities. Applying Eq.(3.26) to the potential $V_{t_{0}}(\mathbf{q}, \omega)$ which enters the differential equation for the Cooperon, one obtains from Eq.(3.3)

$$
\begin{aligned}
\left\langle V_{t_{0}}(\mathbf{q}, \omega) V_{t_{0}}\left(\mathbf{q}^{\prime}, \omega^{\prime}\right)\right\rangle_{n n} & =-2(2 \pi)^{d+1} \delta\left(\mathbf{q}+\mathbf{q}^{\prime}\right) \\
& \times\left[\delta\left(\omega+\omega^{\prime}\right)-\delta\left(\omega-\omega^{\prime}\right)\right] g(\mathbf{q}, \omega) .
\end{aligned}
$$

Thus the correlator of $V_{t_{0}}(\mathbf{q}, \omega)$ is independent of the time $t_{0}$. This implies, that after averaging the Cooperon is independent of $t_{0}$ as well. It is important to note, that Eq. (3.29) consists of a difference of two terms. In Sec.3.3.4 the result from first order perturbation theory (see Chap.2.4) will be rederived from the Eikonal expansion. There it becomes clear, that the first term in Eq. $(3.29) \sim \delta\left(\omega+\omega^{\prime}\right)$ describes selfenergy corrections (see Fig.2.9(a,b)), while the second one $\sim \delta\left(\omega-\omega^{\prime}\right)$ is responsible for vertex corrections (see Fig.2.9(c,d)). The Cooperon of the interacting many-body system is denoted by $C\left(\mathbf{r}, \mathbf{r}^{\prime}, t, t^{\prime}\right)$, i.e.

$$
C\left(\mathbf{r}, \mathbf{r}^{\prime}, t, t^{\prime}\right)=\left\langle\mathcal{C}\left(\mathbf{r}, \mathbf{r}^{\prime} ; t, t^{\prime}, t_{0}\right)\right\rangle_{n n} .
$$

Now, after averaging over the external field Eq.(3.24) obviously reduces to

$$
\delta \mathbf{j}_{W L}(\varepsilon)=\delta \sigma_{W L}(\varepsilon) \mathbf{E}_{d r i}(\varepsilon),
$$

with

$$
\delta \sigma_{W L}(\varepsilon)=-\frac{\sigma_{d}}{\pi \nu_{d}} \int_{-\infty}^{\infty} d t C\left(\mathbf{r}, \mathbf{r}, \frac{t}{2},-\frac{t}{2}\right) e^{i \varepsilon t}
$$

If Eq.(3.22) is averaged over a suitable probability distribution of the field and one assumes that averaging restores spatial translational invariance, then the average of the Eikonal factor in Eq.(3.22) must be independent of $\mathbf{r}$, so that one obtains for the Cooperon in the momentum-time domain

$$
C\left(\mathbf{k}, t, t^{\prime}\right)=C_{0}\left(\mathbf{k}, t-t^{\prime}\right) e^{-W\left(\mathbf{k}, t-t^{\prime} ; t\right)},
$$

with $C\left(\mathbf{r}, \mathbf{r}^{\prime}, t, t^{\prime}\right)=\int \frac{d \mathbf{k}}{(2 \pi)^{d}} e^{i \mathbf{k}\left(\mathbf{r}-\mathbf{r}^{\prime}\right)} C\left(\mathbf{k}, t, t^{\prime}\right)$ and

$$
W\left(\mathbf{k}, t-t^{\prime} ; t\right)=-\ln \left\langle e^{-F\left(\mathbf{k}, t-t^{\prime} ; \mathbf{r}, t\right)}\right\rangle_{n n} .
$$

Note that according to Eq.(3.32) the weak-localization correction to the conductivity can be written as

$$
\frac{\delta \sigma_{W L}(\varepsilon)}{\sigma_{d}}=-\frac{1}{\pi \nu_{d}} \int_{\tau_{e l}}^{\infty} d t \int \frac{d \mathbf{k}}{(2 \pi)^{d}} e^{-\left(\Gamma_{0}+D \mathbf{k}^{2}-i \varepsilon\right) t-\Gamma(\mathbf{k}, t)},
$$


where

$$
\Gamma(\mathbf{k}, t)=W(\mathbf{k}, t ; t / 2)=-\ln \left\langle e^{-F(\mathbf{k}, t ; \mathbf{r}, t / 2)}\right\rangle_{n n} .
$$

The lower cutoff $1 / \tau_{e l}$ in the time integration is introduced, since the Cooperon is only defined in the diffusive regime with $t>\tau_{e l}$. Without interactions $\Gamma(\mathbf{k}, t)$ vanishes and the time integral in Eq.(3.35) is cut off by $\Gamma_{0}$. Now if $\Gamma(\mathbf{k}, t)$ would have a $\mathbf{k}$ independent contribution linear in $t$; i.e. if one could write $\Gamma(\mathbf{k}=\mathbf{0}, t)=\Gamma_{1} t$; the correction to $\Gamma_{0}$ due to Nyquist noise would obviously be given by $\Gamma_{1}$. Generally the time-scale that cuts off the $t$-integration is determined by the $\mathbf{k}$ independent part $\Gamma(\mathbf{k}=\mathbf{0}, t)$ and in the following the $\mathbf{k}$-dependence will therefore not be considered. However, a priori it is not clear whether $\Gamma(\mathbf{k}=\mathbf{0}, t)$ grows linearly in $t$ or not. In fact, it will be shown shortly that for long-range Coulomb interactions in $d \leq 2$ this is not the case.

\subsubsection{The Debye-Waller factor}

So far no approximation has been made. Of course, the function $F(\mathbf{k}, \tau ; \mathbf{r}, t)$ cannot be calculated exactly, and one has to use the iterative procedure outlined above and expand $F$ in powers of the potential $V_{t_{0}}$. To first order in the screened Coulomb interaction, it is sufficient to truncate the expansion of $F$ at the second order, $F \approx$ $F_{1}+F_{2}$. To include all second order contributions in the exponential factor, one performs the cumulant expansion

$$
\left\langle e^{-F}\right\rangle_{n n} \approx e^{-\langle F\rangle_{n n}+\frac{1}{2}\left\langle\left(F-\langle F\rangle_{n n}\right)^{2}\right\rangle_{n n}} .
$$

In this approximation the function $\Gamma(\mathbf{k}, t)$ defined in Eq.(3.36) is given by

$$
\begin{gathered}
\Gamma(\mathbf{k}, t)=\Gamma_{1}(\mathbf{k}, t)+\Gamma_{2}(\mathbf{k}, t), \\
\Gamma_{1}(\mathbf{k}, t)=-\frac{1}{2}\left\langle F_{1}^{2}(\mathbf{k}, t ; \mathbf{r}, t / 2)\right\rangle_{n n}, \\
\Gamma_{2}(\mathbf{k}, t)=\left\langle F_{2}(\mathbf{k}, t ; \mathbf{r}, t / 2)\right\rangle_{n n} .
\end{gathered}
$$

Here it was made use of $\left\langle V_{t_{0}}(\mathbf{q}, \omega)\right\rangle_{n n}=0$. The averaging is now easily performed with the help of the fluctuation-dissipation theorem (3.26), which leads to the correlator of the potential $V_{t_{0}}(\mathbf{q}, \omega)$ as given in Eq.(3.29). Introducing for simplicity the notation

$$
E_{\mathbf{k}}(\mathbf{q})=D\left(\mathbf{q}^{2}+2 \mathbf{k} \cdot \mathbf{q}\right)
$$


one obtains

$$
\begin{aligned}
\Gamma_{1}(\mathbf{k}, t)= & -\frac{1}{2}\left\langle F_{1}^{2}(\mathbf{k}, t ; \mathbf{r}, t / 2)\right\rangle_{n n} \\
= & \int \frac{d \mathbf{q}}{(2 \pi)^{d}} \int_{-\infty}^{\infty} \frac{d \omega}{2 \pi} g(\mathbf{q}, \omega)\left\{\frac{1-e^{-\left(E_{\mathbf{k}}(\mathbf{q})-i \omega\right) t}}{E_{\mathbf{k}}(\mathbf{q})-i \omega} \frac{1-e^{-\left(E_{\mathbf{k}}(-\mathbf{q})+i \omega\right) t}}{E_{\mathbf{k}}(-\mathbf{q})+i \omega}\right. \\
& \left.\quad-e^{-i \omega t} \frac{1-e^{-\left(E_{\mathbf{k}}(\mathbf{q})-i \omega\right) t}}{E_{\mathbf{k}}(\mathbf{q})-i \omega} \frac{1-e^{-\left(E_{\mathbf{k}}(-\mathbf{q})-i \omega\right) t}}{E_{\mathbf{k}}(-\mathbf{q})-i \omega}\right\}, \\
\Gamma_{2}(\mathbf{k}, t)= & \left\langle F_{2}(\mathbf{k}, t ; \mathbf{r}, t / 2)\right\rangle_{n n} \\
= & 2 \int \frac{d \mathbf{q}}{(2 \pi)^{d}} \int_{-\infty}^{\infty} \frac{d \omega}{2 \pi} g(\mathbf{q}, \omega) D \mathbf{q}^{2}\left\{\frac{1}{\left[E_{\mathbf{k}}(\mathbf{q})-i \omega\right]\left[E_{\mathbf{k}}(-\mathbf{q})+i \omega\right]}\right] t \\
+ & \left.\frac{1-e^{-2 D \mathbf{q}^{2} t}}{2 D \mathbf{q}^{2}}-\frac{1-e^{-\left(E_{\mathbf{k}}(\mathbf{q})-i \omega\right) t}}{E_{\mathbf{k}}(\mathbf{q})-i \omega}-\frac{1-e^{-\left(E_{\mathbf{k}}(-\mathbf{q})+i \omega\right) t}}{E_{\mathbf{k}}(-\mathbf{q})+i \omega}\right] \\
& -\frac{1}{\left[E_{\mathbf{k}}(\mathbf{q})-i \omega\right]\left[E_{\mathbf{k}}(-\mathbf{q})-i \omega\right]}\left[\frac{\sin (\omega t)}{\omega}+e^{i \omega t}\left[\frac{1-e^{-2 D \mathbf{q}^{2} t}}{2 D \mathbf{q}^{2}}\right.\right. \\
& \left.\left.\left.-\frac{1-e^{-\left(E_{\mathbf{k}}(\mathbf{q})+i \omega\right) t}}{E_{\mathbf{k}}(\mathbf{q})+i \omega}-\frac{1-e^{-\left(E_{\mathbf{k}}(-\mathbf{q})+i \omega\right) t}}{E_{\mathbf{k}}(-\mathbf{q})+i \omega}\right]\right]\right\}
\end{aligned}
$$

Adding $\Gamma_{1}$ and $\Gamma_{2}$ the single terms can be grouped together. Exploiting the freedom to change the integration variables according to $\mathbf{q} \rightarrow-\mathbf{q}$ and $\omega \rightarrow-\omega$ and performing some algebraic manipulations, $\Gamma(\mathbf{k}, t)$ can be written in the compact form

$$
\begin{aligned}
\Gamma(\mathbf{k}, t)=2 \operatorname{Re} \int \frac{d \mathbf{q}}{(2 \pi)^{d}} \int_{-\infty}^{\infty} \frac{d \omega}{2 \pi} g(\mathbf{q}, \omega)\left[\frac{t}{E_{\mathbf{k}}(\mathbf{q})-i \omega}\right. \\
\left.+\frac{e^{-\left(E_{\mathbf{k}}(\mathbf{q})-i \omega\right) t}-1}{\left(E_{\mathbf{k}}(\mathbf{q})-i \omega\right)^{2}}-\frac{e^{-E_{\mathbf{k}}(\mathbf{q}) t}-e^{-i \omega t}}{i \omega\left(E_{\mathbf{k}}(\mathbf{q})-i \omega\right)}\right] .
\end{aligned}
$$

It is important to note, that the first two terms in this equation arise from the contribution $\sim \delta\left(\omega+\omega^{\prime}\right)$ in Eq.(3.29), while the last term comes from the part $\sim$ $\delta\left(\omega-\omega^{\prime}\right)$. It was already mentioned, that these are just the contributions due to selfenergy and vertex corrections respectively, which will become clear from comparison with first order perturbation theory (and will be shown in Sec. 3.3.4). As discussed above, for obtaining the interaction corrections to the dephasing rate it is sufficient to set $\mathbf{k}=0$ in Eqs.(3.44). Introducing the notation $\Gamma(t):=\Gamma(\mathbf{k}=\mathbf{0}, t)$ one gets

$$
\begin{array}{r}
\Gamma(t)=\frac{4}{\nu_{d}} \int \frac{d \mathbf{q}}{(2 \pi)^{d}} \int_{0}^{\omega_{c}} \frac{d \omega}{2 \pi} \operatorname{coth}\left(\frac{\omega}{2 T}\right) \frac{\omega}{D \mathbf{q}^{2}}\left[\frac{D \mathbf{q}^{2}}{\left(D \mathbf{q}^{2}\right)^{2}+\omega^{2}}\left(t-\frac{\sin (\omega t)}{\omega}\right)+\right. \\
\left.+\operatorname{Re} \frac{e^{-\left(D \mathbf{q}^{2}-i \omega\right) t}-1}{\left(D \mathbf{q}^{2}-i \omega\right)^{2}}-\frac{e^{-D \mathbf{q}^{2} t}-\cos (\omega t)}{\left(D \mathbf{q}^{2}\right)^{2}+\omega^{2}}\right]
\end{array}
$$


where $g(\mathbf{q}, \omega)$ was inserted according to Eq.(3.28). Eq.(3.45) was derived for macroscopic systems since all momentum sums where replaced by integrals; $\frac{1}{L^{d}} \sum_{\mathbf{k}} \rightarrow$ $\int \frac{d \mathbf{k}}{(2 \pi)^{d}}$. Here it is summed over diffusive modes only. However, the derivation also holds for mesoscopic systems if only the integrals are replaced by discrete sum. This yields the more general expression

$$
\begin{array}{r}
\Gamma(t)=\frac{4}{\nu_{d}} \frac{1}{L^{d}} \sum_{\mathbf{q} \neq \mathbf{0}} \int_{0}^{\omega_{c}} \frac{d \omega}{2 \pi} \operatorname{coth}\left(\frac{\omega}{2 T}\right) \frac{\omega}{D \mathbf{q}^{2}}\left[\frac{D \mathbf{q}^{2}}{\left(D \mathbf{q}^{2}\right)^{2}+\omega^{2}}\left(t-\frac{\sin (\omega t)}{\omega}\right)+\right. \\
\left.+\operatorname{Re} \frac{e^{-\left(D \mathbf{q}^{2}-i \omega\right) t}-1}{\left(D \mathbf{q}^{2}-i \omega\right)^{2}}-\frac{e^{-D \mathbf{q}^{2} t}-\cos (\omega t)}{\left(D \mathbf{q}^{2}\right)^{2}+\omega^{2}}\right] .
\end{array}
$$

The $\mathbf{q}=\mathbf{0}$ mode is excluded, since the system is assumed to be over all charge neutral [40], [30]. Capacitance effects, involving the zeroth Fourier component of the Coulomb potential, are not considered here. Eq.(3.46), Eq.(3.45) respectively, presents a compact expression, which is free of any divergences and allows to calculate the dephasing time $\tau_{\varphi}$ for various physical situations from the condition $\Gamma_{0}+\Gamma\left(\tau_{\varphi}\right)=1$. In the following $\tau_{\varphi}$ will be calculated in infinite and finite systems for $T \gg \varepsilon$ as well as for $\varepsilon \gg T$. Choosing $\omega_{c}=\max \{T, \varepsilon\}$ and $\omega_{c} \tau_{\varphi} \gg 1$, all results of the conventional theory [8], [40], [30] are reproduced. For $\varepsilon \gg T$ the dephasing time in arbitrary dimensions is obtained. Assuming $\omega_{c}=1 / \tau_{\text {el }}$ reproduces the result recently obtained in Ref. [38] for $d=1$. First the case of an infinite system is considered.

\subsubsection{The dephasing rate in infinite systems}

$$
\text { a) } T \gg \varepsilon
$$

For $T \gg \varepsilon$ the approximation $\varepsilon \approx 0$ is made in Eq.(3.45). To reproduce the conventional results [8], one has to choose $\omega_{c}=T$, thus taking into account only the classical Nyquist noise fluctuations. The corresponding contribution to the dephasing time is denoted by $\tau_{n n}$. Since the frequency integration is now restricted to $\omega<T$ one can approximate $\operatorname{coth}(\omega /(2 T)) \approx 2 T / \omega$ and investigate the asymptotic behavior for $T \tau_{n n} \gg 1$. Going over to $d$-dimensional spherical coordinates and substituting $x:=D p^{2} t$ and $y:=\omega t$ in Eq.(3.45) one arrives at

$$
\begin{aligned}
\Gamma(t)=\frac{4 T K_{d} t^{2-\frac{d}{2}}}{2 \pi \nu_{d} D^{\frac{d}{2}}} \int_{0}^{\infty} & d x \int_{0}^{T t} d y x^{\frac{d}{2}-1}\left[\frac{1}{x^{2}+y^{2}}\left(1-\frac{\sin y}{y}\right)\right. \\
+ & \left.\operatorname{Re} \frac{1}{x}\left(\frac{e^{-(x-i y)}-1}{(x-i y)^{2}}-\frac{e^{-x}-e^{i y}}{x^{2}+y^{2}}\right)\right] .
\end{aligned}
$$

$K_{d}=2 \pi^{d / 2} /\left[(2 \pi)^{d} \Gamma(d / 2)\right]$ (here $\Gamma(x)$ is the Gamma function) is the surface of the $d$-dimensional unit sphere divided by $(2 \pi)^{d}$, i.e. $K_{1}=1 / \pi, K_{2}=1 /(2 \pi)$ and 
$K_{3}=1 /\left(2 \pi^{2}\right)$. In $d<2$ both integrals exist for $T t \rightarrow \infty$ and can be calculated exactly in this limit. The result is

$$
\Gamma(t)=C_{d} \frac{T t^{2-\frac{d}{2}}}{\nu_{d} D^{\frac{d}{2}}} \quad \text { for } d<2 \text { and } T t \gg 1,
$$

where the constant $C_{d}$ is given by

$$
C_{d}=\frac{2^{3-d}}{\pi^{\frac{d}{2}}(2-d)(4-d)} .
$$

Obviously $C_{d}$ diverges for $d \rightarrow 2$. Because in $d<2$ the function $\Gamma(t)$ grows for large $t$ faster than linear, the term $\Gamma_{0} t$ in Eq.(3.35) is negligible in this case. The total dephasing rate $1 / \tau_{\varphi}$ is dominated by $1 / \tau_{n n}$ which may then be defined by

$$
\Gamma\left(\tau_{n n}\right)=1
$$

This yields

$$
\frac{1}{\tau_{n n}}=\left(\frac{C_{d} T}{\nu_{d} D^{\frac{d}{2}}}\right)^{\frac{2}{4-d}} \quad \text { for } d<2
$$

In two dimensions the first term in the braces in Eq.(3.47) diverges logarithmically for $T t \rightarrow \infty$, while the remaining term exists in this limit. The asymptotic behavior for $T t \gg 1$ is thus given by

$$
\begin{aligned}
\Gamma(t) & =\frac{4 T K_{2} t}{2 \pi \nu_{2} D} \int_{0}^{\infty} d x \int_{0}^{T t} d y \frac{1}{x^{2}+y^{2}}\left(1-\frac{\sin y}{y}\right) \\
& =\frac{4 T K_{2} t}{2 \pi \nu_{2} D} \int_{0}^{T t} d y \frac{\pi}{2 y}\left(1-\frac{\sin y}{y}\right) \approx \frac{T t}{2 \pi \nu_{2} D} \ln (T t)
\end{aligned}
$$

Due to the logarithmic correction the term $\Gamma_{0} t$ in Eq. (3.35) is still negligible, so that the total dephasing rate in $d=2$ is still given by $1 / \tau_{\varphi} \approx 1 / \tau_{n n}$ and can be obtained from Eq.(3.50), which leads to the self-consistency condition

$$
\frac{1}{\tau_{n n}}=\frac{T}{2 \pi \nu_{2} D} \ln \left(T \tau_{n n}\right)
$$

Iterating this equation one obtains

$$
\frac{1}{\tau_{n n}}=\frac{T}{2 \pi \nu_{2} D}\left[\ln \left(2 \pi \nu_{2} D\right)+\mathcal{O}\left(\ln \ln \left(2 \pi \nu_{2} D\right)\right)\right]
$$

in agreement with Ref. [8]. Note that in a good metal the parameter $2 \pi \nu_{2} D=k_{F} l=$ $2 \varepsilon_{F} \tau_{e l}\left(\varepsilon_{F}\right.$ is the Fermi energy) is large compared with unity (taking the spin into account, one has $\nu_{2}=m / \pi$ in a truely two dimensional sample). 
It is easy to see that in $d>2$ the long-time behavior of the Debye-Waller factor $\Gamma(t)$ is dominated by the term linear in $t$ on the right hand side of Eq.(3.45), i.e.

$$
\Gamma(t) \approx 4 t \int \frac{d \mathbf{q}}{(2 \pi)^{d}} \int_{0}^{T} \frac{d \omega}{2 \pi} \frac{g(\mathbf{q}, \omega) D \mathbf{q}^{2}}{\left[D \mathbf{q}^{2}-i \omega\right]\left[D \mathbf{q}^{2}+i \omega\right]} .
$$

Because in $d>2$ this integral exists, the contribution from Coulomb interactions with small momentum transfers to the dephasing rate is simply obtained by writing $\Gamma(t)=t / \tau_{n n}$. Hence,

$$
\frac{1}{\tau_{n n}}=\int \frac{d \mathbf{q}}{(2 \pi)^{d}} \int_{-T}^{T} d \omega g(\mathbf{q}, \omega) S(\mathbf{q}, \omega)
$$

where $S(\mathbf{q}, \omega)$ is the dynamic structure factor of the diffusing electrons in the regime $|\omega| \lesssim T$

$$
S(\mathbf{q}, \omega)=\frac{1}{\pi} \frac{D \mathbf{q}^{2}}{\left(D \mathbf{q}^{2}\right)^{2}+\omega^{2}}
$$

Note that for $|\omega| \lesssim T$ the detailed balance factor $e^{-\omega / T}$ in the dynamic structure factor can be replaced by unity. Eq.(3.56) agrees with the expression for the dephasing rate given in a recent paper by Imry et al. [37] (see also Ref. [5]). It should be kept in mind, however, that Eq.(3.56) is only valid as long as the integral does not exhibit any infrared divergence. For equilibrium Nyquist noise with correlator $g(\mathbf{q}, \omega)$ given in Eq. (3.27) the integral diverges for $d \leq 2$. In $d=2$ the integral depends in addition logarithmically on the upper cutoff $t T$, which leads to the logarithmic correction in Eq.(3.52). On the other hand, the full expression for $\Gamma(t)$ given in Eq.(3.45) is free of infrared divergences, so that this equation is more general than the semiclassical expression (3.56).

The dephasing rate in $d>2$ is now easily calculated. Evaluating the integral in Eq.(3.56) yields

$$
\frac{1}{\tau_{n n}}=\tilde{C}_{d} \frac{T^{\frac{d}{2}}}{\nu_{d} D^{\frac{d}{2}}}
$$

where

$$
\tilde{C}_{d}=\frac{K_{d}}{\left(\frac{d}{2}-1\right) \sin (\pi d / 4)}
$$

and the total dephasing rate in $d>2$ is given by $1 / \tau_{\varphi}=\Gamma_{0}+1 / \tau_{n n}$. $\tilde{C}_{d}$ diverges for $d \rightarrow 2$, just as $C_{d}$, indicating logarithmic corrections in $d=2$. Assuming that $\Gamma_{0}$ is dominated by electron-electron interactions with large momentum transfers, simple dimensional analysis [8, 34] implies $\Gamma_{0} \propto T^{d / 2}$, so that in $d=3$ both terms in Eq.(3.58) have the same order of magnitude. However, the contribution from 
electron-electron interactions with small momentum transfers becomes more and more important as one approaches $d \rightarrow 2$, and eventually dominates for $d \leq 2$.

So far it was shown, that the conventional results for the dephasing time are correctly reproduced by the Eikonal approximation. To obtain these results it was crucial to choose the frequency cutoff as $\omega_{c}=T$. In Ref. [32] $\tau_{n n}$ was derived in first order perturbation theory in the screened interaction and under the condition $\tau_{n n} \Gamma_{0} \gg 1$ with the results given in Eq.(2.97). It is emphasized that these perturbative expansions are only valid if $\Gamma_{0} \gg 1 / \tau_{n n}$. In contrast to that, the Eikonal result is free of any singularities even in the limit $\Gamma_{0} \rightarrow 0$, where the perturbative results for $d=1$ and $d=2$ of Eq.(2.97) are divergent. Nevertheless it is easy to see that these equations reproduce the results derived above, if $\Gamma_{0}$ is self-consistently replaced by $1 / \tau_{n n}$. Thus the validity of the procedure used in Ref. [32], which is a priori perturbative in the parameter $1 /\left(\Gamma_{0} \tau_{n n}\right)$, holds even beyond this perturbative regime. For $d=1$ this has already been proven in Ref. [25].

Recently Golubev and Zaikin [39] have used a path integral formalism to calculate a function $f(t)$, which is defined analogous to the function $\Gamma(t)$. In $d=1$ the authors found a linear in time but temperature independent contribution to $f(t)$, which is equivalent to a constant dephasing time at $T=0$. Moreover several sub-dominant contributions to the conventional result given in Eq.(3.48) were derived. The result of Ref. [39] can be reproduced within the Eikonal-approximation if one chooses $\omega_{c}=$ $1 / \tau_{\text {el }}$. To obtain the leading behavior, the approximations $\operatorname{coth}[\omega /(2 T)] \approx 2 T / \omega$ for $|\omega| \leq 2 T$ and $\operatorname{coth}[\omega /(2 T)] \approx 1$ for $|\omega| \geq 2 T$ are made. In order to compare the results with the ones of Ref. [38] the dimension $d=1$ is investigated. Since in this case the integral in Eq.(3.47) does not depend on the cutoff $T$, the region $|\omega| \leq 2 T$ simply yields the result given in Eq.(3.51): $\Gamma(t) \sim T t^{3 / 2} /\left(\nu_{1} \sqrt{D}\right)$. The remaining contribution from the region $|\omega| \geq 2 T$ is denoted by $\Delta \Gamma(t)$.

In the limit $T t \gg 1$ the oscillating terms can be neglected. Integrating the second term in Eq.(3.45) by parts one arrives at

$$
\begin{gathered}
\Delta \Gamma(t) \approx \frac{4}{\nu_{1}} \int_{-\infty}^{\infty} \frac{d p}{2 \pi}\left\{\frac{1}{D p^{2}}\left[\frac{(2 T)^{2}}{\left(D p^{2}\right)^{2}+(2 T)^{2}}-\frac{\left(1 / \tau_{e l}\right)^{2}}{\left(D p^{2}\right)^{2}+\left(1 / \tau_{e l}\right)^{2}}\right]\right. \\
\left.+\int_{2 T}^{1 / \tau_{e l}} \frac{d \omega}{2 \pi}\left[\frac{\omega t}{\left(D p^{2}\right)^{2}+\omega^{2}}+\frac{\omega\left(1-e^{-D p^{2} t}\right)}{D p^{2}\left(\left(D p^{2}\right)^{2}+\omega^{2}\right)}\right]\right\} .
\end{gathered}
$$

The last term is estimated by approximating $1-e^{-D p^{2} t} \approx D p^{2} t$ for $D p^{2} t<1$ and $1-e^{-D p^{2} t} \approx 1$ for $D p^{2} t>1$. Evaluating the integrals yields

$$
\begin{aligned}
& \Delta \Gamma(t) \approx \frac{1}{\nu_{1} \sqrt{D} \pi^{2}}\left[\sqrt{2} \pi t\left(\frac{1}{\sqrt{\tau_{e l}}}-\sqrt{2 T}\right)+4 \ln \left(\frac{1}{2 T \tau_{e l}}\right) \sqrt{t}\right. \\
& \left.+\frac{\pi}{2} \frac{1}{\sqrt{T}}+\mathcal{O}\left(\frac{1}{T \sqrt{t}}\right)\right] \quad \text { for } T t \gg 1 .
\end{aligned}
$$



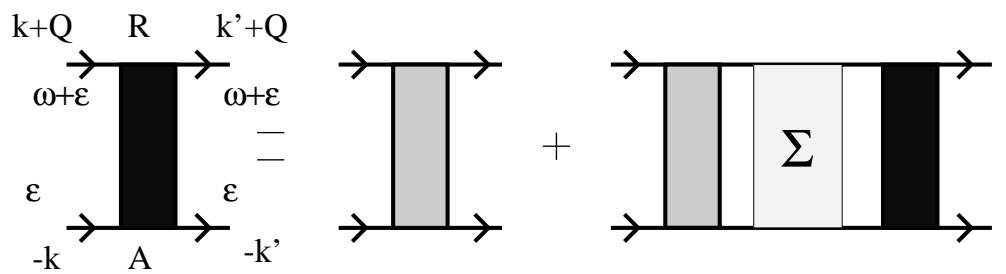

Figure 3.1: Bethe-Salpeter equation for the Cooperon without Vertex corrections. The non-interacting Cooperon $C_{n i}$ is defined in Fig.2.1(b) and Eq.(2.26). The interaction block $\Sigma$ does not contain Coulomb lines connecting the upper and lower propagator. As a consequence, the frequency is conserved at each electron line.

The prefactor can be expressed as $1 /\left(\nu_{1} \sqrt{D} \pi^{2}\right)=\sqrt{D} e^{2} /\left(\sigma_{1} \pi^{2}\right)$. Comparing this result with Eq.(30) of Ref. [38] (the term $\sim t^{3 / 2}$ comes of course from the contribution $|\omega|<2 T)$, one sees, that the dominant term, which is linear in $t$ and independent of temperature $T$, is exactly reproduced. This term would obviously lead to a constant dephasing time $1 / \tau_{\varphi}$ at $T=0$. The sub-leading terms are obtained within factors of order unity, due to the approximations made here. It is important to point out, that the term linear in $t$ arises from self-energy corrections. Thus vertex corrections are unimportant to obtain the leading contribution from $\omega>2 T$. On the other hand, without vertex corrections infinite orders of interaction corrections to the Cooperon can be easily resummed [30,29]. In this case the Bethe-Salpeter equation for the Cooperon $C_{w v}$ (wv: without vertex corrections) takes the form shown in Fig.3.1. All interactions are contained in the interaction block $\Sigma$. Neglecting vertex corrections means, that there are no Coulomb lines connecting the upper to the lower propagator. For example to first order in the RPA the diagrams shown in Figs.2.9(c),(d) are neglected, while Figs.2.9(a),(b) are included. Since the disorder is static, frequency is now conserved on each electron line. After averaging over disorder, the total momentum is conserved and the Cooperon depends only on the sum of the incoming momenta and the difference of the corresponding frequencies. Translating the diagrammatic expression for the Bethe-Salpeter equation in Fig.3.1 to an analytical equation yields

$$
\mathrm{C}_{w v}(\mathbf{Q}, \omega)=\mathrm{C}_{n i}(\mathbf{Q}, \omega)+\mathrm{C}_{n i}(\mathbf{Q}, \omega) \Sigma(\mathbf{Q}, \omega) \mathrm{C}_{w v}(\mathbf{Q}, \omega)
$$

where $\Sigma(\mathbf{Q}, \omega)$ is the interaction block, which has been evaluated to first order in the RPA in Refs. [30, 29, 31]. Eq.(3.62) is easily solved. Using the explicit form of the non-interacting Cooperon $C_{n i}$ (see Eq.(2.27)) in the limit $\mathcal{V} \rightarrow \infty$ one arrives 
at!

$$
\mathrm{C}_{w v}(\mathbf{Q}, \omega)=\frac{\Delta}{2 \pi \tau_{e l}^{2}} \frac{1}{D \mathbf{Q}^{2}-i \omega-\frac{\Delta}{2 \pi \tau_{e l}^{2}} \Sigma(\mathbf{Q}, \omega)}
$$

The corresponding dephasing rate for the Cooperon without vertex corrections is thus connected to the interaction block $\Sigma$ via

$$
\frac{1}{\tau_{w v}}=-\frac{\Delta}{2 \pi \tau_{e l}^{2}} \Sigma(0,0) \text {. }
$$

Note, that by solving the self-consistent Bethe-Salpeter equation one sums infinite orders in the interaction, even though the self-energy $\Sigma$ is only calculated to finite order. Since $\mathrm{C}_{w v}(\mathbf{Q}, \omega)=\int d t e^{i \omega t} \mathrm{C}_{w v}(\mathbf{Q}, t)$ with $\mathrm{C}_{w v}(\mathbf{Q}, t)=$ $\Theta(t)\left(\Delta /\left(2 \pi \tau_{e l}^{2}\right)\right) e^{-\left(D \mathbf{Q}^{2}+1 / \tau_{w v}\right) t}$ it is clear, that $-\Sigma(0,0)$ yields an exponential damping for the Fourier transformed Cooperon $\mathrm{C}_{w v}(\mathbf{Q}, t)$. To first order in the RPA $\Sigma(0,0)$ has been calculated by Fukuyama and Abrahams [29] and was rederived in Refs. [30, 31]. These calculations are fully quantum mechanical, since electronelectron interactions are included on a microscopic level and one expands in powers of the effective two-particle interaction. No mapping onto fluctuating external fields is needed. One finds [29, 30]

$$
\frac{1}{\tau_{w v}}=-2 \int \frac{d \mathbf{q} d \omega}{(2 \pi)^{d+1}} \operatorname{Im}\left(\frac{f_{\mathbf{q}}}{\epsilon(\mathbf{q}, \omega)}\right)\left[\operatorname{coth}\left(\frac{\omega}{2 T}\right)-\tanh \left(\frac{\omega}{2 T}\right)\right] \frac{D \mathbf{q}^{2}}{\left(D \mathbf{q}^{2}\right)^{2}+\omega^{2}}
$$

For $\omega<T$ the contribution from tanh can be neglected and one obtains the term from Eq.(3.44) which is linear in time. In contrast to Eq.(3.44), the equation for $1 / \tau_{w v}$ is divergent for small frequencies. To cure this singularity, $1 / \tau_{w v}$ is inserted self-consistently into the Diffuson propagator in Eq.(3.65). For the dephasing rate due to Nyquist noise $1 / \tau_{n n}$ the validity of such a procedure has been proven in this section. It will be seen in Sec.3.5, that $1 / \tau_{w v}$ coincides with the cutoff in the so called UCF-Diffuson. By applying the Eikonal method to the Diffuson it is shown in Sec.3.5, that the self-consistency procedure outlined above is also valid in that case. The most important insight gained from Eq.(3.65) is however, that the coth is canceled by tanh for frequencies larger than the temperature, $\omega>T$. The range of integration is thus indeed limited to $\omega<T$. It is emphasized, that one has summed infinite orders in the interaction and the cancellation occurs in every order. The central statement of Golubev and Zaikin is thus, that the second term containing

\footnotetext{
${ }^{1}$ Here the mesoscopic definition of the Cooperon is used which is normalized using the level spacing $\Delta$ instead of the density of states $\nu_{d}$. For macroscopic systems usually the latter normalization is used, since it has a finite thermodynamic limit [67]. In order not to introduce further notations, the mesoscopic definition is kept and only the cutoff $\Delta / \pi$ in the denominator of $C_{n i}$ is scaled to zero.
} 
tanh instead of coth, even though it appears in the same way as $\Delta \Gamma(t)$, i.e. in the exponent, does not contribute to dephasing.

In the opposite limit $T t \ll 1$ one finds that the dominant contribution to $\Delta \Gamma(t)$ comes from the frequency-regime $1 / t \leq \omega \leq 1 / \tau_{e l}$ where the oscillating terms can again be neglected. Thus the result for $T t \ll 1$ is obtained from Eq.(3.61) by replacing $2 T$ by $1 / t$. For $1 / t \ll 1 / \tau_{e l}$ this yields

$$
\Delta \Gamma(t) \approx \frac{1}{\nu_{1} \sqrt{D} \pi^{2}}\left[\frac{\sqrt{2} \pi}{\sqrt{\tau_{e l}}} t+4 \sqrt{t}\left(\ln \left(\frac{t}{\sqrt{2} \tau_{e l}}\right)+\mathcal{O}(1)\right)\right] \quad \text { for } T t \ll 1,
$$

in agreement with Ref. [38].

\section{b) $\varepsilon \gg T$}

For energies $\varepsilon$ much greater than the temperature $T$ it is justified to set $T=0$ and consequently $\operatorname{coth}(\omega /(2 T))=1$. The classical Nyquist noise contribution to the dephasing time is obtained by choosing $\omega_{c}=\varepsilon$. Now $1 / \tau_{n n}$ will be evaluated from Eq.(3.45) in the limit $\varepsilon \tau_{n n} \gg 1$. Again introducing the dimensionless variables $x:=D \mathbf{q}^{2} t$ and $y:=\omega t$, one obtains

$$
\begin{array}{r}
\Gamma(t)=\frac{2 K_{d} t^{1-d / 2}}{2 \pi \nu_{d} D^{d / 2}} \int_{0}^{\infty} d x \int_{0}^{\varepsilon t} d y x^{d / 2-1} y\left[\frac{1}{x^{2}+y^{2}}\left(1-\frac{\sin y}{y}\right)\right. \\
\left.+\frac{1}{x\left(x^{2}+y^{2}\right)}\left(\cos y\left[e^{-x} \frac{x^{2}-y^{2}}{x^{2}+y^{2}}+1\right]-\frac{x^{2}-y^{2}}{x^{2}+y^{2}}-e^{-x}-\frac{2 x y e^{-x} \sin y}{x^{2}+y^{2}}\right)\right] .
\end{array}
$$

The numerical constant $K_{d}$ is the same as in Eq.(3.47). Since $\tau_{n n}$ is determined from the condition $\Gamma\left(\tau_{n n}\right)=1$ only in the leading asymptotic behavior for $\varepsilon t \gg 1$ is of interest. The integrals are evaluated by approximating $e^{-x} \approx 1-x$ for $x<1$ and $e^{-x} \approx 0$ for $x>1$. The dominant contribution for $\varepsilon t \gg 1$ comes from the first term in the square braces in Eq.(3.67):

$$
\Gamma(t) \approx \frac{2 K_{d} t^{1-d / 2}}{2 \pi \nu_{d} D^{d / 2}} \int_{0}^{\infty} d x \int_{0}^{\varepsilon t} d y \frac{x^{d / 2-1} y}{x^{2}+y^{2}}\left(1-\frac{\sin y}{y}\right) .
$$

Calculating the integrals one arrives at

$$
\Gamma(t) \approx \frac{C_{d} \varepsilon^{d / 2}}{\nu_{d} D^{d / 2}} t
$$

with $C_{1}=\sqrt{2} / \pi, C_{2}=1 /(4 \pi)$ and $C_{3}=1 /\left(6 \pi^{2}\right)$. The dephasing rate for $T=0$ and $\varepsilon \tau_{n n} \gg 1$ is thus given by

$$
\frac{1}{\tau_{n n}}=\frac{C_{d}}{\nu_{d}}\left(\frac{\varepsilon}{D}\right)^{\frac{d}{2}}
$$


Formally this result resembles the inelastic quasiparticle damping due to electronelectron scattering $1 / \tau_{e e}\left(\xi_{\alpha}\right) \sim \nu_{d}^{-1}\left(\varepsilon_{\alpha} / D\right)^{d / 2}$ [20]. Here $\xi_{\alpha}$ is the quasiparticle energy. (Note that for finite frequencies $\varepsilon>0$ the external field $\mathbf{E}_{d r i}$ will in general also yield a contribution $1 / \tau_{A C}$ to the total dephasing rate $1 / \tau_{\varphi}=1 / \tau_{A C}+1 / \tau_{n n}$.) The result for three dimensions can be obtained from the corresponding dephasing rate at $\varepsilon=0$ and $T \tau_{n n} \gg 1$ by simply replacing $T$ by $\varepsilon$. For $d<3$ such a procedure fails. The reason for this is quite clear from looking at the formulas obtained in first order perturbation theory in the screened interaction given in Eq.(2.97). These equations where derived in the limit $\Gamma_{0} \gg 1 / \tau_{n n}$ but, as already mentioned, $\tau_{n n}$ can still be obtained for $\Gamma_{0} \rightarrow 0$ if $\Gamma_{0}$ is self-consistently replaced by $1 / \tau_{n n}$. Now it is clear, that for $T=0$ and $\varepsilon \gg 1$ both energy-scales $T$ and $\Gamma_{0}$ must be replaced by $\varepsilon$ times factors of order unity. (Note that in three dimensions the result is independent of $\left.\Gamma_{0}\right)$. This immediately yields the frequency dependence as given in Eq.(3.70). The same results were obtained from directly performing the perturbative calculations for finite $\varepsilon$ and $T=0$, see Eq.(2.99).

\subsubsection{The dephasing rate in finite systems}

For finite systems, two new energy scales enter the problem. First the average level spacing $\Delta$ and second the Thouless energy $E_{c}=D / L^{2}$. The results of the previous section were derived for infinite systems and are valid as long as the spectrum is effectively 'continuous' and the Thouless energy is smaller than the dephasing rate $1 / \tau_{\varphi}$. In the problem considered here, the spectrum can be viewed as 'continuous' if the dephasing rate is lager than the level spacing, $1 / \tau_{\varphi}>\Delta$. This condition is different from that for a continuous one particle energy spectrum, $\gamma_{q p}>\Delta$, where $\gamma_{q p}^{-1}$ is the inelastic (quasi-) particle life time. As already mentioned in Sec.2.1, this single particle time which is caused by inelastic processes is not the same as the dephasing time $\tau_{\varphi}$ to which also elastic processes like interactions with external magnetic fields do contribute. In the absence of any inelastic processes (i.e. if the one-particle spectrum is truely discrete) the Cooperon is still a well defined object, if the finite size cutoff $\Delta / \pi$ is introduced (see Eq.(2.27)). To obtain this result, non-perturbative methods are necessary [14]. It is thus clear, that one can not derive a dephasing time $1 / \tau_{\varphi}<\Delta$ from any calculation that performs a perturbative diagrammatic disorder averaging in terms of Cooperons. If one assumes, that at low temperatures the total dephasing rate is governed by the Nyquist noise contribution $1 / \tau_{\varphi} \approx 1 / \tau_{n n}$, Eq.(3.70) yields a simple condition for which frequencies $\varepsilon$ the spectrum is 'continuous'. For quasi $d$-dimensional systems with volume $V=L^{d} a^{3-d}$ the level-spacing $\Delta$ is connected to $\nu_{d}$ via $\nu_{d}=\nu a^{3-d}=a^{3-d} /(\Delta V)=1 /\left(\Delta L^{d}\right)$. Here $\nu=\nu_{3}$. The condition for a continuous spectrum now reads

$$
\Delta L^{d}\left(\frac{\varepsilon}{D}\right)^{\frac{d}{2}}>\Delta \Rightarrow \varepsilon^{d}>\left(\frac{D}{L^{2}}\right)^{d}=E_{c}^{d} .
$$


Thus in all dimensions the spectrum is continuous at $T=0$ as long as $\varepsilon>E_{c}$, i.e. for frequencies lager than the Thouless energy. For smaller frequencies the spectrum becomes discrete. In this regime other methods like the Random-MatrixTheory may have to be used to study disordered systems [30]. Eq.(3.71) was derived choosing the high frequency cutoff $\omega_{c}=\varepsilon$. Obviously the cutoff $\omega_{c}=1 / \tau_{e l}$ obtained by Golubev and Zaikin in Ref. [38] would yield a frequency independent dephasing rate. In this case $1 / \tau_{\varphi}$ will always be dominated by the zero-temperature value and thus it would follow that $1 / \tau_{\varphi}>\Delta$ independent of $\varepsilon$.

Mesoscopic systems are characterized by the condition $1 / \tau_{\varphi}<E_{c}$. In the following it is assumed, that the total dephasing rate is of the order $1 / \tau_{n n}$ and only quasi $d$-dimensional samples will be considered. As already mentioned, in this case Eq.(3.46) must be used to calculate the dephasing rate. Assuming periodic boundary conditions, the allowed momenta are given by the Cartesian coordinates $p_{i}=2 \pi n_{i} / L$ with $i=1, \cdots, d$. The dephasing rate is not sensitive to the choice of boundary conditions and different conditions will only lead to multiplicative factors of order unity [30]. Therefore for simplicity periodic boundary conditions are chosen. Since the $\mathbf{q}=\mathbf{0}$ mode is excluded from the sum in Eq.(3.46), the factor $D \mathbf{q}^{2}$ can not be smaller than the Thouless energy $E_{c}=D / L^{2}$, i.e. $D \mathbf{p}^{2} \geq E_{c}$. One is only interested in $\Gamma\left(t=\tau_{n n}\right)$ and thus for $\Delta<1 / \tau_{n n} \ll E_{c}$ the exponentially damped terms $\sim e^{-D \mathbf{p}^{2} t}$ in Eq.(3.46) can be safely ignored. Now $\Gamma(t)$ simplifies to

$$
\begin{array}{r}
\Gamma(t)=\frac{4}{\nu_{d}} \frac{1}{L^{d}} \sum_{\mathbf{q} \neq \mathbf{0}} \int_{0}^{\omega_{c}} \frac{d \omega}{2 \pi} \operatorname{coth}\left(\frac{\omega}{2 T}\right) \frac{\omega}{D \mathbf{q}^{2}}\left[\frac{D \mathbf{q}^{2}}{\left(D \mathbf{q}^{2}\right)^{2}+\omega^{2}}\left(t-\frac{\sin (\omega t)}{\omega}\right)+\right. \\
\left.-\frac{\left(D \mathbf{q}^{2}\right)^{2}-\omega^{2}}{\left[\left(D \mathbf{q}^{2}\right)^{2}+\omega^{2}\right]^{2}}+\frac{\cos (\omega t)}{\left(D \mathbf{q}^{2}\right)^{2}+\omega^{2}}\right] .
\end{array}
$$

The dominant contribution comes from the term which is linear in $t$. This implies, that in finite systems vertex corrections can be neglected in calculating $\tau_{n n}$. From $\Gamma\left(\tau_{n n}\right)=1$ one thus obtains

$$
\frac{1}{\tau_{n n}}=\frac{4}{\nu_{d}} \frac{1}{L^{d}} \sum_{\mathbf{q} \neq \mathbf{0}} \int_{0}^{\omega_{c}} \frac{d \omega}{2 \pi} \operatorname{coth}\left(\frac{\omega}{2 T}\right) \frac{\omega}{\left(D \mathbf{q}^{2}\right)^{2}+\omega^{2}} .
$$

For $T \tau_{n n} \gg 1, \varepsilon=0$ and $\omega_{c}=T$ this yields

$$
\frac{1}{\tau_{n n}}=\frac{4 T}{\pi \nu_{d}} \frac{1}{L^{d}} \sum_{\mathbf{q} \neq \mathbf{0}} \frac{1}{D \mathbf{q}^{2}} \arctan \frac{T}{D \mathbf{q}^{2}},
$$

and for $T=0, \varepsilon \tau_{n n} \gg 1$ with $\omega_{c}=\varepsilon$

$$
\frac{1}{\tau_{n n}}=\frac{1}{\pi \nu_{d}} \frac{1}{L^{d}} \sum_{\mathbf{q} \neq \mathbf{0}} \ln \left(1+\left[\frac{\varepsilon}{D \mathbf{q}^{2}}\right]^{2}\right) .
$$


These expressions have first been derived by means of a path-integral technique in Refs. [30,40]. Eq.(3.75) is precisely the expression derived from a phenomenological phase-uncertainty concept for the inverse quasiparticle dephasing time in Ref. [40]. In that work $\varepsilon$ is introduced as the quasiparticle energy and not as an external frequency. Formally it enters on the same level as a cutoff for the frequency integration. However, the relation between the dephasing time $1 / \tau_{\varphi}$, which is defined (and experimentally measured) as the cutoff in the weak localization correction and a single particle energy does not become quite clear. For $\varepsilon>E_{c}$ the sum in Eq.(3.75) can be transformed to an integral an the bulk result, derived in the previous section, is recovered. In this case the spectrum has been shown to be continuous. For $\varepsilon<E_{c}$ the system is effectively zero-dimensional with a discrete spectrum. As outlined above, since a non-perturbative calculation yields a finite size cutoff $\Delta / \pi$ for the Cooperon [14], one can not expect the above formalism to yield correct results in the limit $1 / \tau_{n n}<\Delta$. If nonetheless Eq. 3.75) is extrapolated to $\varepsilon<E_{c}$, the logarithm can be expanded to first order and one finds [40]

$$
\frac{1}{\tau_{n n}} \sim \Delta\left(\frac{\varepsilon}{E_{c}}\right)^{2} .
$$

Blanter [30] has pointed out, that this result is a priori uncontrolled and the Random Matrix Theory should be applied to study dephasing in systems with discrete spectrum. Using this technique he surprisingly obtained the same result as given in Eq.(3.76). The reason for this equivalence remains unclear [30].

At finite temperature and zero frequency the result for $d=1$ is [30]

$$
\frac{1}{\tau_{n n}} \sim \frac{T}{\left(k_{F} a\right)^{2}} \frac{L}{l}
$$

where $k_{F}$ is the Fermi-momentum and $l$ is the elastic mean free path. In this case the dephasing rate is lager than $\Delta$ as long as $T>l^{2} /\left(L^{2} \tau_{e l}\right)$.

\subsubsection{How first order perturbation theory is reproduced from the Eikonal approximation}

In Ref. [32] the effect of electron-electron interactions on the conductivity was studied in first order perturbation theory in the screened interaction. The result for the dephasing correction to weak localization is easily reproduced from the Eikonal approximation by expanding Eq.(3.35) to first order in the correlator $g(\mathbf{p}, \omega)$. This is equivalent to expanding $\exp (-\Gamma(\mathbf{k}, t))$ to first order; $\exp (-\Gamma(\mathbf{k}, t)) \approx 1-\Gamma(\mathbf{k}, t)$. Using Eq.(3.44), one obtains the corresponding first order correction to the conduc- 
tivity at $\varepsilon=0$ :

$$
\begin{aligned}
\sigma_{W L}^{(1)} & =\frac{2 \sigma_{d}}{\pi \nu_{d}} \operatorname{Re} \int \frac{d \mathbf{k}}{(2 \pi)^{2}} \int \frac{d \mathbf{q}}{(2 \pi)^{d}} \int_{-\infty}^{\infty} \frac{d \omega}{2 \pi} g(\mathbf{q}, \omega) \int_{0}^{\infty} d t e^{-\left(\Gamma_{0}+D \mathbf{k}^{2}\right) t}\left[\frac{t}{E_{\mathbf{k}}(\mathbf{q})-i \omega}\right. \\
& \left.+\frac{e^{-\left(E_{\mathbf{k}}(\mathbf{q})-i \omega\right) t}-1}{\left(E_{\mathbf{k}}(\mathbf{q})-i \omega\right)^{2}}-\frac{e^{-E_{\mathbf{k}}(\mathbf{q}) t}-e^{-i \omega t}}{i \omega\left(E_{\mathbf{k}}(\mathbf{q})-i \omega\right)}\right] .
\end{aligned}
$$

The integration over the time $t$ can be easily performed, resulting in

$$
\begin{aligned}
\sigma_{W L}^{(1)} & =\frac{2 \sigma_{d}}{\pi \nu_{d}} \operatorname{Re} \int \frac{d \mathbf{k}}{(2 \pi)^{2}} \int \frac{d \mathbf{q}}{(2 \pi)^{d}} \int_{-\infty}^{\infty} \frac{d \omega}{2 \pi} g(\mathbf{q}, \omega)\left[\frac{1}{\left(E_{\mathbf{k}}(\mathbf{q})-i \omega\right)\left(D \mathbf{k}^{2}+\Gamma_{0}\right)^{2}}\right. \\
& +\frac{1}{\left(E_{\mathbf{k}}(\mathbf{q})-i \omega\right)^{2}}\left(\frac{1}{E_{\mathbf{k}}(\mathbf{p})+D \mathbf{k}^{2}-i \omega+\Gamma_{0}}-\frac{1}{D \mathbf{k}^{2}+\Gamma_{0}}\right) \\
& \left.-\frac{1}{i \omega\left(E_{\mathbf{k}}(\mathbf{q})-i \omega\right)}\left(\frac{1}{E_{\mathbf{k}}(\mathbf{p})+D \mathbf{k}^{2}+\Gamma_{0}}-\frac{1}{D \mathbf{k}^{2}+i \omega+\Gamma_{0}}\right)\right] \\
& =\frac{2 \sigma_{d}}{\pi \nu_{d}} \operatorname{Re} \int \frac{d \mathbf{k}}{(2 \pi)^{2}} \int \frac{d \mathbf{q}}{(2 \pi)^{d}} \int_{-\infty}^{\infty} \frac{d \omega}{2 \pi} g(\mathbf{q}, \omega)\left[\frac{1}{\left(E_{\mathbf{k}}(\mathbf{q})-i \omega\right)\left(D \mathbf{k}^{2}+\Gamma_{0}\right)} \times\right. \\
& \left(\frac{1}{D \mathbf{k}^{2}+\Gamma_{0}}-\frac{1}{E_{\mathbf{k}}(\mathbf{p})+D \mathbf{k}^{2}-i \omega+\Gamma_{0}}\right) \\
& \left.+\frac{1}{i \omega\left(E_{\mathbf{k}}(\mathbf{p})+D \mathbf{k}^{2}+\Gamma_{0}\right)\left(D \mathbf{k}^{2}+i \omega+\Gamma_{0}\right)}\right] \\
& =\frac{2 \sigma_{d}}{\pi \nu_{d}} \int \frac{d \mathbf{k}}{(2 \pi)^{2}} \int \frac{d \mathbf{q}}{(2 \pi)^{d}} \int_{-\infty}^{\infty} \frac{d \omega}{2 \pi} g(\mathbf{q}, \omega)\left[\frac{1}{\left(D \mathbf{k}^{2}+\Gamma_{0}\right)^{2}\left(E_{\mathbf{k}}(\mathbf{p})+D \mathbf{k}^{2}-i \omega+\Gamma_{0}\right)}\right. \\
& \left.-\frac{1}{\left(E_{\mathbf{k}}(\mathbf{p})+D \mathbf{k}^{2}+\Gamma_{0}\right)\left|D \mathbf{k}^{2}-i \omega+\Gamma_{0}\right|^{2}}\right] .
\end{aligned}
$$

Now the definition for $g(\mathbf{q}, \omega)$ is inserted and $\omega_{c}=T$ is chosen. Approximating $\operatorname{coth}(\omega /(2 T)) \approx 2 T / \omega$ for $\omega<T$ and using $E_{\mathbf{k}}(\mathbf{q})+D \mathbf{k}^{2}=D(\mathbf{q}+\mathbf{k})^{2}$ one finally arrives at

$$
\begin{aligned}
\sigma_{W L}^{(1)} & =\frac{4 T \sigma_{d}}{\pi \nu_{d}^{2}} \int \frac{d \mathbf{k}}{(2 \pi)^{2}} \int \frac{d \mathbf{q}}{(2 \pi)^{d}} \int_{-T}^{T} \frac{d \omega}{2 \pi} \frac{1}{D \mathbf{q}^{2}}\left[\frac{1}{\left(D \mathbf{k}^{2}+\Gamma_{0}\right)^{2}\left(D(\mathbf{k}+\mathbf{p})^{2}-i \omega+\Gamma_{0}\right)}\right. \\
& \left.-\frac{1}{\left(D(\mathbf{k}+\mathbf{p})^{2}+\Gamma_{0}\right)\left|D \mathbf{k}^{2}-i \omega+\Gamma_{0}\right|^{2}}\right]
\end{aligned}
$$

This is just the result first obtained in Ref. [32] within diagrammatic perturbation theory. The details of the perturbative calculation were given in Sec.2.4. Using Eq. (2.92) and the approximation of Eq.(2.93) it is easy to see, that the general expression given in Eq.(2.87) reduces to Eq.(3.80) in the limit $\omega \rightarrow 0$. Now the first term in Eq.(3.80) arises from the first two terms in Eq.(3.78), while the second term in Eq.(3.80) is solely due to the third term of Eq.(3.78). In the diagrammatic theory this term comes from diagrams (c) and (d) in Fig.2.9, i.e. from vertex corrections. 
Recalling, that in the derivation this term was generated form the contribution $\sim \delta\left(\omega-\omega^{\prime}\right)$ (see remarks after Eq.(3.44)), it is now clear, that this part of the correlator in Eq.(3.29) indeed generates vertex corrections. The diagrams in Fig.2.9 are obtained by iterating the Bethe-Salpeter equation in Fig.2.4 to second order in the external field and connecting the potential lines. This connecting of external lines to a single interaction line is the diagrammatic equivalent of the averaging procedure described by Eq.(3.30) and Eq.(3.29). The part of the correlator $\sim \delta(\omega+$ $\left.\omega^{\prime}\right)$ 'connects' potential lines coupling to the same electron line, i.e. generates selfenergy diagrams, while $\delta\left(\omega-\omega^{\prime}\right)$ 'connects' lines from different Green's functions and thus generates vertex corrections.

\subsection{Dephasing due to external electric fields}

In this section it is assumed that the electrons are exposed to a classical potential of the form

$$
V(\mathbf{r}, t)=V_{0} \sin \left(\mathbf{q}_{0} \cdot \mathbf{r}-\omega_{0} t\right),
$$

where the wave-vector $\mathbf{q}_{0}$ and the frequency $\omega_{0}$ are fixed. According to Eq.(3.2) the corresponding longitudinal electric field is

$$
\mathbf{E}(\mathbf{r}, t)=\mathbf{E}_{0} \cos \left(\mathbf{q}_{0} \cdot \mathbf{r}-\omega_{0} t\right), \quad \mathbf{E}_{0}=\mathbf{q}_{0} V_{0} / e .
$$

For finite $\mathbf{q}_{0}$ the electric field leads to a modulation of the charge density $\rho(\mathbf{r}, t)=$ $\frac{1}{4 \pi} \nabla \cdot \mathbf{E}(\mathbf{r}, t)$

$$
\begin{gathered}
\rho(\mathbf{r}, t)=\rho_{0} \sin \left(\mathbf{q}_{0} \cdot \mathbf{r}-\omega_{0} t\right), \\
\rho_{0}=-\frac{\mathbf{q}_{0} \cdot \mathbf{E}_{0}}{4 \pi}=-\frac{q_{0}^{2} V_{0}}{4 \pi e} .
\end{gathered}
$$

It is emphasized that $\mathbf{E}(\mathbf{r}, t)$ is the total screened potential, which is the sum of the external potential and the induced potential. Of course, the Maxwell equation inside a metal contains a dissipative term, so that the field inside the metal is not given by a simple propagating wave [41]. In general one expects that the field distribution inside the metal depends on the boundary conditions and on the precise manner in which the microwaves are coupled into the system. Such a calculation is beyond the scope of this work. However, if the spatial variation of the field is sufficiently slow (i.e. $q_{0}$ is sufficiently small) the field inside the metal can still be approximated by a plane wave. To estimate the upper limit for $q_{0}$ where this approximation is correct, it is assumed that the external potential applied to the electrons is $V^{e x t}(\mathbf{r}, t)=V_{0}^{e x t} \sin \left(\mathbf{q}_{0} \cdot \mathbf{r}-\omega_{0} t\right)$. The total potential is then

$$
V(\mathbf{r}, t)=V_{0}^{e x t} \operatorname{Im}\left[\epsilon^{-1}\left(\mathbf{q}_{0}, \omega_{0}\right) e^{i\left(\mathbf{q}_{0} \cdot \mathbf{r}-\omega_{0} t\right)}\right],
$$


where the longitudinal electric function in the diffusive regime is given in Eq.(2.82). Screening can be ignored in the regime where $\epsilon\left(\mathbf{q}_{0}, \omega_{0}\right)$ can be approximated by unity. From Eq.(2.82) it is easy to see that in $d=1$ this is the case when

$$
q_{0} \ll \sqrt{\frac{\omega_{0}}{D}},
$$

while in $d=2$ the external field is effectively not screened if

$$
q_{0} \ll \frac{\omega_{0}}{2 \pi e^{2} \nu_{2} D} .
$$

The semiclassical approximation requires $q_{0}<1 / l$ and $\left|\omega_{0}\right|<1 / \tau_{e l}$ so that in metals with $2 \pi e^{2} \nu_{2}>1 / l$ the above inequalities Eqs.(3.86), (3.87) for $q_{0}$ are more restrictive. It is assumed that they are satisfied and the potential $V(\mathbf{r}, t)$ is indeed given by Eq.(3.81). If one takes the limit $\mathbf{q}_{0} \rightarrow 0$ keeping $\mathbf{E}_{0}$ finite, a time-dependent but spatially constant electric field is obtained. Then there is no charge density fluctuation. This case has been solved exactly by Altshuler, Aronov, and Khmelnitskii [22], and it will be shown that the Eikonal method reproduces their exact solution. Moreover, also the case of finite $\mathbf{q}_{0}$, which has not been discussed previously, will be investigated. It is found that in this case there exists a parameter regime where the dephasing rate is proportional to the square root of the power absorbed by the sample, in agreement with the experiment 42].

In the presence of an external space- and time-dependent field the longitudinal conductivity tensor is not diagonal in frequency space, and the weak localization correction is given by Eq.(3.25). In the absence of fluctuating fields the Cooperon does not depend on $t_{0}$, so that Eq.(3.25) reduces to

$$
\delta \sigma_{W L}\left(\varepsilon, \varepsilon^{\prime}\right)=2 \pi \delta\left(\varepsilon-\varepsilon^{\prime}\right) \delta \sigma_{W L}(\varepsilon),
$$

where $\delta \sigma_{W L}(\varepsilon)$ is given in Eq.(3.32). For a static driving field $\mathbf{E}_{d r i}\left(\varepsilon^{\prime}\right)=2 \pi \delta\left(\varepsilon^{\prime}\right) \mathbf{E}_{d r i}$ Eq.(3.24) now yields

$$
\delta \mathbf{j}_{W L}(\varepsilon)=\delta \sigma_{W L}(\varepsilon, 0) \mathbf{E}_{d r i}=2 \pi \delta(\varepsilon) \delta \sigma_{W L}(\varepsilon) \mathbf{E}_{d r i} .
$$

Consequently the static conductivity is in this case defined as

$$
\delta \sigma_{W L}:=\lim _{\varepsilon \rightarrow 0} \delta \sigma_{W L}(\varepsilon)
$$

To obtain an expression, which in the presence of fluctuating non-equilibrium fields is analog to $\delta \sigma_{W L}$, one uses the definition given in Eq.(3.90) together with

$$
2 \pi \delta(\varepsilon) \delta \sigma_{W L}(\varepsilon)=\delta \sigma_{W L}(\varepsilon, 0) .
$$

The resulting expression for the static conductivity can be written as

$$
\delta \sigma_{W L}=-\frac{\sigma_{d}}{\pi \nu_{d}} \int_{\tau_{e l}}^{\infty} d t \lim _{T_{0} \rightarrow \infty} \frac{1}{2 T_{0}} \int_{-T_{0}}^{T_{0}} d t_{0} \mathcal{C}\left(\mathbf{r}, \mathbf{r} ; \frac{t}{2},-\frac{t}{2}, t_{0}\right) .
$$


To see this, the $\delta$-function is represented as

$$
2 \pi \delta(\varepsilon)=\lim _{T_{0} \rightarrow \infty} \int_{-T_{0}}^{T_{0}} d \tau e^{i \varepsilon \tau}
$$

This expression is inserted into Eqs.(3.91), (3.25) and the limit $\varepsilon \rightarrow 0$ is taken before $T_{0} \rightarrow \infty$. Following Ref. [22], Eq.(3.92) will be used to define the dephasing rate due to non-equilibrium fields.

\subsubsection{Uniform electric field}

Taking in Eq.(3.81) the limit $\mathbf{q}_{0} \rightarrow 0$ keeping $\mathbf{E}_{0}=\mathbf{q}_{0} V_{0} / e$ constant, one obtains the spatially constant electric field, $\mathbf{E}(t)=\mathbf{E}_{0} \cos \left(\omega_{0} t\right)$. As already mentioned, this case has been solved exactly by Altshuler et al. [22], who worked in a gauge where the electric field is represented in terms of a vector potential. In contrast, the Eikonal approach represents the electric field in terms of a scalar potential. Of course, the same result for physical observables should be obtained in any gauge. It will now be shown how to recover the dephasing rate calculated in Ref. [22] within the Eikonal method.

From Eq.(3.81) one easily obtains the Fourier coefficients $V(\mathbf{q}, \omega)$ of the potential $V$. Inserting the result into the expression for $V_{t_{0}}(\mathbf{q}, \omega)$ given in Eq.(3.18) yields

$$
\begin{aligned}
V_{t_{0}}(\mathbf{q}, \omega) & =\frac{V_{0}}{2}(2 \pi)^{d+1}\left[\delta\left(\omega-\omega_{0}\right)-\delta\left(\omega+\omega_{0}\right)\right] \\
& \times\left[e^{-i \omega_{0} t_{0}} \delta\left(\mathbf{q}-\mathbf{q}_{0}\right)+e^{i \omega_{0} t_{0}} \delta\left(\mathbf{q}+\mathbf{q}_{0}\right)\right] .
\end{aligned}
$$

Substituting this expression into Eqs.(3.16), (3.20), performing the integrations, and finally taking the limit $\mathbf{q}_{0} \rightarrow 0$ keeping $\mathbf{E}_{0}=\mathbf{q}_{0} V_{0} / e$ constant one obtains for the first order term in the Eikonal expansion

$$
F_{1}(\mathbf{k}, t ; t / 2)=2 D \mathbf{k} \cdot \mathbf{p}(t) t \sin \left(\omega_{0} t_{0}\right)
$$

where

$$
\mathbf{p}(t)=\frac{2 e \mathbf{E}_{0}}{\omega_{0}}\left[\cos \left(\omega_{0} t / 2\right)-2 \frac{\sin \left(\omega_{0} t / 2\right)}{\omega_{0} t}\right]
$$

The term quadratic in the field is

$$
F_{2}(t ; t / 2)=4 \frac{D\left(e E_{0}\right)^{2} t}{\omega_{0}^{2}}\left[1+\frac{1}{2} \cos \left(\omega_{0} t\right)-\frac{3}{2} \frac{\sin \left(\omega_{0} t\right)}{\omega_{0} t}\right] \sin ^{2}\left(\omega_{0} t_{0}\right) .
$$

Because $F_{1}$ is independent of $\mathbf{r}$, and $F_{2}$ is independent of $\mathbf{k}$ and $\mathbf{r}$ these labels where omitted. By construction, the higher order terms $F_{n}, n \geq 3$ involve more than two gradients, which give rise to at least an additional power of $\mathbf{q}_{0}$. It follows that for 
$\mathbf{q}_{0} \rightarrow 0$ the Eikonal expansion truncates at the second order. Hence in this limit the exact Eikonal factor $F$ in Eq.(3.22) is simply given by

$$
F(\mathbf{k}, t ; t / 2)=2 D \mathbf{k} \cdot \mathbf{p}(t) t \sin \left(\omega_{0} t_{0}\right)+F_{2}(t ; t / 2) .
$$

The Cooperon at coinciding space points is thus given by

$$
\begin{aligned}
\mathcal{C}\left(\mathbf{r}, \mathbf{r} ; \frac{t}{2},-\frac{t}{2}, t_{0}\right) & =\Theta(t) \int \frac{d \mathbf{k}}{(2 \pi)^{d}} e^{-\left(\Gamma_{0}+D \mathbf{k}^{2}\right) t} e^{-F(\mathbf{k}, t ; t / 2)} \\
& =\frac{\Theta(t)}{(4 \pi D t)^{d / 2}} e^{-\Gamma_{0} t-\Gamma\left(t, t_{0}\right)}
\end{aligned}
$$

with

$$
\Gamma\left(t, t_{0}\right)=-D \mathbf{p}^{2}(t) t \sin ^{2}\left(\omega_{0} t_{0}\right)+F_{2}(t ; t / 2) .
$$

Using Eqs.(3.96) and (3.97) one obtains

$$
\Gamma\left(t, t_{0}\right)=\alpha f\left(\omega_{0} t\right)\left[1-\cos \left(2 \omega_{0} t_{0}\right)\right]
$$

where the dimensionless parameter $\alpha$ is

$$
\alpha=\frac{D\left(e E_{0}\right)^{2}}{\omega_{0}^{3}}
$$

and

$$
f(x)=x+\sin (x)-\frac{8}{x} \sin ^{2}\left(\frac{x}{2}\right) .
$$

To identify the dephasing rate $1 / \tau_{A C}$ due to the time-dependent electric field the observable $\delta \sigma_{W L}$ defined in Eq.(3.92) is calculated. The $t_{0}$-integration can be expressed in terms of the Bessel-function $I_{0}(z)$,

$$
\begin{aligned}
\lim _{T_{0} \rightarrow \infty} \frac{1}{2 T_{0}} \int_{-T_{0}}^{T_{0}} d t_{0} e^{\alpha f \cos \left(2 \omega_{0} t_{0}\right)} \\
\quad=\frac{1}{\pi} \int_{0}^{\pi} d \varphi e^{\alpha f \cos \varphi}=I_{0}(\alpha f)
\end{aligned}
$$

Introducing the dimensionless integration variable $x=\omega_{0} t$ and defining

$$
\gamma=\Gamma_{0} / \omega_{0}
$$

the final result can be written as

$$
\delta \sigma_{W L}=-\frac{\sigma_{d}}{\pi \nu_{d}} \frac{\omega_{0}^{\frac{d}{2}-1}}{(4 \pi D)^{d / 2}} \int_{\omega_{0} \tau_{e l}}^{\infty} \frac{d x}{x^{d / 2}} e^{-\alpha f(x)-\gamma x} I_{0}(\alpha f(x)) .
$$


Apart from a different prefactor this expression agrees with Eq.(5) of Ref. [22]. (Note that the $\alpha$ defined here is only half as large as the parameter $\alpha$ introduced in Ref. [22], and the integration variable used here is twice as large as the corresponding $x$ in Ref. [22]. The origin for these factors of two lies in the fact that in equation (3.1) for the Cooperon the external potential enters with the time-arguments $t_{0} \pm t$, and not with $t_{0} \pm t / 2$, as incorrectly assumed in Ref. [22], see also Ref. [15].) In the absence of microwaves, i.e. for $\alpha=0$, the term $e^{-\gamma x}$ is needed to enforce convergence of the integral (3.106) in $d \leq 2$. On the other hand, for finite $\alpha$ the integral is finite even if one sets $\gamma=0$. For $\alpha \ll 1$ and $\gamma \ll 1$ the integral is cut off at $x \gg 1$, so that one may approximate $f(x) \approx x$. The characteristic cutoff energy can then be identified with the dephasing rate due to the microwave field. In the regime $\gamma \ll \alpha \ll 1$ one obtains

$$
\frac{1}{\tau_{A C}}=\omega_{0} \alpha=\frac{D\left(e E_{0}\right)^{2}}{\omega_{0}^{2}} .
$$

On the other hand, for $\alpha \gg 1$ the $x$-integration is cut off at $x \ll 1$, so that one may approximate $f(x)$ by its leading term for small $x$, which is given by $f(x) \approx \frac{x^{5}}{3 \times 5 !}=$ $\frac{x^{5}}{360}$. The dephasing rate is then

$$
\frac{1}{\tau_{A C}}=\omega_{0}\left(\frac{\alpha}{360}\right)^{1 / 5} \approx 3.2 \omega_{0}^{2 / 5}\left[D\left(e E_{0}\right)^{2}\right]^{1 / 5} .
$$

This expression is valid in the regime

$$
\alpha \gg \max \left\{1,360 \gamma^{5}\right\},
$$

where $1 / \tau_{A C} \gg \Gamma_{0}$. The explicit results for the correction $\delta \sigma_{W L}$ to the conductivity in $d=1$ and $d=2$ are given in Ref. [22].

For a comparison with experiments [42, 44] it is useful to rewrite the above expressions in terms of the microwave-power $P$ coupled into the sample, which can be estimated as 44, 44]

$$
P=\frac{\left(E_{0} L\right)^{2}}{2 R_{t o t}}
$$

Here $L$ is the effective sample length including the leads, and $R_{t o t}$ is the total resistance. The dimensionless parameter $\alpha$ in Eq.(3.102) can then be written as

$$
\alpha=\frac{E_{c}}{\omega_{0}} \frac{2 e^{2} R_{t o t} P}{\omega_{0}^{2}},
$$

where $E_{c}=D / L^{2}$ is the Thouless energy. For $\gamma \ll \alpha \ll 1$ the dephasing rate can be written as

$$
\frac{1}{\tau_{A C}}=E_{c} \frac{2 e^{2} R_{t o t} P}{\omega_{0}^{2}}
$$


and for $\alpha \gg \max \left\{1,360 \gamma^{5}\right\}$ Eq.(3.108) predicts

$$
\frac{1}{\tau_{A C}}=3.2 E_{c}^{1 / 5} \omega_{0}^{2 / 5}\left[2 e^{2} R_{t o t} P\right]^{1 / 5} .
$$

According to Eqs.(3.111)-(3.113) for sufficiently small microwave power $P$ the dephasing rate should grow linear with $P$, and eventually cross over to a $P^{1 / 5}$ law. Furthermore, in the linear regime $1 / \tau_{A C}$ should decrease as $1 / \omega_{0}^{2}$ as the frequency increases. This is not what has been observed in the experiment by Wang and Lindelof [42]. As pointed out by these authors, at smaller frequencies $1 / \tau_{A C}$ is proportional to $P^{1 / 2}$. Furthermore, the data shown in Fig.3 of Ref. [42] indicate that at constant microwave power $1 / \tau_{A C} \propto \omega_{0}^{-1}$. In the following section a simple explanation of these experimental facts shall be proposed.

\subsubsection{Dephasing due to longitudinal electric fields with fi- nite wave-length}

In the previous section the limit $q_{0} \rightarrow 0$ was taken, which is justified if the wavelength $\lambda=2 \pi / q_{0}$ of the microwave is much larger than the size $L$ of the sample. A precise estimate of $q_{0}$ is difficult, since it depends on the shape of the experimental set up, the dispersion relation of microwaves in the sample and the way the microwaves are coupled to the probe. However, it is not unreasonable to assume, that the wavelength will be of the order of the samples extension, i.e. that the dimensionless parameter $q_{0} L$ is of the order of unity. It is therefore important to know the corrections to the results of Sec.3.4.1 for finite $q_{0} L$. Now the leading correction to the dephasing rate due to longitudinal fluctuating electric microwave fields for small but finite $q_{0} L$ in the limit of weak microwave power will be calculated.

Substituting the Fourier representation (3.94) of the microwave potential into Eq.(3.16), performing the integrations, and expanding the resulting expression to first order in $\mathbf{q}_{0}$, one obtains

$$
\begin{aligned}
F_{1}(\mathbf{k}, t ; \mathbf{r}, t / 2) & =2 D \mathbf{k} \cdot \mathbf{p}(t) t\left[\sin \left(\omega_{0} t_{0}\right)-\left(\mathbf{q}_{0} \cdot \mathbf{r}\right) \cos \left(\omega_{0} t_{0}\right)\right] \\
& +i \beta g\left(\omega_{0} t\right) \cos \left(\omega_{0} t_{0}\right)\left[1-2 D\left(\hat{\mathbf{q}}_{0} \cdot \mathbf{k}\right)^{2} t\right] \\
& +O\left(E_{0} q_{0}^{2}\right)
\end{aligned}
$$

Here

$$
g(x)=x \cos \left(\frac{x}{2}\right)-2 \sin \left(\frac{x}{2}\right)
$$

the dimensionless parameter $\beta$ is given by

$$
\beta=\frac{2 D e E_{0} q_{0}}{\omega_{0}^{2}},
$$


and $\hat{\mathbf{q}}_{0}=\mathbf{q}_{0} / q_{0}$ is a unit vector in the direction of $\mathbf{q}_{0}$. Note that $\beta$ can be expressed in terms of the microwave power $P$ (see Eq.(3.110)) and the Thouless energy $E_{c}$ as follows,

$$
\beta=2 q_{0} L \frac{E_{c}}{\omega_{0}} \frac{\sqrt{2 e^{2} R_{t o t} P}}{\omega_{0}} .
$$

From Eq.(3.15) one sees, that $F_{2}$ is connected to $F_{1}$ by

$$
F_{2}(\mathbf{k}, \tau ; \mathbf{r}, t)=-\int_{0}^{\tau} d \tau^{\prime} e^{\left(D \hat{\mathbf{P}}_{\mathbf{r}}^{2}+2 D \mathbf{k} \cdot \hat{\mathbf{P}}_{\mathbf{r}}+\partial_{t}\right)\left(\tau^{\prime}-\tau\right)} D\left[\hat{\mathbf{P}}_{\mathbf{r}} F_{1}\left(\mathbf{k}, \tau^{\prime} ; \mathbf{r}, t\right)\right]^{2} .
$$

Inserting Eq.(3.114) yields

$$
\begin{aligned}
F_{2}(\mathbf{k}, t ; \mathbf{r}, t / 2) & =4 D\left(e E_{0}\right)^{2} \omega_{0}^{-3} u\left(\omega_{0} t\right) \sin ^{2}\left(\omega_{0} t_{0}\right) \\
& +O\left(E_{0}^{2} q_{0}\right)
\end{aligned}
$$

with

$$
u(x)=x+\frac{x}{2} \cos (x)-\frac{3}{2} \sin (x)
$$

Ignoring corrections of order $E_{0} q_{0}^{2}$ and $E_{0}^{2} q_{0}$ to the Eikonal factor $F$, one obtains for the Cooperon at coinciding space points

$$
\mathcal{C}\left(\mathbf{r}, \mathbf{r} ; \frac{t}{2},-\frac{t}{2}, t_{0}\right)=\frac{\Theta(t)}{(4 \pi D t)^{d / 2}} e^{-\Gamma_{0} t-\Gamma\left(t, t_{0}\right)} \frac{e^{-i \beta g\left(\omega_{0} t\right) \cos \left(\omega_{0} t_{0}\right)}}{\sqrt{1-2 i \beta g\left(\omega_{0} t\right) \cos \left(\omega_{0} t_{0}\right)}}
$$

Here $\Gamma\left(t, t_{0}\right)$ is the contribution to the dephasing rate for microwaves with infinite wavelength (see Eq.(3.101)), and the last factor in Eq.(3.121) is the leading correction for finite $\mathbf{q}_{0}$. Using Eq.(3.92) and (3.100) and introducing again the dimensionless integration variable $x=\omega_{0} t$, one finally obtains for the weak localization correction to the static conductivity

$$
\delta \sigma_{W L}=-\frac{\sigma_{d}}{\pi \nu_{d}} \frac{\omega_{0}^{\frac{d}{2}-1}}{(4 \pi D)^{d / 2}} G_{d}(\alpha, \beta, \gamma)
$$

where the dimensionless real function $G_{d}(\alpha, \beta, \gamma)$ is given by

$$
G_{d}(\alpha, \beta, \gamma)=\frac{1}{\pi} \int_{\omega_{0} \tau_{e l}}^{\infty} \frac{d x}{x^{d / 2}} e^{-\alpha f(x)-\gamma x} \int_{0}^{\pi} d \varphi \frac{e^{\alpha f(x) \cos (2 \varphi)-i \beta g(x) \cos \varphi}}{\sqrt{1-2 i \beta g(x) \cos \varphi}}
$$

If one sets $\beta=0$ from Eq.(3.122) the result (3.106) for $\mathbf{q}_{0}=0$ is recovered. In general the relevant long-time cutoff in Eq.(3.122) depends on the relative order of magnitude of the three dimensionless parameters $\alpha, \beta$, and $\gamma$. Keeping in mind that $\alpha \propto E_{0}^{2}$ and $\beta \propto q_{0} E_{0}$, and assuming that at low temperatures the phenomenological 
parameter $\gamma$ is negligible, it is clear that for sufficiently weak microwave power the relevant cutoff scale is set by the parameter $\beta$. Note that according to Eqs.(3.102) and (3.116)

$$
\frac{\beta}{\alpha}=\frac{2 q_{0} \omega_{0}}{e E_{0}}=\frac{2 q_{0} L \omega_{0}}{\sqrt{2 e^{2} R_{t o t} P}}
$$

Hence, for sufficiently small microwave power $P$ the ratio $\beta / \alpha$ becomes always larger than unity. In this regime it is justified to set $\alpha=\gamma=0$ in Eq.(3.122), so that one needs to calculate

$$
G_{d}(\beta)=\frac{1}{\pi} \int_{\omega_{0} \tau_{e l}}^{\infty} \frac{d x}{x^{d / 2}} \int_{0}^{\pi} d \varphi \frac{e^{-i \beta g(x) \cos \varphi}}{\sqrt{1-2 i \beta g(x) \cos \varphi}} .
$$

Note that $G_{d}(0)=\infty$ in $d \leq 2$, which is due to the slow decay of the integral for large $x$. On the other hand, for any finite $\beta$ the $x$-integration is cut off, so that the integral exists. As in Sec.3.4.1 the energy scale where the integration is cut off is identified with the dephasing rate. The standard weak localization correction is obtained from Eq.(3.122) by setting $\alpha=\beta=0$ and identifying $1 / \tau_{\varphi}=\omega_{0} \gamma=\Gamma_{0}$. To leading order this yields the well known result

$$
\delta \sigma_{W L}=-\frac{\sigma_{d}}{\pi \nu_{d}} \frac{\omega_{0}^{\frac{d}{2}-1}}{(4 \pi D)^{d / 2}} \int_{\omega_{0} \tau_{e l}}^{\infty} \frac{d x}{x^{d / 2}} e^{-\gamma x} \approx-\frac{\sigma_{d}}{\pi \nu_{d}} \frac{\omega_{0}^{\frac{d}{2}-1}}{(4 \pi D)^{d / 2}} \int_{\omega_{0} \tau_{e l}}^{\omega_{0} \tau_{\varphi}} \frac{d x}{x^{d / 2}} .
$$

The $x$-integration is effectively cut off at $x_{c}(\beta=0)=\omega_{0} \tau_{\varphi}$. In the presence of fluctuating external fields with parameters $\beta \gg \alpha, \gamma$, the integral in Eq.(3.125) will be effectively cut off at $x_{c}(\beta) \ll x_{c}(0)$. Consequently the dephasing time due to microwave radiation $\tau_{A C}$ is in this case defined as $\tau_{A C}=x_{c}(\beta) / \omega_{0}$. For $\beta g(x) \ll 1$ the integrand in Eq.(3.125) behaves like $1 / x^{d / 2}$. The integrand starts to oscillate at $|\beta g(x)| \approx 1$. To obtain an order of magnitude estimate for $x_{c}$ one may thus derive this value from the condition $\left|\beta g\left(x_{c}\right)\right| \approx 1$. For $\beta \ll 1$ this yields

$$
\frac{1}{\tau_{A C}} \propto \omega_{0} \beta=\frac{2 D e E_{0} q_{0}}{\omega_{0}}=2 q_{0} L E_{c} \frac{\sqrt{2 e^{2} R_{t o t} P}}{\omega_{0}} .
$$

On the other hand, for $\beta \gg 1$ the $x$-integration is cut off at small $x$. In this case one may approximate $g(x) \approx-\frac{x^{3}}{12}$ and obtains

$$
\frac{1}{\tau_{A C}} \propto \omega_{0} \beta^{1 / 3}=\left(2 q_{0} L\right)^{1 / 3} E_{c}^{1 / 3} \omega_{0}^{1 / 3}\left[2 e^{2} R_{t o t} P\right]^{1 / 6} .
$$

To numerically test the above estimates, the function

$$
\tilde{G}_{d}\left(\beta, x_{0}\right):=\frac{1}{\pi} \int_{\omega_{0} \tau_{e l}}^{x_{0}} \frac{d x}{x^{d / 2}} \int_{0}^{\pi} d \varphi \frac{e^{-i \beta g(x) \cos \varphi}}{\sqrt{1-2 i \beta g(x) \cos \varphi}} .
$$




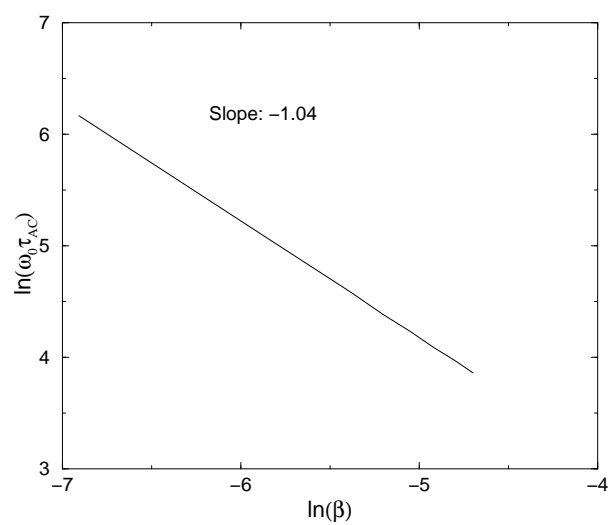

Figure 3.2: $\ln \left(\omega_{0} \tau_{A C}\right)$ as function of $\ln (\beta)$ in $d=2$ for $0.001 \leq \beta \leq 0.01$. The lower cutoff is $\omega_{0} \tau_{\text {el }}=0.01$. Here $\tau_{A C}$ is identified as $\tau_{A C}=x_{c} / \omega_{0}$, where $x_{c}$ is the smallest value for $x_{0}$ with $\left|\left(1 / \tilde{G}_{d}\left(\beta, x_{0}\right)\right)\left[\tilde{G}_{d}\left(\beta, x_{0}\right)-\ln \left(x_{0} /\left(\omega_{0} \tau_{\text {el }}\right)\right)\right]\right|>0.01$.

is introduced. Due to the rapid oscillation of the integrand for large $x$ a controlled computation of the limit $x_{0} \rightarrow \infty$ is rather difficult. Numerical evaluation of $\tilde{G}_{d}\left(\beta, x_{0}\right)$ as a function of $x_{0}$ shows, that the dominant contribution to the integrals comes indeed from the region where the integrand can be well approximated by $1 / x^{d / 2}$. In accordance with Eq.(3.126) one can thus extract $\tau_{A C}$ from the maximal value of $x_{0}$ for which the condition

$$
\tilde{G}_{d}\left(\beta, x_{0}\right) \approx \int_{\omega_{0} \tau_{e l}}^{x_{0}} \frac{d x}{x^{d / 2}}
$$

is still satisfied. Here only the results for $d=2$ will be presented, where condition (3.130) reads

$$
\tilde{G}_{d}\left(\beta, x_{0}\right) \approx \ln \left(\frac{x_{0}}{\omega_{0} \tau_{e l}}\right)
$$

and $x_{c}=\omega_{0} \tau_{A C}$ was determined as that value of $x_{0}$, where $\tilde{G}_{d}\left(\beta, x_{0}\right)$ deviates from $\ln \left(x_{0} /\left(\omega_{0} \tau_{e l}\right)\right)$ by more than one percent. The lower cutoff in Eq.(3.129) was chosen as $\omega \tau_{e l}=0.01$. From Eqs.(3.127), (3.128) it is expected that $\ln \left(\omega_{0} \tau_{A C}\right)=-\ln (\beta)$ for $\beta \ll 1$ and $\ln \left(\omega_{0} \tau_{A C}\right)=-(1 / 3) \ln (\beta)$ for $\beta \gg 1$. The corresponding numerical results are shown in Fig. 3.2 and(3.3). They are in good agreement with the analytical estimates. Hence, for sufficiently small $P$ the dephasing rate $1 / \tau_{A C}$ is proportional to $P^{1 / 2}$. Moreover, in this regime $\omega_{0} / \tau_{A C}$ should be independent of $\omega_{0}$ as long as the dispersion of the longitudinal density wave (i.e. the dependence of $q_{0}$ on $\omega_{0}$ ) can be neglected. Note that the condition $\beta \gg \alpha \gg \gamma$ where $1 / \tau_{A C}$ should exhibit a $P^{1 / 2}$-dependence can also be written as

$$
D q_{0}^{2} \gg \frac{\omega_{0}}{V_{0}} \Gamma_{0} \gg \Gamma_{0}
$$




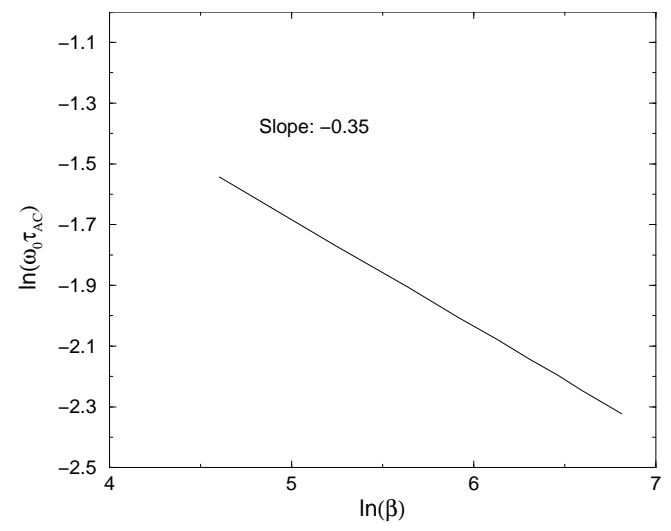

Figure 3.3: $\ln \left(\omega_{0} \tau_{A C}\right)$ as function of $\ln (\beta)$ in $d=2$ for $100 \leq \beta \leq 1000$. The lower cutoff is $\omega_{0} \tau_{e l}=0.01$. $\tau_{A C}$ is identified as in Fig. 3.2.

Thus, for finite $\Gamma_{0}$ the value of $q_{0}$ must be sufficiently large to observe the $P^{1 / 2}$-law . Keeping in mind that according to the conventional point of view [32, 43, 22] the intrinsic dephasing rate $\Gamma_{0}$ should vanish for $T \rightarrow 0$, it is concluded that at sufficiently low temperatures and small $V_{0}$ Eq.(3.132) can be satisfied for experimentally relevant wave-vectors $q_{0}$. Note that according to Ref. [43] the observed saturation of the dephasing time, which is typically of the order of a few nanoseconds, is due to some external noise. If this is correct, then the experimentally observed saturation value of the dephasing rate should not be identified with $\Gamma_{0}$. The same arguments apply for the condition $\left|V_{0}\right| \gg \Gamma_{0}$ that follows from Eq.(3.132) together with Eqs.(3.86),(3.87). For low enough temperatures this inequality should always be satisfied. (Newest experimental data [45] indicate $\Gamma_{0} \rightarrow 0$ for $T \rightarrow 0$ in narrow Ag wires. These probes therefore seem to be suiting to test the findings of this section.) In conclusion, in the limit $T \rightarrow 0$, the only relevant restriction following from Eq.(3.132) should be $\omega_{0} \gg V_{0}$.

The results for the dephasing time due to external microwave radiation and the assumptions made to obtain them can be summarized as follows: First of all it is assumed that the microwave field inside the metal can be approximated by a propagating wave with wave-vector $\mathbf{q}_{0}$, frequency $\omega_{0}$ and amplitude $E_{0}$. It was argued that this approximation is valid in a regime, where the dielectric function of the bulk system can be replaced by unity, which is possible if $q_{0} \ll \sqrt{\omega_{0} / D}$ in $d=1$, and $q_{0} \ll \omega_{0} /\left(2 \pi e^{2} \nu_{2} D\right)$ in $d=2$. The semiclassical diffusive approximation which was made for the electrons dynamics is of course only valid for sufficiently small wave-vectors and frequencies. In particular, this sets an upper limit for the microwave frequency, namely $\omega_{0} \ll 1 / \tau_{e l}$. If these restrictions are satisfied, the dephasing rate due to microwaves is (for simplicity $\Gamma_{0}$ is set equal to zero and thus 


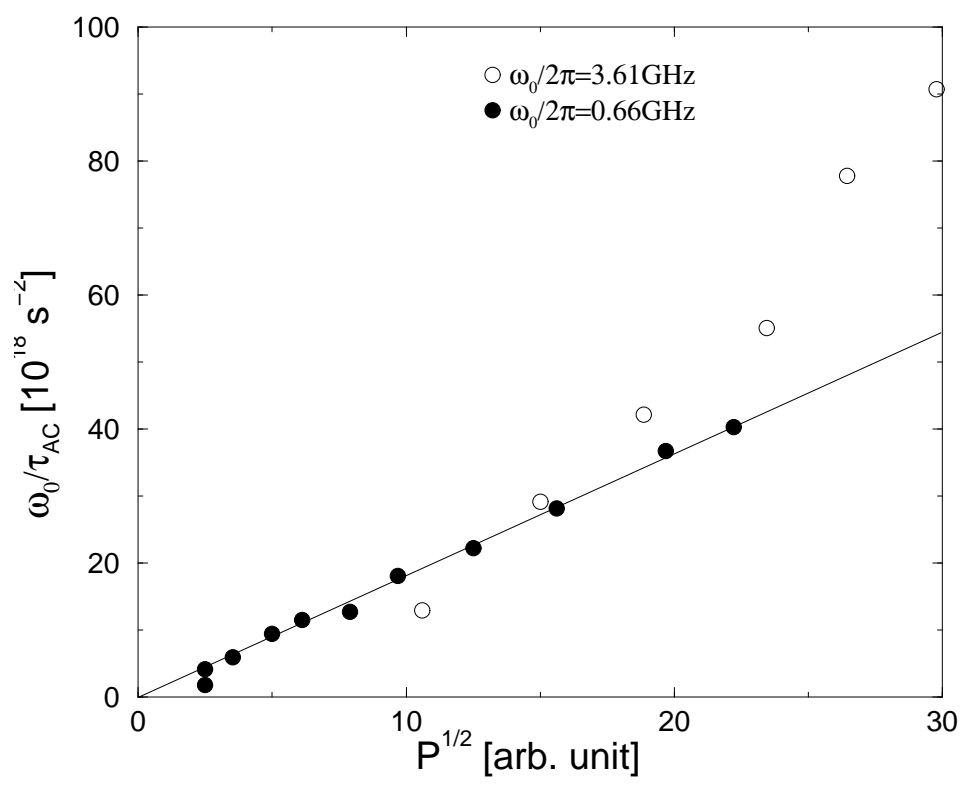

Figure 3.4: Data for $\omega_{0} / \tau_{A C}$ as function of $P^{1 / 2}$ from Fig.3 of Ref.[7(a)]. Here P is the microwave power coupled into the system. The solid line is a fit of the data at $\omega_{0} / 2 \pi=0.66 \mathrm{GHz}$ to the prediction $\omega_{0} / \tau_{A C} \propto P^{1 / 2}$, which is valid for small $P$.

$\gamma=0)$

$$
\frac{1}{\tau_{A C}} \propto \begin{cases}\omega_{0}^{2 / 5} P^{1 / 5} & \text { for }\left|V_{0}\right| \gg \omega_{0} \text { and } D\left(e E_{0}\right)^{2} \gg \omega_{0}^{2}, \\ \omega_{0}^{-2} P & \text { for }\left|V_{0}\right| \gg \omega_{0} \text { and } D\left(e E_{0}\right)^{2} \ll \omega_{0}^{2}, \\ \left(q_{0} \omega_{0}\right)^{1 / 3} P^{1 / 6} & \text { for }\left|V_{0}\right| \ll \omega_{0} \text { and } D\left|e E_{0}\right| q_{0} \gg \omega_{0}^{2}, \\ q_{0} \omega_{0}^{-1} \sqrt{P} & \text { for }\left|V_{0}\right| \ll \omega_{0} \text { and } D\left|e E_{0}\right| q_{0} \ll \omega_{0}^{2}\end{cases}
$$

with $P \propto E_{0}^{2}$.

Some time ago Wang and Lindelof [42] have measured $1 / \tau_{A C}$ as a function of $P$ in magnesium films. Their data from Ref.[7(a)] are reproduced in Fig.3.4. At $\omega_{0} / 2 \pi=0.66 \mathrm{GHz}$ the prediction $\omega_{0} / \tau_{A C} \propto P^{1 / 2}$ is in good agreement with the experiment. Although the data at $\omega_{0} / 2 \pi=3.61 \mathrm{GHz}$ cannot be fit by a straight line through the origin, the data roughly scale as $1 / \tau_{A C} \propto \omega_{0}^{-1}$ for fixed and small $P$. One should keep in mind, however, that in the experiments [42] the precise value of the microwave power coupled into the system was not measured, and the power axis for the two sets of data was rescaled differently. Further evidence for the $P^{1 / 2}$-law can be found in Fig.19 of Ref.[7(b)].

Recent measurements of $1 / \tau_{A C}$ by Webb et al. [46] suggest a $P^{1 / 5}$-law for large $P$ in a limited range of frequencies. The calculation presented here show that the $P^{1 / 5}$ law should hold as long as $\alpha \gg \max \{\beta, 1\}$, while for $\beta \gg \max \{\alpha, 1\}$ the prediction 
$1 / \tau_{A C} \propto \beta^{1 / 3} \propto P^{1 / 6}$ is made. Keeping in mind that $\alpha / \beta \propto \omega_{0}^{-1}$ a crossover from a $P^{1 / 5}$ - via a $P^{1 / 6}$ - to a $P^{1 / 2}$-behavior is predicted as the frequency is increased. The data shown in Fig.6 of Ref. [46] are consistent with the existence of such a crossover.

\subsection{The Diffuson in the presence of Nyquist noise}

In the previous chapters the Eikonal-expansion has been developed to study the Cooperon in an external field. The same formalism can be applied to the Diffuson. The Bethe-Salpeter equation for the Diffuson is obtained from Fig.2.4 by reversing the direction of the lower electron line. The calculations thus are the same as for the Cooperon, except the frequencies $\omega_{2}$ and $\omega_{2}^{\prime}$ are interchanged. This simply leads to interchanging the times $t$ and $t_{0}$ in the partial-differential equation in space-time coordinates and the result for the Diffuson, rescaled in the same way as the Cooperon in Eq.(2.57), is

$$
\begin{gathered}
\left(D \hat{\mathbf{P}}_{\mathbf{r}}^{2}+\partial_{t}\right) \mathcal{D}\left(\mathbf{r}, \mathbf{r}^{\prime} ; t, t^{\prime}, t_{0}\right)=\delta\left(\mathbf{r}-\mathbf{r}^{\prime}\right) \delta\left(t-t^{\prime}\right) \\
-i\left[V\left(\mathbf{r}, t+t_{0}\right)-V\left(\mathbf{r}, t-t_{0}\right)\right] \mathcal{D}\left(\mathbf{r}, \mathbf{r}^{\prime} ; t, t^{\prime}, t_{0}\right)
\end{gathered}
$$

The cutoff $\Gamma_{0}$ does not enter the equation for the Diffuson, since the non-interacting Diffuson $\mathrm{D}_{0}$ must have a diffusive pole (see Eq.(2.76) and the discussion preceding it). The diffusive nature of the electron dynamics should not be changed by interactions and thus no "dephasing" cutoff should be induced by the potential $V$. To see that Eq.(3.134) satisfies this condition, consider the linear change in the particle density $\delta n$ due to a scalar potential $\mathrm{V}_{d r i}$. In Sec.2.3 the correction to the current due to a vector potential $\mathbf{A}_{d r i}$ was studied. The leading semiclassical contribution was obtained from evaluating the Cooperon diagram in Fig.2.5. As already mentioned, Diffusons do not contribute to the spatially averaged $(\mathbf{Q}=\mathbf{0})$ current-current correlation function for symmetry reasons (in the model of disorder considered here). For the density-density correlation function this is not the case and the classical diffusive contribution is shown in Fig.3.5. Formally the diagrams are obtained from Fig.2.5 by replacing the current vertices by density vertices and the Cooperon by a Diffuson. The important difference arises in the frequency dependence of the Diffuson compared to the Cooperon. As noted above, the differential equation for the Diffuson is obtained from that for the Cooperon by interchanging the frequencies $\omega_{2}$ and $\omega_{2}^{\prime}$ in Fig.2.4. Comparing with Eqs.(2.46)-(2.48) one sees, that the Diffuson depends on the differences between the incoming and outgoing frequencies

$$
\begin{aligned}
\varepsilon & =\omega_{1}-\omega_{2}^{\prime}, \\
\varepsilon^{\prime} & =\omega_{1}^{\prime}-\omega_{2},
\end{aligned}
$$



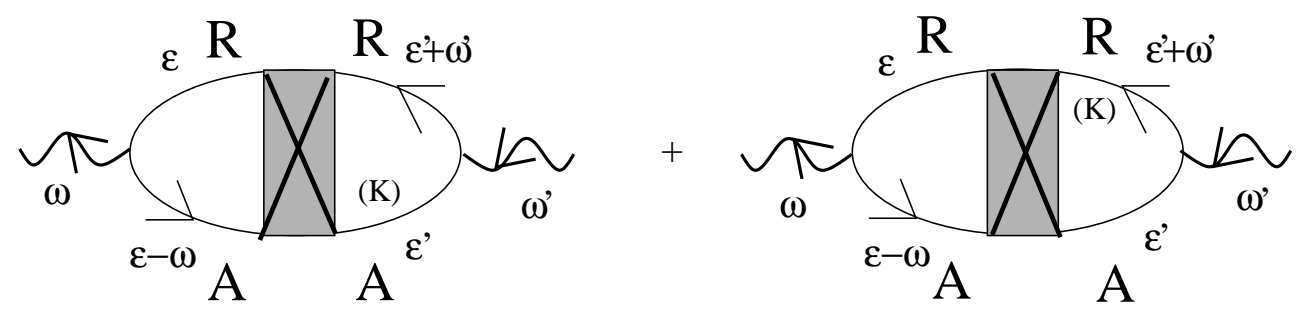

Figure 3.5: Diffuson correction to the density-density correlation function. The crossed shaded box is the Diffuson in the presence of the external field $V$.

and the difference of the frequency transfered to the external field in the upper and lower electron line

$$
\omega=\omega_{1}-\omega_{1}^{\prime}-\left(\omega_{2}-\omega_{2}^{\prime}\right)
$$

The diagrams in Fig. 3.5 thus give a contribution (for brevity all momentum dependence is suppressed, since it is of no importance for the following considerations)

$$
\delta n(\omega) \sim \int_{-\infty}^{\infty} d \varepsilon d \varepsilon^{\prime} d \omega^{\prime}\left[h\left(\varepsilon^{\prime}+\omega^{\prime}\right)-h\left(\varepsilon^{\prime}\right)\right] \mathcal{D}\left(\omega, \omega^{\prime}, 2 \varepsilon-2 \varepsilon^{\prime}-\omega-\omega^{\prime}\right) V_{d r i}\left(\omega^{\prime}\right),
$$

where $h(\varepsilon)=\tanh (\varepsilon \beta / 2)$. The frequency dependence of the averaged Green's functions is again neglected because only frequencies much smaller than $1 / \tau_{e l}$ are of interest. It is instructive to compare this expression with the weak localization correction to the current given in Eq.(2.62). In contrast to the Cooperon diagram, the integration variable $\varepsilon$ in Eq.(3.138) appears only at one position, namely the third argument of the Diffuson. Thus by changing this integration variable, Eq.(3.138) can be written as

$$
\delta n(\omega) \sim \frac{1}{2} \int_{-\infty}^{\infty} d \varepsilon d \varepsilon^{\prime} d \omega^{\prime}\left[h\left(\varepsilon^{\prime}+\omega^{\prime}\right)-h\left(\varepsilon^{\prime}\right)\right] \mathcal{D}\left(\omega, \omega^{\prime}, \varepsilon\right) V_{d r i}\left(\omega^{\prime}\right) .
$$

Now

$$
\int_{-\infty}^{\infty} \frac{d \varepsilon}{2 \pi} \mathcal{D}\left(\omega, \omega^{\prime}, \varepsilon\right)=\mathcal{D}\left(\omega, \omega^{\prime}, t_{0}=0\right)
$$

is just the Fourier transformation back to the time variable $t_{0}$ at point $t_{0}=0$. From the differential equation (3.134) it follows immediately, that for $t_{0}=0$ both contributions from the potential $V\left(t \pm t_{0}\right)$ cancel and thus the Diffuson is unaffected by the interaction. Since fluctuating external fields are related to electron-electron interactions by the fluctuation-dissipation theorem, such interactions can not lead to any cutoff in the diffusion propagator. Recall however, that Eq.(3.134) only describes 
"dephasing" effects due to weak fields with small frequency transfer that leave the classical motion of the electrons unaffected. In general electron-electron interactions will lead to a renormalization of the diffusion constant $D$ but will not regulize the diffusive pole. The Diffuson appearing in the density-density correlation function is unaffected by dephasing fields, since the two terms containing the field exactly cancel. After averaging over the field by means of the fluctuation-dissipation theorem this means, that the sum of all diagrams containing the two-particle interaction vanishes. If a class of diagrams is left out, a finite interaction correction remains. This is the case for the so called UCF-Diffuson appearing in the theory of universal conductance fluctuations [4,30]. There vertex corrections are left out, since the disorder average is performed over non self-averaging, physically independent systems and not over a macroscopic sample using an ergodicity hypothesis. But even if all classes of diagrams are kept, the cancellation of the field terms only occurs for the special frequency dependence of the Diffuson in the polarization bubble. If Diffusons appear with a different frequency dependence, they are indeed influenced by dephasing effects [33, 24, 47]. The diffusion pole is then cut off by a dephasing rate $1 / \tau_{D}\left(t_{0}\right)$ which now explicitly depends on $t_{0}$. In the following $1 / \tau_{D}\left(t_{0}\right)$ will be derived by means of the Eikonal method. The solution of Eq.(3.134) for the Diffuson is formally identical to that for the Cooperon derived in Sec.3.2 if the potential $V_{t_{0}}$ defined in Eq.(3.3) is replaced by

$$
V_{t_{0}}(\mathbf{r}, t)=i\left[V\left(\mathbf{r}, t+t_{0}\right)-V\left(\mathbf{r}, t-t_{0}\right)\right] .
$$

Making this replacement, one can use the result obtained in Sec.3.2 for arbitrary external fields. The important difference arises in the Nyquist noise correlator for the Fourier-transformed field $V_{t_{0}}(\mathbf{q}, \omega)=2 \sin \left(\omega t_{0}\right) V(\mathbf{q}, \omega)$. For the Diffuson this correlator is not independent of the time $t_{0}$ and is given by

$$
\left\langle V_{t_{0}}(\mathbf{q}, \omega) V_{t_{0}}\left(\mathbf{q}^{\prime}, \omega^{\prime}\right)\right\rangle_{n n}=-2(2 \pi)^{d+1} g(\mathbf{q}, \omega)\left[1-\cos \left(2 \omega t_{0}\right)\right] \delta\left(\omega+\omega^{\prime}\right) \delta\left(\mathbf{q}+\mathbf{q}^{\prime}\right) .
$$

Comparing this with Eq.(3.29) one notes, that there is no term $\sim \delta\left(\omega-\omega^{\prime}\right)$ and vertex corrections are now described by the term $\sim \cos \left(2 \omega t_{0}\right)$. The self-energy terms in both equations are still identical. The correlator $g(\mathbf{q}, \omega)$ is, as before, $g(\mathbf{q}, \omega)=-f_{q} \operatorname{coth}\left(\frac{\omega}{2 T}\right) \operatorname{Im} \epsilon^{-1}(\mathbf{q}, \omega)$. For the Cooperon the $\omega$-dependence of the dielectric function has been neglected since no divergences occured for small $\mathbf{q}$ and $\omega$. Here one has to take it into account, to ensure convergence of the integrals. Including the frequency dependence leads to different expressions for the correlator $g(\mathbf{q}, \omega)$, depending on the dimension $d$ (see Eqs.(2.79) - (2.81)). The calculations will be restricted to $d=2$ where

$$
g(\mathbf{q}, \omega)=\operatorname{coth}\left(\frac{\omega}{2 T}\right) \frac{2 \pi e^{2} \omega D \kappa_{2}}{\left(D \kappa_{2} \mathbf{q}\right)^{2}+\omega^{2}}
$$

and $\kappa_{2}=2 \pi e^{2} \nu_{2}$ is the inverse screening-length in two dimensions. Again one has to keep in mind, that due to the classical description of the external field all frequencies have to be restricted to $\omega<\omega_{c}$. 


\subsubsection{Infinite systems}

Using the results and notations of Sec. 3.2 one obtains

$$
\begin{aligned}
\left\langle\mathcal{D}\left(\mathbf{r}, \mathbf{r}^{\prime} ; t, t^{\prime}, t_{0}\right)\right\rangle_{n n} & =\Theta\left(t-t^{\prime}\right) \int \frac{d \mathbf{k}}{(2 \pi)^{d}} e^{i \mathbf{k} \cdot\left(\mathbf{r}-\mathbf{r}^{\prime}\right)} e^{-D \mathbf{k}^{2}\left(t-t^{\prime}\right)}\left\langle e^{-F\left(\mathbf{k}, t-t^{\prime} ; \mathbf{r}, t ; t_{0}\right)}\right\rangle_{n n} \\
& =: \Theta\left(t-t^{\prime}\right) \int \frac{d \mathbf{k}}{(2 \pi)^{d}} e^{i \mathbf{k} \cdot\left(\mathbf{r}-\mathbf{r}^{\prime}\right)} e^{-D \mathbf{k}^{2}\left(t-t^{\prime}\right)} e^{-\Gamma\left(\mathbf{k}, t, t^{\prime}, t_{0}\right)}
\end{aligned}
$$

The function $\Gamma\left(\mathbf{k}, t, t^{\prime}, t_{0}\right)$ now of course also depends on $t_{0}$ and up to second order in the external potential one gets $\Gamma\left(\mathbf{k}, t, t^{\prime}, t_{0}\right)=\Gamma_{1}\left(\mathbf{k}, t, t^{\prime}, t_{0}\right)+\Gamma_{2}\left(\mathbf{k}, t, t^{\prime}, t_{0}\right)$ with

$$
\begin{gathered}
\Gamma_{1}\left(\mathbf{k}, t, t^{\prime}, t_{0}\right)=\int \frac{d \mathbf{q} d \omega}{(2 \pi)^{d+1}} g(\mathbf{q}, \omega)\left[1-\cos \left(2 \omega t_{0}\right)\right] \frac{1-e^{-\left(E_{\mathbf{k}}(\mathbf{q})-i \omega\right)\left(t-t^{\prime}\right)}}{E_{\mathbf{k}}(\mathbf{q})-i \omega} \\
\times \frac{1-e^{-\left(E_{\mathbf{k}}(-\mathbf{q})+i \omega\right)\left(t-t^{\prime}\right)}}{E_{\mathbf{k}}(-\mathbf{q})+i \omega}, \\
\Gamma_{2}\left(\mathbf{k}, t, t^{\prime}, t_{0}\right)=\int \frac{d \mathbf{q} d \omega}{(2 \pi)^{d+1}} g(\mathbf{q}, \omega)\left[1-\cos \left(2 \omega t_{0}\right)\right] \frac{2 D \mathbf{q}^{2}}{\left(E_{\mathbf{k}}(\mathbf{q})-i \omega\right)\left(E_{\mathbf{k}}(-\mathbf{q})+i \omega\right)} \\
\times\left[\left(t-t^{\prime}\right)-\frac{1-e^{-\left(E_{\mathbf{k}}(-\mathbf{q})+i \omega\right)\left(t-t^{\prime}\right)}}{E_{\mathbf{k}}(-\mathbf{q})+i \omega}-\frac{1-e^{-\left(E_{\mathbf{k}}(\mathbf{q})-i \omega\right)\left(t-t^{\prime}\right)}}{E_{\mathbf{k}}(\mathbf{q})-i \omega}+\frac{1-e^{-2 D \mathbf{q}^{2}\left(t-t^{\prime}\right)}}{2 D \mathbf{q}^{2}}\right] .
\end{gathered}
$$

Obviously the averaged Diffuson $\left\langle\mathcal{D}\left(\mathbf{r}, \mathbf{r}^{\prime} ; t, t^{\prime}, t_{0}\right)\right\rangle_{n n}$ depends only on $t_{0}$ and the time and position differences $t-t^{\prime} ; \mathbf{r}-\mathbf{r}^{\prime}$. Therefore the definitions $\tilde{D}\left(\mathbf{r}-\mathbf{r}^{\prime}, t-t^{\prime}, t_{0}\right):=$ $\left\langle\mathcal{D}\left(\mathbf{r}, \mathbf{r}^{\prime} ; t, t^{\prime}, t_{0}\right)\right\rangle_{n n}$ and $\Gamma\left(\mathbf{k}, t-t^{\prime}, t_{0}\right):=\Gamma\left(\mathbf{k}, t, t^{\prime}, t_{0}\right)$ are introduced. According to Eq.(3.144) $\tilde{D}\left(\mathbf{r}, t, t_{0}\right)$ is represented as

$$
\tilde{D}\left(\mathbf{r}, t, t_{0}\right)=\Theta(t) \int \frac{d \mathbf{k}}{(2 \pi)^{d}} e^{i \mathbf{k} \cdot \mathbf{r}} e^{-D \mathbf{k}^{2} t} e^{-\Gamma\left(\mathbf{k}, t, t_{0}\right)} .
$$

The time-scale $\tau_{D}$ that cuts of the divergence of $\tilde{D}\left(\mathbf{r}, t, t_{0}\right)$ for large times $t$ is determined by the $\mathbf{k}=\mathbf{0}$ mode of $\Gamma\left(\mathbf{k}, t, t_{0}\right)$ and one defines, analogous to the dephasing time for the Cooperon: $\Gamma\left(\mathbf{k}=\mathbf{0}, \tau_{D}, t_{0}\right)=1$. In contrast to the Cooperon case, no physical observable quantity like the weak localization correction is looked at to define $\tau_{D}$. Therefore the frequency $\varepsilon$ does not enter here, but $\tau_{D}$ also depends on $t_{0}$, i.e. the decay rate of the Diffuson is not simply characterized by a single time scale, but rater by $\tau_{D}$ and $t_{0}[24,47]$. With the notation $\Gamma\left(t, t_{0}\right):=\Gamma\left(\mathbf{k}=\mathbf{0}, t, t_{0}\right)$ one gets

$$
\begin{aligned}
\Gamma\left(t, t_{0}\right)= & 2 \operatorname{Re} \int \frac{d \mathbf{q} d \omega}{(2 \pi)^{d+1}} g(\mathbf{q}, \omega)\left[1-\cos \left(2 \omega t_{0}\right)\right] \\
& \times\left[\frac{D \mathbf{q}^{2} t}{\left(D \mathbf{q}^{2}\right)^{2}+\omega^{2}}+\frac{e^{-\left(D \mathbf{q}^{2}-i \omega\right) t}-1}{\left(D \mathbf{q}^{2}-i \omega\right)^{2}}\right] .
\end{aligned}
$$


Comparing this expression with the corresponding result for the Cooperon, Eq.(3.44), one finds that the terms in braces are just the first two terms in Eq.(3.44). Recall that this is nothing but the contribution from the self-energy corrections. Obviously both equations are the same if the vertex parts, i.e. $\cos \left(2 \omega t_{0}\right)$ in Eq.(3.148) and the third term in braces in Eq.(3.44) are dropped. This statement is already clear from comparing the two correlators in Eq.(3.29) and Eq.(3.142). Thus the interaction corrections to the Diffuson are different from that for the Cooperon only due to vertex corrections. Now $\Gamma\left(t, t_{0}\right)$ will be calculated in $d=2$ with $\omega_{c}=T$. Using Eq.(3.143) and substituting $x:=D \mathbf{q}^{2} t$ and $y:=\omega t$ one arrives at

$$
\begin{aligned}
\Gamma\left(t, t_{0}\right)=\frac{T e^{2} \kappa_{2} t^{2}}{\pi} \operatorname{Re} \int_{0}^{\infty} d x \int_{-T t}^{T t} d y \frac{1-\cos \left(2 y t_{0} / t\right)}{D \kappa_{2}^{2} t x+y^{2}} \\
\times\left[\frac{e^{-(x-i y)}-1}{(x-i y)^{2}}+\frac{x}{x^{2}+y^{2}}\right] .
\end{aligned}
$$

The frequency-dependence of $g(\mathbf{q}, \omega)$ is needed to cut off the divergence for $\mathbf{q} \rightarrow 0$ (i.e. $x \rightarrow 0$ ). To isolate this singular contribution, Eq.(3.149) is rewritten as

$$
\begin{aligned}
\Gamma\left(t, t_{0}\right)= & 2 \frac{T e^{2} \kappa_{2} t^{2}}{\pi} \operatorname{Re} \int_{0}^{\infty} d x \int_{0}^{T t} d y \frac{1-\cos \left(2 y t_{0} / t\right)}{D \kappa_{2}^{2} t x+y^{2}} \\
& \times\left[\frac{e^{-(x-i y)}-e^{i y}}{(x-i y)^{2}}+\frac{e^{i y}-1}{(x-i y)^{2}}+\frac{x}{x^{2}+y^{2}}\right] .
\end{aligned}
$$

Only in the second term in braces the frequency-dependence of $g(\mathbf{q}, \omega)$ is needed to ensure convergence for $x \rightarrow 0$. Thus it may be neglected in the other terms and $1 /\left(D \kappa_{2}^{2} t x+y^{2}\right)$ is replaced by $1 /\left(D \kappa_{2}^{2} t x\right)$ to evaluate their contributions. Introducing the abbreviation $b:=D \kappa_{2}^{2} t$, this leads to the following integrals:

$$
\begin{array}{r}
I_{1}:=\int_{0}^{\infty} d x \int_{-T t}^{T t} d y \frac{1-\cos \left(2 y t_{0} / t\right)}{b x+y^{2}} \frac{e^{-(x-i y)}-e^{i y}}{(x-i y)^{2}} \\
\approx \int_{0}^{\infty} d x \int_{-\infty}^{\infty} d y \frac{1-\cos \left(2 y t_{0} / t\right)}{b x} \frac{e^{-(x-i y)}-e^{i y}}{(x-i y)^{2}} \\
=\frac{2 \pi}{b}\left(\frac{2 t_{0}}{t}-1\right) \Theta\left(\frac{2 t_{0}}{t}-1\right) \int_{0}^{\infty} d x e^{-x\left(2 t_{0} / t-1\right)} \frac{e^{-x}-1}{x} \\
I_{2}:=\int_{0}^{\infty} d x \int_{0}^{T t} d y \frac{1-\cos \left(2 y t_{0} / t\right)}{b x+y^{2}} \frac{x}{x^{2}+y^{2}} \\
\approx \int_{0}^{\infty} d x \int_{-T t}^{T t} d y \frac{1-\cos \left(2 y t_{0} / t\right)}{b} \frac{1}{x^{2}+y^{2}}=\int_{0}^{T t} d y \frac{1-\cos \left(2 y t_{0} / t\right)}{b} \frac{\pi}{2 y},
\end{array}
$$


and

$$
\begin{aligned}
I_{3} & :=\int_{0}^{\infty} d x \int_{0}^{T t} d y \frac{1-\cos \left(2 y t_{0} / t\right)}{b x+y^{2}} \frac{e^{i y}-1}{(x-i y)^{2}} \\
& =\int_{0}^{T t} d y\left(1-\cos \left(2 y t_{0} / t\right)\right)\left(e^{i y}-1\right)\left[-\frac{i b-\frac{\pi}{2} b+y-i b \ln \left(\frac{b}{y}\right)}{i y^{2}(i b+y)^{2}}\right] \\
& \approx \int_{0}^{T t} d y\left(1-\cos \left(2 y t_{0} / t\right)\right)\left(e^{i y}-1\right) \frac{y-\frac{\pi}{2} b-i b \ln \left(\frac{b}{y}\right)}{i y^{2} b^{2}},
\end{aligned}
$$

where $D \kappa_{2}^{2} \gg T$ was used to obtain the last line. Inserting these results into Eq. (3.150) yields

$$
\begin{array}{r}
\Gamma\left(t, t_{0}\right)=\frac{T e^{2} \kappa_{2} t^{2}}{\pi} \operatorname{Re}\left\{2 \int _ { 0 } ^ { T t } d y ( 1 - \operatorname { c o s } ( 2 y t _ { 0 } / t ) ) \left[\frac{\pi}{2 b y}\right.\right. \\
\left.\left.+\frac{e^{i y}-1}{i y^{2} b^{2}}\left(y-\frac{\pi}{2} b-i b \ln \left(\frac{b}{y}\right)\right)\right]+I_{1}\right\} \\
=\frac{T e^{2} \kappa_{2} t^{2}}{\pi}\left\{2 \int _ { 0 } ^ { T t } d y ( 1 - \operatorname { c o s } ( 2 y t _ { 0 } / t ) ) \left[\frac{\pi}{2 b y}\left(1-\frac{\sin (y)}{y}\right)+\frac{\sin (y)}{b^{2} y}\right.\right. \\
\left.\left.-\frac{\cos (y)-1}{b y^{2}} \ln \left(\frac{b}{y}\right)\right]+I_{1}\right\} .
\end{array}
$$

For $D \kappa_{2}^{2} \gg T$ one finally gets

$$
\begin{array}{r}
\Gamma\left(t, t_{0}\right)=\frac{2 T e^{2} \kappa_{2} t^{2}}{\pi}\left\{\int _ { 0 } ^ { T t } d y ( 1 - \operatorname { c o s } ( 2 y t _ { 0 } / t ) ) \frac { \pi } { 2 b } \left[\frac{1}{y}\left(1-\frac{\sin (y)}{y}\right)\right.\right. \\
\left.\left.-\frac{2}{\pi} \frac{\cos (y)-1}{y^{2}} \ln \left(\frac{b}{y}\right)\right]+\frac{I_{1}}{2}\right\} .
\end{array}
$$

$\Gamma\left(t, t_{0}\right)$ can be evaluated in the three limiting cases $t_{0} \gg t, 1 / T \ll t_{0} \ll t$ and $t_{0} \ll 1 / T$. For $t_{0} \gg t$ the term $I_{1}$ is of order unity and can be neglected. Since it is zero in the other cases, $I_{1}$ does not contribute. For $t_{0} \gg t$ the strongly oscillating vertex-corrections term $\cos \left(2 y t_{0} / t\right)$ gives a negligible contribution and thus

$$
\begin{gathered}
\Gamma\left(t, t_{0}\right)=\frac{T t}{2 \pi D \nu_{2}} \int_{0}^{T t} d y\left[\left(\frac{1}{y}-\frac{\sin (y)}{y^{2}}\right)-\frac{2}{\pi} \frac{\cos (y)-1}{y^{2}} \ln \left(\frac{b}{y}\right)\right] \\
\quad \approx \frac{T t}{2 \pi D \nu_{2}}\left[\int_{0}^{T t} d y \frac{1-\cos (y)}{y}-\frac{2}{\pi} \int_{0}^{\infty} d y \frac{\cos (y)-1}{y^{2}} \ln \left(\frac{b}{y}\right)\right] .
\end{gathered}
$$

Integrating the last term by parts and neglecting terms of order unity yields

$$
\begin{aligned}
& \Gamma\left(t, t_{0}\right)=\frac{T t}{2 \pi D \nu_{2}}\left[\int_{0}^{T t} d y \frac{1-\cos (y)}{y}+\frac{2}{\pi} \ln (b) \int_{0}^{\infty} d y \frac{\sin (y)}{y}\right] \\
\approx & \frac{T t}{2 \pi D \nu_{2}}\left[\int_{1}^{T t} d y \frac{1}{y}+\ln (b)\right]=\frac{T t}{2 \pi D \nu_{2}} \ln \left(T D \kappa_{2}^{2} t^{2}\right) \text { for } t_{0} \gg t .
\end{aligned}
$$


In the case $1 / T \ll t_{0} \ll t$ dominant contributions come from the region $y>1$ and one can neglect the terms with $\sin (y) / y$ and $\cos (y) / y^{2}$ :

$$
\Gamma\left(t, t_{0}\right)=\frac{T t}{2 \pi D \nu_{2}} \int_{0}^{T t} d y\left[1-\cos \left(2 y t_{0} / t\right)\right]\left[\frac{1}{y}+\frac{2}{\pi y^{2}} \ln \left(\frac{b}{y}\right)\right] .
$$

Substituting $z:=2 y t_{0} / t$ the second term becomes of the form as the one in Eq.(3.156) and is evaluated in the same way, resulting in

$$
\begin{aligned}
& \Gamma\left(t, t_{0}\right)=\frac{T t}{2 \pi D \nu_{2}}\left[\int_{0}^{T t} d y \frac{1-\cos \left(2 y t_{0} / t\right)}{y}+\frac{2}{\pi} \frac{2 t_{0}}{t} \ln \left(b \frac{2 t_{0}}{t}\right) \int_{0}^{\infty} d z \frac{\sin (z)}{z}\right] \\
& \approx \frac{T t}{2 \pi D \nu_{2}}\left[\ln \left(2 T t_{0}\right)+\frac{2 t_{0}}{t} \ln \left(2 D \kappa_{2}^{2} t_{0}\right)\right] \approx \frac{T t}{2 \pi D \nu_{2}} \ln \left(2 T t_{0}\right) \quad \text { for } 1 / T \ll t_{0} \ll t .
\end{aligned}
$$

Finally for $t_{0} \ll 1 / T$ the function $\cos \left(2 y t_{0} / t\right)$ is expanded to first order, leading to

$$
\begin{aligned}
\Gamma\left(t, t_{0}\right) & =\frac{T t}{2 \pi D \nu_{2}} \int_{0}^{T t} d y 2 y^{2}\left(\frac{t_{0}}{t}\right)^{2}\left(\frac{1}{y}-\frac{\sin (y)}{y^{2}}-\frac{2}{\pi} \frac{\cos (y)-1}{y^{2}} \ln \left(\frac{b}{y}\right)\right) \\
& \approx \frac{T^{3} t_{0}^{2} t}{2 \pi D \nu_{2}} \quad \text { for } t_{0} \ll 1 / T .
\end{aligned}
$$

Now the cutoff $1 / \tau_{D}$ for the Diffuson is determined by the condition $\Gamma\left(\tau_{D}, t_{0}\right)=1$. This yields

$$
\frac{1}{\tau_{D}}=\frac{T}{2 \pi D \nu_{2}} \ln \left(T D \kappa_{2}^{2} \tau_{D}^{2}\right) \quad \text { for } t_{0} \gg t
$$

and to leading order

$$
\frac{1}{\tau_{D}} \approx \frac{T}{2 \pi D \nu_{2}} \ln \left[\frac{D \kappa_{2}^{2}}{T}\left(2 \pi D \nu_{2}\right)^{2}\right] \quad \text { for } t_{0} \gg t
$$

Correspondingly

$$
\begin{gathered}
\frac{1}{\tau_{D}}=\frac{T}{2 \pi D \nu_{2}} \ln \left(2 T t_{0}\right) \quad \text { for } 1 / T \ll t_{0} \ll t, \\
\frac{1}{\tau_{D}}=\frac{T^{3} t_{0}^{2}}{2 \pi D \nu_{2}} \quad \text { for } t_{0} \ll 1 / T .
\end{gathered}
$$

The above results have previously been derived within a path-integral approach [24, 33]. Obviously the cutoff $1 / \tau_{D}$ vanishes for $t_{0} \rightarrow 0$. As explained in the introduction to this section, this just demonstrates the fact that the diffusive pole in the polarization function is unaffected by interactions, which is a consequence of 
particle-number conservation [13]. On the other hand, for $t_{0} \rightarrow \infty$ vertex corrections become unimportant and one obtains the UCF-Diffuson that appears in the theory of universal conductance fluctuations [30]. Even though vertex-corrections become unimportant for large times $t_{0}$, the vertex-term $\sim \cos \left(2 y t_{0} / t\right)$ is needed to ensure that the two-dimensional integral in Eq.(3.150) exists and is independent of the order of integration. If only the linear in time contribution in Eq.(3.148) is kept and vertex-corrections are ignored, i.e.

$$
\Gamma\left(t, t_{0}\right) \rightarrow \tilde{\Gamma}(t)=2 \operatorname{Re} \int \frac{d \mathbf{q} d \omega}{(2 \pi)^{d+1}} g(\mathbf{q}, \omega) \frac{D \mathbf{q}^{2} t}{\left(D \mathbf{q}^{2}\right)^{2}+\omega^{2}}
$$

and the corresponding cutoff $1 / \tilde{\tau}_{D}$ is determined by $\tilde{\Gamma}\left(\tilde{\tau}_{D}\right)=1$ one obtains

$$
\frac{1}{\tilde{\tau}_{D}}=2 \operatorname{Re} \int \frac{d \mathbf{q} d \omega}{(2 \pi)^{d+1}} g(\mathbf{q}, \omega) \frac{D \mathbf{q}^{2}}{\left(D \mathbf{q}^{2}\right)^{2}+\omega^{2}} .
$$

This is just the equation obtained in Refs. [29, 30] within a diagrammatic perturbation theory. Since the integral diverges at $\omega=0$ the cutoff $1 / \tilde{\tau}_{D}$ is substituted self-consistently into the diffusion propagator in the integrand, $D \mathbf{q}^{2} \rightarrow D \mathbf{q}^{2}+1 / \tilde{\tau}_{D}$. This procedure produces exactly the same result as the Eikonal expansion for $t_{0} \gg t$, which is given in Eq.(3.162).

\subsubsection{Finite systems}

For mesoscopic systems the momentum integral in Eq.(3.148) is replaced by a sum and the $\mathbf{q}=\mathbf{0}$ mode is excluded (see the discussion regarding Eq.(3.46)). At $\tau_{D} E_{c} \gg 1$ the exponentially damped term can again be neglected and consequently Eq.(3.148) becomes

$\Gamma\left(t, t_{0}\right)=2 \operatorname{Re} \frac{1}{L^{d}} \sum_{\mathbf{q} \neq \mathbf{0}} \int_{-\infty}^{\infty} \frac{d \omega}{2 \pi} g(\mathbf{q}, \omega)\left[1-\cos \left(2 \omega t_{0}\right)\right]\left[\frac{D \mathbf{q}^{2} t}{\left(D \mathbf{q}^{2}\right)^{2}+\omega^{2}}-\frac{1}{\left(D \mathbf{q}^{2}-i \omega\right)^{2}}\right]$.

Now, since the $\mathbf{q}=\mathbf{0}$ mode does not contribute, one faces no convergence problems for small momenta and therefore the frequency dependence in the denominator of $g(\mathbf{q}, \omega)$ may be neglected, i.e. one can use the approximation given in Eq.(3.28), which was already used for the Cooperon.

In the limit $t_{0} \rightarrow \infty$ the vertex part $\sim \cos \left(2 \omega t_{0}\right)$ vanishes. It was shown in Sec. 3.3 .3 that in mesoscopic systems vertex corrections also do not contribute to $\tau_{n n}$ and thus in such systems $\tau_{D}\left(\right.$ at $\left.t_{0} \rightarrow \infty\right)$ coincides with the dephasing time (at $\varepsilon \rightarrow 0$ ):

$$
\frac{1}{\tau_{D}}=\frac{1}{\tau_{n n}} \quad \text { for } t_{0} \rightarrow \infty \text { and } \frac{1}{\tau_{D}} \ll E_{c} .
$$


In the opposite limit $t_{0} T \ll 1$ one can expand $\cos \left(2 \omega t_{0}\right)$ to first order and obtains

$$
\Gamma\left(t, t_{0}\right)=\frac{8 T}{\nu_{d}} \operatorname{Re} \frac{1}{L^{d}} \sum_{\mathbf{q} \neq \mathbf{0}} \int_{0}^{T} \frac{d \omega}{2 \pi} \frac{1}{D \mathbf{q}^{2}} 2 \omega^{2} t_{0}^{2}\left[\frac{D \mathbf{q}^{2} t}{\left(D \mathbf{q}^{2}\right)^{2}+\omega^{2}}-\frac{1}{\left(D \mathbf{q}^{2}-i \omega\right)^{2}}\right]
$$

Here again $\operatorname{coth}(\omega /(2 T))$ was approximated by $2 T / \omega$ for $\omega<T$. Under the conditions $\tau_{D} E_{c} \gg 1$ and $T \tau_{D} \gg 1$ one obtains to leading order

$$
\Gamma\left(t, t_{0}\right)=\frac{8 T t_{0}^{2} t}{\pi \nu_{d}} \frac{1}{L^{d}} \sum_{\mathbf{q} \neq \mathbf{0}}\left[T-D \mathbf{q}^{2} \arctan \left(\frac{T}{D \mathbf{q}^{2}}\right)\right] .
$$

The dominant contribution to this sum comes from the regime $D \mathbf{q}^{2}<T$. The sum is thus estimated by

$$
\Gamma\left(t, t_{0}\right)=\frac{8 T t_{0}^{2} t}{\pi \nu_{d}} \frac{1}{L^{d}} \sum_{\mathbf{q} \neq \mathbf{0}}^{|\mathbf{q}|<\sqrt{T / D}} T
$$

Since it is assumed that $T \gg E_{c}$ the volume in momentum-space that is summed over is large in comparison to $(2 \pi / L)^{d}$, i.e. $\sqrt{T / D} \gg 1 / L$. Thus the number of states with $|\mathbf{q}|<\sqrt{T / D}$ is approximated by the volume of the $d$-dimensional sphere with radius $\sqrt{T / D}$ divided by $(2 \pi / L)^{d}$. This yields

$$
\Gamma\left(t, t_{0}\right) \sim \frac{T^{2} t_{0}^{2} t}{\nu_{d}}\left[\frac{T}{E_{c}}\right]^{\frac{d}{2}}
$$

Using the definition $\Gamma\left(\tau_{D}, t_{0}\right)=1$ one finally arrives at

$$
\frac{1}{\tau_{D}} \sim \frac{T^{2} t_{0}^{2}}{\nu_{d}}\left[\frac{T}{E_{c}}\right]^{\frac{d}{2}} \quad \text { for } E_{c} \ll T \ll \frac{1}{t_{0}} .
$$




\section{Chapter 4}

\section{Non-linear response in mesoscopic metal rings}

\subsection{Introduction}

A common method to gain information about physical systems is to measure the response to an external perturbation, e.g. a time-dependent field. To compare the experimental results with theoretical predictions one thus needs to derive a response function that describes the change of the measured quantity with respect to the applied field. Such a function can be derived by expanding the observable in powers of the perturbation (see Appendix A). Explicit evaluation of higher order terms usually becomes quite cumbersome but if the external field is not too strong, it is often sufficient to study the linear response. In mesoscopic systems however, nonlinear effects become much more important. Even if an external field is too weak to change the classical motion of the electrons in the sample, it still affects their phase. Since in mesoscopic samples electrons can propagate coherently through the whole probe, such systems are extremely sensitive to phase changes. Nonlinear effects in mesoscopic Aharonov-Bohm rings were first studied by Kravtsov and collaborators in Refs. [56, 57]. The external perturbation is in this case a timedependent magnetic flux $\phi(t)$ that has a static component $\phi$ and a part that oscillates with frequency $\varepsilon$,

$$
\phi(t)=\phi+\phi_{\varepsilon} \sin (\varepsilon t)
$$

By Faraday's law of induction, the oscillating part generates a time-dependent electric field directed along the circumference of the ring, $E(t)=E_{\varepsilon} \cos (\varepsilon t)$, with amplitude

$$
e L E_{\varepsilon}=2 \pi \varepsilon \frac{\phi_{\varepsilon}}{\phi_{0}} .
$$


Here $L$ is the circumference of the ring and $\phi_{0}$ is the flux quantum. One would like to know the induced current around the ring. In the limit $\varepsilon \rightarrow 0$ this is just the usual persistent current [49,6]. The linear response to the time-dependent component of the flux has been studied by several authors [51, 52, 53, 54]. In Refs. [51, 52, 53] the kinetic equation for an electron gas that is coupled to an external reservoir via a relaxation rate $\gamma_{r e l}$ was examined. It was found, that the conductivity does depend critically on the statistical ensemble (canonical or grand-canonical) and on whether $\gamma_{r e l}$ is smaller or lager than the level-spacing $\Delta$.

For frequencies in the range between $10^{8}$ and $10^{13} \mathrm{~Hz}$, which for experimentally relevant rings [50,55] corresponds to $\Delta \ll \varepsilon \ll \tau_{\text {el }}^{-1}$ nonlinear effects become important as well. The ring geometry leads to strong interference effects in the electronic transport and thus such systems are sensitive to dephasing effects that are induced by the external field. In Sec.3.4 the dephasing due to microwave radiation was studied for bulk systems by solving the differential equation for the Cooperon in a time-dependent external field. For spatially homogeneous fields, an exact solution is possible. The situation investigated here is slightly different, since no additional microwave radiation is present, but the fluctuating dephasing field is the external driving field itself. As noticed by Kravtsov et al. [57, 56], the induced current is still governed by the Cooperon and described by an expression similar to Eq.(2.68). Since the current-Cooperon relation is already linear in the field, it is clear, that the current is nonlinear in $E_{\varepsilon}$, if the Cooperon depends on this field as well. Aronov and Kravtsov (AK) derived an 'exact' solution for this problem in Ref. [57]. In this context 'exact' means, that the partial differential equation for the Cooperon in the external field was solved exactly. However, the current-Cooperon relation is of course an approximation which is obtained from the standard disorder perturbation theory. AK realized different physical regimes, depending on the relative order of magnitude of the quantities $E_{\varepsilon}$, frequency $\varepsilon$ and intrinsic dephasing rate $1 / \tau_{\text {int }}$ (i.e. the dephasing that is not caused by the external field). Among others, there exists a 'perturbative' regime, where the Cooperon can be expanded to first order in the field, which is equivalent to calculating the current to second order in $E_{\varepsilon}$. According to Ref. [57] this expansion is possible as long as the field induced dephasing rate $1 / \tau_{A C} \sim\left(e^{2} E_{\omega}^{2} \varepsilon^{2} D\right)^{1 / 5}$ (compare Eq.(3.108)) is smaller than $\max \left[1 / \tau_{\text {int }}, \varepsilon /(2 \pi)\right]$. The quadratic response has first been calculated by Kravtsov and Yudson [56] (KY), who found that in second order the time-dependent field induces (among other terms that oscillate) a time-independent non-equilibrium current $I_{0}^{(2)}$. Calculating the disorder average of this current perturbatively, KY found that it has the peculiar property that for frequencies exceeding the Thouless energy $E_{c}$ the average of $I_{0}^{(2)}$ does not vanish exponentially, but only as $\varepsilon^{-2}$. This is in disagreement with the intuitive expectation that the external frequency $\varepsilon$ leads to a similar exponential suppression of this mesoscopic non-equilibrium current as a dephasing rate in the case of the equilibrium persistent current [58, 59]. The same current-Cooperon relation that was the starting point of the calculations by KY was 
used in Sec.3.3.3 to derive the dephasing rate due to Nyquist noise (electron-electron interactions) in the presence of an external driving field $\mathbf{E}_{d r i}$ with frequency $\varepsilon$. It was outlined that this procedure is only controlled for frequencies $\varepsilon>E_{c}$ since otherwise the dephasing rate becomes smaller than $\Delta$. The work by Kravtsov et al. was motivated by the close connection between non-linear response and electron-electron interactions (a point that will be discussed in more detail in Chap.(5)). Thus if a formalism fails to describe interaction effects for frequencies $\varepsilon<E_{c}$, one can not expect it to yield correct response functions in this frequency range. Indeed, it will be explicitly demonstrated below, that in the discrete spectrum limit the quadratic response is completely different from the prediction of the diagrammatic disorder averaging. In fact, in this chapter it will be shown that the term considered by KY is then not constant, but grows linearly in time, a result which can be understood simply in terms of Fermi's golden rule of time-dependent perturbation theory. All calculations will be performed for a non-interacting electron gas. Since there are no inelastic processes in this system, the energy spectrum is truely discrete. The use of such a simplified model is justified by the Fermi liquid theory which describes the excitation spectrum of an interacting system by quasiparticles with excitation energy $\xi_{\alpha}$ and lifetime $\gamma_{q p}^{-1}\left(\xi_{\alpha}\right)$. From this point of view the condition $\gamma_{q p}\left(\xi_{\alpha}\right)<\Delta$ rather than $1 / \tau_{\varphi}(\varepsilon)<\Delta$ should define the energy scale which limits the validity of the following considerations. For low enough temperatures $(T \rightarrow 0) \gamma_{q p}$ is dominated by inelastic electron-electron scattering [20], $\gamma_{q p} \approx 1 / \tau_{e e} \sim \nu_{d}^{-1}\left(\xi_{\alpha} / D\right)^{d / 2}$. Since the energy dependence is identical to that of $1 / \tau_{n n}$ (see Eq.(3.70)) the inelastic level broadening will be smaller than $\Delta$ for $\xi_{\alpha}<E_{c}$. It is well known, that the canonical thermodynamic equilibrium current for non-interacting electrons is governed by an energy interval of size $E_{c}$ around the Fermi energy [59,75]. It seems therefore reasonable to assume, that for frequencies $\varepsilon<E_{c}$ the response function should also be determined by this part of the spectrum. This assumption will be verified numerically in Sec.4.4. In conclusion, the results derived in this chapter should be valid at low temperatures for external frequencies $\varepsilon<E_{c}$. The discrete spectrum limit becomes of increasing experimental relevance, since due to the progress in nano-scale technology the size of the investigated rings is continuously decreased and therewith the Thouless energy of the systems is increased [48].

It is important to point out the difference between the current considered here and the direct current due to the usual photovoltaic effect. It is well known [60] that irradiation of a medium without an inversion center by an alternating electric field can give rise to a direct current (photovoltaic effect). The lack of inversion symmetry can be due to impurities and defects in a finite sample. For mesoscopic junctions the photovoltaic direct current has been studied in Ref. [61]. In this case the average current vanishes, because disorder averaging restores the inversion symmetry. In the case studied here, however, the direct current induced in a mesoscopic ring threaded by a magnetic flux is calculated. Because the magnetic flux breaks the time-reversal symmetry, the direct current considered here has a finite disorder average. Thus, 
the physical origin of a mesoscopic non-equilibrium current discussed in this work is quite different from Ref. [61].

\subsection{The quadratic response function: What is wrong with the Green's function approach?}

The system considered here consists of non-interacting disordered electrons of mass $m$ confined to a thin ring and threaded by the time-dependent magnetic flux given in Eq.4.1). In the absence of the oscillating flux (i.e. for $\phi_{\varepsilon}=0$ in Eq.(4.1)) the Hamiltonian is given by

$$
\hat{H}_{0}=\sum_{\mathbf{p}} \frac{\mathbf{p}^{2}(\varphi)}{2 m} \hat{\Psi}_{\mathbf{p}}^{\dagger} \hat{\Psi}_{\mathbf{p}}+\int d \mathbf{r} U(\mathbf{r}) \hat{\Psi}^{\dagger}(\mathbf{r}) \hat{\Psi}(\mathbf{r}),
$$

with $\mathbf{p}(\varphi)=\mathbf{p}+(2 \pi / L) \varphi \mathbf{e}_{x}$, where $\mathbf{e}_{x}$ is the unit vector in $x$-direction and $\varphi=\phi / \phi_{0}$. As usual, the coordinate along the circumference is called the $x$-direction, and the ring geometry is modeled by periodic boundary conditions in that direction. The corresponding component of the wave vector is thus quantized by $p_{x}=(2 \pi / L) n_{x}$ with integer numbers $n_{x}$. Suppose that $\hat{H}_{0}$ is diagonalized for the given realization of the disorder. The time-independent part of the Hamiltonian is then $\hat{H}_{0}=\sum_{\alpha} \varepsilon_{\alpha} \hat{c}_{\alpha}^{\dagger} \hat{c}_{\alpha}$, where $\varepsilon_{\alpha}$ are the exact electronic eigenenergies for fixed disorder, which are labeled by appropriate quantum numbers $\alpha$. The operators $\hat{c}_{\alpha}^{\dagger}$ create electrons in the corresponding eigenstates $|\alpha\rangle$. If now the time-dependent part of the field is switched on, the Hamiltonian becomes $\hat{H}=\hat{H}_{0}+\hat{V}(t)$, with

$$
\hat{V}(t)=\frac{2 \pi}{m L} \delta \varphi(t) \sum_{\alpha, \beta}\left\langle\alpha\left|\hat{P}_{x}(\varphi)\right| \beta\right\rangle \hat{c}_{\alpha}^{\dagger} \hat{c}_{\beta}+\frac{1}{2 m}\left(\frac{2 \pi}{L} \delta \varphi(t)\right)^{2} \sum_{\alpha} \hat{c}_{\alpha}^{\dagger} \hat{c}_{\alpha}
$$

Here $\delta \varphi(t)=\left(\phi_{\varepsilon} / \phi_{0}\right) \sin (\varepsilon t)$, and $\hat{P}_{x}(\varphi)=-i d / d x+(2 \pi / L) \varphi$ is the $x$-component of the one-particle momentum operator. $\hat{V}$ is diagonal in momentum space with matrix elements

$$
\left\langle\mathbf{p}|\hat{V}(t)| \mathbf{p}^{\prime}\right\rangle=\delta_{\mathbf{p}, \mathbf{p}^{\prime}}\left[\frac{2 \pi}{m L} \delta \varphi(t) p_{x}(\varphi)+\frac{1}{2 m}\left(\frac{2 \pi}{L} \delta \varphi(t)\right)^{2}\right],
$$

where $p_{x}(\varphi)$ is the $x$-component of $\mathbf{p}(\varphi)$. The spatially averaged current around the ring is given by $I(t)=\left(a^{2} / \mathcal{V}\right) j_{x}(\mathbf{k}=\mathbf{0}, t)$, where $a^{2}$ is the cross section of the ring and $j_{x}$ is the $x$-component of the current density. From Eqs.(A.3),(A.11) one obtains the paramagnetic contribution

$$
I_{\text {para }}(t)=-\frac{e}{m L} \sum_{\mathbf{q}} q_{x}(\varphi) g_{\mathbf{q}, \mathbf{q}}(t) .
$$


Using the result for the $n$-th order contribution to $g$ (see Eq.(A.17)) yields up to second order in $V$ a paramagnetic current $I_{\text {para }}(t)=I_{\text {para }, 1}+I_{\text {para }, 2}+\mathcal{O}\left(V^{3}\right)$ with

$$
\begin{aligned}
& I_{\text {para }, 1}(t)=-\frac{e}{m L} \sum_{\mathbf{q}, \mathbf{p}} q_{x}(\varphi) \int \frac{d \omega_{1}}{2 \pi} e^{-i \omega_{1} t} V_{\mathbf{p}, \mathbf{p}}\left(\omega_{1}\right) \mathcal{K}^{(1)}\left(\omega_{1}\right) \\
& I_{\text {para }, 2}(t)=-\frac{e}{m L} \sum_{\mathbf{q}, \mathbf{p}, \mathbf{k}} q_{x}(\varphi) \int \frac{d \omega_{1} d \omega_{2}}{(2 \pi)^{2}} e^{-i\left(\omega_{1}+\omega_{2}\right) t} V_{\mathbf{p}, \mathbf{p}}\left(\omega_{1}\right) V_{\mathbf{k}, \mathbf{k}}\left(\omega_{2}\right) \mathcal{K}^{(2)}\left(\omega_{1}, \omega_{2}\right) .
\end{aligned}
$$

The second order in the time dependent perturbation $\delta \varphi(t)$ is thus given by $I^{(2)}(t)=$ $I_{\text {para }, 1}^{(2)}+I_{\text {para }, 2}^{(2)}$ and

$$
\begin{aligned}
& I_{\text {para }, 1}^{(2)}(t)=-\frac{e}{m L} \sum_{\mathbf{q}, \mathbf{p}} q_{x}(\varphi) \int \frac{d \omega_{1}}{2 \pi} e^{-i \omega_{1} t} \frac{1}{2 m}\left(\frac{2 \pi}{L}\right)^{2} \delta \varphi_{\omega_{1}}^{2} \mathcal{K}^{(1)}\left(\omega_{1}\right) \\
& I_{\text {para }, 2}^{(2)}(t)=-\frac{e}{m L} \sum_{\mathbf{q}, \mathbf{p}, \mathbf{k}} q_{x}(\varphi) p_{x}(\varphi) k_{x}(\varphi) \int \frac{d \omega_{1} d \omega_{2}}{(2 \pi)^{2}} e^{-i\left(\omega_{1}+\omega_{2}\right) t}\left(\frac{2 \pi}{m L}\right)^{2} \varphi_{\omega_{1}} \varphi_{\omega_{2}} \mathcal{K}^{(2)}\left(\omega_{1}, \omega_{2}\right)
\end{aligned}
$$

where $\delta \varphi_{\omega}$ is the Fourier transform of $\delta \varphi(t)$, i.e. the time-dependent part of the flux (4.1) in units of the flux quantum, and $\delta \varphi_{\omega}^{2}$ is the Fourier transform of $[\delta \varphi(t)]^{2} . \mathcal{K}^{(1)}$ and $\mathcal{K}^{(2)}$ are obtained from the general expression in Eq.(A.18),

$$
\begin{aligned}
& \mathcal{K}^{(1)}\left(\omega_{1}\right)=\frac{1}{2 i} \int_{-\infty}^{\infty} \frac{d \varepsilon}{2 \pi} \operatorname{Sp}\left[\underline{\sigma}_{1} \underline{G}_{\mathbf{q}, \mathbf{p}}^{0}\left(\varepsilon+\omega_{1}\right) \underline{G}_{\mathbf{p}, \mathbf{q}}^{0}(\varepsilon)\right], \\
& \mathcal{K}^{(2)}\left(\omega_{1}, \omega_{2}\right)=\frac{1}{2 i} \int_{-\infty}^{\infty} \frac{d \varepsilon}{2 \pi} \operatorname{Sp}\left[\underline{\sigma}_{1} \underline{G}_{\mathbf{q}, \mathbf{k}}^{0}\left(\varepsilon+\omega_{1}+\omega_{2}\right) \underline{G}_{\mathbf{k}, \mathbf{p}}^{0}\left(\varepsilon+\omega_{1}\right) \underline{G}_{\mathbf{p}, \mathbf{q}}^{0}(\varepsilon)\right] .
\end{aligned}
$$

Evaluating the trace and utilizing Eq. (A.10) yields the response functions in terms of retarded and advanced momentum-space Green's functions. This representation is convenient for diagrammatic calculations, where one effectively assumes a continuous spectrum. For isolated finite systems in the absence of any inelastic processes (like the one considered here), this procedure overlooks some difficulties arising from the discrete nature of the energy spectrum. To see this, one represents the response functions in the exact eigenstates of the unperturbed system by using

$$
\mathcal{G}_{\mathbf{p}, \mathbf{q}}^{R / A}(\omega)=\sum_{\alpha} \frac{\langle\mathbf{p} \mid \alpha\rangle\langle\alpha \mid \mathbf{q}\rangle}{\omega-\varepsilon_{\alpha} \pm i 0} .
$$

With the formal identity $\operatorname{Im}\left(\omega-\varepsilon_{\alpha}+i 0\right)^{-1}=-\pi \delta\left(\omega-\varepsilon_{\alpha}\right)$ one arrives at

$$
I_{\text {para }, 1}^{(2)}(t)=-\frac{e}{2}\left(\frac{2 \pi}{m L}\right)^{2} \int_{-\infty}^{\infty} \frac{d \omega_{1}}{2 \pi} e^{-i \omega_{1} t} \delta \varphi_{\omega_{1}}^{2} \sum_{\alpha \beta}\left\langle\alpha\left|\hat{P}_{x}(\varphi)\right| \beta\right\rangle\langle\alpha \mid \beta\rangle \frac{f\left(\varepsilon_{\beta}\right)-f\left(\varepsilon_{\alpha}\right)}{\varepsilon_{\beta}-\varepsilon_{\alpha}+\omega_{1}+i 0} .
$$


Here $f\left(\varepsilon_{\alpha}\right)=\left\langle\hat{c}_{\alpha}^{\dagger} \hat{c}_{\alpha}\right\rangle$ is the occupation number, which in a grand-canonical ensemble is the Fermi function. In deriving Eq. (4.11) it was made use of $q_{x}(\varphi)\langle\alpha \mid \mathbf{q}\rangle=$ $\left\langle\alpha\left|\hat{P}_{x}(\varphi)\right| \mathbf{q}\right\rangle$ and $\sum_{\mathbf{q}}|\mathbf{q}\rangle\langle\mathbf{q}|=1$. Eq.(4.11) contains one current vertex and one density vertex. Due to the orthogonality of eigenstates $\langle\alpha \mid \beta\rangle=\delta_{\alpha, \beta}$ the integrand vanishes identically. The second order diamagnetic current $I_{d i a}^{(2)}$ which wasn't addressed so far, has a similar structure as $I_{\text {para }, 1}^{(2)}$. It also contains only one current vertex and one density vertex and vanishes exactly just like $I_{\text {para }, 1}^{(2)}[54]$. The only remaining contribution to the total second order current $I^{(2)}(t)=I_{\text {para }, 1}^{(2)}+I_{\text {para }, 2}^{(2)}+I_{\text {dia }}^{(2)}$ thus comes from $I_{\text {para }, 2}^{(2)}$ and contains three current vertices. A straightforward evaluation of the second order response kernel $\mathcal{K}^{(2)}$ finally yields

$$
I^{(2)}(t)=\frac{(-e)(2 \pi)^{2}}{(m L)^{3}} \int_{-\infty}^{\infty} d \omega_{1} d \omega_{2} \delta \varphi_{\omega_{1}} \delta \varphi_{\omega_{2}} K^{(2)}\left(\omega_{1}, \omega_{2}\right) e^{-i\left(\omega_{1}+\omega_{2}\right) t}
$$

with

$$
\begin{aligned}
& K^{(2)}\left(\omega_{1}, \omega_{2}\right)=\sum_{\alpha \beta \gamma} \frac{P_{\alpha \beta \gamma}}{\varepsilon_{\gamma}-\varepsilon_{\alpha}+\omega_{1}+\omega_{2}+i 0} \\
& \times\left[\frac{f\left(\varepsilon_{\gamma}\right)-f\left(\varepsilon_{\beta}\right)}{\varepsilon_{\gamma}-\varepsilon_{\beta}+\omega_{2}+i 0}-\frac{f\left(\varepsilon_{\beta}\right)-f\left(\varepsilon_{\alpha}\right)}{\varepsilon_{\beta}-\varepsilon_{\alpha}+\omega_{1}+i 0}\right],
\end{aligned}
$$

where $P_{\alpha \beta \gamma}$ is defined as

$$
P_{\alpha \beta \gamma}=\left\langle\alpha\left|\hat{P}_{x}(\varphi)\right| \beta\right\rangle\left\langle\beta\left|\hat{P}_{x}(\varphi)\right| \gamma\right\rangle\left\langle\gamma\left|\hat{P}_{x}(\varphi)\right| \alpha\right\rangle
$$

In contrast to $\mathcal{K}^{(2)}$ the matrix elements $P_{\alpha \beta \gamma}$ are now included in the definition of the response function $K^{(2)}$. This result is valid for a grand canonical as well as for a canonical ensemble. In the latter case the chemical potential $\mu$ is a function of flux and disorder. Thus formally there is no difference in the response functions for the two ensembles. The actual discrepancy due to the condition of a fixed particle number on the one side and fixed $\mu$ on the other is however hard to estimate. It is emphasized, that Eq.(4.13) is the only non-vanishing contribution to the total second order response function, including all diamagnetic and paramagnetic terms [54]. Keeping in mind that the time-dependent part of the flux (4.1) corresponds to

$$
\delta \varphi_{\omega^{\prime}}=\frac{\phi_{\varepsilon}}{2 i \phi_{0}}\left[\delta\left(\omega^{\prime}+\varepsilon\right)-\delta\left(\omega^{\prime}-\varepsilon\right)\right]
$$

it is clear that in this case Eq.(4.12) contains not only oscillating terms, but also a time-independent contribution,

$$
I_{0}^{(2)}=A_{\varepsilon}\left[K^{(2)}(\varepsilon,-\varepsilon)+K^{(2)}(-\varepsilon, \varepsilon)\right],
$$


where

$$
A_{\varepsilon}=\frac{(-e)\left(2 \pi \phi_{\varepsilon}\right)^{2}}{4(L m)^{3} \phi_{0}^{2}}
$$

and

$$
K^{(2)}(\varepsilon,-\varepsilon)=\sum_{\alpha \beta \gamma} \frac{P_{\alpha \beta \gamma}}{\varepsilon_{\gamma}-\varepsilon_{\alpha}+i 0}\left[\frac{f\left(\varepsilon_{\gamma}\right)-f\left(\varepsilon_{\beta}\right)}{\varepsilon_{\gamma}-\varepsilon_{\beta}-\varepsilon+i 0}-\frac{f\left(\varepsilon_{\beta}\right)-f\left(\varepsilon_{\alpha}\right)}{\varepsilon_{\beta}-\varepsilon_{\alpha}+\varepsilon+i 0}\right] .
$$

Defining retarded and advanced Green's functions,

$$
\mathcal{G}_{\alpha}^{R}(\omega)=\frac{1}{\omega-\varepsilon_{\alpha}+i 0}, \mathcal{G}_{\alpha}^{A}(\omega)=\frac{1}{\omega-\varepsilon_{\alpha}-i 0},
$$

Eq.(4.18) can also be written as

$$
\begin{aligned}
K^{(2)}(\varepsilon,-\varepsilon) & =-\frac{1}{2 \pi i} \sum_{\alpha \beta \gamma} P_{\alpha \beta \gamma}\left\{\int _ { - \infty } ^ { \infty } d \omega f ( \omega + \varepsilon ) \left[\mathcal{G}_{\alpha}^{R}(\omega+\varepsilon) \mathcal{G}_{\beta}^{R}(\omega) \mathcal{G}_{\gamma}^{R}(\omega+\varepsilon)\right.\right. \\
& \left.-\mathcal{G}_{\alpha}^{A}(\omega+\varepsilon) \mathcal{G}_{\beta}^{A}(\omega) \mathcal{G}_{\gamma}^{A}(\omega+\varepsilon)\right]-\int_{-\infty}^{\infty} d \omega[f(\omega+\varepsilon)-f(\omega)] \\
& \left.\times\left[\mathcal{G}_{\alpha}^{R}(\omega+\varepsilon) \mathcal{G}_{\beta}^{A}(\omega) \mathcal{G}_{\gamma}^{A}(\omega+\varepsilon)-\mathcal{G}_{\alpha}^{R}(\omega+\varepsilon) \mathcal{G}_{\beta}^{R}(\omega) \mathcal{G}_{\gamma}^{A}(\omega+\varepsilon)\right]\right\}
\end{aligned}
$$

The structure of the Green's functions agrees with the one given by KY in Ref. 62]. Note, however, that these authors work in a different gauge: they represent the electric field by a scalar potential, so that their expressions contain only a single current vertex. The introduction of Green's function is useful for calculating disorder averages. It is common wisdom that for the calculation of the disorder average of Eq.(4.20) the terms involving products of only retarded or only advanced Green's functions can be neglected In this approximation a perturbative calculation of the disorder average of Eq.(4.20) has been given by KY [56], with the result that the associated time-independent part of the non-equilibrium current is proportional to $\varepsilon^{-2}$ for frequencies larger than the Thouless energy. As explained in Sec.4.1, for frequencies $\varepsilon<E_{c}$ the perturbative expansion can not be expected to be valid. In fact, it will turn out, that the physical behavior is completely different for systems with discrete spectrum.

To demonstrate the breakdown of the diagrammatic perturbation theory in the discrete spectrum limit, it is now shown that an exact evaluation of the disorder average of Eq.(4.20) should actually yield an infinite result. One should therefore

\footnotetext{
${ }^{1}$ Note, however, that in a canonical ensemble the chemical potential fluctuates, so that disorder averages involving only retarded or only advanced Green's functions can also generate large contributions to response functions, see Ref. [54].
} 
reconsider the exact spectral representation (4.18) of the response function. Using the formal identity

$$
\frac{1}{x+i 0}=\wp \frac{1}{x}-i \pi \delta(x)
$$

where $\wp$ denotes the Cauchy principal part, one can rewrite Eq.(4.18) as

$$
K^{(2)}(\varepsilon,-\varepsilon)=K_{\wp}^{(2)}(\varepsilon,-\varepsilon)+K_{\delta \delta}^{(2)}(\varepsilon,-\varepsilon),
$$

with

$$
\begin{aligned}
& K_{\wp}^{(2)}(\varepsilon,-\varepsilon)=2 \sum_{\alpha \beta \gamma} \frac{\operatorname{Re} P_{\alpha \beta \gamma}}{\varepsilon_{\gamma}-\varepsilon_{\alpha}} \wp \frac{f\left(\varepsilon_{\gamma}\right)-f\left(\varepsilon_{\beta}\right)}{\varepsilon_{\gamma}-\varepsilon_{\beta}-\varepsilon}, \\
& K_{\delta \delta}^{(2)}(\varepsilon,-\varepsilon)=-2 \pi^{2} \sum_{\alpha \beta \gamma} \operatorname{Re} P_{\alpha \beta \gamma}\left[f\left(\varepsilon_{\gamma}\right)-f\left(\varepsilon_{\beta}\right)\right] \delta\left(\varepsilon_{\gamma}-\varepsilon_{\alpha}\right) \delta\left(\varepsilon_{\gamma}-\varepsilon_{\beta}-\varepsilon\right) .
\end{aligned}
$$

The terms with $\alpha=\gamma$ in Eqs. (4.23) and (4.24) yield the following contributions,

$$
\begin{gathered}
K_{\wp, \text { diag }}^{(2)}(\varepsilon,-\varepsilon)=\wp \sum_{\alpha \beta} P_{\alpha \beta \alpha} \frac{\partial}{\partial \varepsilon_{\alpha}} \frac{f\left(\varepsilon_{\alpha}\right)-f\left(\varepsilon_{\beta}\right)}{\varepsilon_{\alpha}-\varepsilon_{\beta}-\varepsilon} \\
=\wp \sum_{\alpha \beta} P_{\alpha \beta \alpha}\left[\frac{\frac{\partial}{\partial \varepsilon_{\alpha}} f\left(\varepsilon_{\alpha}\right)}{\varepsilon_{\alpha}-\varepsilon_{\beta}-\varepsilon}-\frac{f\left(\varepsilon_{\alpha}\right)-f\left(\varepsilon_{\beta}\right)}{\left(\varepsilon_{\alpha}-\varepsilon_{\beta}-\varepsilon\right)^{2}}\right], \\
K_{\delta \delta, \text { diag }}^{(2)}(\varepsilon,-\varepsilon)=-2 \pi^{2} \delta(0) \sum_{\alpha \beta} \operatorname{Re} P_{\alpha \beta \alpha}\left[f\left(\varepsilon_{\alpha}\right)-f\left(\varepsilon_{\beta}\right)\right] \delta\left(\varepsilon_{\alpha}-\varepsilon_{\beta}-\varepsilon\right) .
\end{gathered}
$$

The right-hand side of Eq.(4.26) is proportional to the infinite factor $\delta(0)$. Hence, the term $K_{\delta \delta}^{(2)}(\varepsilon,-\varepsilon)$ must also be infinite. Because the singular prefactor $\delta(0)$ in Eq. (4.26) does not depend on the disorder, this singularity survives disorder averaging. Keeping in mind that Eq.(4.20) is mathematically equivalent with Eq.(4.18), it is concluded that a correct evaluation of the disorder average $\overline{K^{(2)}(\varepsilon,-\varepsilon)}$ must yield an infinite result?. Unfortunately, in an approximate evaluation of Eq.(4.20) by means of the usual diagrammatic methods this $\delta$-function singularity is artificially smoothed out, and one obtains a finite result [56]. The singular contribution in Eq.(4.26) arises for finite frequencies $\varepsilon>0$. On the other hand, the limit $\lim _{\omega_{1}, \omega_{2} \rightarrow 0} K^{(2)}\left(\omega_{1}, \omega_{2}\right)$ exists and is related to the flux-derivative of the persistent current $[54]$.

\footnotetext{
${ }^{2}$ The same type of singularity occurs if one calculates the disorder average of the square of the density of states, $\overline{\nu_{d}^{2}(\epsilon)}=\frac{1}{L^{2 d}} \overline{\sum_{\alpha \beta} \delta\left(\varepsilon-\varepsilon_{\alpha}\right) \delta\left(\varepsilon-\varepsilon_{\beta}\right)}$. The contribution from the terms with $\alpha=\beta$ is proportional to $\delta(0)=\infty$. Yet, perturbative averaging with the help of the impurity diagram technique produces a finite result.
} 


\subsection{Adiabatic switching on}

The infinite term (4.26) is clearly unphysical. This term is closely related to the infinitesimal imaginary parts $i 0$ that have been added to the real frequencies in the spectral representation (4.18) for the response function $K^{(2)}(\varepsilon,-\varepsilon)$. As emphasized by KY [62], the infinitesimal imaginary parts are a consequence of the fact that the response function must be causal when the time-dependent part of the Hamiltonian is adiabatically switched on. The "adiabatic switching on" of the time-dependent perturbation will now be examined more carefully. Following the usual recipe [63], the Hamiltonian $\hat{H}_{0}+\hat{V}(t)$ is replaced by $\hat{H}_{0}+\hat{V}_{\eta}(t)$, where $\hat{V}_{\eta}(t)=\exp (\eta t) \hat{V}(t)$. The limit $\eta \rightarrow 0$ is then taken at the end of the calculation of physical quantities. For large enough times $t$ the physical result should be independent of the switching on procedure. Indeed, in Sec.4.3.1 it is shown by explicit calculation that sudden switching on produces the same result for the long-time response as adiabatic switching on. However, in the latter case one still has to be careful to take the limit $\eta \rightarrow 0$ only after the physical quantity of interest has been calculated. It is now shown that the singularity in Eq. (4.26) has been artificially generated by taking the limit $\eta \rightarrow 0$ at an intermediate step of the calculation.

By direct expansion of the time evolution operator in the interaction representation to second order in the time-dependent perturbation, one obtains the current for adiabatic switching on at finite $\eta$. Formally the additional factor $\exp (\eta t)$ merely shifts the frequencies according to $\omega_{j} \rightarrow \omega_{j}+i \eta(j=1,2)$, which leads to

$$
I_{\eta}^{(2)}(t)=\frac{(-e)(2 \pi)^{2}}{(m L)^{3}} \int_{-\infty}^{\infty} d \omega_{1} d \omega_{2} \delta \varphi_{\omega_{1}} \delta \varphi_{\omega_{2}} K_{\eta t}^{(2)}\left(\omega_{1}, \omega_{2}\right) e^{-i\left(\omega_{1}+\omega_{2}\right) t}
$$

with

$$
\begin{array}{r}
K_{\eta t}^{(2)}\left(\omega_{1}, \omega_{2}\right)=e^{2 \eta t} \sum_{\alpha \beta \gamma} \frac{P_{\alpha \beta \gamma}}{\varepsilon_{\gamma}-\varepsilon_{\alpha}+\omega_{1}+\omega_{2}+2 i \eta} \\
\times\left[\frac{f\left(\varepsilon_{\gamma}\right)-f\left(\varepsilon_{\beta}\right)}{\varepsilon_{\gamma}-\varepsilon_{\beta}+\omega_{2}+i \eta}-\frac{f\left(\varepsilon_{\beta}\right)-f\left(\varepsilon_{\alpha}\right)}{\varepsilon_{\beta}-\varepsilon_{\alpha}+\omega_{1}+i \eta}\right] .
\end{array}
$$

Comparing Eq.(4.28) with Eq.(4.13), one sees that the former is multiplied by an extra factor of $e^{2 \eta t}$. If one directly takes the limit $\eta \rightarrow 0$, this factor is replaced by unity. This is the limiting procedure adopted in the usual Green's function approach, where one takes first the limit $\eta \rightarrow 0$ in Eq.(4.28) and then inserts the resulting expression into Eq.(4.27). In this case Eqs.(4.16) and (4.18) are recovered, which lead to the divergence in Eq.(4.26). It is now shown that this unphysical divergence does not appear if the limit $\eta \rightarrow 0$ is taken after the physical current has been calculated. Substituting Eq.(4.28) into Eq.(4.27) one obtains

$$
\begin{aligned}
I_{\eta}^{(2)}(t)= & A_{\varepsilon}\left[K_{\eta t}^{(2)}(\varepsilon,-\varepsilon)+K_{\eta t}^{(2)}(-\varepsilon, \varepsilon)\right. \\
& \left.+K_{\eta t}^{(2)}(\varepsilon, \varepsilon) e^{-2 i \varepsilon t}+K_{\eta t}^{(2)}(-\varepsilon,-\varepsilon) e^{2 i \varepsilon t}\right] .
\end{aligned}
$$


In analogy with Eq. (4.22), $K_{\eta t}^{(2)}(\varepsilon,-\varepsilon)$ is expressed in terms of products of real and imaginary parts

$$
K_{\eta t}^{(2)}(\varepsilon,-\varepsilon)=K_{\eta t, \wp}^{(2)}(\varepsilon,-\varepsilon)+K_{\eta t, \delta \delta}^{(2)}(\varepsilon,-\varepsilon)
$$

with

$$
\begin{array}{r}
K_{\eta t, \wp}^{(2)}(\varepsilon,-\varepsilon)=2 e^{2 \eta t} \sum_{\alpha \beta \gamma} \operatorname{Re} P_{\alpha \beta \gamma}\left[f\left(\varepsilon_{\gamma}\right)-f\left(\varepsilon_{\beta}\right)\right] \\
\times\left[\frac{\varepsilon_{\gamma}-\varepsilon_{\alpha}}{\left(\varepsilon_{\gamma}-\varepsilon_{\alpha}\right)^{2}+(2 \eta)^{2}} \frac{\varepsilon_{\gamma}-\varepsilon_{\beta}-\varepsilon}{\left(\varepsilon_{\gamma}-\varepsilon_{\beta}-\varepsilon\right)^{2}+\eta^{2}}\right], \\
K_{\eta t, \delta \delta}^{(2)}(\varepsilon,-\varepsilon)=-2 e^{2 \eta t} \sum_{\alpha \beta \gamma} \operatorname{Re} P_{\alpha \beta \gamma}\left[f\left(\varepsilon_{\gamma}\right)-f\left(\varepsilon_{\beta}\right)\right] \\
\times\left[\frac{2 \eta}{\left(\varepsilon_{\gamma}-\varepsilon_{\alpha}\right)^{2}+(2 \eta)^{2}} \frac{\eta}{\left(\varepsilon_{\gamma}-\varepsilon_{\beta}-\varepsilon\right)^{2}+\eta^{2}}\right] .
\end{array}
$$

From Eq.(4.31) it is now obvious that $K_{\eta t, \wp}^{(2)}$ does not have any contributions from the terms $\alpha=\gamma$. The finite contribution in Eq.(4.25) is thus an artefact of taking the limit $\eta \rightarrow 0$ before calculating any physical quantities. Now the term (4.32) is closer investigated. By directly taking the limit $\eta \rightarrow 0$ using

$$
\lim _{\eta \rightarrow 0} \frac{\eta}{\epsilon^{2}+\eta^{2}}=\pi \delta(\epsilon)
$$

the infinite result (4.26) is recovered. However, the structure of the $\eta$-dependent part of Eq.(4.32) is familiar from the derivation of Fermi's golden rule of elementary quantum mechanics. As discussed for example in the classic textbook by Baym [63], terms with this structure should be interpreted as a rate, i.e. as a contribution to the current that grows linearly in time. It is therefore clear that after taking the derivative of Eq.(4.32) with respect to $t$ one obtains a finite result when performing the limit $\eta \rightarrow 0$. A simple calculation yields

$$
\begin{aligned}
\lim _{\eta \rightarrow 0} \frac{d}{d t} K_{\eta t, \delta \delta}^{(2)}(\varepsilon,-\varepsilon) & =-2 \lim _{\eta \rightarrow 0} \sum_{\alpha \beta} P_{\alpha \beta \alpha} \frac{\left[f\left(\varepsilon_{\alpha}\right)-f\left(\varepsilon_{\beta}\right)\right] \eta}{\left(\varepsilon_{\alpha}-\varepsilon_{\beta}-\varepsilon\right)^{2}+\eta^{2}} \\
& =-2 \pi \sum_{\alpha \beta} P_{\alpha \beta \alpha}\left[f\left(\varepsilon_{\alpha}\right)-f\left(\varepsilon_{\beta}\right)\right] \delta\left(\varepsilon_{\alpha}-\varepsilon_{\beta}-\varepsilon\right) .
\end{aligned}
$$

Because this expression contains only a single $\delta$-function, after averaging over disorder it becomes a smooth function of $\varepsilon$. Since the above derivation relies on Fermi's golden rule, it is valid for times $t<1 / \Delta$ [63]. Together with the condition $1 / \tau_{e e}<\Delta$ for a discrete spectrum it follows, that the linear time dependence of the current should be observable on time scales $t<\Delta^{-1}<\tau_{e e}$. In conclusion, to quadratic order 
in the field the non-equilibrium current induced by the time-dependent flux (4.1) has the following three contributions,

$$
I^{(2)}(t) \equiv \lim _{\eta \rightarrow 0} I_{\eta}^{(2)}(t)=I_{t h}^{(2)}+t \frac{d I_{k i n}^{(2)}}{d t}+I_{o s c}^{(2)}(t)
$$

where the time-independent part is given by

$$
\begin{aligned}
I_{t h}^{(2)} & =A_{\varepsilon} \lim _{\eta \rightarrow 0}\left[K_{\eta t, \wp}^{(2)}(\varepsilon,-\varepsilon)+K_{\eta t, \wp}^{(2)}(-\varepsilon, \varepsilon)\right] \\
& =2 A_{\varepsilon} \sum_{\alpha \beta \gamma, \alpha \neq \gamma} \frac{\operatorname{Re} P_{\alpha \beta \gamma}}{\varepsilon_{\gamma}-\varepsilon_{\alpha}} \wp\left[\frac{f\left(\varepsilon_{\gamma}\right)-f\left(\varepsilon_{\beta}\right)}{\varepsilon_{\gamma}-\varepsilon_{\beta}-\varepsilon}+(\varepsilon \rightarrow-\varepsilon)\right] .
\end{aligned}
$$

The coefficient of the term linear in time is

$$
\begin{aligned}
\frac{d I_{k i n}^{(2)}}{d t} & =A_{\varepsilon} \lim _{\eta \rightarrow 0}\left[\frac{d}{d t} K_{\eta t, \delta \delta}^{(2)}(\varepsilon,-\varepsilon)+\frac{d}{d t} K_{\eta t, \delta \delta}^{(2)}(-\varepsilon, \varepsilon)\right] \\
& =-2 \pi A_{\varepsilon} \sum_{\alpha \beta} P_{\alpha \beta \alpha}\left[f\left(\varepsilon_{\alpha}\right)-f\left(\varepsilon_{\beta}\right)\right]\left[\delta\left(\varepsilon_{\alpha}-\varepsilon_{\beta}-\varepsilon\right)+(\varepsilon \rightarrow-\varepsilon)\right],
\end{aligned}
$$

and the oscillating part is

$$
I_{o s c}^{(2)}(t)=-A_{\varepsilon} \lim _{\eta \rightarrow 0}\left[K_{\eta t}^{(2)}(\varepsilon, \varepsilon) e^{-2 i \varepsilon t}+K_{\eta t}^{(2)}(-\varepsilon,-\varepsilon) e^{2 i \varepsilon t}\right] .
$$

Thus, a time-dependent electric field with frequency $\varepsilon$ induces in quadratic order three fundamentally different currents. (a) A time-independent contribution $I_{t h}^{(2)}$. (b) A contribution $t d I_{\text {kin }}^{(2)} / d t$ which increases linearly in time; this term can be understood in terms of the usual golden rule of time-dependent perturbation theory.

(c) Finally, there is also a time-dependent contribution $I_{\text {osc }}^{(2)}$ oscillating with frequency $2 \varepsilon$. When this term is averaged over a time-interval larger than $\varepsilon^{-1}$, its contribution to the current is negligible small.

From the above analysis it is clear that the contribution that is proportional to $t$ cannot be calculated within the usual Green's function machinery, because in this approach the limit $\eta \rightarrow 0$ is taken at an intermediate step of the calculation, causing an unphysical divergence. To further support the correctness of the limiting procedure adopted here it is shown in the next section that Eqs.(4.35)-(4.37) can also be re-derived if the perturbation is suddenly (instead of adiabatically) switched on.

\subsubsection{Comparison with sudden switching on}

To confirm that the "switching on procedure" outlined in Sec.4.3 yields the correct physical results, a harmonic perturbation that is suddenly turned on at time $t=0$ is considered for comparison:

$$
\phi(t)=\phi+\phi_{\varepsilon} \Theta(t) \sin (\varepsilon t),
$$


where $\Theta(t)$ is the step function. In the long time limit the physical behavior should be independent of the switching on procedure. The paramagnetic current is evaluated from the general expression

$$
I_{\text {para }}=-\frac{e}{m L} \sum_{\mathbf{q}} q_{x}(\varphi)\left\langle\hat{\Psi}_{H, \mathbf{q}}^{\dagger}(t) \hat{\Psi}_{H, \mathbf{q}}(t)\right\rangle .
$$

Changing from Heisenberg to Dirac picture and from momentum to energy representation Eq.(4.40) becomes

$$
I_{\text {para }}=-\frac{e}{m L} \sum_{\alpha \beta} P_{\alpha \beta}\left\langle\hat{U}_{D}^{\dagger}(t) \hat{c}_{D, \alpha}^{\dagger}(t) \hat{c}_{D, \beta}(t) \hat{U}_{D}(t)\right\rangle,
$$

where $P_{\alpha \beta}=\left\langle\alpha\left|\hat{P}_{x}(\varphi)\right| \beta\right\rangle$ and the time-evolution operator is given by

$$
\hat{U}_{D}(t)=\sum_{n=0}^{\infty} \frac{(-i)^{n}}{n !} \int_{-\infty}^{t} d t_{1} \cdots \int_{-\infty}^{t} d t_{n} \hat{T}\left[\hat{V}_{D}\left(t_{1}\right) \cdots \hat{V}_{D}\left(t_{n}\right)\right] .
$$

$\hat{V}_{D}(t)$ is the Dirac picture of the external perturbation given in Eq.(4.4). The index $D$ will be suppressed in the following. To first order in $\delta \varphi(t)=\left(\phi / \phi_{0}\right) \Theta(t) \sin (\varepsilon t)$ one obtains

$$
\begin{array}{r}
I_{\text {para }}^{(1)}(t)=-\frac{e i}{m L} \int_{0}^{t} d t_{1} \frac{2 \pi}{m L} \delta \varphi\left(t_{1}\right) \sum_{\alpha \beta \gamma \delta} P_{\alpha \beta} P_{\gamma \delta}\left[\left\langle\hat{c}_{\alpha}^{\dagger}\left(t_{1}\right) \hat{c}_{\beta}\left(t_{1}\right) \hat{c}_{\gamma}^{\dagger}(t) \hat{c}_{\delta}(t)\right\rangle\right. \\
\left.-\left\langle\hat{c}_{\gamma}^{\dagger}(t) \hat{c}_{\delta}(t) \hat{c}_{\alpha}^{\dagger}\left(t_{1}\right) \hat{c}_{\beta}\left(t_{1}\right)\right\rangle\right] .
\end{array}
$$

Since $H_{0}=\sum_{\alpha} \varepsilon_{\alpha} \hat{c}_{\alpha}^{\dagger} \hat{c}_{\alpha}$ the time dependence of the operators is trivial:

$$
\hat{c}_{\alpha}^{\dagger}\left(t_{1}\right)=e^{i \varepsilon_{\alpha} t_{1}} \hat{c}_{\alpha} ; \hat{c}_{\beta}\left(t_{1}\right)=e^{-i \varepsilon_{\beta} t_{1}} \hat{c}_{\beta} .
$$

Decomposing the expectation values by the Wick theorem and using $\left\langle\hat{c}_{\alpha}^{\dagger} \hat{c}_{\beta}\right\rangle=$ $\delta_{\alpha, \beta} f\left(\varepsilon_{\alpha}\right)$ as well as $\hat{c}_{\alpha}^{\dagger} \hat{c}_{\beta}+\hat{c}_{\beta} \hat{c}_{\alpha}^{\dagger}=\delta_{\alpha, \beta}$ finally yields

$$
I_{\text {para }}^{(1)}(t)=-\frac{2 \pi i e}{(m L)^{2}} \int_{0}^{t} d t_{1} \delta \varphi\left(t_{1}\right) \sum_{\alpha \beta}\left|P_{\alpha \beta}\right|^{2} e^{i\left(\varepsilon_{\alpha}-\varepsilon_{\beta}\right)\left(t_{1}-t\right)}\left[f\left(\varepsilon_{\alpha}\right)-f\left(\varepsilon_{\beta}\right)\right] .
$$

Obviously there is no contribution from the diagonal term with $\alpha=\beta$. Now the explicit form of $\delta \varphi(t)$ can be inserted:

$$
\begin{gathered}
I_{\text {para }}^{(1)}(t)=-\frac{\pi e}{(m L)^{2}} \frac{\phi_{\varepsilon}}{\phi_{0}} \int_{0}^{t} d t_{1}\left(e^{i \varepsilon t}-e^{-i \varepsilon t}\right) \sum_{\alpha \beta ; \alpha \neq \beta}\left|P_{\alpha \beta}\right|^{2} e^{i\left(\varepsilon_{\alpha}-\varepsilon_{\beta}\right)\left(t_{1}-t\right)}\left[f\left(\varepsilon_{\alpha}\right)-f\left(\varepsilon_{\beta}\right)\right] \\
=-\frac{\pi e}{i(m L)^{2}} \frac{\phi_{\varepsilon}}{\phi_{0}} \sum_{\alpha \beta ; \alpha \neq \beta}\left|P_{\alpha \beta}\right|^{2}\left[f\left(\varepsilon_{\alpha}\right)-f\left(\varepsilon_{\beta}\right)\right]\left[\frac{e^{i \varepsilon t}-e^{-i\left(\varepsilon_{\alpha}-\varepsilon_{\beta}\right) t}}{\varepsilon_{\alpha}-\varepsilon_{\beta}+\varepsilon}\right. \\
\left.-\frac{e^{-i \varepsilon t}-e^{-i\left(\varepsilon_{\alpha}-\varepsilon_{\beta}\right) t}}{\varepsilon_{\alpha}-\varepsilon_{\beta}-\varepsilon}\right] .
\end{gathered}
$$


After averaging over a time interval $t \gtrsim \varepsilon^{-1}$ this current vanishes.

The second order in $\phi_{\varepsilon}$ is more interesting. Since the calculation is not more difficult than for the linear response but much more lengthy, here only the result is stated:

$$
\begin{aligned}
I_{\text {para }}^{(2)}(t) & =2 A_{\varepsilon} \operatorname{Re} \sum_{\alpha \beta \gamma} P_{\alpha \beta \gamma}\left[f\left(\varepsilon_{\beta}\right)-f\left(\varepsilon_{\alpha}\right)\right]\left\{\frac{e^{2 i \varepsilon t}-e^{i\left(\varepsilon_{\gamma}-\varepsilon_{\alpha}\right) t}}{\left(\varepsilon_{\alpha}-\varepsilon_{\gamma}+2 \varepsilon\right)\left(\varepsilon_{\alpha}-\varepsilon_{\beta}+\varepsilon\right)}\right. \\
& -\frac{1-e^{i\left(\varepsilon_{\gamma}-\varepsilon_{\alpha}\right) t}}{\left(\varepsilon_{\alpha}-\varepsilon_{\gamma}\right)\left(\varepsilon_{\alpha}-\varepsilon_{\beta}-\varepsilon\right)}+\frac{2 \varepsilon}{\left(\varepsilon_{\alpha}-\varepsilon_{\beta}\right)^{2}-\varepsilon^{2}}\left[\frac{e^{i\left(\varepsilon_{\beta}-\varepsilon_{\alpha}+\varepsilon\right) t}-e^{i\left(\varepsilon_{\gamma}-\varepsilon_{\alpha}\right) t}}{\varepsilon_{\beta}-\varepsilon_{\gamma}+\varepsilon}\right] \\
& +(\varepsilon \rightarrow-\varepsilon)\} .
\end{aligned}
$$

The diagonal term $\alpha=\gamma$ is

$$
\begin{aligned}
I_{\text {diag }}^{(2)}(t) & =4 A_{\varepsilon} \sum_{\alpha \beta} P_{\alpha \beta \alpha}\left[f\left(\varepsilon_{\alpha}\right)-f\left(\varepsilon_{\beta}\right)\right]\left[\frac{\sin ^{2}(\varepsilon t)}{\left(\varepsilon_{\alpha}-\varepsilon_{\beta}\right)^{2}-\varepsilon^{2}}\right. \\
& \left.-\frac{\sin ^{2}\left(\frac{\varepsilon_{\beta}-\varepsilon_{\alpha}+\varepsilon}{2} t\right)+\sin ^{2}\left(\frac{\varepsilon_{\beta}-\varepsilon_{\alpha}-\varepsilon}{2} t\right)}{\left(\varepsilon_{\alpha}-\varepsilon_{\beta}\right)^{2}-\varepsilon^{2}}+\left[\frac{\sin \left(\frac{\varepsilon_{\beta}-\varepsilon_{\alpha}+\varepsilon}{2} t\right)}{\varepsilon_{\beta}-\varepsilon_{\alpha}+\varepsilon}\right]^{2}+\left[\frac{\sin \left(\frac{\varepsilon_{\beta}-\varepsilon_{\alpha}-\varepsilon}{2} t\right)}{\varepsilon_{\beta}-\varepsilon_{\alpha}-\varepsilon}\right]^{2}\right] .
\end{aligned}
$$

The last two terms can be interpreted in the same way as is done in Fermi's golden rule 63] by using the identity

$$
\left[\frac{\sin \left(\frac{\Delta \varepsilon}{2} t\right)}{\Delta \varepsilon}\right]^{2} \rightarrow \frac{\pi}{2} t \delta(\Delta \varepsilon) \text { for } t \rightarrow \infty
$$

It is now easy to see that for large times $I_{\text {diag }}^{(2)}(t)$ yields exactly the same linear in time contribution as given in Eq.(4.37). The terms with no explicit time dependence in Eq.(4.47), i.e.

$$
I_{0}^{(2)}=-2 A_{\varepsilon} \operatorname{Re} \sum_{\alpha \beta \gamma} P_{\alpha \beta \gamma}\left[f\left(\varepsilon_{\beta}\right)-f\left(\varepsilon_{\alpha}\right)\right]\left[\frac{1}{\left(\varepsilon_{\alpha}-\varepsilon_{\gamma}\right)\left(\varepsilon_{\alpha}-\varepsilon_{\beta}-\varepsilon\right)}+(\varepsilon \rightarrow-\varepsilon)\right],
$$

can be identified with $I_{t h}^{(2)}$ in Eq.(4.36).

\subsection{Numerical results}

Due to the restrictions $\alpha \neq \gamma$ in Eq.(4.36) and $\alpha=\gamma$ in Eq.(4.37) these terms can not be studied within the framework of the usual diagrammatic Green's function 
technique. Since there are no standard methods to treat such equations, looking for an controlled analytical approximation seem rather complicated. To evaluate Eqs.(4.36), (4.37) one in principle has to calculate all eigenenergies $\varepsilon_{\alpha}$, eigenstates $|\alpha\rangle$ and matrix elements $P_{\alpha \beta \gamma}$ as a functional of the disorder distribution. Such a procedure can of course always be performed for a suitably sized computer model. To perform computer simulations, the system is mapped onto a tight-binding model. Making use of the standard Peierls substitution, the static component of the external flux enters as a phase to the matrix element for hopping along the circumference of the ring. This direction is chosen as the $x$-axis, while the $y$ - and $z$-axis correspond to the transverse directions. The unperturbed, static part of the Hamiltonian becomes

$$
\hat{H}_{0}=-\epsilon_{h} \sum_{\mathbf{r}} \sum_{\delta \mathbf{r}} e^{i \Phi_{\delta \mathbf{r}}}|\mathbf{r}\rangle\left\langle\mathbf{r}+\delta \mathbf{r}\left|+\sum_{\mathbf{r}} U_{\mathbf{r}}\right| \mathbf{r}\right\rangle\langle\mathbf{r}|
$$

Here $\delta \mathbf{r}$ represents the basis vectors of a lattice cell, which for a cubic lattice with lattice distance $a$ means $\delta \mathbf{r}= \pm a \mathbf{e}_{i}, i=x, y, z$, and $\mathbf{e}_{i}$ are normalized basis vectors with $\left\|\mathbf{e}_{i}\right\|=1$. The number of lattice points in each direction will be denoted $N_{i}$. $\epsilon_{h}$ is the hopping matrix element and $U_{\mathbf{r}}$ the on-site disorder potential which is assumed to be uniformly distributed between $-w / 2$ and $w / 2$. The phase acquired by the electron in each hopping is given by $\Phi_{\delta \mathbf{r}}$ as

$$
\Phi_{\delta \mathbf{r}}= \begin{cases} \pm \frac{2 \pi \varphi}{N_{x}} & \text { for } \delta \mathbf{r}= \pm a \mathbf{e}_{x} \\ 0 & \text { else }\end{cases}
$$

and $\varphi=\phi / \phi_{0}$ is the flux in units of the flux quantum $\phi_{0}$. To calculate the current to quadratic order in the field, one needs the matrix elements of the velocity operator $\hat{P}_{x} / m$. From $\partial \hat{H} / \partial \varphi=(2 \pi / L) \hat{P}_{x} / m$, where $L=L_{x}=a N_{x}$, the velocity operator in the tight-binding limit is obtained as

$$
\frac{\hat{P}_{x}}{m}=-i a \epsilon_{h} \sum_{\mathbf{r}}\left(e^{\frac{2 \pi i \varphi}{N x}}|\mathbf{r}\rangle\left\langle\mathbf{r}+a \mathbf{e}_{x}\left|-e^{-\frac{2 \pi i \varphi}{N x}}\right| \mathbf{r}\right\rangle\left\langle\mathbf{r}-a \mathbf{e}_{x}\right|\right) .
$$

In the following the hopping matrix element and the lattice spacing are set equal to unity, $\epsilon_{h}=1, a=1$. Now by diagonalizing the Hamiltonian (4.51) for different values of $\varphi$ and different realizations of disorder, Eq.4.37) and (4.36) can be numerically calculated. To obtain the eigenvalues and eigenvectors, the Lanczos algorithm, which is especially suited for sparse matrices with non degenerate spectrum is used [74]. In disordered systems the energy levels repel each other, so that no multiple eigenvalues occur. In a canonical ensemble, where the particle number is fixed, the chemical potential $\mu$ is a functional of disorder and flux $\varphi$. The analytical calculations by KY were performed for a grand canonical ensemble, assuming for convenience periodic boundary conditions in $y$ - and $z$-direction. Numerically one can also study the influence of the boundary conditions and the statistical ensemble. 
It is convenient to write the contributions of Eq.(4.31) and (4.32) to the current as $I_{\eta t, \wp}^{(2)}=: A_{\varepsilon} m^{3} e^{2 \eta t} G_{\eta, \wp}(\varepsilon, \varphi)$ with

$$
G_{\eta, \wp}(\varepsilon, \varphi)=\frac{e^{-2 \eta t}}{m^{3}}\left[K_{\eta t, \wp}^{(2)}(\varepsilon,-\varepsilon)+K_{\eta t, \wp}^{(2)}(-\varepsilon, \varepsilon)\right]
$$

and $I_{\eta t, \delta \delta}^{(2)}=: A_{\varepsilon} m^{3} e^{2 \eta t} G_{\eta, \delta \delta}(\varepsilon, \varphi)$ with

$$
G_{\eta, \delta \delta}(\varepsilon, \varphi)=\frac{e^{-2 \eta t}}{m^{3}}\left[K_{\eta t, \delta \delta}^{(2)}(\varepsilon,-\varepsilon)+K_{\eta t, \delta \delta}^{(2)}(-\varepsilon, \varepsilon)\right] .
$$

The factor $1 / \mathrm{m}^{3}$ is defined into the function $G$, since numerically matrix elements of the velocity operator $\hat{P} / m$ are evaluated, rather than directly those of $\hat{P}$. According to the analysis in the previous section, $I_{\eta t, \wp}^{(2)}$ should have a finite limit $I_{t h}^{(2)}=\lim _{\eta \rightarrow 0} I_{\eta t, \wp}^{(2)}$ while $I_{\eta t, \delta \delta}^{(2)}$ should diverge for $\eta \rightarrow 0$ and $\varepsilon>0$. These findings will now be tested for the computer model. In the zero temperature limit $T \rightarrow 0$ the occupation number function $f\left(\varepsilon_{\alpha}\right)$ reduces to $\Theta\left(\mu-\varepsilon_{\alpha}\right)$, restricting the range of summation. This yields

$$
\begin{aligned}
G_{\eta, \wp}(\varepsilon, \varphi)=2 \sum_{\alpha=1}^{N_{\text {tot }}} \sum_{\beta=N+1}^{N_{\text {tot }}} \sum_{\gamma=1}^{N} & \operatorname{Re} \frac{P_{\alpha \beta \gamma}}{m^{3}}\left[\frac{\varepsilon_{\gamma}-\varepsilon_{\alpha}}{\left(\varepsilon_{\gamma}-\varepsilon_{\alpha}\right)^{2}+(2 \eta)^{2}}+\frac{\varepsilon_{\beta}-\varepsilon_{\alpha}}{\left(\varepsilon_{\beta}-\varepsilon_{\alpha}\right)^{2}+(2 \eta)^{2}}\right] \times \\
& {\left[\frac{\varepsilon_{\gamma}-\varepsilon_{\beta}-\varepsilon}{\left(\varepsilon_{\gamma}-\varepsilon_{\beta}-\varepsilon\right)^{2}+\eta^{2}}+\frac{\varepsilon_{\gamma}-\varepsilon_{\beta}+\varepsilon}{\left(\varepsilon_{\gamma}-\varepsilon_{\beta}+\varepsilon\right)^{2}+\eta^{2}}\right], }
\end{aligned}
$$

and

$$
\begin{gathered}
G_{\eta, \delta \delta}(\varepsilon, \varphi)=-2 \sum_{\alpha=1}^{N_{\text {tot }}} \sum_{\beta=N+1}^{N_{\text {tot }}} \sum_{\gamma=1}^{N} \operatorname{Re} \frac{P_{\alpha \beta \gamma}}{m^{3}}\left[\frac{2 \eta}{\left(\varepsilon_{\gamma}-\varepsilon_{\alpha}\right)^{2}+(2 \eta)^{2}}+\frac{2 \eta}{\left(\varepsilon_{\beta}-\varepsilon_{\alpha}\right)^{2}+(2 \eta)^{2}}\right] \times \\
\\
{\left[\frac{\eta}{\left(\varepsilon_{\gamma}-\varepsilon_{\beta}-\varepsilon\right)^{2}+\eta^{2}}+\frac{\eta}{\left(\varepsilon_{\gamma}-\varepsilon_{\beta}+\varepsilon\right)^{2}+\eta^{2}}\right] .}
\end{gathered}
$$

$N_{\text {tot }}$ is the total number of states in the system. In the tight-binding model this is equivalent to the number of lattice points, $N_{t o t}=N_{x} N_{y} N_{z} . N=N(\mu)$ is the number of occupied states with energy smaller than the chemical potential $N(\mu)=$ $\sum_{\alpha} \Theta\left(\mu-\varepsilon_{\alpha}\right)$. In a grand canonical ensemble this number of course also depends on disorder and flux. From the symmetry properties $\left\langle\alpha(-\varphi)\left|\hat{P}_{x}(-\varphi)\right| \beta(-\varphi)\right\rangle=$ $-\left\langle\beta(\varphi)\left|\hat{P}_{x}(\varphi)\right| \alpha(\varphi)\right\rangle$ and $\varepsilon_{\alpha}(-\varphi)=\varepsilon_{\alpha}(\varphi)$ it follows directly, that

$$
G_{\eta, \wp / \delta \delta}(\varepsilon, \varphi)=-G_{\eta, \wp / \delta \delta}(\varepsilon,-\varphi) .
$$

To see this, one has to analyze the symmetry of $\operatorname{Re} P_{\alpha \beta \gamma}=\operatorname{Re} P_{\alpha \beta} P_{\beta \gamma} P_{\gamma \alpha}$ with $P_{\alpha \beta}(\varphi)=\left\langle\alpha\left|\hat{P}_{x}(\varphi)\right| \beta\right\rangle$. Representing the complex number $P_{\alpha \beta}$ as the product of absolute value and phase, $P_{\alpha \beta}(\varphi)=\left|P_{\alpha \beta}(\varphi)\right| \exp \left[i \xi_{\alpha \beta}(\varphi)\right]$, it follows from 
$P_{\alpha \beta}(-\varphi)=-P_{\alpha \beta}^{*}(\varphi)\left(*\right.$ denotes the complex conjugate) that $\xi_{\alpha \beta}(-\varphi)=\pi-\xi_{\alpha \beta}(\varphi)$ and $\left|P_{\alpha \beta}(-\varphi)\right|=\left|P_{\alpha \beta}(\varphi)\right|$. Thus

$$
\begin{aligned}
& \operatorname{Re}\left[P_{\alpha \beta}(\varphi) P_{\beta \gamma}(\varphi) P_{\gamma \alpha}(\varphi)\right]=\left|P_{\alpha \beta}(\varphi) P_{\beta \gamma}(\varphi) P_{\gamma \alpha}(\varphi)\right| \cos \left[\xi_{\alpha \beta}(\varphi)+\xi_{\beta \gamma}(\varphi)+\xi_{\gamma \alpha}(\varphi)\right] \\
& =\left|P_{\alpha \beta}(-\varphi) P_{\beta \gamma}(-\varphi) P_{\gamma \alpha}(-\varphi)\right| \cos \left[3 \pi-\xi_{\alpha \beta}(-\varphi)-\xi_{\beta \gamma}(-\varphi)-\xi_{\gamma \alpha}(-\varphi)\right] \\
& =-\operatorname{Re}\left[P_{\alpha \beta}(-\varphi) P_{\beta \gamma}(-\varphi) P_{\gamma \alpha}(-\varphi)\right] .
\end{aligned}
$$

This proofs Eq.(4.58). And since $G_{\eta}$ is necessarily periodic in $\varphi$ with period 1 one has

$$
G_{\eta, \wp / \delta \delta}\left(\varepsilon, \varphi+\frac{1}{2}\right)=G_{\eta, \wp / \delta \delta}\left(\varepsilon, \varphi-\frac{1}{2}\right)=-G_{\eta, \wp / \delta \delta}\left(\varepsilon, \frac{1}{2}-\varphi\right) .
$$

Hence $G_{\eta}$ is symmetric to $\varphi=1 / 2$ and it is sufficient to calculate it for flux values between 0 and $1 / 2$ (this symmetry was also verified numerically). Fig.4.1 shows $G_{\eta, \wp}(\varepsilon, \varphi)$ as a function of flux $\varphi$ for different values of $\varepsilon$ and $\eta$. The calculations were performed for a grand canonical $10 \times 3 \times 3$ lattice with $\mu=0, w=5$ and periodic boundary conditions in $y$ - and $z$-direction. This system has already been studied in Ref. [75] and was found to be diffusive. The band-width of the tight-binding model without disorder reaches from -6 to 6 in the dimensionless numerical units. Disorder smoothens the density of states but leaves the band-width unaffected. For the numerical calculations only the part of the energy spectrum with an approximately constant density of states $\rho(\varepsilon) \approx \Delta^{-1}$ was used, since this is the assumption underlying all theoretical calculations. The importance of this assumption for numerical calculations has been pointed out in Ref. [16]. For the $10 \times 3 \times 3$ system $\rho(\varepsilon)$ was found to be approximately constant for $|\varepsilon| \leq 2$. If not explicitly stated differently, the following numerical results where obtained using this part of the spectrum. For decreasing $\eta$ the number of samples averaged over has to be drastically increased in order to obtain smooth curves. The data for $\eta=10^{-4}$ shows considerable statistical fluctuations even after averaging over 20.000 realizations, but the rather smooth curve for $\eta=10^{-2}$ is still a good fit for these data-points. The numerical results thus confirm the existence of a finite limit $I_{t h}^{(2)}=\lim _{\eta \rightarrow 0} I_{\eta t, \wp \wp}^{(2)}$. In contrast Fig. 4.2 clearly indicates, that no such limit exists for $G_{\eta, \delta \delta}(\varepsilon, \varphi)$ with $\varepsilon>0$. The function grows $\sim 1 / \eta$ for finite $\varepsilon$. This behavior is also expected from the exact analysis in the previous section. The interesting feature both plots (Figs.4.1,4.2) have in common is the obvious phase halving in the $\varphi$-periodicity (note that $\varphi$ ranges from 0 to $1 / 2$ ). A periodicity with $\varphi / 2$ indicates, that the disorder average is dominated by correlations between time-reversed path (Cooperons in the diagrammatic language). The qualitative picture is the same for a canonical ensemble. Again $G_{\eta, \delta \delta}(\varepsilon, \varphi)$ diverges for $\eta \rightarrow 0$ and $\varepsilon>0$. On the other hand, for $\varepsilon=0$ there exists a finite limit $\lim _{\eta \rightarrow 0} G_{\eta, \delta \delta}(0, \varphi)$. This has to be the case, since in the limit $\varepsilon \rightarrow 0, \eta \rightarrow 0$ the total response function

$$
G_{\eta}(\varepsilon, \varphi):=G_{\eta, \wp}(\varepsilon, \varphi)+G_{\eta, \delta \delta}(\varepsilon, \varphi)
$$

is related to the flux derivative of the persistent current. 


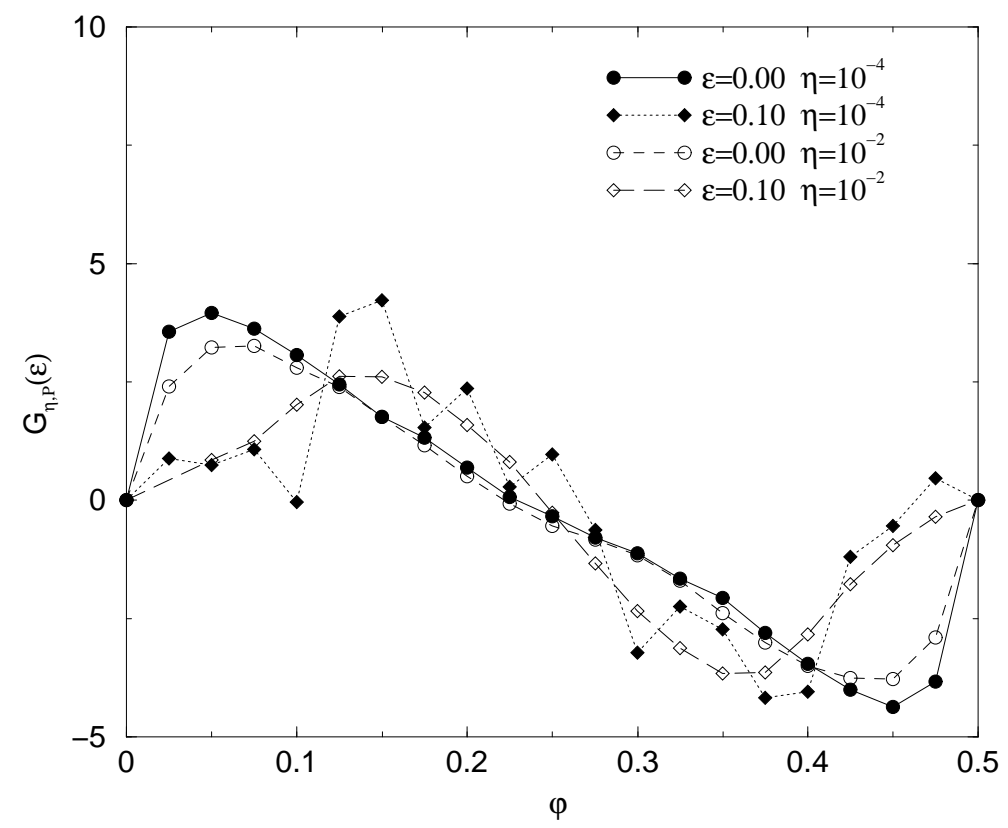

Figure 4.1: $G_{\eta, \wp}(\varepsilon, \varphi)$ for a grand canonical $10 \times 3 \times 3$ system with periodic boundary conditions, $\mu=0$ and $w=5$. It was averaged over 5.000 random disorder realizations for $\eta=10^{-2}$ and over 20.000 for $\eta=10^{-4}$.

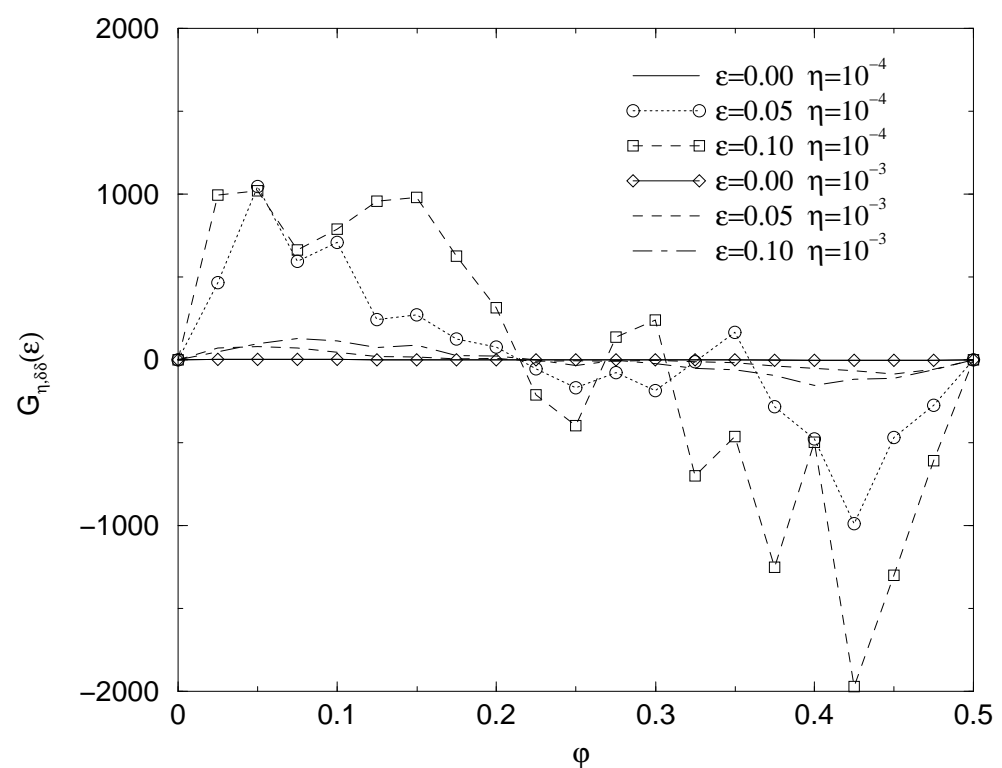

Figure 4.2: $G_{\eta, \delta \delta}(\varepsilon, \varphi)$ for the same parameters as in Fig.4.1. 


\subsubsection{The static limit of the response function}

The response to a static flux $\phi(t) \rightarrow \phi+\phi_{0} \delta \varphi$ is obtained from Eq.(4.12) by setting $\delta \varphi_{\omega}=\delta \varphi \delta(\omega)$, which yields

$$
I_{\text {static }}^{(2)}=\frac{1}{2} \frac{(-e) 8 \pi^{2}}{(m L)^{3}} K^{(2)}(0,0)(\delta \varphi)^{2} .
$$

The problem is now time independent and the question arises, how $I_{\text {static }}^{(2)}$ is related to the equilibrium persistent current $I(\varphi+\delta \varphi)$. To compare both quantities, $I(\varphi+\delta \varphi)$ is expanded in powers of $\delta \varphi$ :

$$
I(\varphi+\delta \varphi)=I(\varphi)+\left(\frac{\partial I(\varphi)}{\partial \varphi}\right) \delta \varphi+\frac{1}{2}\left(\frac{\partial^{2} I(\varphi)}{\partial \varphi^{2}}\right)(\delta \varphi)^{2}+\cdots
$$

As has been shown by Fricke and Kopietz [54], in a canonical ensemble up to second order in $\delta \varphi$ the results from the dynamic response function (in the static limit) and the equilibrium expansion are identical. This means

$$
\left(\frac{\partial^{2} I(\varphi)}{\partial \varphi^{2}}\right)_{N}=\frac{(-e) 8 \pi^{2}}{(m L)^{3}} K^{(2)}(0,0)
$$

where the index $N$ states, that the particle number is held constant. Equivalently the linear response function $K^{(1)}(\varepsilon)$ with

$$
I^{(1)}(t)=\frac{2 \pi e}{(m L)^{2}} \int_{-\infty}^{\infty} d \omega_{1} \delta \varphi_{\omega_{1}} K^{(1)}\left(\omega_{1}\right) e^{-i \omega_{1} t}
$$

is connected to the first flux-derivative of $I(\varphi)$ via

$$
\left(\frac{\partial I(\varphi)}{\partial \varphi}\right)_{N}=\frac{2 \pi^{2} e}{(m L)^{2}} K^{(1)}(0)
$$

The canonical persistent current can be calculated by an expansion in the flux and disorder dependent chemical potential $\mu(\{U\}, \varphi) \approx\langle\bar{\mu}\rangle_{\varphi}+\delta \mu[59] . I(\varphi)$ can then be expressed through the variance of the particle number fluctuation $\overline{(\delta N)^{2}}$ in a grand canonical ensemble with chemical potential $\langle\bar{\mu}\rangle_{\varphi}$ (the over-bar denotes disorder averaging and $\langle\cdots\rangle_{\varphi}$ averaging over flux $\varphi$, see [75] for a detailed derivation):

$$
I(\varphi)=\frac{(-e) \Delta}{4 \pi} \frac{\partial}{\partial \varphi} \overline{[\delta N(\varphi)]^{2}} .
$$

Altshuler and Shklovskii [64] have diagrammatically calculated the particle number fluctuations $\overline{[\delta N(\varphi, E)]^{2}}$ in an energy interval $E$ around the Fermi energy. Their 
findings have been verified numerically in Refs. [16, 75]. Presuming a constant density of states, $\overline{[\delta N(\varphi, E)]^{2}}$ becomes independent of $E$ for $E \gtrsim E_{c}$ and can be written as 75$]$

$$
\frac{\partial}{\partial \varphi} \overline{[\delta N(\varphi)]^{2}}=-\frac{8}{\pi} \sum_{j=1}^{\infty} e^{-j \sqrt{\Gamma_{0} / E_{c}}} \sin (4 \pi j \varphi)=-\frac{4}{\pi} \frac{\sin (4 \pi \varphi)}{\cosh \left(\sqrt{\Gamma_{0} / E_{c}}\right)-\cos (4 \pi \varphi)},
$$

where $\overline{[\delta N(\varphi)]^{2}}=\overline{[\delta N(\varphi, E)]^{2}}$ for $E \gtrsim E_{c} . \Gamma_{0}$ is the cutoff entering the Cooperon $C_{n i}$ which for a finite system of free electrons is given by $\Gamma_{0}=\Delta / \pi$. The canonical persistent current is completely determined by an energy interval of size $E_{c}$. From the numerical results in Ref. [75] $E_{c}$ can be identified for the tight-binding model as that value of $E$, where $\overline{[\delta N(\varphi, E)]^{2}}$ becomes constant. For the $10 \times 3 \times 3$ system with $w=5$ the Thouless energy was found to be $E_{c} \approx 1$. The cutoff $\Gamma_{0}$ might still be different from $\Delta / \pi$ in the tight-binding model and thus $\Gamma_{0} / E_{c}$ is considered as a fit-parameter. From Eqs.4.68) and(4.67) one obtains

$$
\left(\frac{\partial^{2} I}{\partial \varphi^{2}}\right)_{N}=\frac{(-e) \Delta}{4 \pi} \frac{\partial^{3}}{\partial \varphi^{3}} \overline{(\delta N)^{2}}
$$

with

$$
\frac{\partial^{3}}{\partial \varphi^{3}} \overline{(\delta N)^{2}}=64 \pi\left[3 \frac{\sin (4 \pi \varphi) \cos (4 \pi \varphi)}{[\alpha-\cos (4 \pi \varphi)]^{2}}-2 \frac{\sin ^{3}(4 \pi \varphi)}{[\alpha-\cos (4 \pi \varphi)]^{3}}+\frac{\sin (4 \pi \varphi)}{\alpha-\cos (4 \pi \varphi)}\right],
$$

where $\alpha=\cosh \left(\sqrt{\Gamma_{0} / E_{c}}\right)$. Now, inserting $\lim _{\eta \rightarrow 0} G_{\eta}(0, \varphi)=\left(2 / m^{3}\right) K^{(2)}(0,0)$ in Eq.(4.64) yields

$$
\left(\frac{\partial^{2} I(\varphi)}{\partial \varphi^{2}}\right)_{N}=\frac{(-e) 4 \pi^{2}}{L^{3}} G_{\eta}(0, \varphi) \text { with } \eta \ll \Delta
$$

and

$$
G_{\eta}(0, \varphi)=\frac{\Delta L^{3}}{2(2 \pi)^{3}} \frac{\partial^{3}}{\partial \varphi^{3}} \overline{(\delta N)^{2}} \text { with } \eta \ll \Delta,
$$

which is valid, since $G_{\eta}(0, \varphi)$ becomes independent of $\eta$ for $\eta \ll \Delta$. This relation can now be tested numerically. From fitting numerical data for $\overline{[\delta N]^{2}}$ to the theoretical prediction in Eq.(4.68) the parameter $\tilde{\Gamma}_{0}:=\Gamma_{0} / E_{c}$ was found to be $\tilde{\Gamma}_{0}=2.1[75]$. At first sight, this seems to contradict the condition $\Delta<E_{c}$ underlying the diagrammatic calculation. Since all theoretical predictions could be fit within excellent accuracy, it was argued in Ref. [75], that this discrepancy must be addressed to the difference between the two models (the tight-binding model on the numerical side, and the free electron gas model for the diagrammatic calculations) but one is indeed 


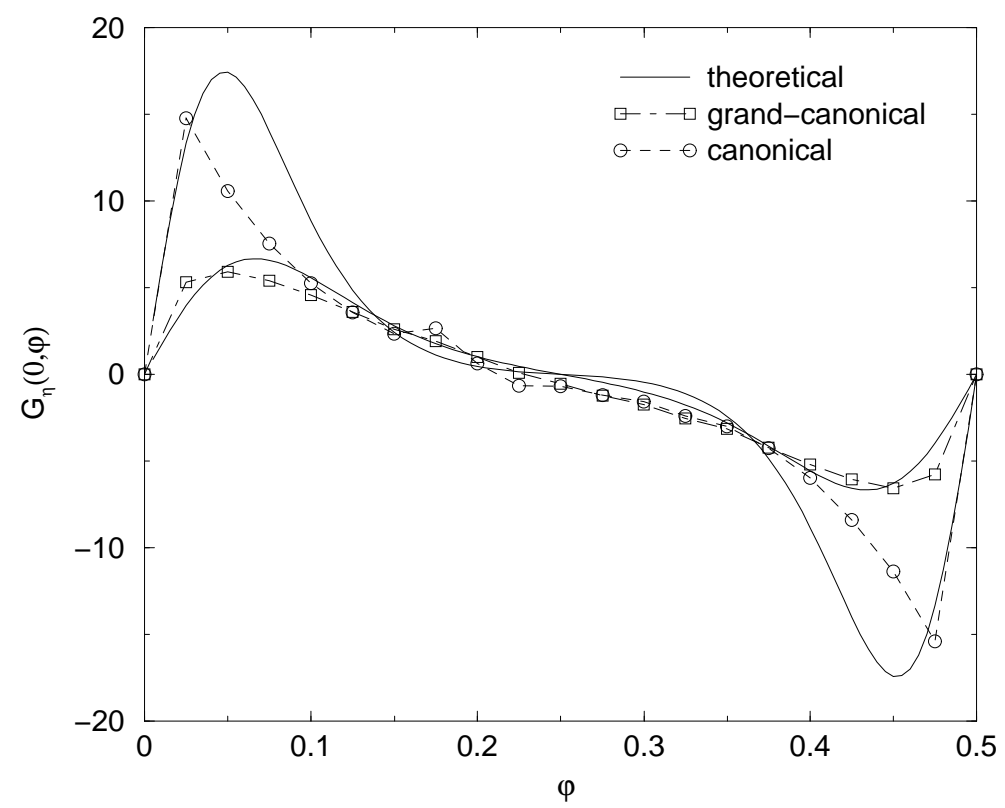

Figure 4.3: The zero frequency limit $G_{\eta}(0, \varphi)$ for the $10 \times 3 \times 3$ system with periodic boundary conditions, $w=5$ and $\eta=10^{-4}$. The theoretical curve is the AltshulerShklovskii result for $B=0.09$ and $\tilde{\Gamma}_{0}=2.1$ (canonical) or $\tilde{\Gamma}_{0}=4$ (grand canonical).

describing the same physical situation in both cases. The average density of states for the tight-binding model was numerically found to be $\Delta^{-1}=11.5$. The factor in Eq.(4.72) is thus $B:=\Delta L^{3} /\left(2(2 \pi)^{3}\right) \approx 0.18$. Figure 4.3 shows the numerical result for a canonical ensemble with fixed particle number $N=19$ (corresponding to a flux and disorder averaged chemical potential $\bar{\mu}=0$ ) and for a grand canonical one with $\mu=0$. Again only that part of the spectrum with $\left|\varepsilon_{\alpha}\right| \leq 2$ was considered. The best fit to the canonical data is obtained for $\tilde{\Gamma}=2.1$ and $B=0.09$. Thus $B$ is a factor 2 smaller than expected from the above estimate. However, as already stated, one can not expect, that all energy scales can be mapped one to one to the tight-binding model. The numerical data is therefore in good agreement with the theoretical predictions of Refs. [64, 54]. To make sure, that the apparent agreement between numerical results and theory is not just a matter of coincidence, the zero frequency limit of the linear response function $K^{(1)}(\varepsilon, \varphi)$ introduced in Eq.(4.65) was calculated as well. The explicit form of $K^{(1)}(\varepsilon, \varphi)$ is given by the sum of the diamagnetic and paramagnetic contribution $K^{(1)}(\varepsilon, \varphi)=K_{\text {para }}^{(1)}(\varepsilon, \varphi)-N(\mu, \varphi)$ with [54]

$$
K_{\text {para }}^{(1)}(\varepsilon, \varphi)=\lim _{\eta \rightarrow 0} \sum_{\alpha \beta}\left|P_{\alpha \beta}\right|^{2} \frac{f\left(\varepsilon_{\beta}\right)-f\left(\varepsilon_{\alpha}\right)}{\varepsilon_{\alpha}-\varepsilon_{\beta}-\varepsilon-i \eta} .
$$

The sum on the right hand side becomes independent of $\eta$ for $\eta \ll \Delta$ so that Eq.(4.73) can be easily evaluated numerically. From Eq.(4.66) and (4.67) it follows, 
that

$$
\frac{1}{m^{2}} K_{\text {para }}^{(1)}(0, \varphi)=-\frac{\Delta L^{2}}{2(2 \pi)^{2}} \frac{\partial^{2}}{\partial \varphi^{2}} \overline{(\delta N)^{2}}+\overline{N(\mu, \varphi)}
$$

with

$$
\frac{\partial^{2}}{\partial \varphi^{2}} \overline{(\delta N)^{2}}=-16\left[\frac{\cos (2 \pi \varphi)}{\cosh \left(\sqrt{\tilde{\Gamma}_{0}}\right)-\cos (4 \pi \varphi)}-\frac{\sin ^{2}(4 \pi \varphi)}{\left[\cosh \left(\sqrt{\tilde{\Gamma}_{0}}\right)-\cos (4 \pi \varphi)\right]^{2}}\right] .
$$

Comparing the prefactor $A:=-\Delta L^{2} /\left[2(2 \pi)^{2}\right]$ with $B:=\Delta L^{3} /\left(2(2 \pi)^{3}\right)$ yields $A=-(2 \pi / L) B$. From $B=0.09$ and $L=10$ one would thus expect $A=-0.057$. According to Eqs.(4.74), (4.75) the flux average of $K^{(1)}(0, \varphi)$ vanishes and thus the flux independent part of $K_{\text {para }}^{(1)}(\varepsilon, \varphi)$ should be canceled by the diamagnetic term $-N(\mu, \varphi)$. Numerically an exact cancellation is hard to observe since the particle number sensitively depends on the density of states, which in the computer simulations is only approximately constant (and not fixed like it is assumed in the analytical calculations). The flux dependence of the average grand canonical particle number was found to be negligible compared to that of $K_{\text {para }}^{(1)}(0, \varphi)$. In order to compare the obtained data with the theoretical prediction, $\overline{N(\mu, \varphi)}$ in Eq. (4.74) is replaced by the (numerical) flux-average of $\bar{N}_{a v}=\overline{\left\langle K_{\text {para }}^{(1)}(0, \varphi)\right\rangle_{\varphi}}$. Overall the numerical paramagnetic linear response function for the $10 \times 3 \times 3$ system with $w=5$ is expected to be given by

$$
\frac{1}{m^{2}} K_{\text {para }}^{(1)}(0, \varphi)=-0.057 \frac{\partial^{2}}{\partial \varphi^{2}} \overline{(\delta N)^{2}}+\bar{N}_{a v} .
$$

Fig.4.4 shows a comparison between the actual numerical data for $\frac{1}{m^{2}} K_{\text {para }}^{(1)}(0, \varphi)$ and the prediction from Eq.(4.76). The values for the parameter $\tilde{\Gamma}_{0}$ are those obtained from the fit to the quadratic response function $K^{(2)}$ (see Fig.4.3). Recalling that all fit parameters, except for the flux independent shift $\bar{N}_{a v}$, were determined a priori, the obtained agreement is convincing. As expected, in a canonical ensemble the static limit of the first and second order response function agrees with the corresponding flux-derivative of the persistent current, which is again well described by the Altshuler-Shklovskii result. The $10 \times 3 \times 3$ system with $w=5$ seem therefore well suited to study diffusive metallic Aharonov-Bohm rings. As was already noted, the response functions are formally identical for a grand canonical and canonical ensemble if in the latter case the chemical potential $\mu(U, \varphi)$ is interpreted as a function of disorder and flux. In contrast, the flux derivative of the persistent current differs in the two cases by terms containing the derivative of the occupation function $f$ (which vanish in the canonical case) [54]. The grand canonical persistent current in the diffusive regime is known to be exponentially small [65], while the canonical one is described by Eqs.(4.67) and (4.68). Thus the zero frequency limit of the response function at fixed $\mu$ can not be identified with derivatives of the 


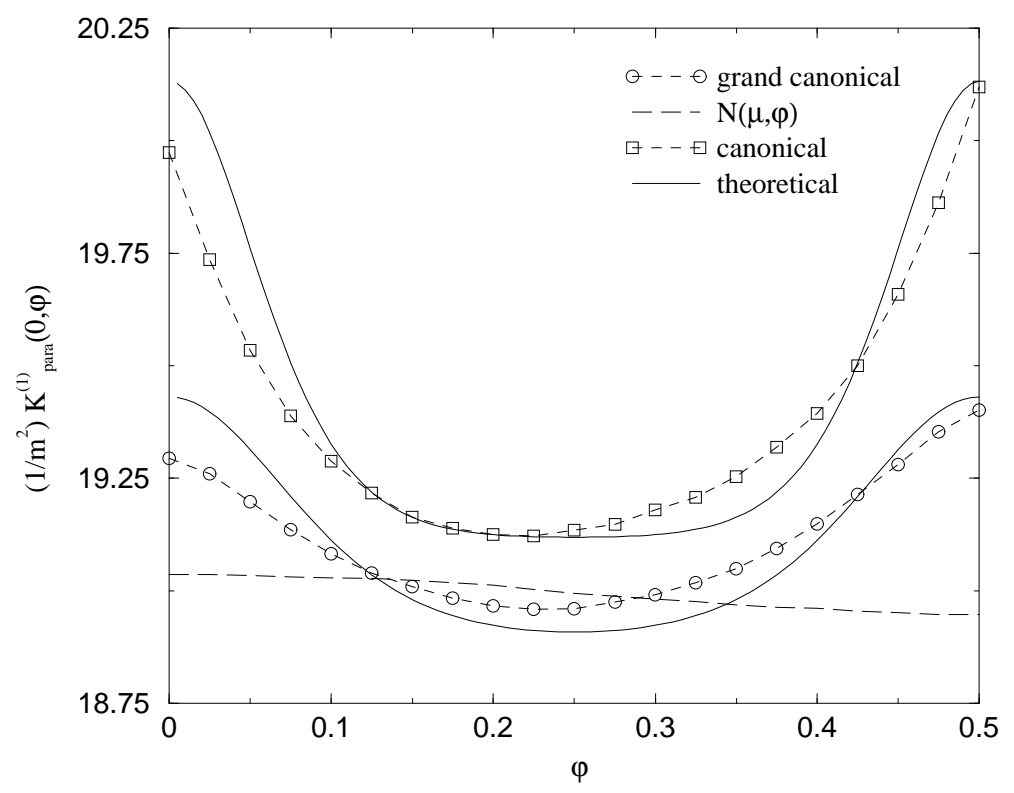

Figure 4.4: The zero frequency limit $\left(1 / \mathrm{m}^{2}\right) K_{\text {para }}^{(1)}(0, \varphi)$ of the paramagnetic linear response function for the $10 \times 3 \times 3$ system with periodic boundary conditions, $w=5$ and $\eta=10^{-4}$. The theoretical curve is the prediction from Eq.(4.76) with $\tilde{\Gamma}_{0}=2.1$ (canonical) or $\tilde{\Gamma}_{0}=4$ (grand canonical). The long-dashed line is the average grand canonical particle number $\overline{N(\mu, \varphi)}$.

grand canonical thermodynamic equilibrium current. Analytically it is still hard to estimate the effect of a fluctuating chemical potential $\mu(U, \varphi)$ compared to a fixed on. Therefore the response function was studied numerically for both cases. It is interesting to notice, that no qualitative difference between the canonical and grand canonical ensemble is found. Numerically the response functions for the two ensembles differ significantly only at the edges of the plotted interval. Indeed, the grand canonical curve can be fit with the same value for the prefactors $A$ and $B$ but a different parameter $\tilde{\Gamma}_{0}$, namely $\tilde{\Gamma}_{0}=4$. This indicates, that only the effective ratio $\Gamma_{0} / E_{c}$ changes with the ensemble. The average density of states at the Fermi energy $\Delta$ and the size $L$ are independent of the statistical ensemble and therefore $A$ and $B$ remain unaffected.

\subsubsection{The response function for finite frequencies $\varepsilon>0$}

Next the case $\varepsilon>0$ is investigated. Before calculating the time-independent contribution $I_{t h}^{(2)}$ numerically, it is instructive to look at the canonical persistent current defined in Eq.(4.67). From the numerical estimate $B=0.09=:\left(\Delta_{e f f} L^{3}\right) /\left(2(2 \pi)^{3}\right)$ it follows, that the effective level spacing is approximately half the tight-binding level 
spacing $\Delta_{\text {eff }} \approx \Delta / 2 \approx 1 / 23$. Replacing $\Delta$ in Eq.(4.67) by $\Delta_{\text {eff }}$ and using Eq.(4.68) yields a persistent current for the $10 \times 3 \times 3$ system of order

$$
\frac{I(\varphi)}{(-e)}=-\frac{\Delta_{e f f}}{\pi^{2}} \frac{\sin (4 \pi \varphi)}{\cosh \left(\sqrt{\tilde{\Gamma}_{0}}\right)-\cos (4 \pi \varphi)} \approx-4.4 \times 10^{-3} \frac{\sin (4 \pi \varphi)}{\cosh (\sqrt{2.1})-\cos (4 \pi \varphi)},
$$

where again $\tilde{\Gamma}_{0}=2.1$ was used. This function is plotted in Fig. 4.5 . $I(\varphi) /(-e)$ is negative for small $\varphi$ and thus the current is paramagnetic in the zero field limit. Experimentally the current was found to be diamagnetic [55] for a relatively small ensemble of only 30 rings. It was argued, that this number might be too small to yield a properly averaged current [66] so that this point is still controversial. In the original experiment by Levy et al. [50] on $10^{7}$ copper rings the sign of the current was not measured. On the other hand, the time-independent non-linear current $I_{0}^{(2)}$

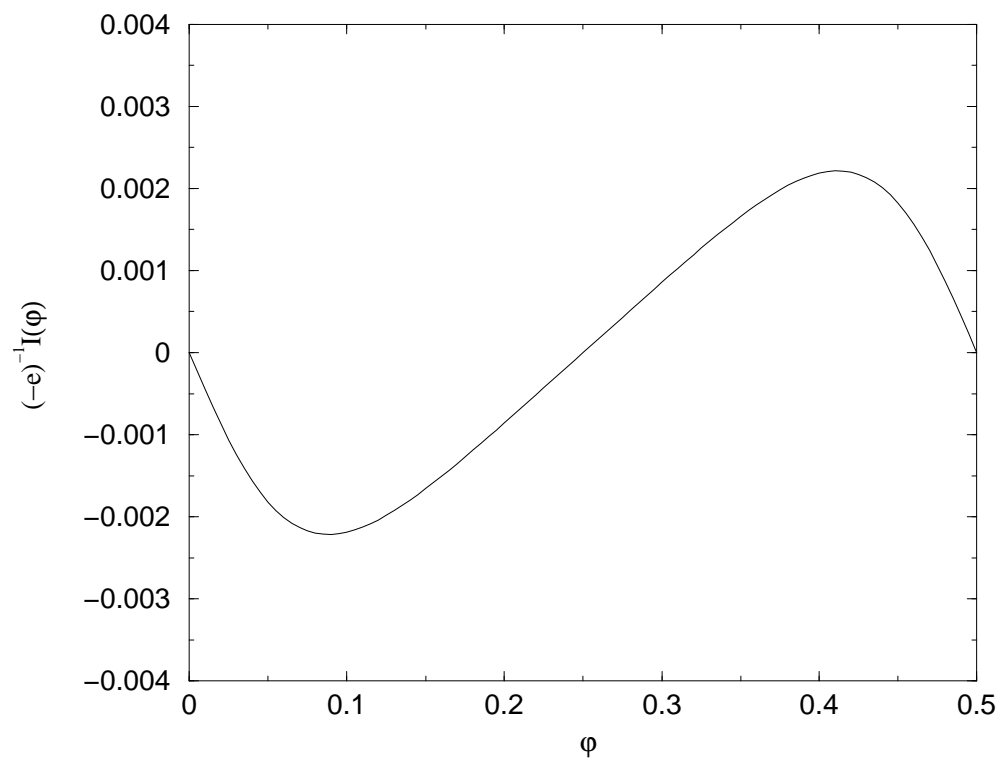

Figure 4.5: Canonical persistent current I in units of the electron charge - $e$ for the $10 \times 3 \times 3$ system as derived from Eq.(4.77).

calculated by $\mathrm{KY}$ is diamagnetic (for spinless electrons, in the presence of spin-orbit scattering the sign might reverse [66]) and of the same order of magnitude as the experimentally measured current for an ensemble of rings [56, 66]. It has therefore recently been argued, that the observed current is not an equilibrium quantity but rather a noise induced non-linear effect [66]. Now, what happens in the discrete spectrum limit? In order to compare the numerical result with that obtained by KY, a grand canonical ensemble with periodic boundary conditions in $y$ and $z$ direction is used. Fig.4.6 shows $G_{\eta, \wp}(\varepsilon, \varphi)$ as a function of $\varphi$ for different frequencies $\varepsilon$. It 


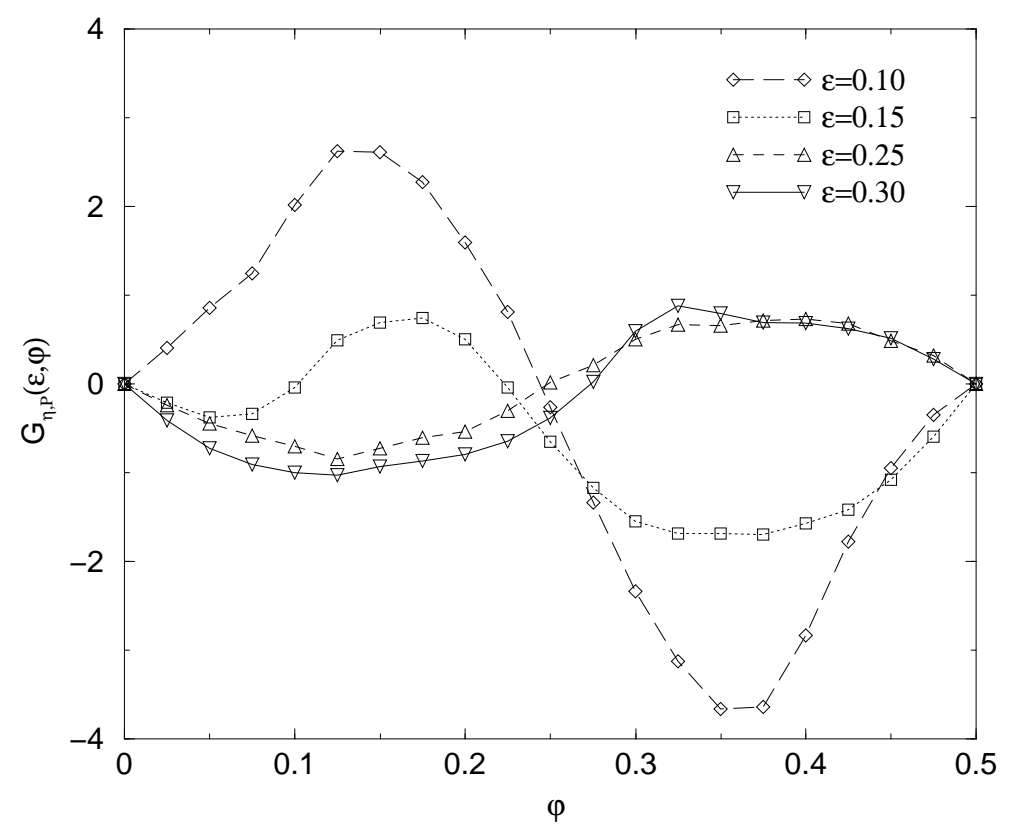

Figure 4.6: $G_{\eta, \wp}(\varepsilon, \varphi)$ for a grand canonical $10 \times 3 \times 3$ system with $\mu=0, w=5$ and $\eta=10^{-2}$, periodic boundary conditions.

was assumed in the introduction to this chapter, that for frequencies $\varepsilon<E_{c}$ the response function should be completely determined by an energy interval of order $E_{c}$, centered around the Fermi energy. In the zero frequency limit this is obviously the case due to the relation to the flux-derivatives of the persistent current. For $\varepsilon>0$ this assumption can be verified numerically. Therefore the calculations were redone with the energy spectrum restricted to $\left|\varepsilon_{\alpha}\right|<E_{c} \approx 1$. The result is shown in Fig.4.7. Compared to that for $\left|\varepsilon_{\alpha}\right|<2$ in Fig.4.6, there is, as expected, no significant dependence on the size of the energy interval. In both cases $G_{\eta, \wp}(\varepsilon, \varphi)$ shows a similar behavior. The most interesting observation is, that the function changes qualitatively as soon as $\varepsilon \gtrsim \Delta \approx 0.09$. The strong anharmonicity in the flux-dependence vanishes in this frequency regime. A further increase of $\varepsilon$ eventually changes the sign of $G_{\eta, \wp}(\varepsilon, \varphi)$. From $I_{\eta t, \wp}^{(2)}=A_{\varepsilon} m^{3} e^{\eta t} G_{\eta, \wp}(\varepsilon, \varphi)$ it follows that

$$
\frac{I_{t h}^{(2)}}{(-e)}=\lim _{\eta \rightarrow 0} \frac{I_{\eta t, \wp}^{(2)}}{(-e)}=\frac{\pi^{2}}{L^{3}}\left(\frac{\phi_{\varepsilon}}{\phi_{0}}\right)^{2} G_{\tilde{\eta}, \wp}(\varepsilon, \varphi) \text { with } \tilde{\eta} \ll \Delta .
$$

The function $G_{\eta, \wp}(\varepsilon, \varphi)$ becomes independent of $\eta$ for $\eta \ll \Delta$ and thus the limit $\lim _{\eta \rightarrow 0} G_{\eta, \wp}(\varepsilon, \varphi)$ can be replaced by $G_{\tilde{\eta}, \wp}(\varepsilon, \varphi)$ with an arbitrary $\tilde{\eta} \ll \Delta$. For $\varepsilon>\Delta$ the current $I_{t h}^{(2)}$ thus has the same sign as the persistent current $I(\varphi)$. With

\footnotetext{
${ }^{3}$ For reminder: $E_{c} \approx 1$ is the numerically estimated value from comparison with results for $\overline{[\delta N(\varphi, E)]^{2}}$.
} 


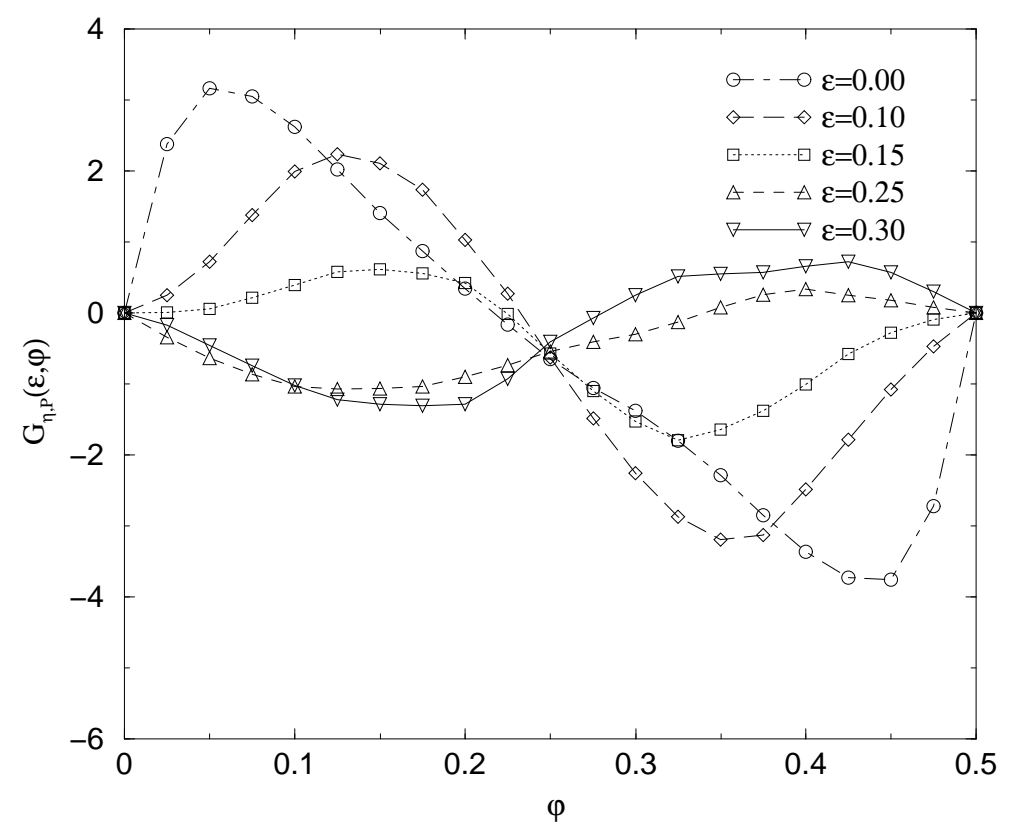

Figure 4.7: $G_{\eta, \wp}(\varepsilon, \varphi)$ for the same parameters as in Fig. 4.6, but the energy spectrum restricted to $\left|\varepsilon_{\alpha}\right|<1 \approx E_{c}$. All other plots were obtained for $\left|\varepsilon_{\alpha}\right|<2$.

$L=10$ one gets $\pi^{2} / L^{3} \approx 1 / 100$. Using $\phi_{\varepsilon} / \phi_{0} \approx 1$ as an upper bound for $\phi_{\varepsilon}$ one obtains a current $I_{t h}^{(2)}$ that is maximally one order of magnitude lager than $I(\varphi)$ in Fig.4.5. Obviously $I_{\eta t, \wp}^{(2)}$ does not yield a diamagnetic current for $\varepsilon>\Delta$. The question arising is, whether such a contribution can be identified within the numerical simulations. The calculation by KY is based on the assumption of a continuous spectrum. Formally $\eta$ enters the denominator of the response function like the imaginary part of the single particle energies $\varepsilon_{\alpha}$ (see Eq.(4.28)). From this point of view $\eta$ may be interpreted as an inelastic level broadening and the spectrum is effectively continuous for $\eta \gtrsim \Delta$. According to $\mathrm{KY}$ the time independent second order contribution to the current is given by [56]

$$
\frac{I_{0, K Y}^{(2)}}{(-e)}=8 \pi E_{c}\left(\frac{\phi_{\varepsilon}}{\phi_{0}}\right)^{2} \frac{\sin (4 \pi \varphi)}{\cosh \left(\sqrt{\tilde{\Gamma}_{0}}\right)-\cos (4 \pi \varphi)} .
$$

The flux dependence is thus the same as for the persistent current (see Eq.(4.77)) but the sign is opposite. Now if $\eta$ is viewed as some intrinsic level broadening, the factor $e^{2 \eta t}$ from the switching on procedure may be dropped and one defines the time independent current $I_{0, \eta}^{(2)}:=A_{\varepsilon} m^{3} G_{\eta}(\varepsilon, \varphi)$ which can be compared with $I_{0, K Y}^{(2)}$. The function $G_{\eta}(\varepsilon, \varphi)$ defined in Eq. (4.61) is evaluated numerically and is compared 
with the KY prediction

$$
G_{\eta}^{K Y}(\varepsilon, \varphi)=\frac{8 L^{3} E_{c}^{K Y}}{\pi} \frac{\sin (4 \pi \varphi)}{\cosh \left(\sqrt{\tilde{\Gamma}_{0}}\right)-\cos (4 \pi \varphi)} .
$$

which is defined via $I_{0, K Y}^{(2)}:=A_{\varepsilon} m^{3} G_{\eta}^{K Y}(\varepsilon, \varphi)$. Here the Thouless energy $E_{c}$ has been replaced by $E_{c}^{K Y}$ which is considered as a numerical fit parameter. The value for $\tilde{\Gamma}_{0}$ has already been fixed for the zero-frequency limit (here $\tilde{\Gamma}_{0}=4$ ). Fig.4.8 shows $G_{\eta}(\varepsilon, \varphi)$ for $\eta=0.1 \gtrsim \Delta$ and different values of $\varepsilon$. In the considered frequency interval the function varies indeed only weakly with $\varepsilon$ and the sign and flux dependence are in agreement with the KY result. The order of magnitude can not be confirmed. In order to fit $G_{\eta}(\varepsilon, \varphi)$ to $G_{\eta}^{K Y}(\varepsilon, \varphi)$, one has to choose $E_{c}^{K Y} \approx 10^{-3}$ in Eq.(4.80) which is two orders of magnitude smaller than $\Delta$ and even three orders smaller than the previous estimate $E_{c} \approx 1$. Such a huge discrepancy can hardly be addressed to numerical factors arising in the tight-binding model. Replacing $E_{c}$ by $E_{c}^{K Y} \approx 10^{-3}$ in Eq.(4.79) and comparing the corresponding current with the persistent current in Eq.(4.77) it is seen, that both have the same order of magnitude. (Again $\phi_{\varepsilon} / \phi_{0} \approx 1$ is used as an upper bound.) $G_{\eta}$ was also numerically investigated for $\eta<\Delta$. It was found, that for frequencies $0.4 \lesssim \varepsilon \lesssim 0.7$ the sign and flux dependence of the total non-linear response function $G_{\eta}$ can still be described by the KY result. However, $G_{\eta, \delta \delta}$ and therewith the amplitude of the current $I_{0, \eta}^{(2)}$ grows like $1 / \eta$. As pointed out in the previous section, this is the result of an incorrect limiting procedure. For $\eta \gtrsim \Delta$ where the spectrum can be considered as effectively continuous, the numerically calculated current $I_{0, \eta}^{(2)}$ is about three orders of magnitude smaller than the KY prediction $I_{0, K Y}^{(2)}$ if one uses $E_{c} \approx 1$ as an realistic estimate for the Thouless energy in the $10 \times 3 \times 3$ system.

So far the finite frequency response was studied for a grand canonical ensemble with periodic boundary conditions. KY implicitly assume that the response function does not depend on the statistical ensemble since their calculations where performed at fixed $\mu$ while in experiments $N$ is kept constant on each ring. In the zero frequency limit $\varepsilon \rightarrow 0$ both cases have already been compared in Fig.4.3 and no qualitative difference was found. The corresponding plot for $\varepsilon>0$ is shown in Fig.4.9. The difference between the grand canonical and canonical curves is marginal. For finite frequencies the non linear response function $G_{\eta, \wp}(\varepsilon, \varphi)$ seems to be completely insensitive to the underlying statistical ensemble.

\subsubsection{Influence of the boundary conditions in transverse di- rections}

There is yet another effect to be studied: In experiments not only the particle number on each ring is constant but also the electrons motion in the transverse direction 


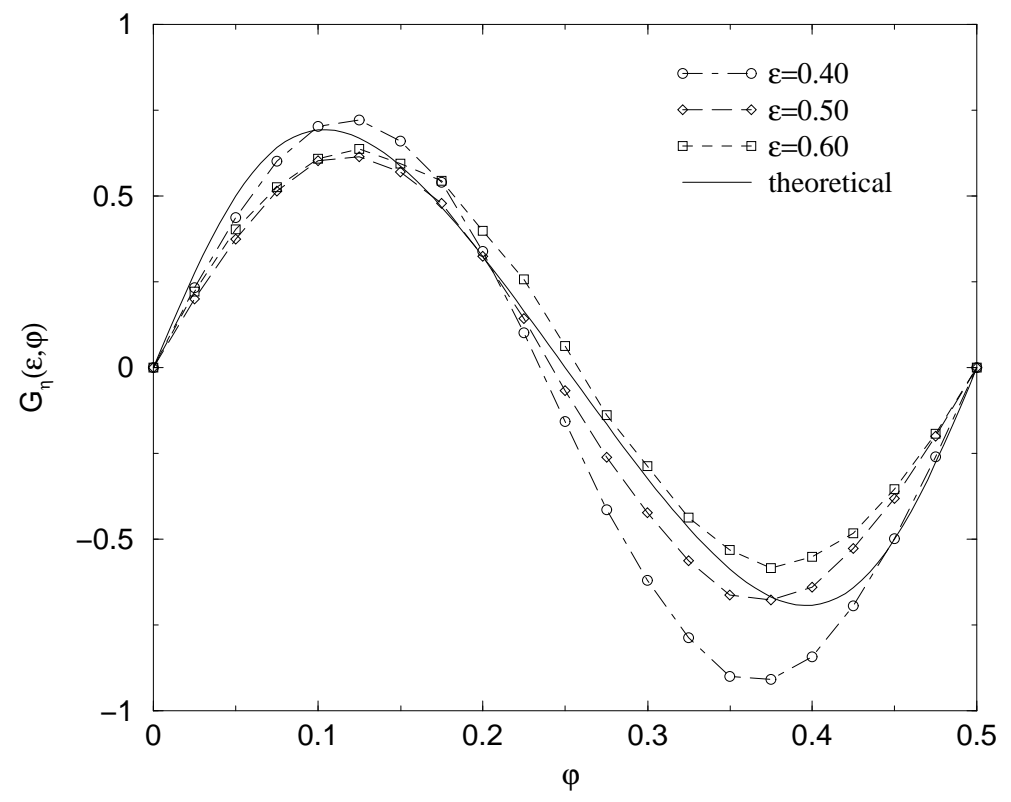

Figure 4.8: $G_{\eta}(\varepsilon, \varphi)$ for $\eta=0.1$. The theoretical curve is the Kravtsov-Yudson result with $\tilde{\Gamma}_{0}=4.0$ and $E_{c}^{K Y}=10^{-3} \approx 10^{-2} \Delta \approx 10^{-3} E_{c}$. Again the estimate $E_{c} \approx 1$ is used.

is bounded by the surface of the ring. For the theoretical calculations periodic boundary conditions in $y$ and $z$ direction are used and it is implicitly assumed, that the response function is insensitive to the choice of boundary conditions as well. In a large enough sample with $1 / k_{F} \ll a \sim l$ (where $a$ is the thickness of the ring) this seems to be a reasonable assumption. A numerical justification for the equilibrium persistent current problem has been given in Ref. [75]. It was found, that the current, numerically calculated form Eq.(4.67) with fixed boundary conditions, is in excellent agreement with the theoretical prediction by Altshuler and Shklovskii who used periodic conditions. The computational effort to calculate $G_{\eta, \wp}$ is unfortunately much higher than for $\overline{(\delta N)^{2}}$ in the persistent current problem. To obtain the matrix elements $P_{\alpha \beta \gamma}$ not only the eigenvalues but also the eigenvectors are needed. This for itself drastically increases the necessary computing time and the summation over the three variables $\alpha, \beta, \gamma$ makes things even worse. The computer program's run-time increases rapidly with growing system size. Already the time needed by the Lanczos procedure to calculate the eigenvalues and eigenvectors grows $\sim N_{\text {tot }}^{2}$ and on top of that the decreasing level spacing together with the condition $\eta \ll \Delta$ strongly increases the number of averagings which are necessary to obtain smooth curves. Most of the simulations where therefore done on the relatively small $10 \times 3 \times 3$ system. In such a small sample with only 3 lattice sites in $y$ and $z$ direction a change in the boundary conditions still leads to relatively large energy 


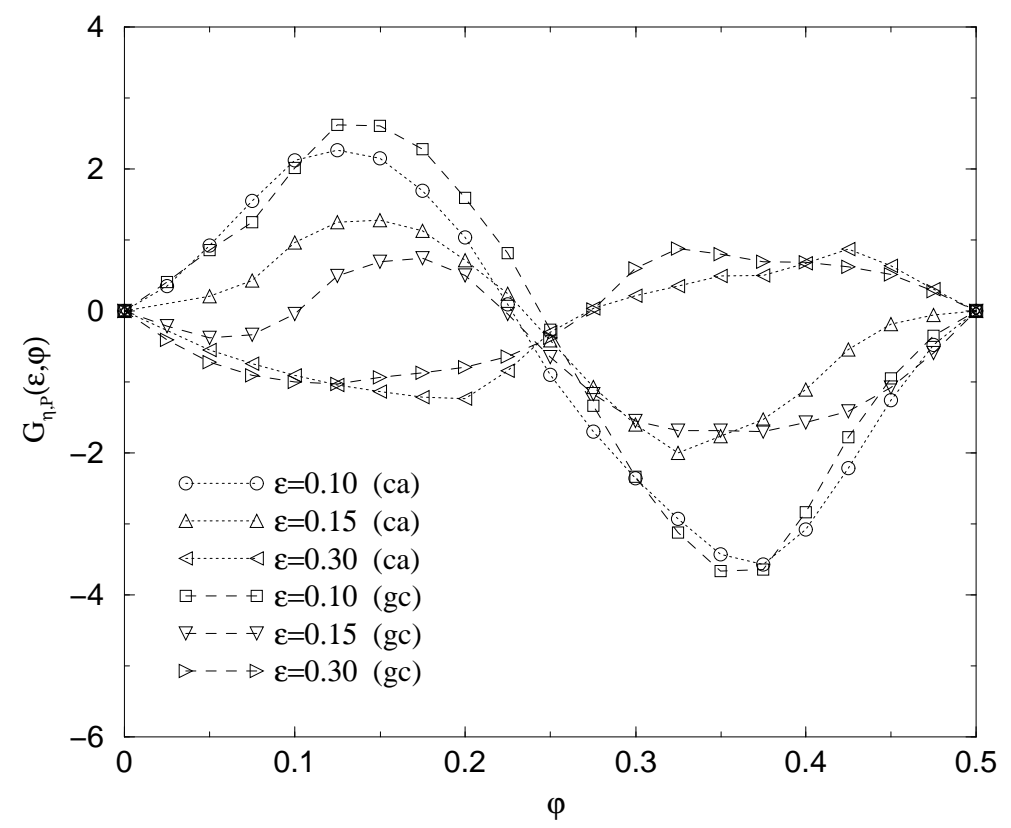

Figure 4.9: Comparison of $G_{\eta, \wp}(\varepsilon, \varphi)$ for the canonical (ca) and grand canonical (gc) $10 \times 3 \times 3$ ensemble with periodic boundary conditions, $w=5$ and $\eta=10^{-2}$. Dotted lines are the results for fixed particle number $N=19$, corresponding to $\bar{\mu}=0$. Dashed lines where obtained for fixed chemical potential $\mu=0$.

shifts. In the canonical ensemble with $N=19$ for example, changing from periodic to fixed boundaries shifts the flux- and disorder average of the chemical potential from $\bar{\mu}_{\text {peri }}=0.0$ to $\bar{\mu}_{\text {fix }}=-0.3$. Comparing this energy scale with the strong $\varepsilon$ dependence on a similar scale shown in Fig.4.6, one can not expect the system to be completely insensitive to its boundaries. Fig.4.10 shows $G_{\eta, \wp}(\varepsilon, \varphi)$ calculated for fixed boundary conditions. In order to compare the canonical with the grand canonical result, in the latter case the chemical potential was shifted to $\mu=-0.3$ as well. For small frequencies $\varepsilon \leq \Delta$ the results are in qualitative agreement with those in Figs.4.3 and 4.9. Also the change of sign is observed for $\varepsilon>\Delta$ though the curve for $\varepsilon=0.3$ shows no phase halving. This is addressed to the strong $\varepsilon$ dependence in the plotted region and the relatively large change in the chemical potential. The function $G_{\eta, \delta \delta}$ with finite, fixed $\eta$ shows a much weaker $\varepsilon$ dependence and one can therefore expect it to be less sensitive to boundary conditions. Fig.4.11 shows the corresponding data for the grand canonical ensemble with average particle number $\bar{N}=19$. Indeed, it is seen that the qualitative behavior of $G_{\eta, \delta \delta}$ does not change in terms of the boundaries. Only the amplitude of the oscillations is moderately rescaled. The more important insight from Fig.4.10 is, that the response function is again independent of the statistical ensemble. The only notable difference occurs at $\varepsilon=0$ for $\varphi$ in the vicinity of 0 or $1 / 2$ respectively. As in Fig.4.3 this difference is 


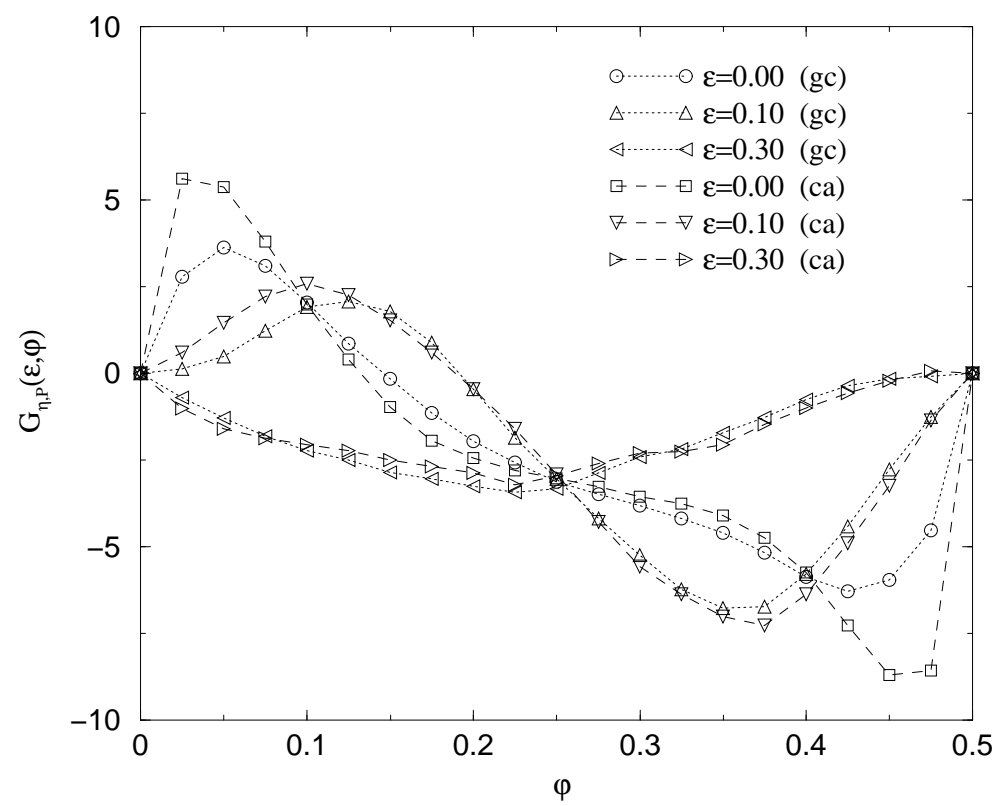

Figure 4.10: Comparison between $G_{\eta, \wp}(\varepsilon, \varphi)$ for the canonical (ca) and grand canonical (gc) $10 \times 3 \times 3$ ensemble with fixed boundary conditions, $w=5$ and $\eta=10^{-2}$. Dotted lines are the results for fixed particle number $N=19$, corresponding to $\bar{\mu}=-0.3$. Dashed lines where obtained for fixed chemical potential $\mu=-0.3$.

only quantitative and may be explained by a different effective parameter $\tilde{\Gamma}_{0}$.

\subsubsection{The linear in time contribution to the current}

Next the linear in time contribution $d I_{k i n}^{(2)} / d t$ is investigated. For numerical calculations it is rewritten as $d I_{k i n}^{(2)} / d t=: A_{\varepsilon} m^{3} \lim _{\eta \rightarrow 0} G_{\eta, k i n}(\varepsilon, \varphi)$ with

$$
G_{\eta, k i n}(\varepsilon, \varphi)=-2 \eta \sum_{\alpha \beta} \frac{P_{\alpha \beta \alpha}}{m^{3}}\left[\frac{f\left(\varepsilon_{\alpha}\right)-f\left(\varepsilon_{\beta}\right)}{\left(\varepsilon_{\alpha}-\varepsilon_{\beta}-\varepsilon\right)^{2}+\eta^{2}}+\frac{f\left(\varepsilon_{\alpha}\right)-f\left(\varepsilon_{\beta}\right)}{\left(\varepsilon_{\alpha}-\varepsilon_{\beta}+\varepsilon\right)^{2}+\eta^{2}}\right],
$$

and in the limit $T \rightarrow 0$ one gets

$$
\begin{aligned}
G_{\eta, k i n}(\varepsilon, \varphi)=-2 \sum_{\varepsilon_{\alpha} \leq \mu} \sum_{\varepsilon_{\beta}>\mu} \frac{\left|P_{\alpha \beta}\right|^{2}}{m^{2}}\left(\frac{P_{\alpha \alpha}}{m}-\frac{P_{\beta \beta}}{m}\right) & \left(\frac{\eta}{\left(\varepsilon_{\alpha}-\varepsilon_{\beta}+\varepsilon\right)^{2}+\eta^{2}}\right. \\
& \left.+\frac{\eta}{\left(\varepsilon_{\alpha}-\varepsilon_{\beta}-\varepsilon\right)^{2}+\eta^{2}}\right)
\end{aligned}
$$




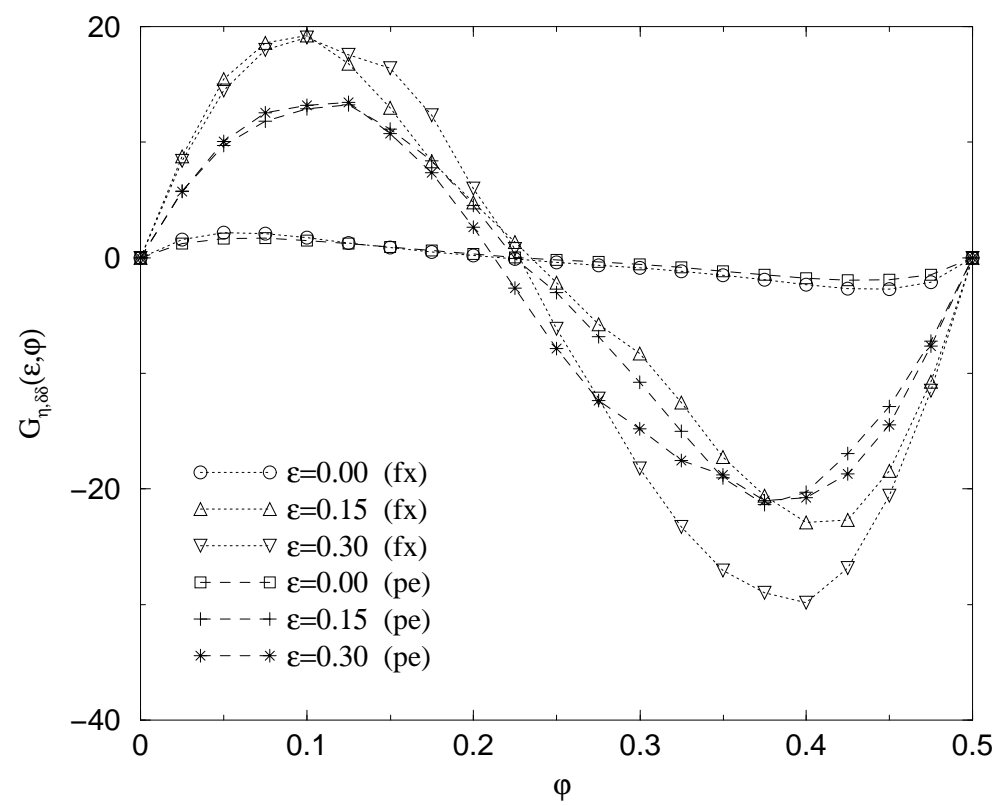

Figure 4.11: Comparison between $G_{\eta, \delta \delta}(\varepsilon, \varphi)$ for fixed ( $\left.f x\right)$ and periodic (pe) boundary conditions. (Grand canonical $10 \times 3 \times 3$ system with $w=5, \eta=10^{-2}, \bar{N}=19$ ). Dotted lines are the results for ( $f x)$ with $\mu=-0.3$, dashed lines for (pe) with $\mu=0$.

As expected it is found numerically, that $G_{\eta, k i n}(\varepsilon, \varphi)$ becomes independent of $\eta$ for $\eta \ll \Delta$. The current $I_{k i n}^{(2)}$ is thus related to $G_{\eta, k i n}(\varepsilon, \varphi)$ via

$$
\frac{d I_{\text {kin }}^{(2)}}{d t}=\frac{(-e) \pi^{2}}{L^{3}}\left(\frac{\phi_{\varepsilon}}{\phi_{0}}\right)^{2} G_{\eta, k i n}(\varepsilon, \varphi) \text { with } \eta \ll \Delta .
$$

Keeping in mind, that the golden rule result is only valid for times $t<1 / \Delta$, one can estimate the maximal contribution $I_{k i n, \max }^{(2)}$ to the total current as

$$
\frac{I_{k i n, \max }^{(2)}}{(-e)} \approx \frac{\pi^{2}}{\Delta L^{3}} G_{\eta, k i n}(\varepsilon, \varphi) .
$$

Fig.4.12 shows numerical results for $G_{\eta, k i n}(\varepsilon, \varphi)$ in a grand canonical ensemble. Obviously $G_{\eta, k i n}(\varepsilon, \varphi)$ gives rise to a diamagnetic current, i.e. the sign is opposite to that of the persistent current (see Fig.4.5). For the $10 \times 3 \times 3$ system the factor in Eq.(4.84) is given by $\pi^{2} /\left(\Delta L^{3}\right) \approx 1 / 10$. Comparing Fig.4.12 to $I(\varphi)$ in Fig.4.5 one thus finds a maximal linear in time current $I_{k i n, \max }^{(2)}$ that is about two orders of magnitude lager than the corresponding canonical persistent current. The data for the canonical ensemble is shown in Fig.4.13. Obviously the result is again not sensitive to the underlying statistical ensemble. The flux periodicity with period $1 / 2$ indicates, that $G_{\eta, k i n}(\varepsilon, \varphi)$ is governed by the diffusive dynamics of the electrons with strong correlations between time-reversed trajectories. 


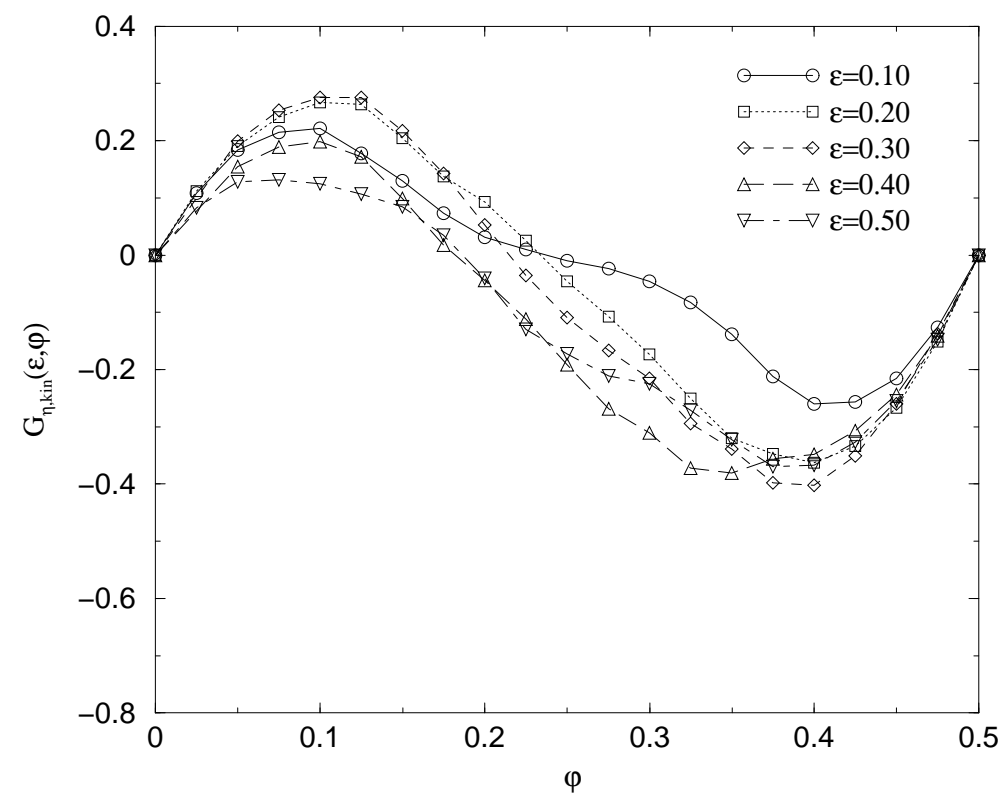

Figure 4.12: $G_{\eta, k i n}(\varepsilon, \varphi)$ for a grand canonical $10 \times 3 \times 3$ system with periodic boundary conditions, $\mu=0, w=5$ and $\eta=10^{-2}$.

\subsection{Conclusions}

In this chapter it was demonstrated, that the calculation of the second order response function contains some subtleties that are overlooked in the usual diagrammatic Green's function approach. An exact treatment shows the existence of a contribution $I_{k i n}^{(2)}(t)$ to the current, that increases linearly with time. From the well-known derivation of Fermi's golden rule [63] it is clear that this result is only valid in an intermediate time interval which requires $t<1 / \Delta$. In particular, the calculation of the long-time behavior of the non-equilibrium current requires non-perturbative methods. For a continuous spectrum infinite orders in the external field can be treated by solving the partial differential equation for the Cooperon [57]. In the discrete spectrum limit the inclusion of higher order terms remains an open problem. One should keep in mind that the calculation presented here has been performed for non-interacting electrons in a random potential, so that the results are valid as long as the spectrum of the system is discrete in the sense that the single-particle level broadening $\gamma_{q p} \approx 1 / \tau_{e e}$ (for low temperatures) is smaller than $\Delta$. In the zero temperature limit $1 / \tau_{e e}$ depends only on the quasiparticle energy $\xi_{\alpha}$. It was shown that for external frequencies $\varepsilon<E_{c}$ the response function is then completely determined by the discrete part of the spectrum. However, as soon as the spectrum has a discrete part, the Green's function approach is not controlled anymore, since the low energy single particle excitations can not be described correctly. Of course experiments 


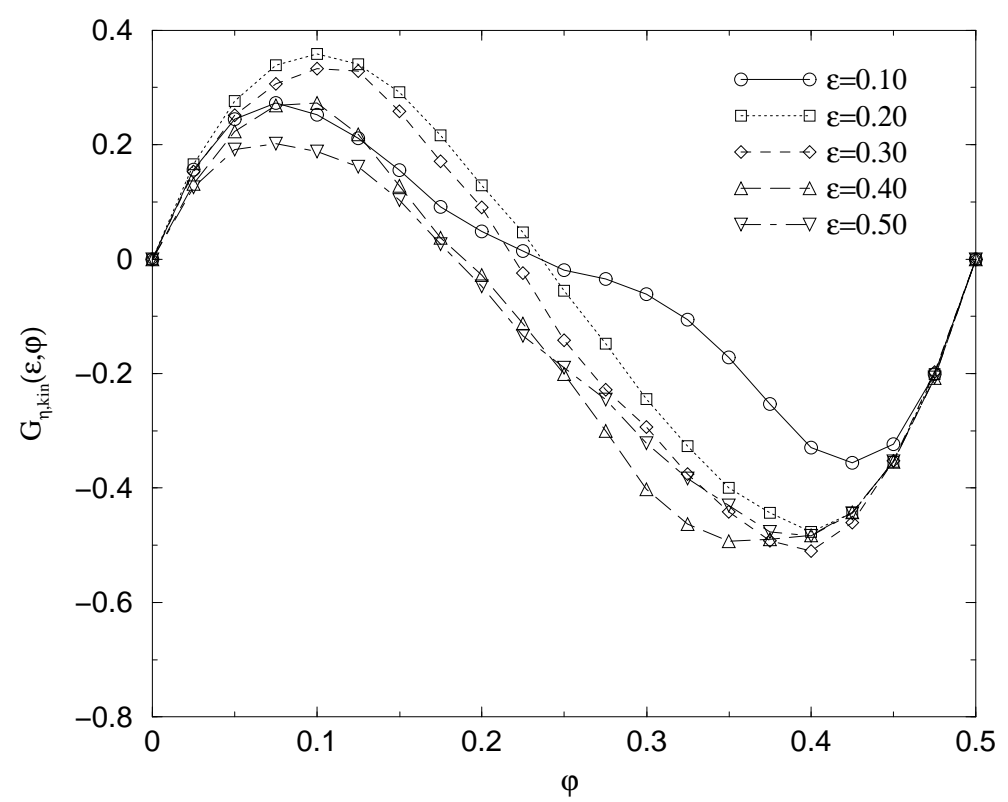

Figure 4.13: $G_{\eta, k i n}(\varepsilon, \varphi)$ for a canonical $10 \times 3 \times 3$ system with periodic boundary conditions, $N=19, \bar{\mu}=0, w=5$ and $\eta=10^{-2}$.

are not performed at zero temperature and all levels will have a finite broadening. To estimate the temperature, where the spectrum becomes discrete, one can use the (continuous-spectrum-) result [20] $1 / \tau_{e e}(T)=\Delta\left(T / E_{c}\right)^{d / 2}$. Thus the spectrum will become discrete for $T<E_{c}$. The experiment by Mohanty et al. [55] was performed on gold rings with diameter $2.6 \mu \mathrm{m}$ and Thouless energy $E_{c} \approx 7.3 \mathrm{mK}$ down to temperatures $T=5.5 \mathrm{mK}$. Thus even for such relatively large rings the discrete spectrum limit may be reached. For smaller samples of size nano-meters [48] one can not expect a continuous spectrum any more.

It is important to point out that the linear time-dependence of the current is a consequence of the discrete spectrum, and is not related to the adiabatic switching on procedure in Eq.(4.28). Sudden switching on yields the same linear time-dependence of the current.

In Ref. [55] the average level spacing was $\Delta=19 \mu K$. In such systems the linear in time contribution $I_{k i n}^{(2)}(t)$ should be observable for times $t \lesssim 10^{-6} s$. For smaller rings this scale will be even shorter. It is therefore doubtful, that $I_{k i n}^{(2)}$ is experimentally accessible. From this point of view the time independent contribution $I_{t h}^{(2)}$ is more interesting. The presented numerical data indicates, that for frequencies $\varepsilon>\Delta$ there should be a time independent paramagnetic contribution which is comparable in size to the equilibrium persistent current. For small enough samples with discrete spectrum this should in principle be measurable. 
Another important conclusion that can be drawn from the numerical results is the insensitivity of the second order response function to the statistical ensemble. This finding becomes especially interesting in the context of electron-electron interactions. As was pointed out by KY [56], there exists a close connection between this interaction and the non-linear susceptibility. Now the fact that the response function does not depend on whether one works in a canonical or grand canonical ensemble indicates, that effects from electron-electron interactions will not either. This point will be reconsidered and discussed more detailed in the next chapter, where interaction contributions to the persistent current are examined. 


\section{Chapter 5}

\section{Persistent currents and electron-electron interactions}

\subsection{Introduction}

In the previous chapter the dynamic response of a mesoscopic metal ring to an timedependent external flux was studied. The electrons were treated as non-interacting, which is a standard model for disordered electronic systems. One assumes, that Coulomb interaction can be modeled by effective parameters of the system, like e.g. the effective mass or the dephasing time. For example the weak localization correction to the conductivity was calculated for a model of free electrons, and, as mentioned in Chap.2.1, can be fit to experimental results within excellent accuracy. It is therefore natural to first try to explain electronic property of metals in the framework of an independent particle approximation. For mesoscopic metal rings the situation seems to be more complicated. Due to the coherent propagation of electrons around the ring such systems are very sensitive to all effects that influence the particles phase. One consequence of this is the non-linear susceptibility in response to a time-dependent electric field [57,56] that was investigated in Chapter 4 . In Chap. 3.3 electron-electron interactions were investigated by considering the response to a fluctuating field that is caused by the diffusive motion of the background electrons. A strong non-linear susceptibility therefore also indicates the importance of electron-electron interactions in such systems [56]". Indeed, the probably most popular coherence effect in mesoscopic systems, the persistent current, can not be satisfactory explained in a free electron approximation. The seminal experiment by Levy et al. [50] revealed that in the diffusive regime the average current is more than two orders of magnitude lager than the theoretically predicted current from noninteracting electrons [59]. Therefore it seems inevitable to invoke electron-electron

\footnotetext{
${ }^{1} \mathrm{~A}$ relation between external fields and interactions can be formulated generally in a pathintegral description and is in this context known as Hubbard-Stratonovich transformation [36].
} 
interactions to explain the experiment [50]. This was first attempted by Ambegaokar and Eckern (AE) [67], who calculated perturbatively the correction to the average current to first order in the screened Coulomb interaction. They found an average current proportional to $\lambda_{c b} e v_{F} l / L^{2}$, where $\lambda_{c b}$ is a dimensionless measure for some suitably averaged strength of the Coulomb interaction at short wavelengths, $v_{F}$ is the Fermi velocity, $l$ is the elastic mean free path, and $L$ is the circumference of the ring. The precise value of $\lambda_{c b}$ is difficult to estimate, because it is dominated by the non-universal short-wavelength part of the Coulomb interaction. Using [68] $\lambda_{c b} \approx 0.06$, the theoretical current is still a factor of 5 too small to explain the experiment.

The thermodynamic persistent current can be directly calculated in equilibrium from the flux-derivation of the free energy $F(N, \phi)$ or the grand canonical potential $\Omega(\mu, \phi)$, depending on the statistical ensemble. The grand canonical persistent current

$$
I=-c \frac{\partial \Omega(\mu, \phi)}{\partial \phi}
$$

( $c$ is the velocity of light) is usually easier to calculate, since the standard methods of many particle theory presume this ensemble. However, in experiments [50] the rings are isolated and thus have a fixed particle number, which corresponds to a canonical ensemble. In this case the chemical potential $\mu$ becomes a functional of disorder and flux, $\mu \rightarrow \mu(\{U\}, \varphi)$. For non-interacting electrons one can obtain an approximation for the canonical current by expanding $\mu$ to lowest order in the flux [59] (see Eq.(4.67)). The grand canonical current was found to be exponentially small [65]. According to AE [67] this strong ensemble dependence does not apply to interaction effects. AE calculated the first order RPA correction to the current for a grand canonical ensemble and claim that the result is independent of the statistical ensemble. If this is true, one would also expect the susceptibility to be ensemble-independent. And indeed, this is just what was found from the numerical simulations in Chap.4.4. The following calculations will therefore be performed for a grand canonical ensemble. It is also assumed, that the energy spectrum is continuous and the standard diagrammatic disorder averaging can be applied. According to the estimate in Chap.4.5 the results should thus at least be valid at temperatures $T \gtrsim E_{C}$.

The classical (Hartree) contribution to the persistent current has been studied non-perturbatively by Kopietz in Ref. [69]. It was found, that the long range nature of the Coulomb interaction strongly enhances the Hartree contribution. Numerical results presented in Ref. [70] support this finding.

There is still another interaction contribution that has so far been payed no attention to, namely the long wave-length part of the Fock current. To close this gap, the exchange (Fock) contribution to the average current will be re-examined in this chapter. It is well known [20] that for disordered metals singular vertex corrections 
involving so-called Diffusons strongly enhance the effective exchange interaction at small wave-vectors $|\mathbf{q}| \lesssim 2 \pi / l$. This effect has been ignored by AE [67], who focused on the short-wavelength part of the interaction. The possible relevance of these diffusive vertex corrections for persistent currents has been pointed out some time ago by Béal-Monod and Montambaux [72]. However, they also demonstrated that there exists an overall cancellation of the leading infrared singularities. Up until now a quantitative calculation of the persistent current due to exchange interactions with small momentum transfers has not been performed. Here a simple solution of this problem shall be presented.

\subsection{First order RPA corrections to the persistent current}

The considered system is described by the Hamiltonian $\hat{H}=\hat{H}_{0}+\hat{H}_{2}$. (The notation is chosen in analogy to Chap.2.4.) $\hat{H}_{0}$ contains disorder and the static flux $\phi$ through the center of the ring and was already introduced in Eq.(4.3),

$$
\hat{H}_{0}=\sum_{\mathbf{p}} \frac{\mathbf{p}^{2}(\varphi)}{2 m} \hat{\Psi}_{\mathbf{p}}^{\dagger} \hat{\Psi}_{\mathbf{p}}+\int d \mathbf{r} U(\mathbf{r}) \hat{\Psi}^{\dagger}(\mathbf{r}) \hat{\Psi}(\mathbf{r}) .
$$

$\hat{H}_{2}$ describes the electron-electron interaction and is given in Eq.(2.70) as

$$
\hat{H}_{2}=\frac{1}{2} \int d \mathbf{r} d \mathbf{r}^{\prime} f\left(\mathbf{r}-\mathbf{r}^{\prime}\right) \hat{\Psi}^{\dagger}(\mathbf{r}) \hat{\Psi}^{\dagger}\left(\mathbf{r}^{\prime}\right) \hat{\Psi}\left(\mathbf{r}^{\prime}\right) \hat{\Psi}(\mathbf{r})
$$

In Ref. [3] the first order RPA correction to the persistent current was derived by means of a path integral approach. Since so far no path integrals appeared in this work, an alternative derivation using standard diagrammatic expansions is presented here. The grand canonical potential is related to the corresponding statistical operator via

$$
e^{-\beta \Omega}=\operatorname{Sp}\left[e^{-\beta(\hat{H}-\mu \hat{N})}\right]=: Z
$$

where $\hat{N}$ is the particle number operator. For non-interacting electrons the analogous equation reads

$$
e^{-\beta \Omega_{0}}=\operatorname{Sp}\left[e^{-\beta\left(\hat{H}_{0}-\mu \hat{N}\right)}\right]=: Z_{0} .
$$

From $Z / Z_{0}=e^{-\beta\left(\Omega-\Omega_{0}\right)}$ and Eq.(5.1) one thus obtains

$$
I=\frac{c}{\beta} \frac{\partial}{\partial \phi} \ln \left(\frac{Z}{Z_{0}}\right)+I_{0}
$$


$I_{0}=-c\left(\partial \Omega_{0} / \partial \phi\right)$ is the persistent current for non-interacting electrons. $\ln \left(Z / Z_{0}\right)$ can be evaluated exploiting the linked-cluster-theorem which states [21]

$\ln \left(\frac{Z}{Z_{0}}\right)=\ln \left(\langle\hat{U}(\beta, 0)\rangle_{0}\right)=\sum_{n=0}^{\infty} \frac{(-1)^{n}}{n !} \int_{0}^{\beta} d \tau_{1} \cdots d \tau_{n}\left\langle\hat{T}\left[\hat{H}_{2, D}\left(\tau_{1}\right) \cdots \hat{H}_{2, D}\left(\tau_{n}\right)\right]\right\rangle_{0}^{c o n}$

Here the imaginary time Matsubara technique is used and the expectation values are calculated for non-interacting electrons with respect to $H_{0}$. The time-evolution operator is given by

$$
\hat{U}(\beta, 0)=\hat{T} \exp \left[-\int_{0}^{\beta} d \tau \hat{H}_{2, D}(\tau)\right]
$$

where $\hat{T}$ is the time ordering operator and the index $D$ denotes the Dirac picture:

$$
\hat{A}_{D}(\tau)=e^{\hat{H}_{0} \tau} \hat{A} e^{-\hat{H}_{0} \tau}
$$

for an arbitrary operator $\hat{A}$. When expanding the expectation value $\langle\cdots\rangle_{0}^{c o n}$ in Eq.(5.7) by means of the Wick theorem, only connected diagrams are kept, as indicated by con. The Matsubara Green's function $\mathcal{G}_{\mathbf{k}, \mathbf{k}^{\prime}}\left(\tau-\tau^{\prime}\right)$ is defined in Eq.(A.23) (in equilibrium it depends on the time-difference). For non-interacting electrons the function $\mathcal{G}_{\mathbf{k}, \mathbf{k}^{\prime}}^{(0)}\left(\tau-\tau^{\prime}\right)$ is defined analogously, but the expectation value is now calculated with respect to $\hat{H}_{0},\langle\cdots\rangle \rightarrow\langle\cdots\rangle_{0}$. Expanding Eq. (5.7) to first order in the interaction $\hat{H}_{2}$ yields

$$
\begin{aligned}
& \ln \left(\frac{Z}{Z_{0}}\right)^{(1)}=-\frac{1}{\beta} \sum_{\omega_{1}, \omega_{2}} \frac{1}{2 \mathcal{V}} \sum_{\mathbf{q}, \mathbf{k}, \mathbf{k}^{\prime}} f_{\mathbf{q}} {\left[\mathcal{G}_{\mathbf{k}+\mathbf{q}, \mathbf{k}}^{(0)}\left(i \omega_{1}\right) \mathcal{G}_{\mathbf{k}^{\prime}-\mathbf{q}, \mathbf{k}^{\prime}}^{(0)}\left(i \omega_{2}\right)\right.} \\
&\left.-\mathcal{G}_{\mathbf{k}+\mathbf{q}, \mathbf{k}^{\prime}}^{(0)}\left(i \omega_{1}\right) \mathcal{G}_{\mathbf{k}^{\prime}-\mathbf{q}, \mathbf{k}}^{(0)}\left(i \omega_{2}\right)\right] .
\end{aligned}
$$

Diagrammatically this is the contribution from the diagrams (a) and (b) in Fig. 5.1. The first term is the Hartree contribution 5.1(a), and the second one corresponds to the Fock diagram 5.1(b). It will now be shown, that higher order contributions can be resummed, which effectively replaces the bare interaction $f_{q}$ in Fig.5.1(a,b) by the RPA interaction defined in Fig.2.6 (see also Fig.B.1 in App.B). Consider first the Hartree contribution. Higher order terms are produced by simply adding additional 'polarization bubbles' between the two end bubbles. As an example the third order diagram is shown in Fig.5.1(c). The end bubbles can be identified with the Fourier transformed of the non-interacting particle density,

$$
n_{0}(\mathbf{q})=\frac{1}{\beta} \sum_{\omega} \sum_{\mathbf{k}} \mathcal{G}_{\mathbf{k}+\mathbf{q}, \mathbf{k}}^{(0)}(i \omega)
$$


a)

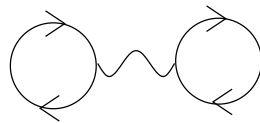<smiles>c1ccc2ccccc2c1</smiles>

b) c)
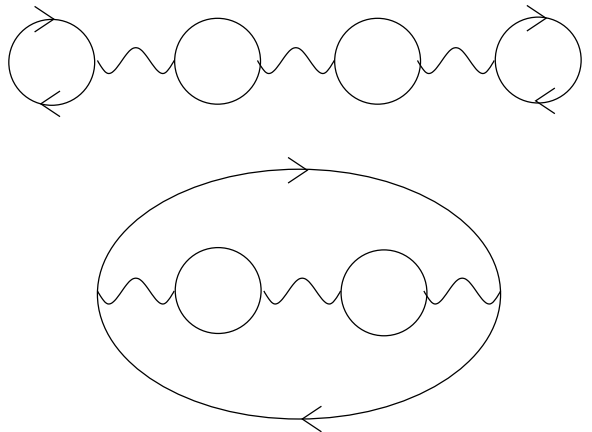

d)

Figure 5.1: Contributions to the expansion of $\ln \left(Z / Z_{0}\right)$ in powers of the interaction. Diagram a): first order Hartree term. Diagram b): first order Fock term. Diagrams c) and $d)$ are the corresponding contributions to third order in $f_{\mathbf{q}}$.

and the 'inner' polarization bubbles have the form $\Pi_{0}\left(\mathbf{q}, \mathbf{q}^{\prime}, i \tilde{\omega}=0\right)$ with

$$
\Pi_{0}\left(\mathbf{q}, \mathbf{q}^{\prime}, i \tilde{\omega}\right)=\frac{1}{\beta} \sum_{\omega} \frac{1}{\mathcal{V}} \sum_{\mathbf{k}, \mathbf{k}^{\prime}} \mathcal{G}_{\mathbf{k}+\mathbf{q}, \mathbf{k}^{\prime}}^{(0)}(i \omega+i \tilde{\omega}) \mathcal{G}_{\mathbf{k}^{\prime}-\mathbf{q}^{\prime}, \mathbf{k}}^{(0)}(i \omega)
$$

where $i \omega$ are Fermionic and $i \tilde{\omega}$ Bosonic Matsubara frequencies. To sum up all RPA-diagrams for the Hartree current, one needs to know the multiplicity of the diagrams. For the $n$-th order there are $\left(\begin{array}{l}n \\ 2\end{array}\right)$ possibilities to choose the vertices for the 'end' bubbles. Since every single interaction vertex consists of two particle creators and two annihilators, there are overall four possible ways to close these bubbles. Now that the two ends are fixed, one can successively attach the remaining vertices to e.g. the left bubble, till the right bubble is reached. The open end of the left vertex can be connected in two different ways to each of the remaining $n-2$ vertices. After the next vertex is linked, the new open end can again be paired with $n-3$ vertices, and so forth. Thus altogether, for $n \geq 3$ there are

$$
4\left(\begin{array}{l}
n \\
2
\end{array}\right) 2(n-2) 2(n-3) \cdots 2(n-(n-1))=2^{n-1} n !
$$

possible pairings. From Eq. (5.7) and the definition of $\hat{H}_{2}$ in Eq.(5.3) one obtains a factor $1 /\left(n ! 2^{n}\right)$ for the $n$-th order contribution to $\ln \left(Z / Z_{0}\right)$. Multiplying this factor with the multiplicity of the corresponding diagram simply yields $1 / 2$ for each order in $f_{\mathbf{q}}$. The result still has to be averaged over disorder. To lowest order in the small parameter $1 /\left(k_{F} l\right)$ (where $k_{F}$ is the Fermi wave vector) disorder correlations between different bubbles are neglected and they are averaged independently. Note that after averaging the polarization is diagonal in wave vector space, $\overline{\Pi_{0}\left(\mathbf{q}, \mathbf{q}^{\prime}, i \tilde{\omega}\right)}=$ $\delta_{\mathbf{q}, \mathbf{q}^{\prime}} \overline{\Pi_{0}}(\mathbf{q}, i \tilde{\omega})$. The over-line denotes disorder averaging. It is then easy to see, that 
the series for the 'inner' bubbles can be summed in exactly the same way as the equation for the effective RPA interaction $f^{\mathrm{RPA}}$ given in Eq.(2.77). Overall one obtains for the Hartree contribution to $\ln \left(Z / Z_{0}\right)$ :

$$
\overline{\ln \left(\frac{Z}{Z_{0}}\right)_{H}}=-\frac{\beta}{2 \mathcal{V}} \sum_{\mathbf{q}} f_{\mathbf{q}}^{\mathrm{RPA}} \overline{n_{0}(\mathbf{q})} \overline{n_{0}(-\mathbf{q})},
$$

with $f_{\mathbf{q}}^{\mathrm{RPA}}:=f_{\mathbf{q}}^{\mathrm{RPA}}(i \tilde{\omega}=0)$. To obtain the multiplicity of the Fock diagrams one can use the same counting scheme as in the Hartree case. However, each vertex is now connected to two other ones and there are no 'end' bubbles. Thus each diagram is counted $n$ times this way, because one can generate the same diagram, independent of which of the $n$ vertices is chosen to be the 'left end'. The multiplicity of the Fock diagrams is therefore just the Hartree multiplicity divided by $n$. Each Fock term in the expansion of $\ln \left(Z / Z_{0}\right)$ thus obtains a factor $1 /(2 n)$ instead of the $1 / 2$ for the Hartree terms. Averaging over disorder hence yields

$$
\overline{\ln \left(\frac{Z}{Z_{0}}\right)_{F}^{(n)}}=\frac{1}{2 n} \sum_{i \tilde{\omega}} \sum_{\mathbf{q}}\left[f_{\mathbf{q}} \overline{\Pi_{0}}(\mathbf{q}, i \tilde{\omega})\right]^{n} .
$$

This structure is familiar form the power series of $-\ln (1-x)$ and the terms can be summed to

$$
\overline{\ln \left(\frac{Z}{Z_{0}}\right)_{F}}=-\frac{1}{2} \sum_{i \tilde{\omega}} \sum_{\mathbf{q}} \ln \left[1-f_{\mathbf{q}} \overline{\Pi_{0}}(\mathbf{q}, i \tilde{\omega})\right] .
$$

Inserting the above results into Eq.(5.6) one obtains $I=I_{0}+I_{H}+I_{F}$, where $I_{0}$ is the non-interacting persistent current, and

$$
\begin{aligned}
\bar{I}_{H} & =-\frac{c}{2} \frac{\partial}{\partial \phi} \frac{1}{\mathcal{V}} \sum_{\mathbf{q}} f_{\mathbf{q}}^{\mathrm{RPA}} \overline{n_{0}(\mathbf{q})} \overline{n_{0}(-\mathbf{q})} \\
\bar{I}_{F} & =-\frac{c}{2} \sum_{\mathbf{q}} \frac{1}{\beta} \sum_{\tilde{\omega}} f_{\mathbf{q}}^{\mathrm{RPA}}(i \tilde{\omega}) \frac{\partial}{\partial \phi} \overline{\Pi_{0}}(\mathbf{q}, i \tilde{\omega}) .
\end{aligned}
$$

The effective interaction is connected to the bare one by $f^{\mathrm{RPA}}=\left(1-f^{-1} \Pi_{0}\right)^{-1}$ (see Eq.(2.77)), which is just the factor obtained by differentiating the logarithm in Eq.(5.16). Note that the Hartree contribution $I_{H}$ involves only the static part $f_{\mathbf{q}}^{\mathrm{RPA}} \equiv f_{\mathbf{q}}^{\mathrm{RPA}}(0)$ of the screened interaction.

\subsection{The long wave-length contribution to the Fock current}

Now the average Fock current $\bar{I}_{F}$ in the diffusive regime will be more closely examined. To evaluate $\bar{I}_{F}$ one needs to know the flux-derivative of the average polariza- 

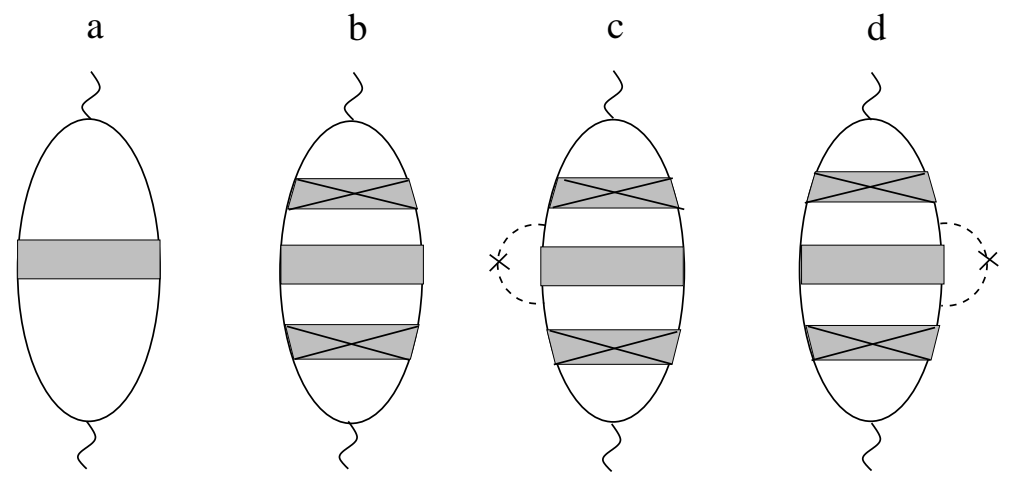

Figure 5.2: Diagrams contributing to $\partial \bar{\Pi}_{0}(\mathbf{q}, i \tilde{\omega}) / \partial \phi$. Retaining only (a) reproduces the result of $A E$. The plain shaded box represent the Cooperon, the solid lines are average non-interacting Green's functions, and the small wavy lines denote density vertices. (b) Vertex correction with two additional Diffusons (represented by shaded stripes with a cross). For small $\mathbf{q}$ and $\tilde{\omega}$ this diagram is much larger than (a). However, to leading order in $\left(k_{F} l\right)^{-1}(b)$ is canceled by (c) and (d). Here the dashed lines denote the impurity average $\overline{U_{\mathbf{q}} U_{-\mathbf{q}}}$.

tion $\partial \bar{\Pi}_{0}(\mathbf{q}, i \omega) / \partial \phi$. AE [67] have approximated the leading flux dependence of the polarization by retaining only diagram (a) in Fig.5.2. This yields

$$
\begin{array}{r}
\overline{\Pi_{0, A E}}(\mathbf{q}, i \tilde{\omega})=\frac{1}{\beta} \sum_{\omega} \frac{1}{\mathcal{V}} \sum_{\mathbf{k}, \mathbf{p}} \overline{\mathcal{G}}^{0}{ }_{\mathbf{p}}(i \omega+i \tilde{\omega}) \overline{\mathcal{G}}_{\mathbf{p}-\mathbf{q}}(i \omega) \mathrm{C}_{0}(\mathbf{k}, i \tilde{\omega}) \\
\times \overline{\mathcal{G}}_{\mathbf{k}-\mathbf{p}+\mathbf{q}}(i \omega+i \tilde{\omega}) \overline{\mathcal{G}}_{\mathbf{k}-\mathbf{p}}(i \omega) .
\end{array}
$$

The Cooperon is dominated by small momenta $|\mathbf{k}| \ll 1 / l$, so that the $\mathbf{k}$ dependence of the Green's functions can be neglected:

$$
\begin{gathered}
\overline{\Pi_{0, A E}}(\mathbf{q}, i \tilde{\omega}) \approx \frac{1}{\beta} \sum_{\omega} \frac{1}{\mathcal{V}} \sum_{\mathbf{p}}{\overline{\mathcal{G}^{0}}}_{\mathbf{p}}(i \omega+i \tilde{\omega}) \overline{\mathcal{G}}^{0}{ }_{\mathbf{p}-\mathbf{q}}(i \omega) \overline{\mathcal{G}}^{0}{ }_{\mathbf{p}-\mathbf{q}}(i \omega+i \tilde{\omega}) \overline{\mathcal{G}}_{\mathbf{p}}{ }_{\mathbf{p}}(i \omega) \\
\times \sum_{\mathbf{k}}^{\prime} \mathrm{C}_{0}(\mathbf{k}, i \tilde{\omega})
\end{gathered}
$$

The prime indicates, that the $\mathbf{k}$-sum is restricted to $|\mathbf{k}|<1 / l$. Inserting Eq.(5.20) into Eq.(5.18) leads to

$$
\begin{aligned}
I_{F, A E}=\frac{-c}{2} \frac{\partial}{\partial \phi} & \frac{1}{\mathcal{V}} \sum_{\mathbf{q}, \mathbf{p}} \frac{1}{\beta^{2}} \sum_{\tilde{\omega}, \omega} f_{\mathbf{q}}^{\mathrm{RPA}}(i \tilde{\omega}) \overline{\mathcal{G}^{0}}{ }_{\mathbf{p}}(i \omega+i \tilde{\omega}) \overline{\mathcal{G}^{0}}{ }_{\mathbf{p}-\mathbf{q}}(i \omega) \overline{\mathcal{G}^{0}}{ }_{\mathbf{p}-\mathbf{q}}(i \omega+i \tilde{\omega}) \overline{\mathcal{G}^{0}}{ }_{\mathbf{p}}(i \omega) \\
& \times \sum_{\mathbf{k}}^{\prime} \mathrm{C}_{0}(\mathbf{k}, i \tilde{\omega}) .
\end{aligned}
$$


This is the Fock contribution evaluated by $\mathrm{AE}^{2}$

For $|\mathbf{q}| \ll k_{F}$ and $|\tilde{\omega}| \tau_{e l} \ll 1$ the polarization given in Eq.(5.20) can be evaluated, yielding

$$
\frac{\partial}{\partial \phi} \bar{\Pi}_{0}(\mathbf{q}, i \tilde{\omega})_{A E}=\nu|\tilde{\omega}| \tau_{e l} \frac{\partial}{\partial \phi} g_{W L}(i \tilde{\omega}, \phi)
$$

$g_{W L}(i \tilde{\omega}, \phi)$ is the well known weak-localization correction to the dimensionless average conductivity $\sigma(i \tilde{\omega})$ in units of the corresponding Drude conductivity $\sigma_{0}$,

$$
\begin{gathered}
\sigma(i \tilde{\omega})=\sigma_{0}\left[1+g_{W L}(i \tilde{\omega}, \phi)\right], \\
g_{W L}(i \tilde{\omega}, \phi)=-\frac{2 \Delta}{\pi} \sum_{\mathbf{Q}}^{\prime} \frac{1}{D \mathbf{Q}^{2}+|\tilde{\omega}|+1 / \tau_{\varphi}} .
\end{gathered}
$$

Here the $x$-component of $\mathbf{Q}$ is quantized according to $Q_{x}=\frac{2 \pi}{L}\left(n+2 \phi / \phi_{0}\right)$.

Clearly, the approximation (5.22) cannot be correct for $|\mathbf{q}| l \ll 1$, because then the density vertices in Fig.5.2(a) can be dressed by singular Diffuson corrections, as shown in Figs.5.2(b)-(d). As a consequence, this regime requires a special treatment. This has already been noticed by Béal-Monod and Montambaux [72]. Unfortunately, the sum of diagrams (b)-(d) in Fig. 5.2 cancels to leading order in $\left(k_{F} l\right)^{-1}$ [72], so that at the first sight it seems that the calculation of the leading non-vanishing behavior of $\partial \bar{\Pi}_{0}(\mathbf{q}, i \tilde{\omega}) / \partial \phi$ requires rather complicated mathematical manipulations. However, there is a simple way to avoid this technical complication: As shown in Ref. [71], current conservation implies that for small wave-vectors and frequencies the polarization is of the form

$$
\bar{\Pi}_{0}(\mathbf{q}, i \tilde{\omega})=\nu \frac{\tilde{D}(i \tilde{\omega}) \mathbf{q}^{2}}{\tilde{D}(i \tilde{\omega}) \mathbf{q}^{2}+|\tilde{\omega}|}
$$

where $\tilde{D}(i \tilde{\omega})$ is the generalized frequency-dependent diffusion coefficient, which is related to the frequency-dependent average conductivity $\sigma(i \tilde{\omega})$ via [71]

$$
\frac{\tilde{D}(i \tilde{\omega})}{D}=\frac{\sigma(i \tilde{\omega})}{\sigma_{0}}
$$

Combining Eq.(5.26) with Eqs.(5.23) and (5.24), it is concluded that

$$
\frac{\partial}{\partial \phi} \tilde{D}(i \tilde{\omega})=\frac{\partial}{\partial \phi} D\left[1+g_{W L}(i \tilde{\omega}, \phi)\right]=D \frac{\partial}{\partial \phi} g_{W L}(i \tilde{\omega}, \phi) .
$$

\footnotetext{
${ }^{2}$ see Eq.6 in Ref. [67]. AE have normalized the Cooperon using the three dimensional density of states $\nu_{3}=1 /(\Delta \mathcal{V})$ instead of $\Delta$, which gives rise to an extra factor $1 / \mathcal{V}$ in their notation.
} 
From Eqs.(5.25) and (5.27) it is now obvious that the flux-dependence of the average polarization can be expressed in terms of the weak localization correction (5.24) to the dynamic conductivity. A straightforward calculation yields $\left(\nu=\nu_{3}\right)$

$$
\begin{aligned}
\frac{\partial}{\partial \phi} \bar{\Pi}_{0}(\mathbf{q}, i \tilde{\omega}) & =\nu\left[\frac{\frac{\partial}{\partial \phi} \tilde{D}(i \tilde{\omega}) \mathbf{q}^{2}}{\tilde{D}(i \tilde{\omega}) \mathbf{q}^{2}+|\tilde{\omega}|}-\frac{\tilde{D}(i \tilde{\omega}) \mathbf{q}^{2} \frac{\partial}{\partial \phi} \tilde{D}(i \tilde{\omega}) \mathbf{q}^{2}}{\left(\tilde{D}(i \tilde{\omega}) \mathbf{q}^{2}+|\tilde{\omega}|\right)^{2}}\right] \\
& =\nu \frac{|\tilde{\omega}| D \mathbf{q}^{2}}{\left(D \mathbf{q}^{2}\left[1+g_{W L}(i \tilde{\omega}, \phi)\right]+|\tilde{\omega}|\right)^{2}} \frac{\partial}{\partial \phi} g_{W L}(i \tilde{\omega}, \phi) .
\end{aligned}
$$

This expression, which is one of the main results of this chapter, is valid for systems with continuous spectrum $\left(1 / \tau_{\varphi}>\Delta\right)$ in the semiclassical regime $|\mathbf{q}| \lesssim 2 \pi / l$ and $|\tilde{\omega}| \lesssim \tau_{e l}^{-1}$. Note that for $|\mathbf{q}| l=O(1)$ Eq. $(5.28)$ smoothly matches the short wavelength result (5.22). In fact, using $D=v_{F} l / 3$, it is seen that for $\left|g_{W L}\right| \ll 1$ both expressions are identical at $|\mathbf{q}| l=\sqrt{3}$. On the other hand, in the regime $|\mathbf{q}| l \ll 1$ the long-wavelength result Eq.(5.28) is a factor of $(\mathbf{q} l)^{-2}$ larger than Eq.(5.22).

To see whether this infrared enhancement is sufficient to lead to a significant exchange contribution to the persistent current, Eq.(5.28) is substituted into Eq.(5.18). This way one obtains

$$
\begin{aligned}
\bar{I}_{F}^{l o n g} & =-\frac{c}{2} T \sum_{\tilde{\omega}} \sum_{\mathbf{q}}^{\prime} \frac{f_{\mathbf{q}} \frac{\partial}{\partial \phi} \bar{\Pi}_{0}(\mathbf{q}, i \tilde{\omega})}{1+f_{\mathbf{q}} \bar{\Pi}_{0}(\mathbf{q}, i \tilde{\omega})} \\
& \approx-\frac{c}{2} T \sum_{n} \sum_{\mathbf{q}}^{\prime} \frac{|\tilde{\omega}|}{\left(D \mathbf{q}^{2}\left[1+g_{W L}(i \tilde{\omega}, \phi)\right]+|\tilde{\omega}|\right)} \frac{\frac{\partial}{\partial \phi} g_{W L}(i \tilde{\omega}, \phi)}{\left(1+g_{W L}(i \tilde{\omega}, \phi)\right)}
\end{aligned}
$$

for the long-wavelength Fock contribution to the average current. The second line in Eq. (5.29) is valid in the experimentally relevant limit $\left|f_{\mathbf{q}} \bar{\Pi}_{0}(\mathbf{q}, i \tilde{\omega})\right| \gg 1$. Note that the current (5.29) increases with the strength of the disorder, because the (negative) weak-localization correction $g_{W L}$ in the denominator of Eq. (5.29) becomes more and more important with increasing disorder, thus reducing the screening. Of course, the above calculation is only controlled for $\left|g_{W L}\right| \ll 1$, so that from now on this correction will be ignored. For simplicity it is assumed that the ring is sufficiently thin, such that only the motion along the circumference is diffusive. In this case the q-sum in the square brace of Eq.(5.29) can be replaced by a one-dimensional integral, which for $L \gg l$ is independent of the ultraviolet cutoff.

$$
\begin{aligned}
\bar{I}_{F}^{l o n g} & =-\frac{c}{2} T \sum_{\tilde{\omega}} \sum_{\mathbf{q}}^{\prime} \frac{|\tilde{\omega}|}{D \mathbf{q}^{2}+|\tilde{\omega}|} \frac{\partial}{\partial \phi} g_{W L}(i \tilde{\omega}, \phi) \\
& \approx-\frac{c}{2} T \sum_{\tilde{\omega}} \frac{L}{2 \pi} \int_{-\infty}^{\infty} d q \frac{|\tilde{\omega}|}{D q^{2}+|\tilde{\omega}|} \frac{\partial}{\partial \phi} g_{W L}(i \tilde{\omega}, \phi) .
\end{aligned}
$$


One obtains

$$
\bar{I}_{F}^{l o n g}=-\frac{c}{4} T \sum_{\tilde{\omega}}\left[\frac{|\tilde{\omega}| L^{2}}{D}\right]^{1 / 2} \frac{\partial}{\partial \phi} g_{W L}(i \tilde{\omega}, \phi) .
$$

The dimensionless weak-localization correction is evaluated for a thin ring by decomposing it into a Fourier series.

$$
\begin{aligned}
g_{W L}(i \tilde{\omega}, \phi) & \approx-\frac{2 \Delta}{\pi} \sum_{Q_{x}}^{\prime} \frac{1}{D Q_{x}^{2}+|\tilde{\omega}|+1 / \tau_{\varphi}} \\
& \approx-\frac{2 \Delta}{\pi} \sum_{n=-\infty}^{\infty} \frac{1}{4 \pi^{2} E_{c}\left(n+2 \phi / \phi_{0}\right)^{2}+|\tilde{\omega}|+1 / \tau_{\varphi}} \\
& =:-\frac{2 \Delta}{\pi}\left[\frac{P_{0}}{2}+\sum_{j=1}^{\infty} P_{j} \cos \left(4 \pi j \phi / \phi_{0}\right)\right] .
\end{aligned}
$$

Here the restriction $|\mathbf{Q}|<1 / l$ was dropped, since the sum falls of fast enough. The Fourier components $P_{j}$ are given by

$$
\begin{aligned}
P_{j} & =\frac{4}{\phi_{0}} \int_{0}^{\phi_{0} / 2} d \phi \sum_{n=-\infty}^{\infty} \frac{\cos \left(4 \pi j \phi / \phi_{0}\right)}{4 \pi^{2} E_{c}\left(n+2 \phi / \phi_{0}\right)^{2}+|\tilde{\omega}|+1 / \tau_{\varphi}} \\
& =2 \int_{-\infty}^{\infty} d \xi \frac{\cos (2 \pi j \xi)}{4 \pi^{2} E_{c} \xi^{2}+|\tilde{\omega}|+1 / \tau_{\varphi}}=\frac{e^{-j \sqrt{\frac{|\tilde{\omega}|+1 / \tau_{\varphi}}{E_{c}}}}}{\sqrt{E_{c}} \sqrt{|\tilde{\omega}|+1 / \tau_{\varphi}}} .
\end{aligned}
$$

Since $E_{c}=D / L^{2} \gg \Delta$ the current $\bar{I}_{F}^{l o n g}$ in Eq.(5.31) is dominated by frequencies $|\tilde{\omega}| \gg \Delta$ and one can thus neglect $1 / \tau_{\varphi}$ in Eq.(5.33) without violating the condition $1 / \tau_{\varphi}>\Delta$. The current is thus given by

$$
\bar{I}_{F}^{l o n g}=-\frac{c}{4} \frac{2 \Delta}{\pi} T \sum_{\tilde{\omega}}\left[\frac{|\tilde{\omega}|}{E_{c}}\right]^{1 / 2} \sum_{j=1}^{\infty} \frac{4 \pi j}{\phi_{0}} \frac{e^{-j \sqrt{|\tilde{\omega}| / E_{c}}}}{\sqrt{|\tilde{\omega}| E_{c}}} \sin \left(4 \pi j \phi / \phi_{0}\right) .
$$

For $T \rightarrow 0$ the summation over the Matsubara frequencies can be performed exactly by replacing $T \sum_{\tilde{\omega}} \rightarrow 1 /(2 \pi) \int d \tilde{\omega}$ :

$$
\bar{I}_{F}^{\text {long }}=-\frac{c \Delta}{\pi \phi_{0} E_{c}} \int_{-\infty}^{\infty} d \tilde{\omega} \sum_{j=1}^{\infty} j e^{-j \sqrt{|\tilde{\omega}| / E_{c}}} \sin \left(4 \pi j \phi / \phi_{0}\right) .
$$

Evaluating the $\tilde{\omega}$-integral results in

$$
\int_{-\infty}^{\infty} d \tilde{\omega} e^{-j \sqrt{|\tilde{\omega}| / E_{c}}}=\frac{4 E_{c}}{j^{2}},
$$


and one arrives at

$$
\bar{I}_{F}^{\text {long }}=-\frac{4 c \Delta}{\pi \phi_{0}} \sum_{j=1}^{\infty} \frac{1}{j} \sin \left(4 \pi j \phi / \phi_{0}\right) .
$$

Inserting the explicit form of the three dimensional level spacing $\Delta=\pi^{2} /\left(\mathcal{V} m k_{F}\right)$ and the flux quantum $\phi_{0}=2 \pi c / e$, the long-wavelength Fock contribution to the current can finally be expressed as

$$
\bar{I}_{F}^{l o n g}=-\frac{e v_{F}}{L} \frac{\pi}{\left(k_{F} a\right)^{2}} f(\phi),
$$

where $a$ is the transverse thickness of the ring, and

$$
\begin{aligned}
f(\phi) & =\frac{2}{\pi} \sum_{j=1}^{\infty} \frac{\sin \left(4 \pi j \phi / \phi_{0}\right)}{j} \\
& =1-4 \phi / \phi_{0} \quad, \quad \text { for } 0<\phi / \phi_{0}<1 / 2 .
\end{aligned}
$$

Comparing $\bar{I}_{F}^{\text {long }}$ with the the non-interacting current at constant particle number [58, 59] given in Eqs.(4.67),(4.68) one surprisingly finds, that both have the same order of magnitude. In the experimentally relevant parameter regime [50], this current is smaller than the current due to the short wavelength part of the Coulomb interaction calculated by $\mathrm{AE}$ 67].

In summary, a quantitative calculation of the long-wavelength exchange contribution to the average persistent current in mesoscopic metal rings was presented. The current has the same order of magnitude as the non-interacting persistent current $I(\varphi)$ at constant particle number. Also the leading weak localization correction to the average polarization $\bar{\Pi}_{0}(\mathbf{q}, \tilde{\omega})$ was calculated in the regime $|\mathbf{q}| \lesssim 2 \pi / l$ and $\Delta \lesssim|\tilde{\omega}| \ll \tau_{e l}^{-1}$, effectively taking singular Diffuson corrections to the density vertices into account. 


\section{Chapter 6}

\section{Summary}

The first part of this work (Chapter 2 and 3) was devoted to dephasing effects in disordered metallic conductors. In accordance with experiments, the dephasing rate $1 / \tau_{\varphi}$ was extracted from the weak localization correction to the conductivity and therewith from the cutoff in the Cooperon propagator. A novel Eikonal approach was introduced. This method provides a general formalism to calculate the Cooperon and the Diffuson in the presence of dephasing fields. It is non-perturbative since infinite orders in the field are included. An explicit formula describing both propagators for arbitrary external fields was derived. This result was then applied to investigate two important sources of dephasing: Nyquist noise $\left(1 / \tau_{n n}\right)$ and external microwave radiation $\left(1 / \tau_{A C}\right)$. In the first case, dephasing is caused by the diffusive motion of the background electrons in the sample and the fluctuation-dissipation theorem was used to obtain the correlator of the corresponding random electric field. This way the frequency- and temperature-dependence of $1 / \tau_{n n}(T, \varepsilon)$ is determined unambiguously without the need of any further cutoffs. By rederiving the conventional results for the temperature dependence [22,8,32], the physically motivated cutoff procedure used in earlier works could thus be justified a posteriori. The frequency dependence of $1 / \tau_{n n}$ was calculated for macroscopic and mesoscopic systems in dimensions $d \leq 2$. These results have apparently not been derived previously. Dephasing due to external microwave radiation has previously only been studied for spatially constant fields [22]. The Eikonal approximation allows also to investigate dephasing-fields with finite wave-length $\mathbf{q}_{0}$ that induce density fluctuations in the metallic sample. It was shown, that there exists a parameter regime, where $1 / \tau_{A C}$ is indeed dominated by the spatial variation of the external radiation and the result is qualitatively different from the case $\mathbf{q}_{0}=0$. The theoretical predictions are in agreement with experimental data from Refs. [42, 46]. Without further effort the Eikonal method can also be applied to calculate the cutoff $1 / \tau_{D}$ in the Diffuson. This was outlined in the last section of Chapter 3. Known results for $1 / \tau_{D}$ in infinite systems are conveniently reproduced and new results for mesoscopic samples are obtained. The Eikonal formalism can in principle be applied to all kinds of fluctuating longitudinal fields. Recently dephasing 
due to $1 / f$-noise has been studied within the semiclassical path-integral approach [37. Here again a phenomenological cutoff has to be introduced and it should be possible to circumvent this ambiguities within the Eikonal approach.

In the remaining chapters (Chapter 1 and 5) the non-linear response of mesoscopic metal rings and the closely related problem of electron-electron interactions was examined. Motivated by the experimental progress to decrease the size of probes, the response of systems with discrete energy spectrum to a time dependent field is analyzed. The usual Green's function approach yields a time independent current that is induced by the oscillating field in second order. It has recently been argued, that it is such a current, generated by external noise, rather than a true equilibrium current that is measured in the persistent current experiments [66]. By exact manipulations the corresponding second order response kernel was shown to contain an infinite term. This divergence is caused by an incorrect treatment of the switching on procedure. Since diagrammatic averaging still produces a finite value, it can not be applied in the discrete spectrum limit. By simply applying Fermi's golden rule it is outlined, that after a correct treatment the seemingly divergent term gives rise to an induced current that grows linearly in time, even though the external driving field oscillates with frequency $\varepsilon$. This result is however only valid on a short times scale $t<\Delta^{-1}$. Beside this, there still is a time-independent contribution $I_{t h}$ to the current. Careful numerical simulations revealed that this current has the same sign as the canonical equilibrium current for non-interacting electrons but, depending on the oscillating external flux, may be lager by one order of magnitude. Using a simple estimate, the spectrum is predicted to become continuous for temperatures below the Thouless energy $E_{c}$. In the persistent current experiment by Mohanty et al. [55] the temperature was still of order $E_{c}$ and it is not clear whether the spectrum has a discrete part or not. For smaller rings of nano-meter size [48] $I_{t h}$ should in principle be measurable. As expected it turned out, that the second order response function is independent of the underlying statistical ensemble. Due to the close relation between non-linear response and electron-electron interactions [36, 56] this is in agreement with the assumption that interaction corrections to the canonical persistent current $I(\varphi)$ can be calculated for a fixed chemical potential [67]. This idea was revisited in the last chapter, where a simple way to calculate the long wave-length Fock contribution to the persistent current was presented. Quantitative results for this regime have not been derived previously. It turned out, that exchange interactions can not explain the experimentally measured current. This justifies the assumption made in Refs. [69, 75] where it was argued, that the Fock current can be neglected while the classical (Hartree) contribution to the persistent current is strongly enhanced due to the long-range nature of the bare Coulomb interaction. 


\section{Appendix A}

\section{How higher order response functions can be calculated within the Matsubara technique}

The system considered here consists of non-interacting electrons in an external, time dependent potential $V(\mathbf{r}, t)$. It is described by the Hamiltonian

$$
\hat{H}=\hat{H}_{0}+\hat{H}_{1}(t)
$$

The time independent one-particle Hamiltonian $\hat{H}_{0}$ contains all static potentials, e.g. disorder. In the following, an index ' 0 ' will denote equilibrium-quantities determined by $\hat{H}_{0}$. The time-dependent potential is given by

$$
\hat{H}_{1}(t):=\sum_{\mathbf{q}, \mathbf{q}^{\prime}} V_{\mathbf{q}, \mathbf{q}^{\prime}}(t) \hat{\Psi}_{\mathbf{q}}^{\dagger} \hat{\Psi}_{\mathbf{q}^{\prime}}
$$

and $V_{\mathbf{q}, \mathbf{q}^{\prime}}(t)=\left\langle\mathbf{q}\left|\hat{H}_{1}(t)\right| \mathbf{q}^{\prime}\right\rangle=\frac{1}{\mathcal{V}} V_{\mathbf{q}-\mathbf{q}^{\prime}}(t)$ is the Fourier-transform of the potential $V(\mathbf{r}, t)$. It is assumed, that the external field has been switched on adiabatically. $\hat{\Psi}_{\mathbf{q}}^{\dagger}$ and $\hat{\Psi}_{\mathbf{q}}$ are the creation- and annihilation operators for a particle in momentum-state q.

\section{A.1 The real time response function}

To calculate real-time response functions to arbitrary order in $V$ it is convenient to use the Keldysh-technique. To be more concrete, consider these two examples: The paramagnetic contribution to the current and the particle-density. In momentum- 
time representation they can be expressed as

$$
\begin{gathered}
\mathbf{j}_{\text {para }}(\mathbf{k}, t)=-\frac{e}{2 m} \sum_{\mathbf{q}}(\mathbf{k}+2 \mathbf{q})\left\langle\hat{\Psi}_{H, \mathbf{k}+\mathbf{q}}^{\dagger}(t) \hat{\Psi}_{H, \mathbf{q}}(t)\right\rangle \\
=-\frac{e}{2 m} \sum_{\mathbf{q}}(\mathbf{k}+2 \mathbf{q}) \frac{1}{i} \mathcal{G}_{\mathbf{q}, \mathbf{k}+\mathbf{q}}^{<}(t, t), \\
n(\mathbf{k}, t)=\sum_{\mathbf{q}}\left\langle\hat{\Psi}_{H, \mathbf{k}+\mathbf{q}}^{\dagger}(t) \hat{\Psi}_{H, \mathbf{q}}(t)\right\rangle=\sum_{\mathbf{q}} \frac{1}{i} \mathcal{G}_{\mathbf{q}, \mathbf{k}+\mathbf{q}}^{<}(t, t) .
\end{gathered}
$$

The index $H$ denotes time-evolution in the Heisenberg picture. The Green's functions are defined by

$$
\mathcal{G}_{\mathbf{k}, \mathbf{k}^{\prime}}^{<}\left(t, t^{\prime}\right)=i\left\langle\hat{\Psi}_{H, \mathbf{k}^{\prime}}^{\dagger}\left(t^{\prime}\right) \hat{\Psi}_{H, \mathbf{k}}(t)\right\rangle ; \mathcal{G}_{\mathbf{k}, \mathbf{k}^{\prime}}^{>}\left(t, t^{\prime}\right)=-i\left\langle\hat{\Psi}_{H, \mathbf{k}}(t) \hat{\Psi}_{H, \mathbf{k}^{\prime}}^{\dagger}\left(t^{\prime}\right)\right\rangle
$$

and

$$
\mathcal{G}_{\mathbf{k}, \mathbf{k}^{\prime}}^{K}\left(t, t^{\prime}\right)=\mathcal{G}_{\mathbf{k}, \mathbf{k}^{\prime}}^{<}\left(t, t^{\prime}\right)+\mathcal{G}_{\mathbf{k}, \mathbf{k}^{\prime}}^{>}\left(t, t^{\prime}\right) .
$$

For equal times $t=t^{\prime}$ one can rewrite $\mathcal{G}^{K}$ as

$$
\begin{aligned}
\mathcal{G}_{\mathbf{k}, \mathbf{k}^{\prime}}^{K}(t, t) & =2 \mathcal{G}_{\mathbf{k}, \mathbf{k}^{\prime}}^{<}(t, t)+\left(\mathcal{G}_{\mathbf{k}, \mathbf{k}^{\prime}}^{>}(t, t)-\mathcal{G}_{\mathbf{k}, \mathbf{k}^{\prime}}^{<}(t, t)\right) \\
& =2 \mathcal{G}_{\mathbf{k}, \mathbf{k}^{\prime}}^{<}(t, t)-i\left(\left\langle\hat{\Psi}_{H, \mathbf{k}}(t) \hat{\Psi}_{H, \mathbf{k}^{\prime}}^{\dagger}(t)\right\rangle+\left\langle\hat{\Psi}_{H, \mathbf{k}^{\prime}}^{\dagger}(t) \hat{\Psi}_{H, \mathbf{k}}(t)\right\rangle\right) \\
& =2 \mathcal{G}_{\mathbf{k}, \mathbf{k}^{\prime}}^{<}(t, t)-i\left\langle U_{H}^{\dagger}(t)\left[\hat{\Psi}_{\mathbf{k}} \hat{\Psi}_{\mathbf{k}^{\prime}}^{\dagger}+\hat{\Psi}_{\mathbf{k}^{\prime}}^{\dagger} \hat{\Psi}_{\mathbf{k}}\right] U_{H}(t)\right\rangle \\
& =2 \mathcal{G}_{\mathbf{k}, \mathbf{k}^{\prime}}^{<}(t, t)-i \delta_{\mathbf{k}, \mathbf{k}^{\prime}} .
\end{aligned}
$$

Thus all time-dependent non-equilibrium properties of the quantities in Eqs.(A.3), (A.4) are entirely determined by the Keldysh component $\mathcal{G}^{K}$ of the Green's function. Calculating e.g. the current or density response functions is therefore equivalent to calculating the response function for $\mathcal{G}^{K}(t, t)$.

In the Keldysh formulation the Green's function is represented as a matrix

$$
\underline{G}=\left(\begin{array}{cc}
\mathcal{G}^{R} & \mathcal{G}^{K} \\
0 & \mathcal{G}^{A}
\end{array}\right)
$$

$G^{R}$ and $G^{A}$ are the retarded and advanced Green's function. For non-interacting particles in an external field $V(\mathbf{r}, t)$ the Green's function can be expressed through the Dyson equation

$$
\underline{G}_{\mathbf{k}, \mathbf{k}^{\prime}}\left(t, t^{\prime}\right)=\underline{G}_{\mathbf{k}, \mathbf{k}^{\prime}}^{0}+\sum_{\mathbf{q}, \mathbf{q}^{\prime}} \int_{-\infty}^{\infty} d t_{1} \underline{G}_{\mathbf{k}, \mathbf{q}}^{0}\left(t, t_{1}\right) V_{\mathbf{q}, \mathbf{q}^{\prime}}\left(t_{1}\right) \underline{G}_{\mathbf{q}^{\prime}, \mathbf{k}^{\prime}}\left(t_{1}, t^{\prime}\right) .
$$


Here $\underline{G}^{0}$ is the Green's function without external field. In equilibrium the Green's function depends only on the time-difference $\underline{G}^{0}\left(t, t^{\prime}\right)=\underline{G}^{0}\left(t-t^{\prime}\right)$ and the Keldysh component is related to the retarded and advanced functions via

$$
\mathcal{G}^{0, K}(\omega)=\int_{-\infty}^{\infty} d t e^{i \omega t} \mathcal{G}^{0, K}(t)=\tanh \left(\frac{\omega \beta}{2}\right)\left[\mathcal{G}^{0, R}(\omega)-\mathcal{G}^{0, A}(\omega)\right]
$$

with $\beta=1 / T$. Since the response to the perturbation $\hat{H}_{1}(t)$ is determined by the Keldysh component at equal times, the function (note the factor 2 in Eq.(A.7))

$$
g_{\mathbf{k}, \mathbf{k}^{\prime}}(t):=\frac{1}{2 i} \mathcal{G}_{\mathbf{k}, \mathbf{k}^{\prime}}^{K}(t, t)
$$

is defined. Its Fourier transform is given by (momentum-variables are suppressed)

$$
\begin{aligned}
g(\omega) & =\int_{-\infty}^{\infty} d t e^{i \omega t} g(t)=\frac{1}{2 i} \int_{-\infty}^{\infty} \frac{d \varepsilon}{2 \pi} \mathcal{G}^{K}(\varepsilon, \varepsilon-\omega) \\
& =\frac{1}{2 i} \int_{-\infty}^{\infty} \frac{d \varepsilon}{2 \pi} \operatorname{Sp}\left[\underline{\sigma}_{1} \underline{G}(\varepsilon, \varepsilon-\omega)\right]
\end{aligned}
$$

where

$$
\underline{\sigma}_{1}=\left(\begin{array}{ll}
0 & 1 \\
1 & 0
\end{array}\right) .
$$

The Fourier transformation for two time-variables is defined as

$$
\mathcal{G}^{K}\left(\omega, \omega^{\prime}\right)=\int_{-\infty}^{\infty} d t d t^{\prime} e^{i\left(\omega t-\omega^{\prime} t^{\prime}\right)} \mathcal{G}^{K}\left(t, t^{\prime}\right)
$$

Diagrammatic representations for the first and n-th order contribution to $g(\omega)$ are

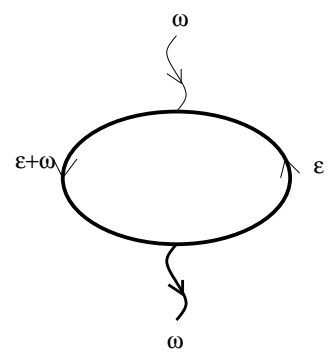

first Order

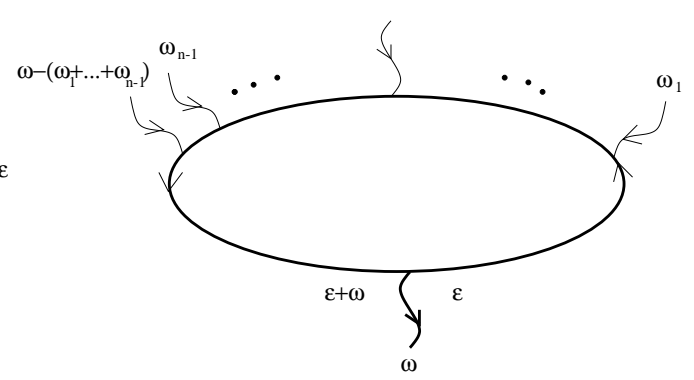

n-th Order

Figure A.1: Diagrams for the first and $n$-th Order contribution to $g(\omega)$. Thin wiggled lines symbolize the external field. The thick wiggled line is the w-vertex. Solid lines represent $\underline{G}^{0}$.

shown in Fig.A.1. In the following momentum-variables are suppressed and it has 
to be kept in mind, that it is summed over all internal momenta. The first order contribution is then given by

$$
g^{(1)}(\omega)=\frac{1}{2 i} V(\omega) \int_{-\infty}^{\infty} \frac{d \varepsilon}{2 \pi} \operatorname{Sp}\left[\underline{\sigma}_{1} \underline{G}^{0}(\varepsilon+\omega) \underline{G}^{0}(\varepsilon)\right] .
$$

Correspondingly the $\mathrm{n}$-th order contribution is

$$
\begin{array}{r}
g^{(n)}(\omega)=\frac{1}{2 i} \int_{-\infty}^{\infty} \frac{d \omega_{1} \cdots d \omega_{n-1}}{(2 \pi)^{n-1}}\left(V\left(\omega_{1}\right) \cdots V\left(\omega_{n-1}\right) V\left(\omega-\left(\omega_{1}+\cdots+\omega_{n-1}\right)\right) \times\right. \\
\left.\int_{-\infty}^{\infty} \frac{d \varepsilon}{2 \pi} \operatorname{Sp}\left[\underline{\sigma}_{1} \underline{G}^{0}(\varepsilon+\omega) \underline{G}^{0}\left(\varepsilon+\omega_{1}+\cdots+\omega_{n-1}\right) \cdots \underline{G}^{0}\left(\varepsilon+\omega_{1}\right) \underline{G}^{0}(\varepsilon)\right]\right) .
\end{array}
$$

By rewriting this expression as

$$
\begin{array}{r}
g^{(n)}(\omega)=: \int_{-\infty}^{\infty} \frac{d \omega_{1} \cdots d \omega_{n-1}}{(2 \pi)^{n-1}}\left[V\left(\omega_{1}\right) \cdots V\left(\omega_{n-1}\right) V\left(\omega-\left(\omega_{1}+\cdots+\omega_{n-1}\right)\right) \times\right. \\
\left.\mathcal{K}^{(n)}\left(\omega_{1}, \cdots, \omega_{n-1}, \omega-\left(\omega_{1}+\cdots+\omega_{n-1}\right)\right)\right],
\end{array}
$$

one introduces the response function

$$
\begin{array}{r}
\mathcal{K}^{(n)}\left(\omega_{1}, \cdots, \omega_{n-1}, \omega_{n}\right):=\frac{1}{2 i} \int_{-\infty}^{\infty} \frac{d \varepsilon}{2 \pi} \operatorname{Sp}\left[\underline{\sigma}_{1} \underline{G}^{0}\left(\varepsilon+\omega_{1}+\cdots+\omega_{n}\right) \times\right. \\
\left.\underline{G}^{0}\left(\varepsilon+\omega_{1}+\cdots+\omega_{n-1}\right) \cdots \underline{G}^{0}\left(\varepsilon+\omega_{1}\right) \underline{G}^{0}(\varepsilon)\right] .
\end{array}
$$

To finally express the response function through retarded and advanced functions one has to evaluate the matrix-products and use Eq.(A.10). It is convenient to use the abbreviations

$$
\underline{G}_{0}:=\underline{G}^{0}(\varepsilon) ; \underline{G}_{i}:=\underline{G}^{0}\left(\varepsilon+\cdots+\omega_{i}\right)
$$

and

$$
h_{0}:=\tanh \left(\frac{\varepsilon \beta}{2}\right) ; h_{i}:=\tanh \left(\frac{\left(\varepsilon+\cdots+\omega_{i}\right) \beta}{2}\right) .
$$

Consider the matrix-product of three Green's functions:

$$
\begin{aligned}
& \underline{G}_{2} \underline{G}_{1} \underline{G}_{0}=\left(\begin{array}{cc}
\mathcal{G}_{2}^{R} \mathcal{G}_{1}^{R} & \mathcal{G}_{2}^{R} \mathcal{G}_{1}^{K}+\mathcal{G}_{2}^{K} \mathcal{G}_{1}^{A} \\
0 & \mathcal{G}_{2}^{A} \mathcal{G}_{1}^{A}
\end{array}\right)\left(\begin{array}{cc}
\mathcal{G}_{0}^{R} & \mathcal{G}_{0}^{K} \\
0 & \mathcal{G}_{0}^{A}
\end{array}\right) \\
& =\left(\begin{array}{cc}
\mathcal{G}_{2}^{R} \mathcal{G}_{1}^{R} \mathcal{G}_{0}^{R} & \mathcal{G}_{2}^{R} \mathcal{G}_{1}^{R} \mathcal{G}_{0}^{K}+\left[\mathcal{G}_{2}^{R} \mathcal{G}_{1}^{K}+\mathcal{G}_{2}^{K} \mathcal{G}_{1}^{A}\right] \mathcal{G}_{0}^{A} \\
0 & \mathcal{G}_{2}^{A} \mathcal{G}_{1}^{A} \mathcal{G}_{0}^{A}
\end{array}\right) .
\end{aligned}
$$


The generalization to products of $n$ matrices $\underline{G}_{n} \cdots \underline{G}_{0}$ is straightforward. The Keldysh component is given by the sum of all different products with just one factor $\mathcal{G}_{i}^{K}$ where there are only retarded functions left of $\mathcal{G}_{i}^{K}$ and only advanced right of it. For the $n$-th order response function one obtains

$$
\begin{gathered}
\mathcal{K}^{(n)}\left(\omega_{1}, \cdots, \omega_{n-1}, \omega_{n}\right)=\frac{1}{2 i} \int_{-\infty}^{\infty} \frac{d \varepsilon}{2 \pi}\left(h_{0} \mathcal{G}_{n}^{R} \cdots \mathcal{G}_{1}^{R}\left[\mathcal{G}_{0}^{R}-\mathcal{G}_{0}^{A}\right]+\right. \\
\left.h_{1} \mathcal{G}_{n}^{R} \cdots \mathcal{G}_{2}^{R}\left[\mathcal{G}_{1}^{R}-\mathcal{G}_{1}^{A}\right] \mathcal{G}_{0}^{A}+\cdots+h_{n}\left[\mathcal{G}_{n}^{R}-\mathcal{G}_{n}^{A}\right] \mathcal{G}_{n-1}^{A} \cdots \mathcal{G}_{0}^{A}\right)
\end{gathered}
$$

It will now be demonstrated, how this response function can be obtained within the imaginary-time Matsubara technique.

\section{A.2 The Matsubara response function}

The Matsubara Green's function is defined as

$$
\mathcal{G}_{\mathbf{k}, \mathbf{k}^{\prime}}\left(\tau, \tau^{\prime}\right):=-\left\langle\hat{T} \hat{\Psi}_{H, \mathbf{k}}(\tau) \hat{\Psi}_{H, \mathbf{k}^{\prime}}^{\dagger}\left(\tau^{\prime}\right)\right\rangle
$$

where $\hat{T}$ is the time-ordering-operator, and for equal times operators are ordered so, that generators stand left of annihilators. The Matsubara function itself is defined only for systems in equilibrium and thus only depends on the (imaginary-) time-difference $\tau-\tau^{\prime}$. Here it will for the moment be treated formally like a nonequilibrium quantity analogous to the Keldysh function. The following steps are purely formal and can not be given any direct physical meaning. First the examples given in Eqs.(A.3), A.4) are reconsidered and analogous expressions in imaginary time are written down.

$$
\begin{gathered}
\mathbf{j}_{\text {para }}(\mathbf{k}, \tau)=-\frac{e}{2 m} \sum_{\mathbf{q}}(\mathbf{k}+2 \mathbf{q})\left\langle\hat{\Psi}_{H, \mathbf{k}+\mathbf{q}}^{\dagger}(\tau) \hat{\Psi}_{H, \mathbf{q}}(\tau)\right\rangle \\
=-\frac{e}{2 m} \sum_{\mathbf{q}}(\mathbf{k}+2 \mathbf{q}) \mathcal{G}_{\mathbf{q}, \mathbf{k}+\mathbf{q}}(\tau, \tau) \\
n(\mathbf{k}, \tau)=\sum_{\mathbf{q}}\left\langle\hat{\Psi}_{H, \mathbf{k}+\mathbf{q}}^{\dagger}(\tau) \hat{\Psi}_{H, \mathbf{q}}(\tau)\right\rangle=\sum_{\mathbf{q}} \mathcal{G}_{\mathbf{q}, \mathbf{k}+\mathbf{q}}(\tau, \tau) .
\end{gathered}
$$

Now an auxiliary quantity $\tilde{V}_{\mathbf{q}, \mathbf{q}^{\prime}}(\tau)$ which fulfills Bosonic Matsubara boundaryconditions is introduced:

$$
\tilde{V}_{\mathbf{q}, \mathbf{q}^{\prime}}(\tau-n \beta)=\tilde{V}_{\mathbf{q}, \mathbf{q}^{\prime}}(\tau)
$$


for any integer number $n$. Even though this quantity is not the analytic continuation of any physical potential, it will formally be treated like an external field and one defines the operator

$$
\hat{H}_{1}(\tau):=\sum_{\mathbf{q}, \mathbf{q}^{\prime}} \tilde{V}_{\mathbf{q}, \mathbf{q}^{\prime}}(\tau) \hat{\Psi}_{\mathbf{q}}^{\dagger} \hat{\Psi}_{\mathbf{q}^{\prime}}
$$

The time-evolution operator is also defined similar to the equilibrium situation,

$$
\hat{U}(\beta, 0)=\hat{T} \exp \left[-\int_{0}^{\beta} d \tau \hat{H}_{1, D}(\tau)\right],
$$

and the index $D$ stands for Dirac-picture:

$$
\hat{H}_{1, D}(\tau)=e^{\hat{H}_{0} \tau} \hat{H}_{1}(\tau) e^{-\hat{H}_{0} \tau} .
$$

The Green's function can be evaluated using the usual Feynman rules for the Matsubara technique:

$$
\mathcal{G}_{\mathbf{k}, \mathbf{k}^{\prime}}\left(\tau, \tau^{\prime}\right)=-\left\langle\hat{T} \hat{U}(\beta, 0) \hat{\Psi}_{D, \mathbf{k}}(\tau) \hat{\Psi}_{D, \mathbf{k}^{\prime}}^{\dagger}\left(\tau^{\prime}\right)\right\rangle_{0}^{c o n}
$$

Now the expectation value is calculated with respect to $\hat{H}_{0}$, as indicated by the index 0 , and con means, that only connected diagrams are taken into account. To describe the response of the system, analogous to the Keldysh situation the quantities

$$
\tilde{g}_{\mathbf{k}, \mathbf{k}^{\prime}}(\tau)=\mathcal{G}_{\mathbf{k}, \mathbf{k}^{\prime}}(\tau, \tau)
$$

and

$$
\tilde{g}_{\mathbf{k}, \mathbf{k}^{\prime}}(i \tilde{\omega})=\int_{0}^{\beta} d \tau e^{i \tilde{\omega} \tau} \tilde{g}_{\mathbf{k}, \mathbf{k}^{\prime}}(\tau)
$$

are defined. Here $\tilde{\omega}=(2 \pi n) / \beta$ are Bosonic Matsubara frequencies. In the following Bosonic frequencies, in contrast to Fermionic ones, will always be marked by a 'tilde'. Momentum-variables will now again be suppressed and summation over internal momenta will be assumed implicitly. The diagrams contributing to $\tilde{g}(i \tilde{\omega})$ are equal to thous shown in Fig.A.1 but with the real frequencies replaced by the corresponding Matsubara frequencies. The $n$-th order contribution is given by

$$
\begin{aligned}
& \tilde{g}^{(n)}(i \tilde{\omega})= \frac{1}{(\beta)^{n-1}} \sum_{\tilde{\omega}_{1} \cdots \tilde{\omega}_{n-1}} \tilde{V}\left(i \tilde{\omega}_{1}\right) \cdots \tilde{V}\left(i \tilde{\omega}_{n-1}\right) \tilde{V}\left(i \tilde{\omega}-i\left(\tilde{\omega}_{1}+\cdots+\tilde{\omega}_{n-1}\right)\right) \times \\
& \frac{1}{\beta} \sum_{\varepsilon} \mathcal{G}^{0}(i \varepsilon) \mathcal{G}^{0}\left(i \varepsilon+i \tilde{\omega}_{1}\right) \cdots \mathcal{G}^{0}\left(i \varepsilon+\cdots+i \tilde{\omega}_{n-1}\right) \mathcal{G}^{0}(i \varepsilon+i \tilde{\omega}) \\
&= \frac{1}{(\beta)^{n-1}} \sum_{\tilde{\omega}_{1} \cdots \tilde{\omega}_{n-1}} \tilde{V}\left(i \tilde{\omega}_{1}\right) \cdots \tilde{V}\left(i \tilde{\omega}_{n-1}\right) \tilde{V}\left(i \tilde{\omega}-i\left(\tilde{\omega}_{1}+\cdots+\tilde{\omega}_{n-1}\right)\right) \times \\
& \tilde{\mathcal{K}}^{(n)}\left(i \tilde{\omega}_{1}, \cdots, i \tilde{\omega}_{n-1}, i \tilde{\omega}-i\left(\tilde{\omega}_{1}+\cdots+\tilde{\omega}_{n-1}\right)\right),
\end{aligned}
$$


and the Matsubara response function is defined as

$$
\begin{array}{r}
\tilde{\mathcal{K}}^{(n)}\left(i \tilde{\omega}_{1}, \cdots, i \tilde{\omega}_{n-1}, i \tilde{\omega}_{n}\right):=\frac{1}{\beta} \sum_{\varepsilon} \mathcal{G}^{0}(i \varepsilon) \mathcal{G}^{0}\left(i \varepsilon+i \tilde{\omega}_{1}\right) \times \\
\ldots \mathcal{G}^{0}\left(i \varepsilon+\cdots+i \tilde{\omega}_{n-1}\right) \mathcal{G}^{0}\left(i \varepsilon+i \tilde{\omega}_{1}+\cdots+i \tilde{\omega}_{n}\right) .
\end{array}
$$

For positive frequencies $\omega>0$ the equilibrium Matsubara function is equal to the analytic continuation of the retarded function, and for $\omega<0$ to the continuation of the advanced function:

$$
\begin{array}{ll}
\mathcal{G}^{0}(i \omega)=\mathcal{G}^{0, R}(i \omega) & \text { for } \omega>0 \\
\mathcal{G}^{0}(i \omega)=\mathcal{G}^{0, A}(i \omega) & \text { for } \omega<0 .
\end{array}
$$

With the abbreviations

$$
\tilde{\mathcal{G}}_{0}^{R / A}(z):=\mathcal{G}^{0, R / A}(z) ; \tilde{\mathcal{G}}_{i}^{R / A}(z):=\mathcal{G}^{0, R / A}\left(z+i \tilde{\omega}_{1} \cdots+i \tilde{\omega}_{i}\right)
$$

and the choice $\tilde{\omega}_{i}>0$ Eq. A.34) can be written as

$$
\begin{array}{r}
\tilde{\mathcal{K}}^{(n)}\left(i \tilde{\omega}_{1}, \cdots, i \tilde{\omega}_{n}\right)=\frac{1}{\beta}\left[\sum_{\varepsilon>0} \tilde{\mathcal{G}}_{0}^{R}(i \varepsilon) \cdots \tilde{\mathcal{G}}_{n}^{R}(i \varepsilon)+\sum_{0>\varepsilon>-\tilde{\omega}_{1}} \tilde{\mathcal{G}}_{0}^{A}(i \varepsilon) \tilde{\mathcal{G}}_{1}^{R}(i \varepsilon) \cdots \tilde{\mathcal{G}}_{n}^{R}(i \varepsilon)+\right. \\
\left.\sum_{-\tilde{\omega}_{1}>\varepsilon>-\left(\tilde{\omega}_{1}+\tilde{\omega}_{2}\right)} \tilde{\mathcal{G}}_{0}^{A}(i \varepsilon) \tilde{\mathcal{G}}_{1}^{A}(i \varepsilon) \tilde{\mathcal{G}}_{2}^{R}(i \varepsilon) \cdots \tilde{\mathcal{G}}_{n}^{R}(i \varepsilon)+\cdots+\sum_{-\left(\tilde{\omega}_{1}+\cdots+\tilde{\omega}_{n}\right)>\varepsilon} \tilde{\mathcal{G}}_{0}^{A}(i \varepsilon) \cdots \tilde{\mathcal{G}}_{n}^{A}(i \varepsilon)\right] .
\end{array}
$$

The frequency sums are evaluated by contour-integration:

$$
\frac{1}{\beta} \sum_{\varepsilon>0} \tilde{\mathcal{G}}_{0}^{R}(i \varepsilon) \cdots \tilde{\mathcal{G}}_{n}^{R}(i \varepsilon)=-\frac{1}{2 \pi i} \int_{-\infty}^{\infty} d \varepsilon f(\varepsilon) \tilde{\mathcal{G}}_{0}^{R}(\varepsilon) \cdots \tilde{\mathcal{G}}_{n}^{R}(\varepsilon)
$$

and

$$
\begin{gathered}
\frac{1}{\beta} \sum_{0>\varepsilon>-\tilde{\omega}_{1}} \tilde{\mathcal{G}}_{0}^{A}(i \varepsilon) \tilde{\mathcal{G}}_{1}^{R}(i \varepsilon) \cdots \tilde{\mathcal{G}}_{n}^{R}(i \varepsilon)=-\frac{1}{2 \pi i}\left[-\int_{-\infty}^{\infty} d \varepsilon f(\varepsilon) \tilde{\mathcal{G}}_{0}^{A}(\varepsilon) \tilde{\mathcal{G}}_{1}^{R}(\varepsilon) \cdots \tilde{\mathcal{G}}_{n}^{R}(\varepsilon)\right. \\
\left.\quad+\int_{-\infty}^{\infty} d \varepsilon f(\varepsilon) \tilde{\mathcal{G}}_{0}^{A}\left(\varepsilon-i \tilde{\omega}_{1}\right) \tilde{\mathcal{G}}_{1}^{R}\left(\varepsilon-i \tilde{\omega}_{1}\right) \cdots \tilde{\mathcal{G}}_{n}^{R}\left(\varepsilon-i \tilde{\omega}_{1}\right)\right]
\end{gathered}
$$

$f(\varepsilon)=1 /(\exp (\beta \varepsilon)+1)$ is the Fermi function. The analytic continuation to the real axis according to $i \tilde{\omega}_{n} \rightarrow \omega_{n}+i 0$ results in

$$
\begin{aligned}
& \frac{1}{\beta} \sum_{\varepsilon>0} \tilde{\mathcal{G}}_{0}^{R}(i \varepsilon) \cdots \tilde{\mathcal{G}}_{n}^{R}(i \varepsilon)+\frac{1}{\beta} \sum_{0>\varepsilon>-\tilde{\omega}_{1}} \tilde{\mathcal{G}}_{0}^{A}(i \varepsilon) \tilde{\mathcal{G}}_{1}^{R}(i \varepsilon) \cdots \tilde{\mathcal{G}}_{n}^{R}(i \varepsilon) \\
& \rightarrow-\frac{1}{2 \pi i} \int_{-\infty}^{\infty} d \varepsilon\left[f(\varepsilon)\left[\mathcal{G}_{0}^{R}-\mathcal{G}_{0}^{A}\right] \mathcal{G}_{1}^{R} \cdots \mathcal{G}_{n}^{R}+f\left(\varepsilon+\omega_{1}\right) \mathcal{G}_{0}^{A} \mathcal{G}_{1}^{R} \cdots \mathcal{G}_{n}^{R}\right],
\end{aligned}
$$


with the abbreviations defined in Eq.(A.19). Similarly all remaining terms are evaluated and one obtains the analytically continued response function

$$
\begin{array}{r}
\tilde{\mathcal{K}}^{(n)}\left(\omega_{1}, \cdots, \omega_{n}\right)=-\frac{1}{i} \int_{-\infty}^{\infty} \frac{d \varepsilon}{2 \pi}\left[f(\varepsilon)\left[\mathcal{G}_{0}^{R}-\mathcal{G}_{0}^{A}\right] \mathcal{G}_{1}^{R} \cdots \mathcal{G}_{n}^{R}+f\left(\varepsilon+\omega_{1}\right) \mathcal{G}_{0}^{A}\left[\mathcal{G}_{1}^{R}-\right.\right. \\
\left.\left.\mathcal{G}_{1}^{A}\right] \mathcal{G}_{2}^{R} \cdots \mathcal{G}_{n}^{R}+\cdots+f\left(\varepsilon+\omega_{1}+\cdots+\omega_{n}\right) \mathcal{G}_{0}^{A} \cdots \mathcal{G}_{n-1}^{A}\left[\mathcal{G}_{n}^{R}-\mathcal{G}_{n}^{A}\right]\right]
\end{array}
$$

Now $f(\varepsilon)$ is connected to $h(\varepsilon):=\tanh (\varepsilon \beta / 2)$ via

$$
f(\varepsilon)=\frac{1-h(\varepsilon)}{2}
$$

and since integrals over products of only retarded or only advanced Green's functions vanish, one finally arrives at

$$
\begin{aligned}
\tilde{\mathcal{K}}^{(n)}\left(\omega_{1}, \cdots, \omega_{n}\right) & =\frac{1}{2 i} \int_{-\infty}^{\infty} \frac{d \varepsilon}{2 \pi}\left[h_{1}\left[\mathcal{G}_{0}^{R}-\mathcal{G}_{0}^{A}\right] \mathcal{G}_{1}^{R} \cdots \mathcal{G}_{n}^{R}+h_{2} \mathcal{G}_{0}^{A}\left[\mathcal{G}_{1}^{R}-\mathcal{G}_{1}^{A}\right] \mathcal{G}_{2}^{R} \cdots \mathcal{G}_{n}^{R}\right. \\
& \left.+\cdots+h_{n} \mathcal{G}_{0}^{A} \cdots \mathcal{G}_{n-1}^{A}\left[\mathcal{G}_{n}^{R}-\mathcal{G}_{n}^{A}\right]\right]
\end{aligned}
$$

This is just the same result as obtained within the real-time Keldysh technique in Eq.(A.22). 


\section{Appendix B}

\section{Fluctuation-dissipation theorem}

In Chap.2.3 the differential equation for the Cooperon in a time-dependent external field $V(\mathbf{r}, t)$ was derived. This equation is used in Chap. 3.3 to obtain the dephasing time due to Nyquist noise. In this case it is assumed, that the fluctuating field is generated by the diffusive motion of the background electrons. Since the electrons are neither 'external' (i.e. they have to satisfy the Pauli principle) nor classical, this procedure is quite subtle and part of the origin for the recent controversy about zero temperature dephasing [38, 32, 43, 25]. To be free of any ambiguities and physically motivated cutoffs one must in principle treat electron-electron interactions quantum mechanically from the beginning. The derivation of an equation for the Cooperon in the presence of quantum fields is still an open problem. In the following the steps leading to the semiclassical Nyquist noise result will be outlined. Since the whole system is in thermodynamic equilibrium, the total electric scalar potential generated by the Coulomb charge of the electrons must be time independent. After averaging over disorder translational invariance is restored and thus due to charge neutrality the average scalar potential cancels with the potential $V_{a t}$ from the fixed positive background charge of the atoms in the system円. Quantum mechanically the scalar potential generated by the electrons is described by the observable

$$
\hat{V}(\mathbf{r}, t)=\int d \mathbf{r}^{\prime} f\left(\mathbf{r}^{\prime}-\mathbf{r}\right) \hat{n}_{H}\left(\mathbf{r}^{\prime}, t\right) .
$$

$\hat{n}_{H}\left(\mathbf{r}^{\prime}, t\right)=\hat{\Psi}_{H}^{\dagger}\left(\mathbf{r}^{\prime}, t\right) \hat{\Psi}_{H}\left(\mathbf{r}^{\prime}, t\right)$ is the density-operator in the Heisenberg representation. The disorder average of the quantum-statistical expectation value $\langle\cdots\rangle=$ $\mathrm{Sp}\left[e^{-\beta \hat{H}} \ldots\right] / \mathrm{Sp}\left[e^{-\beta \hat{H}}\right]$ is thus given by $\overline{\langle\hat{V}(\mathbf{r}, t)\rangle}=-V_{a t}$. In momentum-frequency space one obtains

$$
\hat{V}_{\mathbf{p}}(\omega)=\int d \mathbf{r} d t \hat{V}(\mathbf{r}, t) e^{-i(\mathbf{p} \cdot \mathbf{r}-\omega t)}=f_{\mathbf{p}} \hat{n}_{\mathbf{p}}(\omega),
$$

\footnotetext{
${ }^{1}$ Here a jellium model is presumed where the positive background charge is uniformly distributed in space.
} 
where $f_{\mathbf{p}}$ is for different dimensions given in Eqs.(2.79) - (2.81) and $\hat{n}_{\mathbf{p}}=$ $\int d \mathbf{r} e^{-i \mathbf{p} \cdot \mathbf{r}} \hat{n}(\mathbf{r})$ is the Fourier transform of $\hat{n}(\mathbf{r})$. Assuming a Gaussian probability distribution, the Fourier transform of the random Nyquist noise field $V(\mathbf{p}, \omega)$ will now be defined through its first and second moment. The first moment vanishes and the second moment is defined as the diffusive limit $\left(|\mathbf{p}|<1 / l\right.$ and $\left.|\omega|<1 / \tau_{e l}\right)$ of the averaged correlator for $\hat{V}_{\mathbf{p}}(\omega)$. Since the correlation function is an observable quantity, it must be expressed through an hermitian operator. The canonical way to do this, is to use the symmetric product [76] $(1 / 2)\left[\hat{V}_{\mathbf{p}}(\omega) \hat{V}_{\mathbf{p}^{\prime}}\left(\omega^{\prime}\right)+\hat{V}_{\mathbf{p}^{\prime}}\left(\omega^{\prime}\right) \hat{V}_{\mathbf{p}}(\omega)\right]$ which meets the hermiticity condition. Consequently one defines $V(\mathbf{p}, \omega)$ and the Nyquist noise probability distribution via

$$
\begin{aligned}
\langle V(\mathbf{p}, \omega)\rangle_{n n} & :=0 \\
\left\langle V(\mathbf{p}, \omega) V\left(\mathbf{p}^{\prime}, \omega^{\prime}\right)\right\rangle_{n n} & :=\frac{1}{2} \overline{\left\langle\hat{V}_{\mathbf{p}}(\omega) \hat{V}_{\mathbf{p}^{\prime}}\left(\omega^{\prime}\right)+\hat{V}_{\mathbf{p}^{\prime}}\left(\omega^{\prime}\right) \hat{V}_{\mathbf{p}}(\omega)\right\rangle}-\overline{\left\langle V_{\mathbf{p}}(\omega)\right\rangle} \frac{}{\left\langle V_{\mathbf{p}^{\prime}}\left(\omega^{\prime}\right)\right\rangle},
\end{aligned}
$$

where momenta and frequencies are limited to $|\mathbf{p}|,\left|\mathbf{p}^{\prime}\right|<1 / l$ and $|\omega|,\left|\omega^{\prime}\right|<1 / \tau_{e l}$. Averaging over the Nyquist noise distribution is denoted by $\langle\cdots\rangle_{n n}$. From translational invariance in space and time it follows, that

$$
\frac{1}{2} \overline{\left\langle\hat{V}_{\mathbf{p}}(\omega) \hat{V}_{\mathbf{p}^{\prime}}\left(\omega^{\prime}\right)+\hat{V}_{\mathbf{p}^{\prime}}\left(\omega^{\prime}\right) \hat{V}_{\mathbf{p}}(\omega)\right\rangle}=\mathcal{V} \delta_{\mathbf{p}, \mathbf{p}^{\prime}} 2 \pi \delta\left(\omega-\omega^{\prime}\right) f_{\mathbf{p}}^{2} \frac{i}{2} \overline{\mathcal{P}^{K}}(\mathbf{p}, \omega)
$$

where the Keldysh component of the two-particle Green's function $\mathcal{P}^{K}$ is introduced in analogy to the one-particle function $\mathcal{G}^{K}$ in Eq.(A.6),

$$
\mathcal{P}^{K}(\mathbf{p}, \omega):=-i \int d \mathbf{r} d t e^{-i(\mathbf{p} \cdot \mathbf{r}-\omega t)}\langle\hat{n}(\mathbf{r}, t) \hat{n}(0,0)+\hat{n}(0,0) \hat{n}(\mathbf{r}, t)\rangle .
$$

The retarded and advanced functions are defined as

$$
\begin{aligned}
& \mathcal{P}^{R}(\mathbf{p}, \omega):=-i \int d \mathbf{r} d t e^{-i(\mathbf{p} \cdot \mathbf{r}-\omega t)} \Theta(t)\langle\hat{n}(\mathbf{r}, t) \hat{n}(0,0)-\hat{n}(0,0) \hat{n}(\mathbf{r}, t)\rangle, \\
& \mathcal{P}^{A}(\mathbf{p}, \omega):=i \int d \mathbf{r} d t e^{-i(\mathbf{p} \cdot \mathbf{r}-\omega t)} \Theta(-t)\langle\hat{n}(\mathbf{r}, t) \hat{n}(0,0)-\hat{n}(0,0) \hat{n}(\mathbf{r}, t)\rangle .
\end{aligned}
$$

For the single-particle functions the relation between the different components is given by Eq.(A.10). The corresponding connection in the two-particle case is

$$
\mathcal{P}^{K}(\mathbf{p}, \omega)=\operatorname{coth}\left(\frac{\omega \beta}{2}\right)\left[\mathcal{P}^{R}(\mathbf{p}, \omega)-\mathcal{P}^{A}(\mathbf{p}, \omega)\right]=2 i \operatorname{coth}\left(\frac{\omega \beta}{2}\right) \operatorname{Im} \mathcal{P}^{R}(\mathbf{p}, \omega) .
$$

Inserting this in Eq.(B.5) yields

$$
\frac{1}{2} \overline{\left\langle\hat{V}_{\mathbf{p}}(\omega) \hat{V}_{\mathbf{p}^{\prime}}\left(\omega^{\prime}\right)+\hat{V}_{\mathbf{p}^{\prime}}\left(\omega^{\prime}\right) \hat{V}_{\mathbf{p}}(\omega)\right\rangle}=-\mathcal{V} \delta_{\mathbf{p}, \mathbf{p}^{\prime}} 2 \pi \delta\left(\omega-\omega^{\prime}\right) f_{\mathbf{p}}^{2} \operatorname{coth}\left(\frac{\omega \beta}{2}\right) \operatorname{Im} \overline{\mathcal{P}^{R}}(\mathbf{p}, \omega) .
$$


The retarded Green's function $\mathcal{P}^{R}$ can be obtained from the Matsubara function

$$
\mathcal{P}(\mathbf{p}, i \tilde{\omega})=-\int_{0}^{\beta} d \tau \int d \mathbf{r} e^{-i(\mathbf{p} \cdot \mathbf{r}-\tilde{\omega} \tau)}\langle\hat{T} \hat{n}(\mathbf{r}, \tau) \hat{n}(0,0)\rangle
$$

by the usual continuation $i \tilde{\omega} \rightarrow \omega+i 0$. Using Wick's theorem, the expectation value can be decomposed in totally connected diagrams and a product of the average particle numbers,

$$
\langle\hat{T} \hat{n}(\mathbf{r}, \tau) \hat{n}(0,0)\rangle=\langle\hat{T} \hat{n}(\mathbf{r}, \tau) \hat{n}(0,0)\rangle^{c o n}+\langle\hat{T} \hat{n}(\mathbf{r}, \tau)\rangle\langle\hat{T} \hat{n}(0,0)\rangle .
$$

Only the connected diagrams contribute to the Nyquist noise correlator defined in Eq.(B.4) since the second term is canceled by the subtracted averages in Eq.(B.4). But the connected diagrams just yield the usual polarization $\Pi(\mathbf{p}, i \tilde{\omega})$ of the system,

$$
\Pi(\mathbf{p}, i \tilde{\omega})=-\int_{0}^{\beta} d \tau \int d \mathbf{r} e^{-i(\mathbf{p} \cdot \mathbf{r}-\tilde{\omega} \tau)}\langle\hat{T} \hat{n}(\mathbf{r}, \tau) \hat{n}(0,0)\rangle^{c o n}
$$

Looking at Eq. (B.10) one thus has to evaluate the function

$$
f_{\mathbf{p}}^{*}(i \tilde{\omega}):=\operatorname{Im} f_{\mathbf{p}} \bar{\Pi}(\mathbf{p}, i \tilde{\omega}) f_{\mathbf{p}}=\operatorname{Im}\left[f_{\mathbf{q}}+f_{\mathbf{p}} \bar{\Pi}(\mathbf{p}, i \tilde{\omega}) f_{\mathbf{p}}\right]
$$

Diagrammatically $f_{\mathbf{p}}^{*}(i \tilde{\omega})$ is depicted in Fig.B.1. The total polarization $\Pi$ can be

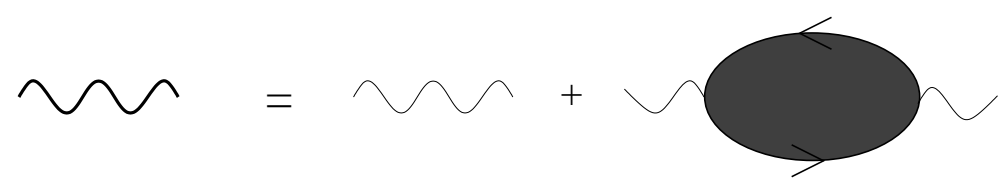

Figure B.1: Diagrammatic expression for the effective interaction. The thick wiggled line represents $f^{\mathrm{RPA}}(\mathbf{q}, \omega)$ while thin wiggled ones stand for the bare interaction $f_{\mathbf{q}}$. The shaded bubble is the disorder averaged total polarization $\bar{\Pi}(\mathbf{p}, i \tilde{\omega})$.

decomposed into a series of diagrams containing only the irreducible polarization $\Pi_{0}$ as shown in Fig.2.6 (a) for diffusive systems. Obviously the function $f^{*}$ is nothing but the imaginary part of the effective RPA-interaction $f^{\mathrm{RPA}}(\mathbf{p}, \omega)=f_{\mathbf{q}} / \epsilon(\mathbf{q}, \omega)$ and from Eq.(B.10) together with the definition (B.4) one obtains

$$
\left\langle V(\mathbf{p}, \omega) V\left(\mathbf{p}^{\prime}, \omega^{\prime}\right)\right\rangle_{n n}=-\mathcal{V} \delta_{\mathbf{p}, \mathbf{p}^{\prime}} 2 \pi \delta\left(\omega-\omega^{\prime}\right) \operatorname{coth}\left(\frac{\omega \beta}{2}\right) \operatorname{Im} f^{\mathrm{RPA}}(\mathbf{p}, \omega) .
$$

Using the approximation for the classical diffusive limit with $|\mathbf{p}|<1 / l$ and $|\omega|<$ $1 / \tau_{e l}$ given in Eq.(2.95), finally yields

$$
\left\langle V(\mathbf{p}, \omega) V\left(\mathbf{p}^{\prime}, \omega^{\prime}\right)\right\rangle_{n n}=\mathcal{V} \delta_{\mathbf{p}, \mathbf{p}^{\prime}} 2 \pi \delta\left(\omega-\omega^{\prime}\right) \operatorname{coth}\left(\frac{\omega \beta}{2}\right) \frac{\omega}{\nu_{d} D \mathbf{p}^{2}} .
$$




\section{Bibliography}

[1] A. Völker and P. Kopietz, Dephasing in Disordered Conductors due to Fluctuating Electric Fields, cond-mat/9906320, to appear in Phys. Rev. B.

[2] P. Kopietz and A. Völker, Fermi's golden rule in a mesoscopic metal ring, Superlattices and Microstructures 26, 367 (1999).

[3] P. Kopietz and A. Völker, The weak localization correction to the polarization and persistent currents in mesoscopic metal rings, Phys. Lett. A 224, 569 (1998).

[4] W. Zwerger, Theory of Quantum Transport in Quantum Transport and Dissipation edited by T. Dittrich et al. (Wiley-VCH, Weinheim, 1998).

[5] S. Chakravarty and A. Schmid, Phys. Rep. 140, 193 (1986).

[6] For a recent review see Y. Imry, Introduction to Mesoscopic Physics, (Oxford University Press, New York, 1997).

[7] D. K. Ferry and S. M. Goodnick, Transport in Nanostructures, (Cambridge University Press, Cambridge, 1997).

[8] B. L. Altshuler, A. G. Aronov, and D. E. Khmelnitskii, J. Phys. C 15, 7367 (1982).

[9] A. Stern, Y. Aharonov, and Y. Imry, Phys. Rev. A 41, 3436 (1990).

[10] N. W. Ashcroft and N. D. Mermin, Solid State Physics, (Saunders College Publishing, Fort Worth, 1976).

[11] A.A.Abrikosov, L.P.Gorkov and I.E.Dzyaloshinski, Methods of Quantum Field Theorie in Statistical Physics (Dover, New York, 1975).

[12] J. Rammer and H. Smith, Rev. Mod. Phys. 58, 323 (1986).

[13] H.Fukuyama, Progress of Theo. Physics 84, 47 (1985).

[14] K.B.Efetov, Advances in Phys. 32, 53 (1983). 
[15] W. Eiler, J. Low Temp. Phys. 56, 481 (1984).

[16] A. Völker and P. Kopietz, Spectral correlations in disordered mesoscopic metals and their relevance for persistent currents, Mod. Phys. Lett. B. 10, 1397 (1996).

[17] S.Hikami, A.I. Larkin, and Y. Nagaoka, Porg. Theor. Phys. 63, 707 (1980).

[18] S. Wang and P. E. Lindelof, J. Low Temp. Phys. 71, 403 (1988).

[19] P. Mohanty, E.M.Q. Jariwala, and R.A. Webb, Phys. Rev. Lett. 78, 3366 (1997).

[20] B.L. Altshuler and A.G. Aronov in Electron-Electron Interactions in Disordered Systems, edited by A.L. Efros and M. Pollak (North Holland, Amsterdam, 1985).

[21] G.D.Mahan, Many-Particle Physics (Plenum Press, New York, 1990).

[22] B. L. Altshuler, A. G. Aronov, and D. E. Khmelnitskii, Solid State Commun. 39, 619 (1981).

[23] F. von Oppen and A. Stern, Phys. Rev. Lett. 79, 1114 (1997).

[24] D. G. Polyakov and K. V. Samokhin, Phys. Rev. Lett. 80, 1509 (1998).

[25] M. Vavilov and V. Ambegaokar, cond-mat/9902127.

[26] A. V. Svidzinskii, Zh. Eksp. Teor. Fiz. 31, 324 (1957) [Sov. Phys. JETP 4, 179 (1957)]; B. M. Barbashov, Zh. Eksp. Teor. Fiz. 48, 607 (1965) [Sov. Phys. JETP 21, 402 (1965)]; V. N. Popov, Functional Integrals in Quantum Field Theory and Statistical Physics, (Reidel, Dordrecht, 1983), Chap.4.

[27] E. S. Fradkin, Nucl. Phys. 76, 588 (1966).

[28] P. Kopietz, Int. J. Mod. Phys. B 10, 2111 (1996).

[29] H. Fukuyama and E. Abrahams, Phys. Rev. B 27, 5976 (1983).

[30] Y.M. Blanter, Phys.Rev. B 54, 12807 (1996).

[31] J. M. B. Lopes dos Santos, Phys. Rev. B 28, 1189 (1983).

[32] I. L. Aleiner, B. L. Altshuler, and M. E. Gershenson, Phys. Rev. Lett. 82, 3190 (1999); cond-mat/9808053.

[33] R. Raimondi, P. Schwab and C. Castellani, Phys. Rev. B 60, 5818 (1999).

[34] B. L. Altshuler, A. G. Aronov, D. E. Khmelnitskii, and A. I. Larkin in Quantum Theory of Solids, (Mir Publishers, Moscow, 1982). 
[35] P. Kopietz, Phys. Rev. Lett. 81, 2120 (1998).

[36] See, for example, P. Kopietz, Bosonization of Interacting Fermions in Arbitrary Dimensions, (Springer, Berlin, 1997).

[37] Y. Imry, H. Fukuyama, and P. Schwab, Europhys. Lett. 47, 608 (1999).

[38] D.S. Golubev and A.D. Zaikin, Phys. Rev. B 59, 9195 (1999).

[39] D.S. Golubev and A.D. Zaikin, cond-mat/9907494.

[40] U. Sivan, Y. Imry and A.G. Aronov, Europhys. Lett. 28, 115 (1994).

[41] See, for example, J. M. Ziman, Principles of the Theory of Solids, 2nd Edition, (Cambridge University Press, Cambridge, 1972).

[42] S. Wang and P. E. Lindelof, Phys. Rev. Lett. 59, 1156, (1987).

[43] B. L. Altshuler, M. E. Gershenson, and I. Aleiner, Physica E 3, 58 (1998).

[44] R. A. Webb, P. Mohanty, E. M. Q. Jariwala, T. R. Stevenson, and A. G. Zharikov, preprint (1998).

[45] A.B. Gougam, F. Pierre, H. Pothier, D. Esteve, and N.O. Birge, Proceedings of the LT-22 satellite conference "Electron Transport in Mesoscopic Systems", cond-mat/9912137.

[46] R. A. Webb et al., in Quantum Coherence and Decoherence, edited by K. Fujikawa and Y. A. Ono, (North Holland, 1998).

[47] D. G. Polyakov and K. V. Samokhin, Phys. Rev. Lett. 80, 1509 (1998).

[48] A.Lorke, R.J. Luyken, A.O. Govorov, J.P. Kotthaus, J.M. Garcia, and P.M. Petroff, Phys. Rev. Lett 84, 2223 (2000).

[49] F. Hund, Ann. Phys. (Leipzig) 32, 102 (1938); M. Büttiker, Y. Imry, and R. Landauer, Phys. Lett. 96 A, 365 (1983).

[50] L. P. Lévy, G. Dolan, J. Dunsmuir, and H. Bouchiat, Phys. Rev. Lett. 64, 2074 (1990).

[51] A. Kamenev, B. Reulet, H. Bouchiat, and Y. Gefen, Europhys. Lett. 28, 391 (1994).

[52] N. Trivedi and D.A. Browne, Phys. Rev. B 38, 9581 (1988).

[53] B. Reulet and H. Bouchiat, Phys. Rev. B 50, 2259 (1994). 
[54] J. Fricke and P. Kopietz, Phys. Rev. B 52, 2728 (1995); J. Fricke and P. Kopietz, cond-mat/9503078.

[55] P. Mohanty, E. M. Q. Jariwala, M. B. Ketchen, and R. A. Webb, in Quantum Coherence and Decoherence, edited by K. Fujikawa and Y. A. Ono (Elsevier, Amsterdam, 1996).

[56] V. E. Kravtsov and V. I. Yudson, Phys. Rev. Lett. 70, 210 (1993).

[57] A.G. Aronov, and V.E. Kravtsov, Phys. Rev. B 47, 13409 (1993).

[58] A. Schmid, Phys. Rev. Lett. 66, 80 (1991).

[59] A. L. Altshuler, Y. Gefen, and Y. Imry, Phys. Rev. Lett. 66, 88 (1991).

[60] V. I. Belinicher and B. I. Sturman, Usp. Fiz. Nauk 130, 130 (1980) [Sov. Phys. Usp. 23, 199 (1980)].

[61] V. I. Fal'ko and D. E. Khmel'nitskii, Zh. Eksp. Teor. Fiz. 95, 186 (1989) [Sov. Phys. JETP 68, 186 (1989)]; V. I. Fal'ko, Europhys. Lett. 8, 785 (1989).

[62] V. E. Kravtsov and V. I. Yudson, preprint cond-mat/9712149 (1997).

[63] G. Baym, Lectures on Quantum Mechanics, (Benjamin, New York, 1969).

[64] B.L.Altshuler und B.I.Shklovskii, Zh.Eksp.Teor.Fiz 91, 220 (1986).

[65] H.F. Cheung, K.Riedel und Y. Gefen, Phys. Rev. Lett 62, 587 (1989).

[66] V.E. Kravtsov and B.L. Altshuler, cond-mat/9910144.

[67] V. Ambegaokar and U. Eckern, Phys. Rev. Lett. 65, 381 (1990); ibid. 67, 3192 (1991).

[68] U. Eckern, Z. Phys. B 82, 393 (1991).

[69] P. Kopietz, Phys. Rev. Lett. 70, 3123 (1993); 71, 306 (1993) (E).

[70] A. Völker and P. Kopietz, Screening of persistent currents in mesoscopic metal rings, Z. Phys. B 102, 545 (1997).

[71] D. Vollhardt and P. Wölfle, Phys. Rev. B 22, 4666 (1980).

[72] M. T. Béal-Monod and G. Montambaux, Phys. Rev. B 46, 7182 (1992).

[73] K. B. Efetov, Phys. Rev. Lett. 76, 1908 (1996); Ya. M. Blanter and A. B. Mirlin, Phys. Rev. B 53, 12601 (1996); Phys. Rev. B 57, 4566 (1998).

[74] J.K.Cullum und R.A.Willoughby, Lanczos Algorithms for Large Symmetric Eigenvalue Computations Vol.I \& II (Birkhäuser, 1985). 
[75] A. Völker, Untersuchung von spektralen Korrelationen in ungeordneten mesoskopischen Metallen, Diploma Thesis (Universität Göttingen, 1996).

[76] Sh. Kogan, Electronic noise and fluctuations in solids, (Cambridge Univ. Press, Cambridge, 1996). 